\title{
ACTIONS OF MEASURED QUANTUM GROUPOIDS ON A FINITE BASIS
}

\author{
by
}

\author{
JONATHAN CRESPO
}

\begin{abstract}
In this article, we generalize to the case of measured quantum groupoids on a finite basis some important results concerning actions of locally compact quantum groups on $C^{*}$-algebras [5]. Let $\mathcal{G}$ be a measured quantum groupoid on a finite basis. We prove that if $\mathcal{G}$ is regular, then any weakly continuous action of $\mathcal{G}$ on a $\mathrm{C}^{*}$-algebra is necessarily strongly continuous. Following [3], we introduce and investigate a notion of $\mathcal{G}$-equivariant Hilbert $\mathrm{C}^{*}$-modules. By applying the previous results and a version of the Takesaki-Takai duality theorem obtained in [2] for actions of $\mathcal{G}$, we obtain a canonical equivariant Morita equivalence between a given $\mathcal{G}$ - $C^{*}$-algebra $A$ and the double crossed product $(A \rtimes \mathcal{G}) \rtimes \widehat{\mathcal{G}}$.
\end{abstract}

Keywords Locally compact quantum groups, measured quantum groupoids, monoidal equivalence, (semi-)regularity, Hilbert $C^{*}$-modules, Morita equivalence.

AMS classification 46L55, 16T99, 46L89.

\section{CONTENTS}

Introduction

1 Preliminary notations 4

2 Locally compact quantum groups 5

2.1 Hopf $C^{*}$-algebras associated with a quantum group . . . . . . . . . . . 6

2.2 Continuous actions of locally compact quantum groups . . . . . . . . . 7 7

2.3 Equivariant Hilbert $C^{*}$-modules and bimodules . . . . . . . . . . . . 7

3 Measured quantum groupoids 9

3.1 Case where the basis is finite-dimensional $\ldots \ldots \ldots \ldots$ 11. . . . . .

3.2 Weak Hopf $C^{*}$-algebras associated with a measured quantum groupoid on a

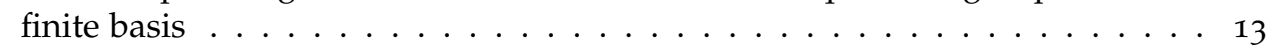

3.3 Measured quantum groupoid associated with a monoidal equivalence . . . . 14

4 Contributions to the notions of semi-regularity and regularity 17

5 Measured quantum groupoids on a finite basis in action 20

5.1 Continuous actions, crossed product and biduality . . . . . . . . . 20

5.2 Case of a colinking measured quantum groupoid . . . . . . . . . . . . 25

5.3 Actions of (semi-)regular measured quantum groupoids . . . . . . . . . . . 27

6 Notion of equivariant Hilbert $C^{*}$-modules 30

6.1 Actions of measured quantum groupoids on Hilbert $C^{*}$-modules . . . . . . 30

6.2 Case of a colinking measured quantum groupoid . . . . . . . . . . . . 40

6.3 Induction of equivariant Hilbert $C^{*}$-modules . . . . . . . . . . . . . . 44

7 Takesaki-Takai duality and equivariant Morita equivalence 54

8 Appendix 62

8.1 Normal linear forms, weights and operator-valued weights . . . . . . . 62

8.2 Relative tensor product and fiber product . . . . . . . . . . . . . . 64

8.3 Unitary equivalence of Hilbert $C^{*}$-modules $\ldots . . . \ldots \ldots$

References

Index of notations and symbols 
The notion of monoidal equivalence of compact quantum groups has been introduced by Bichon, De Rijdt and Vaes in [6]. Two compact quantum groups $G_{1}$ and $G_{2}$ are said to be monoidally equivalent if their categories of representations are equivalent as monoidal $C^{*}$-categories. They have proved that $\mathbb{G}_{1}$ and $\mathbb{G}_{2}$ are monoidally equivalent if and only if there exists a unital $C^{*}$-algebra equipped with commuting continuous ergodic actions of full multiplicity of $\mathbb{G}_{1}$ on the left and of $\mathbb{G}_{2}$ on the right.

Many crucial results of the geometric theory of free discrete quantum groups rely on the monoidal equivalence of their dual compact quantum groups. Among the applications of monoidal equivalence to this theory, we mention the contributions to randow walks and their associated boundaries [26, 15], CCAP property and Haagerup property [14], the Baum-Connes conjecture and K-amenability [29, 28].

In his Ph.D. thesis [12], De Commer has extended the notion of monoidal equivalence to the locally compact case. Two locally compact quantum groups $G_{1}$ and $G_{2}$ (in the sense of Kustermans and Vaes [19]) are said to be monoidally equivalent if there exists a von Neumann algebra equipped with a left Galois action of $G_{1}$ and a right Galois action of $G_{2}$ that commute. He proved that this notion is completely encoded by a measured quantum groupoid (in the sense of Enock and Lesieur [17]) on the basis $\mathbb{C}^{2}$. Such a groupoid is called a colinking measured quantum groupoid.

The measured quantum groupoids have been introduced and studied by Lesieur and Enock (see [17, 20]). Roughly speaking, a measured quantum groupoid (in the sense of Enock-Lesieur) is an octuple $\mathcal{G}=\left(N, M, \alpha, \beta, \Gamma, T, T^{\prime}, v\right)$, where $N$ and $M$ are von Neumann algebras (the basis $N$ and $M$ are the algebras of the groupoid corresponding respectively to the space of units and the total space for a classical groupoid), $\alpha$ and $\beta$ are faithful normal *-homomorphisms from $N$ and $N^{\circ}$ (the opposite algebra) to $M$ (corresponding to the source and target maps for a classical groupoid) with commuting ranges, $\Gamma$ is a coproduct taking its values in a certain fiber product, $v$ is a normal semi-finite weight on $N$ and $T$ and $T^{\prime}$ are operator-valued weights satisfying some axioms.

In the case of a finite-dimensional basis $N$, the definition has been greatly simplified by De Commer [11, 12] and we will use this point of view in this article. Broadly speaking, we can take for $v$ the non-normalized Markov trace on the $C^{*}$-algebra $N=\bigoplus_{1 \leqslant l \leqslant k} \mathrm{M}_{n_{l}}(\mathbb{C})$. The relative tensor product of Hilbert spaces (resp. the fiber product of von Neumann algebras) is replaced by the ordinary tensor product of Hilbert spaces (resp. von Neumann algebras). The coproduct takes its values in $M \otimes M$ but is no longer unital. In the following, these objects will be referred to as 'measured quantum groupoids on a finite basis'.

In [2], the authors introduce a notion of (strongly) continuous actions on $\mathrm{C}^{*}$-algebras of measured quantum groupoids on a finite basis. They extend the construction of the crossed product, the dual action and give a version of the Takesaki-Takai duality generalizing the Baaj-Skandalis duality theorem [3] in this setting.

If a colinking measured quantum groupoid $\mathcal{G}$, associated with a monoidal equivalence of two locally compact quantum groups $\mathbb{G}_{1}$ and $\mathbb{G}_{2}$, acts (strongly) continuously on a $C^{*}$-algebra $A$, then $A$ splits up as a direct sum $A=A_{1} \oplus A_{2}$ of $C^{*}$-algebras and the action of $\mathcal{G}$ on $A$ restricts to an action of $\mathbb{G}_{1}$ (resp. $G_{2}$ ) on $A_{1}$ (resp. $A_{2}$ ).

They also extend the induction procedure to the case of monoidally equivalent regular locally compact quantum groups. To any continuous action of $G_{1}$ on a $C^{*}$-algebra $A_{1}$, they associate canonically a $C^{*}$-algebra $A_{2}$ endowed with a continuous action of $\mathbb{G}_{2}$. As important consequences of this construction, we mention the following:

- a one-to-one functorial correspondence between the continuous actions of the quantum groups $G_{1}$ and $G_{2}$, which generalizes the compact case $[15]$ and the case of deformations by a 2-cocycle [21];

- a complete description of the continuous actions of colinking measured quantum groupoids;

- the equivalence of the categories $\mathrm{KK}_{\mathrm{G}_{1}}$ and $\mathrm{KK}_{\mathrm{G}_{2}}$, which generalizes to the regular locally compact case a result of Voigt [29]. 
The proofs of the above results rely crucially on the regularity of the quantum groups $G_{1}$ and $G_{2}$. They prove that the regularity of $G_{1}$ and $G_{2}$ is equivalent to the regularity in the sense of [16] (see also [23, 24]) of the associated colinking measured quantum groupoid. By passing, this result solves some questions raised in [21] in the case of deformations by a 2-cocycle.

In this article, we generalize to the case of (semi-)regular measured quantum groupoid on a finite basis some important properties of (semi-)regular locally compact quantum groups [4, 1]. This work will give us enough formulas to generalize some crucial results of [5] concerning actions of (semi-)regular locally compact quantum groups.

More precisely, if $\mathcal{G}$ is a semi-regular measured quantum groupoid on a finite basis, then the space consisting of the continuous elements of any action of $\mathcal{G}$ is a $C^{*}$-algebra. Moreover, if $\mathcal{G}$ is regular we prove that any weakly continuous action of $\mathcal{G}$ is necessarily continuous in the strong sense.

We introduce a notion of action of $\mathcal{G}$ on Hilbert $\mathrm{C}^{*}$-modules in line with the corresponding notion for quantum groups [3]. A $\mathcal{G}$-equivariant Hilbert $C^{*}$-module is a Hilbert $C^{*}$-module endowed with a continuous action (in a sense that will be specified). By using the previous result, if $\mathcal{G}$ is regular we prove that any action of $\mathcal{G}$ on a Hilbert $C^{*}$-module is necessarily continuous. We are able to define the notion of $\mathcal{G}$-equivariant Morita equivalence of $\mathcal{G}$ - $\mathrm{C}^{*}$ algebras. By applying a version of the Takesaki-Takai duality theorem obtained in [2], we prove that any $\mathcal{G}$-C ${ }^{*}$-algebra $A$ is $\mathcal{G}$-equivariantly Morita equivalent to its double crossed product $(A \rtimes \mathcal{G}) \rtimes \widehat{\mathcal{G}}$ in a canonical way.

This article is organized as follows.

- Chapter 1. We recall the general conventions and notations used throughout this paper.

- Chapter 2. We make an overview of the theory of locally compact quantum groups (cf. [19] and [4]). We recall the construction of the Hopf $C^{*}$-algebra associated with a locally compact quantum group and the notion of action of locally compact quantum groups in the $C^{*}$-algebraic setting. We also recall the notion of equivariant Hilbert $C^{*}$-modules (cf. [3]).

- Chapter 3. We make a very brief survey of the theory of measured quantum groupoid (cf. [20, 17]) and we recall the simplified definition in the case where the basis is finitedimensional and the associated $C^{*}$-algebraic structure provided by De Commer in [11, 12]. In the last section, we make an outline of the reflection technique across a Galois object provided by De Commer (cf. [12, 13]), the construction and the structure of the colinking measured quantum groupoid associated with monoidally equivalent locally compact quantum groups. We also recall the precise description of the $\mathrm{C}^{*}$-algebraic structure of colinking measured quantum groupoids (cf. [2]).

- Chapter 4. In this chapter, we make a review of the notions of regularity and semiregularity for measured quantum groupoids on a finite basis (cf. [16, 23, 24, 2]) and we obtain new relations equivalent to the (semi-)regularity generalizing some results of Baaj and Skandalis [4, 1]. Given a (semi-)regular measured quantum groupoid, we derive new relations that will play a crucial role in the subsequent chapters.

- Chapter 5. In the first section of this chapter, we recall the definitions and the main results of [2] concerning the notion of (strongly) continuous action of measured quantum groupoids on a finite basis on $\mathrm{C}^{*}$-algebras. We also recall the version of the Takesaki-Takai duality theorem obtained in [2] in this framework. The second section is dedicated to a brief overview of $\mathrm{C}^{*}$-algebras acted upon by a colinking measured quantum groupoid (cf. [2]). In the last section, we generalize to the setting of measured quantum groupoids on a finite basis the results of Baaj, Skandalis and Vaes [5] concerning the notion of weakly/strongly continuous action of (semi-)regular locally compact quantum groups.

- Chapter 6. We introduce the notion of action of measured quantum groupoid on a finite basis on Hilbert $C^{*}$-module and we investigate in detail the case of a colinking measured quantum groupoid. In the last paragraph, we provide a direct approach of the induction procedure for equivariant Hilbert $C^{*}$-modules equivalent to that obtained in [2]. In particular, if $\mathcal{G}_{\mathrm{G}_{1}, \mathrm{G}_{2}}$ is a colinking measured quantum groupoid associated with two monoidally equivalent regular locally compact groups $\mathrm{G}_{1}$ and $\mathrm{G}_{2}$ we obtain one-to-one 
correspondences between the actions of $\mathrm{G}_{1}, \mathrm{G}_{2}$ and $\mathcal{G}_{\mathrm{G}_{1}, \mathrm{G}_{2}}$ on Hilbert $\mathrm{C}^{*}$-modules.

- Chapter 7. In this chapter, we introduce and discuss the notion of equivariant Morita equivalence. Given a $\mathcal{G}$ - $C^{*}$-algebra $A$, we prove that $A$ and its double crossed product $(A \rtimes \mathcal{G}) \rtimes \widehat{\mathcal{G}}$ are $\mathcal{G}$-equivariantly Morita equivalent in a canonical way.

- Chapter 8 . In the appendix of this article, we have assembled a very brief review of the main notions and notations of the non-commutative measure and integration theory. We can also find some notations and important results used throughout this paper.

In a forthcoming article [10], we use the results of this paper to generalize those of Baaj and Skandalis concerning the equivariant Kasparov theory (cf. $\S 6[3]$ and 7.7 b) [4]).

\section{Acknowledgements}

Some of the results of this article were part of the author's Ph.D. thesis and he wishes to thank his advisor Prof. S. Baaj for his supervision. The author is also very grateful to Prof. K. De Commer for fruitful discussions on measured quantum groupoids and for the financial support of the F.W.O.

\section{PRELIMINARY NOTATIONS}

We specify here some elementary notations and conventions used in this article. For more notations, we refer the reader to the appendix and the index of this article.

- For all subset $X$ of a normed vector space $E$, we denote $\langle X\rangle$ (resp. $[X]$ ) the linear span (resp. closed linear span) of $X$ in $E$. If $X, Y \subset E$, we denote $X Y:=\{x y ; x \in X, y \in Y\}$, where $x y$ denotes the product/composition of $x$ and $y$ or the evaluation of $x$ at $y$ (when these operations make sense). If $X$ is a subset of a ${ }^{*}$-algebra $A$, we denote by $X^{*}$ the subset $\left\{x^{*} ; x \in X\right\}$ of $A$.

- We denote by $\otimes$ the tensor product of Hilbert spaces, the tensor product of von Neumann algebras, the minimal tensor product of $C^{*}$-algebras or the external tensor product of Hilbert $\mathrm{C}^{*}$-modules. We also denote by $\odot\left(\right.$ resp. $\left.\odot_{A}\right)$ the algebraic tensor product over the field of complex numbers $\mathbb{C}$ (resp. an algebra $A$ ).

- Let $A$ and $B$ be $C^{*}$-algebras. We denote by $\mathcal{M}(A)$ (resp. $\widetilde{A}$ ) the $C^{*}$-algebra consisting of the multipliers of $A$ (resp. the $C^{*}$-algebra obtained from $A$ by adjunction of a unit element). We denote by $\widetilde{\mathcal{M}}(A \otimes B)$ (or $\widetilde{\mathcal{M}}_{B}(A \otimes B)$ in case of ambiguity, §1 [3] $)$ the $B$ relative multiplier algebra, i.e. the $C^{*}$-algebra consisting of the elements $m$ of $\mathcal{M}(\widetilde{A} \otimes B)$ such that the relations $(\widetilde{A} \otimes B) m \subset A \otimes B$ and $m(\widetilde{A} \otimes B) \subset A \otimes B$ hold.

Let $\pi: A \rightarrow \mathcal{M}(B)$ be a (possibly degenerate) ${ }^{*}$-homomorphism. For all $C^{*}$-algebra $D$, there exists a unique strictly continuous *-homomorphism $\pi \otimes \operatorname{id}_{D}: \widetilde{\mathcal{M}}(A \otimes D) \rightarrow \mathcal{M}(B \otimes D)$ satisfying the relation $\left(\pi \otimes \operatorname{id}_{D}\right)(x)\left(1_{B} \otimes d\right)=\left(\pi \otimes \operatorname{id}_{D}\right)\left(x\left(1_{A} \otimes d\right)\right)$ for all $x \in \widetilde{\mathcal{M}}(A \otimes D)$ and $d \in D$. Indeed, denote by $\widetilde{\pi}$ the unital extension of $\pi$ to $\widetilde{A}$. The non-degenerate *-homomorphism $\tilde{\pi} \otimes \mathrm{id}_{D}: \widetilde{A} \otimes D \rightarrow \mathcal{M}(B \otimes D)$ uniquely extends to $\mathcal{M}(\widetilde{A} \otimes D)$. By restricting to $\widetilde{\mathcal{M}}(A \otimes D)$, we obtain the desired extension of $\pi \otimes \mathrm{id}_{D}$ (§工 [3]).

- If $x$ and $y$ are two elements of an algebra $A$, we denote by $[x, y]$ the commutator of $x$ and $y$, i.e. the element of $A$ defined by $[x, y]:=x y-y x$.

Let $\mathscr{H}$ and $\mathscr{K}$ be Hilbert spaces (all inner products are assumed to be anti-linear in the first variable and linear in the second variable).

- We denote by $\mathcal{B}(\mathscr{H}, \mathscr{K})$ (resp. $\mathcal{K}(\mathscr{H}, \mathscr{K}))$ the Banach space of bounded (resp. compact) linear operators from $\mathscr{H}$ to $\mathscr{K}$. For all $\xi \in \mathscr{K}$ and $\eta \in \mathscr{H}$, we denote by $\theta_{\xi, \eta} \in \mathcal{B}(\mathscr{H}, \mathscr{K})$ the rank-one operator defined by $\theta_{\xi, \eta}(\zeta):=\langle\eta, \zeta\rangle \xi$ for all $\zeta \in \mathscr{H}$. We have the relation $\mathcal{K}(\mathscr{H}, \mathscr{K})=\left[\theta_{\xi, \eta} ; \xi \in \mathscr{K}, \eta \in \mathscr{K}\right]$. Denote by $\mathcal{B}(\mathscr{H}):=\mathcal{B}(\mathscr{H}, \mathscr{H})($ resp. $\mathcal{K}(\mathscr{H}):=\mathcal{K}(\mathscr{H}, \mathscr{H}))$ the $\mathrm{C}^{*}$-algebra of bounded (resp. compact) linear operators on $\mathscr{H}$. Recall that $\mathcal{K}(\mathscr{H})$ is a closed two-sided ideal of $\mathcal{B}(\mathscr{H})$ and $\mathcal{B}(\mathscr{H})=\mathcal{M}(\mathcal{K}(\mathscr{H}))$.

- We denote by $\Sigma_{\mathscr{K} \otimes \mathscr{H}}$ (or simply $\Sigma$ ) the flip map, that is to say the unitary operator $\mathscr{K} \otimes \mathscr{H} \rightarrow \mathscr{H} \otimes \mathscr{K} ; \xi \otimes \eta \mapsto \eta \otimes \xi$.

- For $u \in \mathcal{B}(\mathscr{H})$, we denote by $\operatorname{Ad}_{u}$ the bounded operator on $\mathcal{B}(\mathscr{H})$ defined for all $x \in \mathcal{B}(\mathscr{H})$ by $\operatorname{Ad}_{u}(x)=u x u^{*}$. 
In this article, we will use the notion of (right) Hilbert $C^{*}$-module over a $C^{*}$-algebra and their tensor products (internal and external). All the definitions and conventions are those of [18]. In particular, let $\mathscr{E}$ and $\mathscr{F}$ be two Hilbert $C^{*}$-modules over a $C^{*}$-algebra $A$.

- We denote by $\mathcal{L}(\mathscr{E}, \mathscr{F})$ the Banach space consisting of all adjointable operators from $\mathscr{E}$ to $\mathscr{F}$ and $\mathcal{L}(\mathscr{E})$ the $\mathrm{C}^{*}$-algebra $\mathcal{L}(\mathscr{E}, \mathscr{E})$.

- For $\xi \in \mathscr{F}$ and $\eta \in \mathscr{E}$, we denote by $\theta_{\tilde{\zeta}, \eta}$ the elementary operator of $\mathcal{L}(\mathscr{E}, \mathscr{F})$ defined by $\theta_{\tilde{\xi}, \eta}(\zeta):=\xi\langle\eta, \zeta\rangle_{A}$ for all $\zeta \in \mathscr{E}$. Let $\mathcal{K}(\mathscr{E}, \mathscr{F}):=\left[\theta_{\tilde{\xi}, \eta} ; \xi \in \mathscr{F}, \eta \in \mathscr{E}\right]$ be the Banach space of "compact" adjointable operators. Denote by $\mathcal{K}(\mathscr{E})$ the $C^{*}$-algebra $\mathcal{K}(\mathscr{E}, \mathscr{E})$ consisting of the compact adjointable operators of $\mathcal{L}(\mathscr{E})$. Recall that $\mathcal{K}(\mathscr{E})$ is a closed two-sided ideal of $\mathcal{L}(\mathscr{E})$ and $\mathcal{L}(\mathscr{E})=\mathcal{M}(\mathcal{K}(\mathscr{E}))$.

- Let $\mathscr{E}^{*}:=\mathcal{K}(\mathscr{E}, A)$. We have $\mathscr{E}^{*}=\left\{T \in \mathcal{L}(\mathscr{E}, A) ; \exists \xi \in \mathscr{E}, \forall \eta \in \mathscr{E}, T(\eta)=\langle\xi, \eta\rangle_{A}\right\}$. We will identify $\mathscr{E}=\mathcal{K}(A, \mathscr{E}) \subset \mathcal{L}(A, \mathscr{E})$. Up to this identification, for $\xi \in \mathscr{E}$ the operator $\xi^{*} \in \mathscr{E}^{*}$ satisfies $\xi^{*}(\eta)=\langle\xi, \eta\rangle_{A}$ for all $\eta \in \mathscr{E}$. We recall that $\mathscr{E}^{*}$ is a Hilbert $\mathcal{K}(\mathscr{E})$-module for the inner product defined by $\left\langle T, T^{\prime}\right\rangle_{\mathcal{K}(\mathscr{E})}:=T^{*} \circ T^{\prime}$ for $T, T^{\prime} \in \mathscr{E}^{*}$ and the right action is defined by the composition of maps.

In this article, we will also use the leg numbering notation. Let $\mathscr{H}$ be a Hilbert space and $T \in \mathcal{B}(\mathscr{H} \otimes \mathscr{H})$. We define the operators $T_{12}, T_{13}, T_{23} \in \mathcal{B}(\mathscr{H} \otimes \mathscr{H})$ by setting $T_{12}:=T \otimes 1$, $T_{23}:=1 \otimes T$ and $T_{13}:=(\Sigma \otimes 1)(1 \otimes T)(\Sigma \otimes 1)$. We can generalize the leg numbering notation for operators acting on any tensor product of Hilbert spaces and for adjointable operators acting on any external tensor product of Hilbert $C^{*}$-modules over possibly different $C^{*}$-algebras.

\section{LOCALLY COMPACT QUANTUM GROUPS}

For the notations and conventions used in this article concerning the non-commutative integration theory and the canonical objects of the Tomita-Takesaki theory, we refer the reader to the appendix of this article (cf. \$8.1).

2.1 Definition. - [19] A locally compact quantum group is a pair $G=\left(L^{\infty}(G), \Delta\right)$, where $\mathrm{L}^{\infty}(\mathrm{G})$ is a von Neumann algebra and $\Delta: \mathrm{L}^{\infty}(\mathrm{G}) \rightarrow \mathrm{L}^{\infty}(\mathrm{G}) \otimes \mathrm{L}^{\infty}(\mathrm{G})$ is a unital normal *-homomorphism satisfying the following conditions:

1. $(\Delta \otimes \mathrm{id}) \Delta=(\mathrm{id} \otimes \Delta) \Delta$;

2. there exist n.s.f. weights $\varphi$ and $\psi$ on $\mathrm{L}^{\infty}(\mathrm{G})$ such that:

(a) $\varphi$ is left invariant, i.e. $\varphi((\omega \otimes$ id $) \Delta(x))=\varphi(x) \omega(1)$, for all $\omega \in \mathrm{L}^{\infty}(\mathbb{G})_{*}^{+}$and $x \in \mathfrak{M}_{\varphi}^{+}$,

(b) $\psi$ is right inveriant, i.e. $\psi((\operatorname{id} \otimes \omega) \Delta(x))=\psi(x) \omega(1)$, for all $\omega \in \mathrm{L}^{\infty}(\mathbb{G})_{*}^{+}$and $x \in \mathfrak{M}_{\psi}^{+}$.

A left (resp. right) invariant n.s.f. weight on $L^{\infty}(\mathbb{G})$ is called a left (resp. right) Haar weight on $\mathrm{G}$.

2.2. For a locally compact quantum group $G$, there exists a unique left (resp. right) Haar weight on $G$ up to a positive scalar [19]. Let us fix a locally compact quantum group $\mathbb{G}:=\left(L^{\infty}(\mathbb{G}), \Delta\right)$. Let us fix a left Haar weight $\varphi$ on $\mathbb{G}$. Let $\left(\mathrm{L}^{2}(\mathbb{G}), \pi, \Lambda\right)$ be the G.N.S. construction for $\left(L^{\infty}(G), \varphi\right)$. The left regular representation of $G$ is the multiplicative unitary [19. 4] $W \in \mathcal{B}\left(\mathrm{L}^{2}(\mathbb{G}) \otimes \mathrm{L}^{2}(\mathbb{G})\right)$ defined by

$$
W^{*}(\Lambda(x) \otimes \Lambda(y))=(\Lambda \otimes \Lambda)(\Delta(y)(x \otimes 1)), \quad \text { for all } x, y \in \mathfrak{N}_{\varphi} .
$$

By identifying $L^{\infty}(\mathbb{G})$ with its image by the G.N.S. representation $\pi$, we obtain:

- $\mathrm{L}^{\infty}(\mathbb{G})$ is the strong closure of the algebra $\left\{(\operatorname{id} \otimes \omega)(W) ; \omega \in \mathcal{B}\left(\mathrm{L}^{2}(\mathbb{G})\right)_{*}\right\}$;

- $\Delta(x)=W^{*}(1 \otimes x) W$, for all $x \in \mathrm{L}^{\infty}(\mathbb{G})$. 
2.3. The Hopf-von Neumann algebra $\left(\mathrm{L}^{\infty}(\mathbb{G}), \Delta\right)$ admits [19] a unitary antipode map $R_{\mathbb{G}}: L^{\infty}(G) \rightarrow L^{\infty}(\mathbb{G})$ and we can choose for right Haar weight on $\mathbb{G}$ the weight $\psi$ defined by $\psi(x):=\varphi\left(R_{\mathbb{G}}(x)\right)$, for all $x \in \mathrm{L}^{\infty}(\mathbb{G})_{+}$. The Connes cocycle derivative [8, 25] of $\psi$ with respect to $\varphi$ is given by

$$
(D \psi: D \varphi)_{t}:=v^{\mathrm{i} t^{2} / 2} d^{\mathrm{i} t}, \quad \text { for all } t \in \mathbb{R},
$$

where $v>0$ is the scaling constant of $\mathbb{G}$ and the operator $d \eta M$ is the modular element of $\mathbb{G}[\overline{19}]$. Let $\mathfrak{N}_{\varphi}^{d}:=\left\{x \in M ; x d^{1 / 2}\right.$ is bounded and its closure $\overline{x d^{1 / 2}}$ belongs to $\left.\mathfrak{N}_{\varphi}\right\}$. The G.N.S. construction [25] for $\left(\mathrm{L}^{\infty}(\mathbb{G}), \psi\right)$ is given by $\left(\mathrm{L}^{2}(\mathbb{G}), \mathrm{id}, \Lambda_{\psi}\right)$, where $\Lambda_{\psi}$ is the closure of the map $\mathfrak{N}_{\varphi}^{d} \rightarrow \mathrm{L}^{2}(\mathbb{G}) ; x \mapsto \Lambda\left(\overline{x d^{1 / 2}}\right)$. We recall that $J_{\psi}=v^{\mathrm{i} / 4} J$, where $J$ denotes the modular conjugation for $\varphi$.

2.4. The right regular representation of the quantum group $G$ is the multiplicative unitary $V \in \mathcal{B}\left(\mathrm{L}^{2}(\mathrm{G}) \otimes \mathrm{L}^{2}(\mathrm{G})\right)$ defined by

$$
V\left(\Lambda_{\psi}(x) \otimes \Lambda_{\psi}(y)\right)=\left(\Lambda_{\psi} \otimes \Lambda_{\psi}\right)(\Delta(x)(1 \otimes y)), \quad \text { for all } x, y \in \mathfrak{N}_{\psi} .
$$

2.5 Definition. - The quantum group $\widehat{G}$ dual of $\mathbb{G}$ is defined by the Hopf-von Neumann algebra $\left(\mathrm{L}^{\infty}(\widehat{\mathrm{G}}), \widehat{\Delta}\right)$, where:

- $\mathrm{L}^{\infty}(\widehat{\mathrm{G}})$ is the strong closure of the algebra $\left\{(\mathrm{id} \otimes \omega)(V) ; \omega \in \mathcal{B}\left(\mathrm{L}^{2}(\mathbb{G})\right\}\right.$;

- the coproduct $\widehat{\Delta}: \mathrm{L}^{\infty}(\widehat{\mathrm{G}}) \rightarrow \mathrm{L}^{\infty}(\widehat{\mathrm{G}}) \otimes \mathrm{L}^{\infty}(\widehat{\mathbb{G}})$ is defined by $\widehat{\Delta}(x):=V^{*}(1 \otimes x) V$ for all $x \in \mathrm{L}^{\infty}(\widehat{\mathrm{G}})$.

The quantum group $\widehat{G}$ admits left and right Haar weights [19] and we can take the Hilbert space $\mathrm{L}^{2}(\mathrm{G})$ for G.N.S. space. We denote by $\widehat{J}$ the modular conjugation of the left Haar weight on $\widehat{G}$.

\subsection{Hopf $C^{*}$-algebras associated with a quantum group}

We associate [4, 19] with the quantum group $G$ two Hopf $C^{*}$-algebras $(S, \delta)$ and $(\widehat{S}, \widehat{\delta})$ defined by:

- $S$ (resp. $\widehat{S})$ is the norm closure of the algebra $\left\{(\omega \otimes \mathrm{id})(V) ; \omega \in \mathcal{B}\left(\mathrm{L}^{2}(\mathbb{G})\right)_{*}\right\}$ (resp. $\left.\left\{(\mathrm{id} \otimes \omega)(V) ; \omega \in \mathcal{B}\left(\mathrm{L}^{2}(\mathrm{G})\right)_{*}\right\}\right)$;

- the coproduct $\delta: S \rightarrow \mathcal{M}(S \otimes S)$ (resp. $\widehat{\delta}: \widehat{S} \rightarrow \mathcal{M}(\widehat{S} \otimes \widehat{S}))$ is given by:

$$
\delta(x):=V(x \otimes 1) V^{*}, \quad \text { for all } x \in S \quad\left(\text { resp. } \widehat{\delta}(x):=V^{*}(1 \otimes x) V, \quad \text { for all } x \in \widehat{S}\right) .
$$

We call $(S, \delta)$ (resp. $(\widehat{S}, \widehat{\delta})$ ) the Hopf $C^{*}$-algebra (resp. dual Hopf $C^{*}$-algebra) associated with $\mathbb{G}$. We can also denote by $C_{0}(\mathbb{G}):=S$ the Hopf $C^{*}$-algebra of $\mathbb{G}$. Note that $C_{0}(\widehat{\mathbb{G}})=\widehat{S}$.

2.1.1 Notations. - - Consider the unitary operator $U:=\widehat{J J} \in \mathcal{B}\left(\mathrm{L}^{2}(\mathbb{G})\right)$. Since $U=v^{\mathrm{i} / 4} J \widehat{J}$, we have $U^{*}=v^{-\mathrm{i} / 4} U$. In particular, $\operatorname{Ad}_{U}=\operatorname{Ad}_{U^{*}}$ on $\mathcal{B}\left(\mathrm{L}^{2}(\mathbb{G})\right)$.

- We have the following non-degenerate faithful representation of the $C^{*}$-algebra $S$ (resp. $\widehat{S}$ ):

$$
\begin{aligned}
& L: S \rightarrow \mathcal{B}\left(\mathrm{L}^{2}(\mathbb{G})\right) ; y \mapsto y ; R: S \rightarrow \mathcal{B}\left(\mathrm{L}^{2}(\mathbb{G})\right) ; y \mapsto U L(y) U^{*} \\
&\text { (resp. } \left.\rho: \widehat{S} \rightarrow \mathcal{B}\left(\mathrm{L}^{2}(\mathbb{G})\right) ; x \mapsto x ; \quad \lambda: \widehat{S} \rightarrow \mathcal{B}\left(\mathrm{L}^{2}(\mathbb{G})\right) ; x \mapsto U \rho(x) U^{*}\right) .
\end{aligned}
$$

It follows from $2.15[19]$ that $W=\Sigma(U \otimes 1) V\left(U^{*} \otimes 1\right) \Sigma$ and $\left[W_{12}, V_{23}\right]=0$. The right regular representation of $\widehat{\mathrm{G}}$ is the multiplicative unitary $\widetilde{V}:=\Sigma(1 \otimes U) V\left(1 \otimes U^{*}\right) \Sigma$.

2.1.2 Notation. - Let $\mathscr{H}$ be a Hilbert space and $X \in \mathcal{B}(\mathscr{H} \otimes \mathscr{H})$. We denote by $\mathcal{C}(X)$ the norm closure of the subspace $\left\{(\mathrm{id} \otimes \omega)(\Sigma X) ; \omega \in \mathcal{B}(\mathscr{H})_{*}\right\}$ of $\mathcal{B}(\mathscr{H})$. If $X$ is a multiplicative unitary, then $\left\{(\mathrm{id} \otimes \omega)(\Sigma X) ; \omega \in \mathcal{B}(\mathscr{H})_{*}\right\}$ is a subalgebra of $\mathcal{B}(\mathscr{H})[4]$.

2.1.3 Definition. - [4, 目] The quantum group $G$ is said to be regular (resp. semi-regular), if $\mathcal{K}\left(\mathrm{L}^{2}(\mathrm{G})\right)=\mathcal{C}(V)\left(\right.$ resp. $\left.\mathcal{K}\left(\mathrm{L}^{2}(\mathrm{G})\right) \subset \mathcal{C}(V)\right)$.

Note that $G$ is regular (resp. semi-regular) if, and only if, $\mathcal{K}\left(\mathrm{L}^{2}(\mathrm{G})\right)=\mathcal{C}(W)$ (resp. $\left.\mathcal{K}\left(\mathrm{L}^{2}(\mathrm{G})\right) \subset \mathcal{C}(W)\right)$. 
We use the notations introduced in the previous paragraph. Let $A$ be a $C^{*}$-algebra.

2.2.1 Definition. - $\quad$ 1. An action of the quantum group $\mathrm{G}$ on $A$ is a non-degenerate *homomorphism $\delta_{A}: A \rightarrow \mathcal{M}(A \otimes S)$ satisfying $\left(\delta_{A} \otimes \mathrm{id}_{S}\right) \delta_{A}=\left(\mathrm{id}_{A} \otimes \delta\right) \delta_{A}$.

2. An action $\delta_{A}$ of $\mathbb{G}$ on $A$ is said to be strongly (resp. weakly) continuous if

$$
\left[\delta_{A}(A)\left(1_{A} \otimes S\right)\right]=A \otimes S \quad\left(\operatorname{resp} . A=\left[\left(\operatorname{id}_{A} \otimes \omega\right) \delta_{A}(A) ; \omega \in \mathcal{B}\left(\mathrm{L}^{2}(\mathrm{G})\right)_{*}\right]\right) .
$$

3. A G-C*-algebra is a pair $\left(A, \delta_{A}\right)$, where $A$ is a $C^{*}$-algebra and $\delta_{A}: A \rightarrow \mathcal{M}(A \otimes S)$ is a strongly continuous action of $\mathbb{G}$ on $A$.

If $\mathbb{G}$ is regular, any weakly continuous action of $\mathbb{G}$ is necessarily continuous in the strong sense, cf. 5.8 [5].

2.2.2 Notations. - Let $\delta_{A}: A \rightarrow \mathcal{M}(A \otimes S)$ (resp. $\delta_{A}: A \rightarrow \mathcal{M}(A \otimes \widehat{S})$ ) be a strongly continuous action of $\mathbb{G}$ (resp. $\widehat{\mathbb{G}}$ ) on the $C^{*}$-algebra $A$. Denote by $\pi_{L}$ (resp. $\widehat{\pi}_{\lambda}$ ) the representation of $A$ on the Hilbert $A$-module $A \otimes \mathrm{L}^{2}(\mathbb{G})$ defined by $\pi_{L}:=\left(\operatorname{id}_{A} \otimes L\right) \delta_{A}$ (resp. $\left.\widehat{\pi}_{\lambda}:=\left(\operatorname{id}_{A} \otimes \lambda\right) \delta_{A}\right)$.

2.2.3 Definition. - (cf. 7.1 [4]) Let $\left(A, \delta_{A}\right)$ be a G-C*-algebra (resp. $\widehat{G}$ - $C^{*}$-algebra). We call (reduced) crossed product of $A$ by $\mathrm{G}$ (resp. $\widehat{\mathrm{G}}$ ), the $\mathrm{C}^{*}$-subalgebra $A \rtimes \mathrm{G}$ (resp. $A \rtimes \widehat{\mathrm{G}}$ ) of $\mathcal{L}\left(A \otimes \mathrm{L}^{2}(\mathbb{G})\right)$ generated by the products $\pi_{L}(a)\left(1_{A} \otimes \rho(x)\right)$ (resp. $\left.\widehat{\pi}_{\lambda}(a)\left(1_{A} \otimes L(x)\right)\right)$ for $a \in A$ and $x \in \widehat{S}$ (resp. $x \in S$ ).

The crossed product $A \rtimes \mathbb{G}$ (resp. $A \rtimes \widehat{\mathbb{G}}$ ) is endowed with a strongly continuous action of $\widehat{G}$ (resp. $G$ ), cf. 7.3 [4]. If $G$ is regular, then the Takesaki-Takai duality extends to this setting, cf. $7 \cdot 5[4]$.

2.2.4 Definition. - Let $A$ and $B$ be two $C^{*}$-algebras. Let $\delta_{A}: A \rightarrow \mathcal{M}(A \otimes S)$ and $\delta_{B}: B \rightarrow \mathcal{M}(B \otimes S)$ be two actions of $\mathbb{G}$ on $A$ and $B$ respectively. A non-degenerate *-homomorphism $f: A \rightarrow \mathcal{M}(B)$ is said to be G-equivariant if $\left(f \otimes \operatorname{id}_{S}\right) \delta_{A}=\delta_{B} \circ f$. We denote by $\mathrm{Alg}_{\mathrm{G}}$ the category whose objects are the $\mathrm{G}-\mathrm{C}^{*}$-algebras and the morphisms are the G-equivariant non-degenerate *-homomorphisms.

\subsection{Equivariant Hilbert $C^{*}$-modules and bimodules}

PRELIMINARIES. In this paragraph, we briefly recall some classical notations and elementary facts concerning Hilbert $C^{*}$-modules. Let $A$ be a $C^{*}$-algebra and $\mathscr{E}$ a Hilbert $A$-module.

2.3.1 Notations. - Let us consider the following maps:

- $\iota_{A}: A \rightarrow \mathcal{K}(\mathscr{E} \oplus A)$, the ${ }^{*}$-homomorphism given by $\iota_{A}(a)(\xi \oplus b)=0 \oplus a b$ for all $a, b \in A$ and $\xi \in \mathscr{E}$;

- $\llcorner\mathscr{E}: \mathscr{E} \rightarrow \mathcal{K}(\mathscr{E} \oplus A)$, the bounded linear map given by $\iota \mathscr{E}(\xi)(\eta \oplus a)=\xi a \oplus 0$ for all $a \in A$ and $\xi, \eta \in \mathscr{E}$;

- $\iota_{\mathscr{E}^{*}}: \mathscr{E}^{*} \rightarrow \mathcal{K}(\mathscr{E} \oplus A)$, the bounded linear map given by $\iota_{\mathscr{E}^{*}}\left(\mathfrak{\zeta}^{*}\right)(\eta \oplus a)=0 \oplus \zeta^{*} \eta$ for all $\xi, \eta \in \mathscr{E}$ and $a \in A$;

- $\iota_{\mathcal{K}(\mathscr{E})}: \mathcal{K}(\mathscr{E}) \rightarrow \mathcal{K}(\mathscr{E} \oplus A)$, the ${ }^{*}$-homomorphism given by $\iota_{\mathcal{K}(\mathscr{E})}(k)(\eta \oplus a)=k \eta \oplus 0$ for all $k \in \mathcal{K}(\mathscr{E}), \eta \in \mathscr{E}$ and $a \in A$.

The result below follows from straightforward computations.

2.3.2 Proposition. - We have the following statements:

1. $\iota_{\mathscr{E}}(\xi a)=\iota_{\mathscr{E}}(\xi) \iota_{A}(a)$ and $\iota_{A}(a) \iota_{\mathscr{E}}\left(\xi^{*}\right)=\iota_{\mathscr{E}}\left(a \xi^{*}\right)$ for all $\xi \in \mathscr{E}$ and $a \in A$; 
2. $\iota_{\mathscr{E}} *\left(\xi^{*}\right)=\iota_{\mathscr{E}}(\xi)^{*}$ and $\iota_{\mathcal{K}(\mathscr{E})}\left(\theta_{\xi, \eta}\right)=\iota_{\mathscr{E}}(\xi) \iota_{\mathscr{E}}(\eta)^{*}$ for all $\xi, \eta \in \mathscr{E}$;

3. $\iota_{\mathscr{E}}(\xi)^{*} \iota_{\mathscr{E}}(\eta)=\iota_{A}(\langle\xi, \eta\rangle)$ for all $\xi, \eta \in \mathscr{E}$;

4. $\mathcal{K}(\mathscr{E} \oplus A)$ is the $C^{*}$-algebra generated by the set $\iota_{A}(A) \cup \iota_{\mathscr{E}}(\mathscr{E})$.

2.3.3 Remarks. - $\quad$ 1. For $a \in A, \xi \in \mathscr{E}$ and $k \in \mathcal{K}(\mathscr{E})$, the operators $\iota_{A}(a), \iota_{\mathscr{E}}(\xi), \iota_{\mathscr{E}} *\left(\xi^{*}\right)$ and $\iota_{\mathcal{K}(\mathscr{E})}(k)$ can be represented by 2 -by-2 matrices acting on the Hilbert $A$-module $\mathscr{E} \oplus A$ as follows:

$$
\iota_{A}(a)=\left(\begin{array}{cc}
0 & 0 \\
0 & a
\end{array}\right) ; \quad \iota_{\mathscr{E}}(\xi)=\left(\begin{array}{cc}
0 & \xi \\
0 & 0
\end{array}\right) ; \quad \iota_{\mathscr{E}} *\left(\xi^{*}\right)=\left(\begin{array}{cc}
0 & 0 \\
\xi^{*} & 0
\end{array}\right) ; \quad \iota_{\mathcal{K}(\mathscr{E})}(k)=\left(\begin{array}{cc}
k & 0 \\
0 & 0
\end{array}\right) .
$$

Moreover, any operator $x \in \mathcal{K}(\mathscr{E} \oplus A)$ can be written in a unique way as follows:

$$
x=\left(\begin{array}{cc}
k & \xi \\
\eta^{*} & a
\end{array}\right), \quad \text { with } k \in \mathcal{K}(\mathscr{E}), \xi, \eta \in \mathscr{E} \text { and } a \in A .
$$

2. Note that $\iota_{A}$ and $\iota_{\mathcal{K}(\mathscr{E})}$ extend uniquely to strictly ${ }^{*}$-strongly continuous unital * homomorphisms $\iota_{A}: \mathcal{M}(A) \rightarrow \mathcal{L}(\mathscr{E} \oplus A)$ and $\iota_{\mathcal{K}(\mathscr{E})}: \mathcal{L}(\mathscr{E}) \rightarrow \mathcal{L}(\mathscr{E} \oplus A)$. Besides, we have $\iota_{A}(m)(\xi \oplus a)=0 \oplus m a$ and $\iota_{\mathcal{K}(\mathscr{E})}(T)(\xi \oplus a)=T \xi \oplus 0$ for all $m \in \mathcal{M}(A)$, $T \in \mathcal{L}(\mathscr{E}), \xi \in \mathscr{E}$ and $a \in A$.

3. $\iota_{\mathscr{E} *}$ admits an extension to a bounded linear map $\iota_{\mathscr{E}} *: \mathcal{L}(\mathscr{E}, A) \rightarrow \mathcal{L}(\mathscr{E} \oplus A)$ in a straightforward way. Similarly, up to the identification $\mathscr{E}=\mathcal{K}(A, \mathscr{E})$, we can also extend $\iota_{\mathscr{E}}$ to a bounded linear map $\iota_{\mathscr{E}}: \mathcal{L}(A, \mathscr{E}) \rightarrow \mathcal{L}(\mathscr{E} \oplus A)$.

4. As in 1 , we can represent the operators $\iota_{A}(m), \iota_{\mathcal{K}(\mathscr{E})}(T), \iota_{\mathscr{E}}(S)$ and $\iota_{\mathscr{E}}\left(S^{*}\right)$, for $m \in \mathcal{M}(A), T \in \mathcal{L}(\mathscr{E})$ and $S \in \mathcal{L}(A, \mathscr{E})$, by 2-by-2 matrices. Moreover, any operator $x \in \mathcal{L}(\mathscr{E} \oplus A)$ can be written in a unique way as follows:

$$
x=\left(\begin{array}{cc}
T & S^{\prime} \\
S^{*} & m
\end{array}\right), \quad \text { with } T \in \mathcal{L}(\mathscr{E}), S, S^{\prime} \in \mathcal{L}(A, \mathscr{E}) \text { and } m \in \mathcal{M}(A) .
$$

By using the matrix notations described above, we derive easily the following useful technical lemma:

2.3.4 Lemma. - Let $x \in \mathcal{L}(\mathscr{E} \oplus A)$ (resp. $x \in \mathcal{K}(\mathscr{E} \oplus A))$. We have:

1. $x \in \iota_{\mathscr{E}}(\mathcal{L}(A, \mathscr{E}))$ (resp. $\left.\iota_{\mathscr{E}}(\mathscr{E})\right)$ if, and only if, $x \iota_{\mathscr{E}}(\xi)=0$ for all $\xi \in \mathscr{E}$ and $\iota_{A}(a) x=0$ for all $a \in A$; in that case, we have $\iota_{A}(m) x=0$ for all $m \in \mathcal{M}(A)$;

2. $x \in \iota_{\mathcal{K}(\mathscr{E})}(\mathcal{L}(\mathscr{E}))\left(\right.$ resp. $\left.\iota_{\mathcal{K}(\mathscr{E})}(\mathcal{K}(\mathscr{E}))\right)$ if, and only if, $x \iota_{A}(a)=0$ and $\iota_{A}(a) x=0$ for all $a \in A$; in that case, we have $x \iota_{A}(m)=\iota_{A}(m) x=0$ for all $m \in \mathcal{M}(A)$.

Let us recall the notion of relative multiplier module, cf. 2.1 [3].

2.3.5 Definition. - Let $A$ and $B$ be two $C^{*}$-algebras and let $\mathscr{E}$ be a Hilbert $C^{*}$-module over $A$. Up to the identification $\mathscr{E} \otimes B=\mathcal{K}(A \otimes B, \mathscr{E} \otimes B)$, we define $\widetilde{\mathcal{M}}(\mathscr{E} \otimes B)$ (or $\widetilde{\mathcal{M}}_{B}(\mathscr{E} \otimes B)$ in case of ambiguity) to be the following subspace of $\mathcal{L}(A \otimes B, \mathscr{E} \otimes B)$ :

$\left\{T \in \mathcal{L}(A \otimes B, \mathscr{E} \otimes B) ; \forall x \in B,\left(1_{\mathscr{E}} \otimes x\right) T \in \mathscr{E} \otimes B\right.$ and $\left.T\left(1_{A} \otimes x\right) \in \mathscr{E} \otimes B\right\}$.

Note that $\widetilde{\mathcal{M}}(\mathscr{E} \otimes B)$ is a Hilbert $C^{*}$-module over $\widetilde{\mathcal{M}}(A \otimes B)$, whose $\widetilde{\mathcal{M}}(A \otimes B)$-valued inner product is given by:

$$
\langle\xi, \eta\rangle:=\xi^{*} \circ \eta, \quad \text { for all } \xi, \eta \in \widetilde{\mathcal{M}}(\mathscr{E} \otimes B) \subset \mathcal{L}(A \otimes B, \mathscr{E} \otimes B) .
$$

Note also that we have $\mathcal{K}(\widetilde{\mathcal{M}}(\mathscr{E} \otimes B)) \subset \widetilde{\mathcal{M}}(\mathcal{K}(\mathscr{E}) \otimes B)$.

2.3.6 Proposition-Definition. - Let $B \subset \mathcal{B}(\mathscr{K})$ be a $C^{*}$-algebra of operators on a Hilbert space $\mathscr{K}$. For all $T \in \mathcal{L}(A \otimes B, \mathscr{E} \otimes B)$ and $\omega \in \mathcal{B}(\mathscr{K})_{*}$, there exists a unique $\left(\operatorname{id}_{\mathscr{E}} \otimes \omega\right)(T) \in \mathcal{L}(A, \mathscr{E})$ such that

$$
\iota_{\mathscr{E}}\left(\mathrm{id}_{\mathscr{E}} \otimes \omega\right)(T)=\left(\operatorname{id}_{\mathcal{K}(\mathscr{E} \oplus A)} \otimes \omega\right)\left(\iota_{\mathscr{E} \otimes B}(T)\right) \in \mathcal{L}(\mathscr{E} \oplus A),
$$

where $\iota_{\mathscr{E} \otimes B}: \mathcal{L}(A \otimes B, \mathscr{E} \otimes B) \rightarrow \mathcal{L}((\mathscr{E} \otimes B) \oplus(A \otimes B))=\mathcal{M}(\mathcal{K}(\mathscr{E} \oplus A) \otimes B)$. If $B \subset \mathcal{B}(\mathscr{K})$ is non-degenerate and $T \in \widetilde{\mathcal{M}}(\mathscr{E} \otimes B)$, then we have $\left(\mathrm{id}_{\mathscr{E}} \otimes \omega\right)(T) \in \mathscr{E}$.

Proof. This is a direct consequence of 2.3.4 1 and the fact that $\iota_{\mathscr{E} \otimes B}(T) \in \widetilde{\mathcal{M}}(\mathcal{K}(\mathscr{E} \oplus A) \otimes B)$ if $T \in \widetilde{\mathcal{M}}(\mathscr{E} \otimes B)$. 
NOTION OF EQUIVARIANT HILBERT $C^{*}$-MODULE. In this paragraph, we recall the notion of equivariant Hilbert $C^{*}$-module through the three equivalent pictures developed

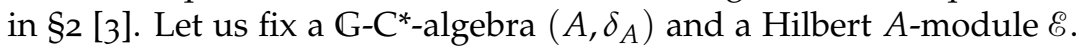

2.3.7 Definition. - An action of the locally compact quantum group $\mathbb{G}$ on $\mathscr{E}$ is a linear map $\delta_{\mathscr{E}}: \mathscr{E} \rightarrow \widetilde{\mathcal{M}}(\mathscr{E} \otimes S)$ such that:

1. $\delta_{\mathscr{E}}(\xi) \delta_{A}(a)=\delta_{\mathscr{E}}(\xi a)$ and $\delta_{A}(\langle\xi, \eta\rangle)=\left\langle\delta_{\mathscr{E}}(\xi), \delta_{\mathscr{E}}(\eta)\right\rangle$, for all $a \in A$ and $\xi, \eta \in \mathscr{E}$;

2. $\left[\delta_{\mathscr{E}}(\mathscr{E})(A \otimes S)\right]=\mathscr{E} \otimes S$;

3. the linear maps $\delta_{\mathscr{E}} \otimes \mathrm{id}_{S}$ and $\mathrm{id}_{\mathscr{E}} \otimes \delta$ extend to linear maps from $\mathcal{L}(A \otimes S, \mathscr{E} \otimes S)$ to

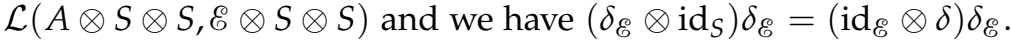

An action $\delta_{\mathscr{E}}$ of $\mathrm{G}$ on $\mathscr{E}$ is said to be continuous if we have $\left[\left(1_{\mathscr{E}} \otimes S\right) \delta_{\mathscr{E}}(\mathscr{E})\right]=\mathscr{E} \otimes S$. A G-equivariant Hilbert $A$-module is a Hilbert $A$-module $\mathscr{E}$ endowed with a continuous action $\delta_{\mathscr{E}}: \mathscr{E} \rightarrow \widetilde{\mathcal{M}}(\mathscr{E} \otimes S)$ of $\mathbb{G}$ on $\mathscr{E}$.

2.3.8. The datum of a continuous action of $G$ on $\mathscr{E}$ is equivalent to that of a continuous action $\delta_{\mathcal{K}(\mathscr{E} \oplus A)}: \mathcal{K}(\mathscr{E} \oplus A) \rightarrow \mathcal{M}(\mathcal{K}(\mathscr{E} \oplus A) \otimes S)$ of $\mathbb{G}$ on the linking $C^{*}$-algebra $\mathcal{K}(\mathscr{E} \oplus A)$ such that the ${ }^{*}$-homomorphism $\iota_{A}: A \rightarrow \mathcal{K}(\mathscr{E} \oplus A)$ is G-equivariant, cf. $2.7[3]$.

2.3.9. If $\delta_{\mathscr{E}}$ is an action of $\mathbb{G}$ on $\mathscr{E}$, we have the unitary operator $\mathcal{V} \in \mathcal{L}\left(\mathscr{E} \otimes \delta_{A}(A \otimes S), \mathscr{E} \otimes S\right)$ defined by $\mathcal{V}\left(\xi \otimes_{\delta_{A}} x\right):=\delta_{\mathscr{E}}(\xi) x$ for all $\xi \in \mathscr{E}$ and $x \in A \otimes S$. This unitary satisfies the relation

$$
\left(\mathcal{V} \otimes_{\mathrm{C}} \mathrm{id}_{S}\right)\left(\mathcal{V} \otimes_{\delta_{A} \otimes \mathrm{id}_{S}} 1\right)=\mathscr{V} \otimes_{\mathrm{id}_{A} \otimes \delta} 1 \quad \text { in } \quad \mathcal{L}\left(\mathscr{E} \otimes_{\delta_{A}^{2}}(A \otimes S \otimes S), \mathscr{E} \otimes S \otimes S\right),
$$

where $\delta_{A}^{2}:=\left(\delta_{A} \otimes \mathrm{id}_{S}\right) \delta_{A}=\left(\operatorname{id}_{A} \otimes \delta\right) \delta_{A}$, cf. 2.3 and 2.4 (a) [3] for the details. Conversely, if there exists a unitary operator $\mathcal{V} \in \mathcal{L}\left(\mathscr{E} \otimes_{\delta_{A}}(A \otimes S), \mathscr{E} \otimes S\right)$ satisfying the above relation and the fact that $\mathcal{V} T_{\xi} \in \widetilde{\mathcal{M}}(\mathscr{E} \otimes S)$ for all $\xi \in \mathscr{E}$, where $T_{\xi} \in \mathcal{L}\left(A \otimes S, \mathscr{E} \otimes \otimes_{A}(A \otimes S)\right)$ is defined by $T_{\xi}(x):=\xi \otimes_{\delta_{A}} x$ for all $x \in A \otimes S$, then the map $\delta_{\mathscr{E}}: \mathscr{E} \rightarrow \widetilde{\mathcal{M}}(\mathscr{E} \otimes S) ; \xi \mapsto \mathcal{V} T_{\xi}$ is an action of $G$ on $\mathscr{E}$, cf. 2.4 (b) [3].

2.3.10. An action of $\mathbb{G}$ on $\mathscr{E}$ determines an action $\delta_{\mathcal{K}(\mathscr{E})}: \mathcal{K}(\mathscr{E}) \rightarrow \widetilde{\mathcal{M}}(\mathcal{K}(\mathscr{E}) \otimes S)$ of $\mathbb{G}$ on $\mathcal{K}(\mathscr{E})$ defined by $\delta_{\mathcal{K}(\mathscr{E})}(k)=\mathcal{V}\left(k \otimes_{\delta_{A}} 1\right)^{*} \mathcal{V}^{*}$ for all $k \in \mathcal{K}(\mathscr{E})$, where $\mathcal{V}$ is the unitary operator associated to the action, cf. 2.8 [3]. Moreover, if $\mathscr{E}$ is a G-equivariant Hilbert module, then $\mathcal{K}(\mathscr{E})$ turns into a $\mathbb{G}-C^{*}$-algebra.

\section{MEASURED QUANTUM GROUPOIDS}

For reminders concerning the relative tensor product of Hilbert spaces and the fiber product of von Neumann algebras, we refer the reader to the appendix of this article (cf. 8.2.

3.1 Definition. - (cf. 3.7 [17], 4.1 [20]) We call a measured quantum groupoid an octuple $\mathcal{G}=\left(N, M, \alpha, \beta, \Gamma, T, T^{\prime}, v\right)$, where:

- $M$ and $N$ are von Neumann algebras;

- $\Gamma: M \rightarrow M_{\beta^{\star} \alpha} M$ is a faithful normal unital *-homomorphism, called the coproduct;

- $\alpha: N \rightarrow M$ and $\beta: N^{\mathrm{o}} \rightarrow M$ are faithful normal unital ${ }^{*}$-homormorphisms, called the range and source maps of $\mathcal{G}$;

- $T: M_{+} \rightarrow \alpha(N)_{+}^{\text {ext }}$ and $T^{\prime}: M_{+} \rightarrow \beta\left(N^{\mathrm{o}}\right)_{+}^{\text {ext }}$ are n.s.f. operator-valued weights;

- $v$ is a n.s.f. weight on $N$;

such that the following conditions are satisfied:

1. $\left[\alpha\left(n^{\prime}\right), \beta\left(n^{\mathrm{o}}\right)\right]=0$, for all $n, n^{\prime} \in N$;

2. $\Gamma(\alpha(n))=\alpha(n)_{\beta} \otimes_{\alpha} 1$ and $\Gamma\left(\beta\left(n^{\circ}\right)\right)=1_{\beta} \otimes_{\alpha} \beta\left(n^{\mathrm{o}}\right)$, for all $n \in N$; 
3. $\Gamma$ is coassociative, i.e. $\left(\Gamma_{\beta \star_{\alpha}} \mathrm{id}\right) \Gamma=\left(\mathrm{id}_{\beta} \star_{\alpha} \Gamma\right) \Gamma$;

4. the n.s.f. weights $\varphi$ and $\psi$ on $M$ given by $\varphi=v \circ \alpha^{-1} \circ T$ and $\psi=v \circ \beta^{-1} \circ T^{\prime}$ satisfy:

- $\forall x \in \mathfrak{M}_{T}^{+}, T(x)=\left(\operatorname{id}_{\beta^{\star} \star_{\alpha}} \varphi\right) \Gamma(x), \quad \forall x \in \mathfrak{M}_{T^{\prime}}^{+}, T^{\prime}(x)=\left(\psi_{\beta \star_{\alpha}} \mathrm{id}\right) \Gamma(x)$,

- $\sigma_{t}^{\varphi}$ and $\sigma_{s}^{\psi}$ commute for all $s, t \in \mathbb{R}$.

Let $\mathcal{G}=\left(N, M, \alpha, \beta, \Gamma, T, T^{\prime}, v\right)$ be a measured quantum groupoid. We denote by $(\mathscr{H}, \pi, \Lambda)$ the G.N.S. construction for $(M, \varphi)$ where $\varphi:=v \circ \alpha^{-1} \circ T$. Let $\left(\sigma_{t}\right)_{t \in \mathbb{R}}, \nabla$ and $J$ be respectively the modular automorphism group, the modular operator and the modular conjugation for $\varphi$. In the following, we identify $M$ with its image by $\pi$ in $\mathcal{B}(\mathscr{H})$.

- We have a coinvolutive *-antiautomomorphism $R_{\mathcal{G}}: M \rightarrow M$ such that $R_{\mathcal{G}}^{2}=\mathrm{id}_{M}$ (cf. 3.8 [17]).

From now on, we will assume that $T^{\prime}=R_{\mathcal{G}} \circ T \circ R_{\mathcal{G}}$ and then also $\psi=\varphi \circ R_{\mathcal{G}}$.

- There exist self-adjoint positive non-singular operators $\lambda$ and $d$ respectively affiliated to $\mathcal{Z}(M)$ and $M$ such that $(D \psi: D \varphi)_{t}=\lambda^{\mathrm{it}{ }^{2} / 2} d^{\mathrm{it}}$ for all $t \in \mathbb{R}$. The operators $\lambda$ and $d$ are respectively called the scaling operator and the modular operator of $\mathcal{G}$.

- The G.N.S. construction for $(M, \psi)$ is given by $\left(\mathscr{H}, \pi_{\psi}, \Lambda_{\psi}\right)$, where: $\Lambda_{\psi}$ is the closure of the operator which sends any element $x \in M$ such that $x d^{1 / 2}$ is closable and its closure $\overline{x d^{1 / 2}} \in \mathfrak{N}_{\varphi}$ to $\Lambda_{\varphi}\left(\overline{x d^{1 / 2}}\right) ; \pi_{\psi}: M \rightarrow \mathcal{B}(\mathscr{H})$ is given by the formula $\pi_{\psi}(a) \Lambda_{\psi}(x)=\Lambda_{\psi}(a x)$.

- The modular conjugation $J_{\psi}$ for $\psi$ is given by $J_{\psi}=\lambda^{\mathrm{i} / 4} J$.

- We will denote by $\mathscr{W}_{\mathcal{G}}: \mathscr{H}_{\beta} \otimes_{\alpha} \mathscr{H} \rightarrow \mathscr{H}_{\alpha} \otimes_{\widehat{\beta}} \mathscr{H}$ the pseudo-multiplicative unitary of $\mathcal{G}$ (cf. 3.3.4 [27], 3.6 [17]).

3.2 Proposition-Definition. - (cf. $3.10[\overline{17}])$ We define the (Pontryagin) dual of $\mathcal{G}$ to be the measured quantum groupoid $\widehat{\mathcal{G}}:=(N, \widehat{M}, \alpha, \widehat{\beta}, \widehat{\Gamma}, \widehat{T}, \widehat{R} \circ \widehat{T} \circ \widehat{R}, v)$, where:

- $\widehat{M}$ is the von Neumann algebra generated by $\left\{(\omega \star \mathrm{id})\left(\mathscr{W}_{\mathcal{G}}\right) ; \omega \in \mathcal{B}(\mathscr{H})_{*}\right\} \subset \mathcal{B}(\mathscr{H})$;

- $\widehat{\beta}: N^{\mathrm{o}} \rightarrow \widehat{M}$ is given by $\widehat{\beta}\left(n^{\mathrm{o}}\right):=J \alpha\left(n^{*}\right) J$ for all $n \in N$;

- $\widehat{\Gamma}: \widehat{M} \rightarrow \widehat{M}_{\widehat{\beta}^{\star \alpha}} \widehat{M}$ is given for all $x \in \widehat{M}$ by $\widehat{\Gamma}(x):=\sigma_{\alpha \widehat{\beta}} W_{\mathcal{G}}\left(x_{\beta} \otimes_{\alpha} 1\right)^{W_{\mathcal{G}}^{*}} \sigma_{\widehat{\beta} \alpha}$;

- there exists a unique n.s.f. weight $\widehat{\varphi}$ on $\widehat{M}$ whose G.N.S. construction is $\left(\mathscr{H}, \mathrm{id}, \Lambda_{\widehat{\varphi}}\right)$, where $\Lambda_{\widehat{\varphi}}$ is the closure of the operator $(\omega \star \mathrm{id})\left(W_{\mathcal{G}}\right) \mapsto a_{\varphi}(\omega)$ defined for normal linear forms $\omega$ in a dense subspace of $\mathscr{I}_{\varphi}=\left\{\omega \in \mathcal{B}(\mathscr{H})_{*} ; \exists k \in \mathbb{R}_{+}, \forall x \in \mathfrak{N}_{\varphi},\left|\omega\left(x^{*}\right)\right|^{2} \leqslant k \varphi\left(x^{*} x\right)\right\}$ and $a_{\varphi}(\omega) \in \mathscr{H}$ satisfies $\omega\left(x^{*}\right)=\left\langle\Lambda_{\varphi}(x), a_{\varphi}(\omega)\right\rangle$ for all $x \in \mathfrak{N}_{\varphi} ;$

- $\widehat{T}$ is the unique n.s.f. operator-valued weight from $\widehat{M}$ to $\alpha(N)$ such that $\widehat{\varphi}=v \circ \alpha^{-1} \circ \widehat{T}$ and $\widehat{T}^{\prime}=R_{\widehat{\mathcal{G}}} \circ \widehat{T} \circ R_{\widehat{\mathcal{G}}}$, where $R_{\widehat{\mathcal{G}}}: \widehat{M} \rightarrow \widehat{M}$ is given by $R_{\widehat{\mathcal{G}}}(x):=J x^{*} J$ for all $x \in \widehat{M}$.

The pseudo-multiplicative unitary $W_{\widehat{\mathcal{G}}}$ of $\widehat{\mathcal{G}}$ is given by $W_{\widehat{\mathcal{G}}}=\sigma_{\beta \alpha} W_{\mathcal{G}}^{*} \sigma_{\widehat{\hat{\beta}} \alpha}$.

We will denote by $\widehat{J}$ the modular conjugation for $\widehat{\varphi}$. Note that the scaling operator of $\widehat{\mathcal{G}}$ is $\lambda^{-1}$. In particular, we have $\lambda^{\mathrm{i} t} \in \mathcal{Z}(M) \cap \mathcal{Z}(\widehat{M})$ for all $t \in \mathbb{R}$.

- Let $\widehat{\alpha}(n):=J \beta\left(n^{\mathrm{o}}\right)^{*} J=\widehat{J} \widehat{\beta}\left(n^{\mathrm{o}}\right)^{*} \widehat{J}$ for $n \in N$. We recall the following relations (cf. 3.11 (v) [17]): $M \cap \widehat{M}=\alpha(N), M \cap \widehat{M}^{\prime}=\beta\left(N^{\mathrm{o}}\right), M^{\prime} \cap \widehat{M}=\widehat{\beta}\left(N^{\mathrm{o}}\right)$ and $M^{\prime} \cap \widehat{M}^{\prime}=\widehat{\alpha}(N)$.

- Let $U:=\widehat{J J} \in \mathcal{B}(\mathscr{H})$. Then, $U^{*}=\lambda^{-i / 4} U$ and $U^{2}=\lambda^{i / 4}$ (cf. 3.11 (iv) [17]). In particular, $U$ is unitary. We have $\widehat{\alpha}(n)=U \alpha(n) U^{*}$ and $\widehat{\beta}\left(n^{\mathrm{o}}\right)=U \beta\left(n^{\mathrm{o}}\right) U^{*}$ for all $n \in N$. Since $\lambda^{-\mathrm{i} / 4} \in \mathcal{Z}(M)$, we also have $\widehat{\alpha}(n)=U^{*} \alpha(n) U$ and $\widehat{\beta}\left(n^{\mathrm{o}}\right)=U^{*} \beta\left(n^{\mathrm{o}}\right) U$ for all $n \in N$.

3.3 Proposition-Definition. - (cf. 3.12 [17]) 
- The octuple $\left(N^{\mathrm{o}}, M, \beta, \alpha, \varsigma_{\beta \alpha} \circ \Gamma, R_{\mathcal{G}} \circ T \circ R_{\mathcal{G}}, T, v^{\mathrm{o}}\right)$ is a measured quantum groupoid denoted by $\mathcal{G}^{\mathrm{o}}$ and called the opposite of $\mathcal{G}$. The pseudo-multiplicative unitary of $\mathcal{G}^{\circ}$ is given by $\mathcal{W}_{\mathcal{G}}=\left({ }_{\beta} \widehat{J}_{\alpha} \otimes_{\widehat{\alpha}} \widehat{J}_{\widehat{\beta}}\right) W_{\mathcal{G}}\left({ }_{\beta} \widehat{J}_{\alpha} \otimes_{\alpha} \widehat{J}_{\beta}\right)$.

- Let $C_{M}: M \rightarrow M^{\prime}$ be the canonical ${ }^{*}$-antihomomorphism given by $C_{M}(x):=J x^{*} J$ for all $x \in M$. Let us define:

$$
\Gamma^{\mathrm{c}}:=\left(C_{M \beta^{\star}{ }_{\alpha}} C_{M}\right) \circ \Gamma \circ C_{M}^{-1} ; \quad R_{\mathcal{G}}^{\mathrm{c}}:=C_{M} \circ R_{\mathcal{G}} \circ C_{M}^{-1} ; \quad T^{\mathrm{c}}=C_{M} \circ T \circ C_{M}^{-1} .
$$

Then, the octuple $\left(N^{\mathrm{o}}, M^{\prime}, \widehat{\beta}, \widehat{\alpha}, \Gamma^{\mathrm{c}}, T^{\mathrm{c}}, R_{\mathcal{G}}^{\mathrm{c}} T^{\mathrm{c}} R_{\mathcal{G}}^{\mathrm{c}}, v^{\mathrm{o}}\right)$ is a measured quantum groupoid denoted by $\mathcal{G}^{\mathrm{c}}$ and called the commutant of $\mathcal{G}$. The pseudo-multiplicative unitary ${ }^{W_{\mathcal{G}}}$ of $\mathcal{G}^{\mathrm{c}}$ is given by $W_{\mathcal{G}^{c}}=\left({ }_{\widehat{\beta}} J_{\alpha} \otimes_{\alpha} J_{\widehat{\beta}}\right)^{2} W_{\mathcal{G}}\left({ }_{\beta} J_{\widehat{\alpha}} \otimes_{\alpha} J_{\widehat{\beta}}\right)$.

3.4 Notations. - For a given measured quantum groupoid $\mathcal{G}$, we will need the following pseudo-multiplicative unitaries:

$$
\widehat{\mathcal{V}}:=W_{\mathcal{G}} ; \quad \mathcal{V}:=W_{\widehat{\left(\mathcal{G}^{\circ}\right)}}=W_{(\widehat{\mathcal{G}})^{c}} ; \quad \widetilde{\mathcal{V}}:=W_{\left(\mathcal{G}^{\circ}\right)^{c}} .
$$

3.5 Convention. - Henceforth, we will refer to $(\widehat{\mathcal{G}})^{\mathrm{c}}$ instead of $\widehat{\mathcal{G}}$ as the dual of $\mathcal{G}$ since this groupoid is better suited for right actions of $\mathcal{G}$. We have

$$
(\widehat{\mathcal{G}})^{\mathrm{c}}=\left(N^{\mathrm{o}}, \widehat{M}^{\prime}, \beta, \widehat{\alpha}, \widehat{\Gamma}^{\mathrm{c}}, \widehat{T}^{\mathrm{c}}, \widehat{T}^{\mathrm{c}}, v^{\mathrm{o}}\right),
$$

where the coproduct and the operator-valued weights are given by:

- $\widehat{\Gamma}^{\mathrm{c}}(x)=\left(\mathscr{W}_{(\widehat{\mathcal{G}})^{\mathrm{c}}}\right)^{*}\left(1_{\beta} \otimes_{\alpha} x\right)^{\left.W_{(\widehat{\mathcal{G}}}\right)^{\mathrm{c}}}$, for all $x \in \widehat{M}^{\prime} ;$

- $\widehat{T}^{\mathrm{c}}=C_{\widehat{M}} \circ \widehat{T} \circ C_{\widehat{M}}^{-1}$, where $C_{\widehat{M}}: \widehat{M} \rightarrow \widehat{M}^{\prime} ; x \mapsto \widehat{J} x^{*} \widehat{J}$;

- $\widehat{T}^{\mathrm{c} \prime}=R_{(\widehat{\mathcal{G}})^{\mathrm{c}}} \circ \widehat{T}^{\mathrm{c}} \circ R_{(\widehat{\mathcal{G}})^{\mathrm{c}}}$.

Note also that the commutant weight $\widehat{\varphi}^{\mathrm{c}}:=v^{\mathrm{o}} \circ \beta^{-1} \circ \widehat{T}^{\mathrm{c}}$ derived from the weight $\widehat{\varphi}$ is left invariant for the coproduct $\widehat{\Gamma}^{c}$. In the following, we will simply denote by $\widehat{\mathcal{G}}$ the dual groupoid of $\mathcal{G}$ (since no ambiguity will arise with the Pontryagin dual). Note that the bidual groupoid is $\left(\mathcal{G}^{\mathrm{o}}\right)^{\mathrm{c}}=\left(\mathcal{G}^{\mathrm{c}}\right)^{\mathrm{o}}$.

\subsection{Case where the basis is finite-dimensional}

In [12], De Commer provides an equivalent definition of a measured quantum groupoid on a finite basis. This definition is far more tractable since it allows us to circumvent the use of relative tensor products and fiber products.

In the following, we fix a finite-dimensional $\mathrm{C}^{*}$-algebra $N:=\bigoplus_{1 \leqslant l \leqslant k} \mathrm{M}_{n_{l}}(\mathbb{C})$ endowed with the non-normalized Markov trace $\epsilon:=\bigoplus_{1 \leqslant l \leqslant k} n_{l} \cdot \operatorname{Tr}_{l}$, where $\operatorname{Tr}_{l}$ denotes the nonnormalized trace on $\mathrm{M}_{n_{l}}(\mathbb{C})$.

We refer to 88.2 of the appendix for the definitions of $v_{\beta \alpha}$ and $q_{\beta \alpha}$. Let us a fix a measured quantum groupoid $\mathcal{G}=\left(N, M, \alpha, \beta, \Gamma, T, T^{\prime}, \epsilon\right)$. We have a unital normal ${ }^{*}$-isomorphism $M_{\beta \star_{\alpha}} M \rightarrow q_{\beta \alpha}(M \otimes M) q_{\beta \alpha} ; x \mapsto v_{\beta \alpha}^{*} x v_{\beta \alpha}$ (cf. 8.2.14). Let $\Delta: M \rightarrow M \otimes M$ be the (non necessarily unital) faithful normal *-homomorphism given by $\Delta(x)=v_{\beta \alpha}^{*} \Gamma(x) v_{\beta \alpha}$ for all $x \in M$. We have $\Delta(1)=q_{\beta \alpha}$. This has led De Commer to the following equivalent definition of a measured quantum groupoid on a finite basis.

3.1.1 Definition. - (cf. 11.1.2 [12]) A measured quantum groupoid on the finite-dimensional basis $N$ is an octuple $\mathcal{G}=\left(N, M, \alpha, \beta, \Delta, T, T^{\prime}, \epsilon\right)$, where:

- $M$ is a von Neumann algebra, $\alpha: N \rightarrow M$ and $\beta: N^{0} \rightarrow M$ are unital faithful normal *-homomorphisms;

- $\Delta: M \rightarrow M \otimes M$ is a faithful normal ${ }^{*}$-homomorphism; 
- $T: M_{+} \rightarrow \alpha(N)_{+}^{\text {ext }}$ and $T^{\prime}: M_{+} \rightarrow \beta\left(N^{\mathrm{o}}\right)_{+}^{\text {ext }}$ are n.s.f. operator-valued weights;

such that the following conditions are satisfied:

1. $\left[\alpha\left(n^{\prime}\right), \beta\left(n^{\mathrm{o}}\right)\right]=0$, for all $n, n^{\prime} \in N$;

2. $\Delta(1)=q_{\beta \alpha}$;

3. $(\Delta \otimes \mathrm{id}) \Delta=(\mathrm{id} \otimes \Delta) \Delta$;

4. $\Delta(\alpha(n))=\Delta(1)(\alpha(n) \otimes 1)$ and $\Delta\left(\beta\left(n^{\mathrm{o}}\right)\right)=\Delta(1)\left(1 \otimes \beta\left(n^{\mathrm{o}}\right)\right)$, for all $n \in N$;

5. the n.s.f. weights $\varphi$ and $\psi$ on $M$ given by $\varphi:=\epsilon \circ \alpha^{-1} \circ T$ and $\psi:=\epsilon \circ \beta^{-1} \circ T^{\prime}$ satisfy:

$$
T(x)=(\mathrm{id} \otimes \varphi) \Delta(x) \quad \text { for all } x \in \mathfrak{M}_{T}^{+}, \quad T^{\prime}(x)=(\psi \otimes \mathrm{id}) \Delta(x), \quad \text { for all } x \in \mathfrak{M}_{T^{\prime}}^{+} ;
$$

6. $\sigma_{t}^{T} \circ \beta=\beta$ and $\sigma_{t}^{T^{\prime}} \circ \alpha=\alpha$, for all $t \in \mathbb{R}$.

Let us fix a measured quantum groupoid $\mathcal{G}=\left(N, M, \alpha, \beta, \Delta, T, T^{\prime}, \epsilon\right)$.

3.1.2 Notations. - Let us consider the injective bounded linear map

$$
\iota_{\widehat{\alpha} \alpha}^{\beta}: \mathcal{B}\left(\mathscr{H}_{\widehat{\alpha}} \otimes_{\beta} \mathscr{H}, \mathscr{H}_{\beta} \otimes_{\alpha} \mathscr{H}\right) \rightarrow \mathcal{B}(\mathscr{H} \otimes \mathscr{H}) \quad ; \quad X \mapsto v_{\beta \alpha}^{*} X v_{\widehat{\alpha} \beta} .
$$

Similarly, we also define $\iota_{\beta \widehat{\beta}}^{\alpha}$ and $\iota_{\widehat{\beta} \beta}^{\widehat{\alpha}}$. Let

$$
V:=\iota_{\widehat{\alpha} \alpha}^{\beta}(\mathcal{V}), \quad W:=l_{\beta \widehat{\beta}}^{\alpha}(\widehat{\mathcal{V}}) \text { and } \quad \widetilde{V}:=\iota_{\widehat{\beta} \beta}^{\widehat{\alpha}}(\widetilde{\mathcal{V}}),
$$

where $\mathcal{V}=\mathscr{W}_{\widehat{\mathcal{G}}}, \widehat{\mathcal{V}}=\mathscr{W}_{\mathcal{G}}$ and $\widetilde{\mathcal{V}}=\mathscr{W}_{(\mathcal{G}}$ oc $($ cf. 3.4).

In what follows, we recall the main properties satisfied by $V, W$ and $\widetilde{V}$. The proof of the results below are derived from the properties satisfied by the pseudo-multiplicative unitaries $\mathcal{V}, \widehat{\mathcal{V}}$ and $\widetilde{\mathcal{V}}$ (cf. [17], §11 [12] and §2 [2]).

3.1.3 Proposition. - (cf. 3.11 (iii), $3.12(v)$, (vi) [17], 2.2 [2]) The operators $V, W$ and $\widetilde{V}$ are multiplicative partial isometries acting on $\mathscr{H} \otimes \mathscr{H}$ such that:

1. $W=\Sigma(U \otimes 1) V\left(U^{*} \otimes 1\right) \Sigma, \quad \widetilde{V}=\Sigma(1 \otimes U) V\left(1 \otimes U^{*}\right) \Sigma=(U \otimes U) W\left(U^{*} \otimes U^{*}\right) ;$

2. $V^{*}=(J \otimes \widehat{J}) V(J \otimes \widehat{J}), \quad W^{*}=(\widehat{J} \otimes J) W(\widehat{J} \otimes J)$;

3. the initial and final projections are given by

$$
V^{*} V=q_{\widehat{\alpha} \beta}=\widetilde{V} \widetilde{V}^{*}, \quad W^{*} W=q_{\beta \alpha}=V V^{*}, \quad W W^{*}=q_{\alpha \widehat{\beta}} \quad \text { and } \quad \widetilde{V}^{*} \widetilde{V}=q_{\widehat{\beta} \widehat{\alpha}} .
$$

3.1.4 Proposition. - (cf. 3.8, 3.12 [17])

1. The von Neumann algebra $M($ resp. $\widehat{M})$ is the weak closure of $\left\{(\mathrm{id} \otimes \omega)(W) ; \omega \in \mathcal{B}(\mathscr{H})_{*}\right\}$ (resp. $\left.\left\{(\omega \otimes \mathrm{id})(W) ; \omega \in \mathcal{B}(\mathscr{H})_{*}\right\}\right)$.

2. We have $W \in M \otimes \widehat{M}, V \in \widehat{M}^{\prime} \otimes M$, and $\widetilde{V} \in M^{\prime} \otimes \widehat{M}^{\prime}$. In particular, we have the commutation relations $\left[W_{12}, V_{23}\right]=0$ and $\left[V_{12}, \widetilde{V}_{23}\right]=0$.

3. The coproduct $\Delta: M \rightarrow M \otimes M$ of $\mathcal{G}$ (resp. $\widehat{\Delta}: \widehat{M}^{\prime} \rightarrow \widehat{M}^{\prime} \otimes \widehat{M}^{\prime}$ of $\widehat{\mathcal{G}}$ ) satisfies

$$
\begin{aligned}
\Delta(x) & =V(x \otimes 1) V^{*}=W^{*}(1 \otimes x) W, \quad \text { for all } x \in M \\
(\operatorname{resp} . \widehat{\Delta}(x) & \left.=V^{*}(1 \otimes x) V=\widetilde{V}(x \otimes 1) \widetilde{V}^{*}, \quad \text { for all } x \in \widehat{M}^{\prime}\right) .
\end{aligned}
$$

3.1.5 Proposition. - (cf. 3.2. (i), 3.6. (ii) [17] and 11.1.2 [12]) For all $n \in N$, we have:

1. $[V, \alpha(n) \otimes 1]=0, \quad\left[V, \widehat{\beta}\left(n^{\mathrm{o}}\right) \otimes 1\right]=0, \quad[V, 1 \otimes \widehat{\alpha}(n)]=0, \quad\left[V, 1 \otimes \widehat{\beta}\left(n^{\mathrm{o}}\right)\right]=0 ;$

2. $V(1 \otimes \alpha(n))=(\widehat{\alpha}(n) \otimes 1) V, \quad V\left(\beta\left(n^{\mathrm{o}}\right) \otimes 1\right)=\left(1 \otimes \beta\left(n^{\mathrm{o}}\right)\right) V ;$ 
3. $\left[W, \widehat{\beta}\left(n^{\mathrm{o}}\right) \otimes 1\right]=0, \quad[W, \widehat{\alpha}(n) \otimes 1]=0, \quad\left[W, 1 \otimes \beta\left(n^{\mathrm{o}}\right)\right]=0, \quad[W, 1 \otimes \widehat{\alpha}(n)]=0$;

4. $W\left(1 \otimes \widehat{\beta}\left(n^{\mathrm{o}}\right)\right)=\left(\beta\left(n^{\mathrm{o}}\right) \otimes 1\right) W, \quad W(\alpha(n) \otimes 1)=(1 \otimes \alpha(n)) W$;

5. $[\widetilde{V}, \alpha(n) \otimes 1]=0, \quad\left[\widetilde{V}, \beta\left(n^{\circ}\right) \otimes 1\right]=0, \quad[\widetilde{V}, 1 \otimes \alpha(n)]=0, \quad\left[\widetilde{V}, 1 \otimes \widehat{\beta}\left(n^{\circ}\right)\right]=0$;

6. $\widetilde{V}\left(1 \otimes \beta\left(n^{\mathrm{o}}\right)\right)=\left(\widehat{\beta}\left(n^{\mathrm{o}}\right) \otimes 1\right) \widetilde{V}, \quad \widetilde{V}(\widehat{\alpha}(n) \otimes 1)=(1 \otimes \widehat{\alpha}(n)) \widetilde{V}$.

3.1.6 Proposition. - (cf. 11.1.4 [12]) For all $n \in N$, we have:

1. $W\left(\beta\left(n^{\mathrm{o}}\right) \otimes 1\right)=W(1 \otimes \alpha(n)), \quad\left(1 \otimes \widehat{\beta}\left(n^{\mathrm{o}}\right)\right) W=(\alpha(n) \otimes 1) W$;

2. $V\left(1 \otimes \beta\left(n^{\mathrm{o}}\right)\right)=V(\widehat{\alpha}(n) \otimes 1), \quad(1 \otimes \alpha(n)) V=\left(\beta\left(n^{\mathrm{o}}\right) \otimes 1\right) V$;

3. $\widetilde{V}\left(\widehat{\beta}\left(n^{\mathrm{o}}\right) \otimes 1\right)=\widetilde{V}(1 \otimes \widehat{\alpha}(n)), \quad\left(1 \otimes \beta\left(n^{\mathrm{o}}\right)\right) \widetilde{V}=(\widehat{\alpha}(n) \otimes 1) \widetilde{V}$.

3.2 Weak Hopf $C^{*}$-algebras associated with a measured quantum groupoid on a finite basis

We recall the definitions and the main results concerning the weak Hopf $\mathrm{C}^{*}$-algebras associated with a measured quantum groupoid on a finite basis, cf. §11.2 [12] (with different notations and conventions, cf. §2.3 [2]). Let us fix a measured quantum groupoid $\mathcal{G}=\left(N, M, \alpha, \beta, \Delta, T, T^{\prime}, \epsilon\right)$ on the finite-dimensional basis $N=\bigoplus_{1 \leqslant l \leqslant k} \mathrm{M}_{n_{l}}(\mathbb{C})$.

3.2.1 Notations. - With the notations of $\$ 3.1$. we denote by $S$ (resp. $\widehat{S}$ ) the norm closure of the subalgebra

$$
\left\{(\omega \otimes \mathrm{id})(V) ; \omega \in \mathcal{B}(\mathscr{H})_{*}\right\} \quad\left(\operatorname{resp} .\left\{(\mathrm{id} \otimes \omega)(V) ; \omega \in \mathcal{B}(\mathscr{H})_{*}\right\}\right) .
$$

According to $\$ 11.2$ [12], we have the following statements:

- the Banach space $S$ (resp. $\widehat{S}$ ) is a non-degenerate $C^{*}$-subalgebra of $\mathcal{B}(\mathscr{H})$, weakly dense in $M$ (resp. $\left.\widehat{M}^{\prime}\right)$;

- the $C^{*}$-algebra $S$ (resp. $\widehat{S}$ ) is endowed with the faithful non-degenerate ${ }^{*}$-representations:

$$
\begin{aligned}
L: S \rightarrow \mathcal{B}(\mathscr{H}) ; x \mapsto x ; & R: S \rightarrow \mathcal{B}(\mathscr{H}) ; x \mapsto U L(x) U^{*} \\
\text { (resp. } \rho: \widehat{S} \rightarrow \mathcal{B}(\mathscr{H}) ; x \mapsto x ; & \left.\lambda: \widehat{S} \rightarrow \mathcal{B}(\mathscr{H}) ; x \mapsto U \rho(x) U^{*}\right) ;
\end{aligned}
$$

- $\alpha(N) \subset \mathcal{M}(S), \beta\left(N^{\mathrm{o}}\right) \subset \mathcal{M}(S), \beta\left(N^{\mathrm{o}}\right) \subset \mathcal{M}(\widehat{S})$ and $\widehat{\alpha}(N) \subset \mathcal{M}(\widehat{S})$;

- $V \in \mathcal{M}(\widehat{S} \otimes S), W \in \mathcal{M}(S \otimes \lambda(\widehat{S}))$ and $\widetilde{V} \in \mathcal{M}(R(S) \otimes \widehat{S})$;

- $\Delta$ (resp. $\widehat{\Delta})$ restricts to a strictly continuous ${ }^{*}$-homomorphism $\delta: S \rightarrow \mathcal{M}(S \otimes S)$ (resp. $\widehat{\delta}: \widehat{S} \rightarrow \mathcal{M}(\widehat{S} \otimes \widehat{S}))$, which uniquely extends to a strictly continuous *-homomorphism $\delta: \mathcal{M}(S) \rightarrow \mathcal{M}(S \otimes S)\left(\right.$ resp. $\widehat{\delta}: \mathcal{M}(\widehat{S}) \rightarrow \mathcal{M}(\widehat{S} \otimes \widehat{S})$ ) satisfying $\delta\left(1_{S}\right)=q_{\beta \alpha}$ (resp. $\left.\widehat{\delta}\left(1_{\widehat{S}}\right)=q_{\widehat{\alpha} \beta}\right)$;

- $\delta($ resp. $\widehat{\delta})$ is coassociative and satisfies $\left[\delta(S)\left(1_{S} \otimes S\right)\right]=\delta\left(1_{S}\right)(S \otimes S)=\left[\delta(S)\left(S \otimes 1_{S}\right)\right]$ $\left(\operatorname{resp} \cdot\left[\widehat{\delta}(\widehat{S})\left(1_{\widehat{S}} \otimes \widehat{S}\right)\right]=\widehat{\delta}\left(1_{\widehat{S}}\right)(\widehat{S} \otimes \widehat{S})=\left[\widehat{\delta}(\widehat{S})\left(\widehat{S} \otimes 1_{\widehat{S}}\right)\right]\right) ;$

- the unital faithful *-homomorphisms $\alpha: N \rightarrow \mathcal{M}(S)$ and $\beta: N^{o} \rightarrow \mathcal{M}(S)$ satisfy

$$
\delta(\alpha(n))=\delta\left(1_{S}\right)\left(\alpha(n) \otimes 1_{S}\right) \text { and } \delta\left(\beta\left(n^{\mathrm{o}}\right)\right)=\delta\left(1_{S}\right)\left(1_{S} \otimes \beta\left(n^{\mathrm{o}}\right)\right), \text { for all } n \in N ;
$$

- the unital faithful *-homomorphisms $\beta: N^{\mathrm{o}} \rightarrow \mathcal{M}(\widehat{S})$ and $\widehat{\alpha}: N \rightarrow \mathcal{M}(\widehat{S})$ satisfy

$$
\widehat{\delta}\left(\beta\left(n^{\mathrm{o}}\right)\right)=\widehat{\delta}\left(1_{\widehat{S}}\right)\left(\beta\left(n^{\mathrm{o}}\right) \otimes 1_{\widehat{S}}\right) \text { and } \widehat{\delta}(\widehat{\alpha}(n))=\widehat{\delta}\left(1_{\widehat{S}}\right)\left(1_{\widehat{S}} \otimes \widehat{\alpha}(n)\right) \text {, for all } n \in N \text {. }
$$

3.2.2 Definition. - With the above notations, we call the pair $(S, \delta)$ (resp. $(\widehat{S}, \widehat{\delta}))$ the weak Hopf $C^{*}$-algebra (resp. dual weak Hopf $C^{*}$-algebra) associated with the measured quantum groupoid $\mathcal{G}$.

3.2.3 REMARK. - With the notations of the above definition, the pair $(\widehat{S}, \widehat{\delta})$ is the weak Hopf $C^{*}$-algebra of $\widehat{\mathcal{G}}$ while its dual weak Hopf $C^{*}$-algebra is the pair $\left(R(S), \delta_{R}\right)$, where $R(S)=U S U^{*}$ and the coproduct $\delta_{R}$ is given by $\delta_{R}(y):=\widetilde{V}^{*}(1 \otimes y) \widetilde{V}$ for all $y \in R(S)$. 


\subsection{Measured quantum groupoid associated with a monoidal equivalence}

We will recall the construction of the measured quantum groupoid associated with a monoidal equivalence between two locally compact quantum groups provided by De Commer [12, 13]. First of all, we will need to recall the definitions and the crucial results of De Commer [12, 13].

3.3.1 Definition. - Let $G$ be a locally compact quantum group. A right (resp. left) Galois action of $\mathbb{G}$ on a von Neumann algebra $N$ is an ergodic integrable right (resp. left) action $\alpha_{N}: N \rightarrow N \otimes L^{\infty}(\mathbb{G})\left(\right.$ resp. $\gamma_{N}: N \rightarrow L^{\infty}(\mathbb{G}) \otimes N$ ) such that the crossed product $N \rtimes_{\alpha_{N}} \mathbb{G}$ (resp. $\left.\mathbb{G}_{\gamma_{N}} \ltimes N\right)$ is a type I factor. Then, the pair $\left(N, \alpha_{N}\right)\left(\operatorname{resp} .\left(N, \gamma_{N}\right)\right)$ is called a right (resp. left) Galois object for $\mathrm{G}$.

Let $\mathbb{G}$ be a locally compact quantum group and let us fix a right Galois object $\left(N, \alpha_{N}\right)$ for $\mathbb{G}$. In his thesis, De Commer was able to build a locally compact quantum group $\mathbb{H}$ equipped with a left Galois action $\gamma_{N}$ on $N$ commuting with $\alpha_{N}$, i.e. $\left(\right.$ id $\left.\otimes \alpha_{N}\right) \gamma_{N}=\left(\gamma_{N} \otimes\right.$ id $) \alpha_{N}$. This construction is called the reflection technique and $\mathbb{H}$ is called the reflected locally compact quantum group across $\left(N, \alpha_{N}\right)$.

In a canonical way, he was also able to associate a right Galois object $\left(O, \alpha_{O}\right)$ for $\mathbb{H}$ and a left Galois action $\gamma_{O}: O \rightarrow \mathrm{L}^{\infty}(\mathbb{G}) \otimes O$ of $\mathbb{G}$ on $O$ commuting with $\alpha_{O}$. Finally, De Commer has built a measured quantum groupoid

$$
\mathcal{G}_{\mathbb{H}, \mathrm{G}}=\left(\mathbb{C}^{2}, M, \alpha, \beta, \Delta, T, T^{\prime}, \epsilon\right)
$$

where: $M=\mathrm{L}^{\infty}(\mathbb{H}) \oplus N \oplus O \oplus \mathrm{L}^{\infty}(\mathrm{G}) ; \Delta: M \rightarrow M \otimes M$ is made up of the coactions and coproducts of the constituents of $M$; the operator-valued weights $T$ and $T^{\prime}$ are given by the invariants weights; the non-normalized Markov trace $\epsilon$ on $\mathbb{C}^{2}$ is simply given by $\epsilon(a, b)=a+b$ for all $(a, b) \in \mathbb{C}^{2}$. Moreover, the source and target maps $\alpha$ and $\beta$ have range in $\mathcal{Z}(M)$ and generate a copy of $\mathbb{C}^{4}$.

Conversely, if $\mathcal{G}=\left(\mathbb{C}^{2}, M, \alpha, \beta, \Delta, T, T^{\prime}, \epsilon\right)$ is a measured quantum groupoid whose source and target maps have range in $\mathcal{Z}(M)$ and generate a copy of $\mathbb{C}^{4}$, then $\mathcal{G}$ is of the form $\mathcal{G}_{\mathbb{H}, \mathrm{G}}$ in a unique way, where $\mathbb{H}$ and $\mathrm{G}$ are locally compact quantum groups canonically associated with $\mathcal{G}$.

In what follows, we fix a measured quantum groupoid $\mathcal{G}=\left(\mathbb{C}^{2}, M, \alpha, \beta, \Delta, T, T^{\prime}, \epsilon\right)$ whose source and target maps have range in $\mathcal{Z}(M)$ and generate a copy of $\mathbb{C}^{4}$. It is worth noticing that for such a groupoid we have:

3.3.2 Lemma. $-(c f .2 .21[2]) \widehat{\alpha}=\beta$ and $\widehat{\beta}=\alpha$.

Following the notations introduced in [12], we recall the precise description of the left and right regular representations $W$ and $V$ of $\mathcal{G}$ introduced in the previous section. We identify $M$ with its image by $\pi$ in $\mathcal{B}(\mathscr{H})$, where $(\mathscr{H}, \pi, \Lambda)$ is the G.N.S. construction for $M$ endowed with the n.s.f. weight $\varphi=\epsilon \circ \alpha^{-1} \circ T$. We also consider the n.s.f. weight $\psi=\epsilon \circ \beta^{-1} \circ T^{\prime}$. Denote by $\left(\varepsilon_{1}, \varepsilon_{2}\right)$ the standard basis of the vector space $\mathbb{C}^{2}$.

3.3.3 Notations. - Let us introduce some useful notations and make some remarks concerning them.

- For $i, j=1,2$, we define the following nonzero central self-adjoint projection of $M$ :

$$
p_{i j}:=\alpha\left(\varepsilon_{i}\right) \beta\left(\varepsilon_{j}\right) .
$$

It follows from $\beta\left(\varepsilon_{1}\right)+\beta\left(\varepsilon_{2}\right)=1_{M}$ and $\alpha\left(\varepsilon_{1}\right)+\alpha\left(\varepsilon_{2}\right)=1_{M}$ that

$$
\alpha\left(\varepsilon_{i}\right)=p_{i 1}+p_{i 2} \quad \text { and } \beta\left(\varepsilon_{j}\right)=p_{1 j}+p_{2 j}, \quad \text { for all } i, j=1,2 .
$$

- We have

$$
\Delta(1)=\alpha\left(\varepsilon_{1}\right) \otimes \beta\left(\varepsilon_{1}\right)+\alpha\left(\varepsilon_{2}\right) \otimes \beta\left(\varepsilon_{2}\right) \text { and } \widehat{\Delta}(1)=\beta\left(\varepsilon_{1}\right) \otimes \beta\left(\varepsilon_{1}\right)+\beta\left(\varepsilon_{2}\right) \otimes \beta\left(\varepsilon_{2}\right)
$$

since $\widehat{\alpha}=\beta$. 
- Let $M_{i j}:=p_{i j} M$, for $i, j=1,2$. Then, $M_{i j}$ is a nonzero von Neumann subalgebra of M.

- Let $\mathscr{H}_{i j}:=p_{i j} \mathscr{H}$, for $i, j=1,2$. Then, $\mathscr{H}_{i j}$ is a nonzero Hilbert subspace of $\mathscr{H}$ for all $i, j=1,2$.

- Let $\varphi_{i j}:=\varphi \uparrow_{\left(M_{i j}\right)_{+}}$and $\psi_{i j}:=\psi \uparrow_{\left(M_{i j}\right)_{+}}$for $i, j=1,2$. Then, $\varphi_{i j}$ and $\psi_{i j}$ are n.s.f. weights on $M_{i j}$.

- For all $i, j, k=1,2$, we denote by $\Delta_{i j}^{k}: M_{i j} \rightarrow M_{i k} \otimes M_{k j}$ the unital normal *homomorphism given by

$$
\Delta_{i j}^{k}(x):=\left(p_{i k} \otimes p_{k j}\right) \Delta(x), \quad \text { for all } x \in M_{i j} .
$$

- We have $J p_{k l}=p_{k l} J, \widehat{J} p_{k l}=p_{l k} \widehat{J}$ and $U p_{k l}=p_{l k} U$ for all $k, l=1,2$. We define the anti-unitaries $J_{k l}: \mathscr{H}_{k l} \rightarrow \mathscr{H}_{k l}, \widehat{J}_{k l}: \mathscr{H}_{k l} \rightarrow \mathscr{H}_{l k}$ and the unitary $U_{k l}: \mathscr{H}_{k l} \rightarrow \mathscr{H}_{l k}$ by setting $J_{k l}=p_{k l} J p_{k l}, \widehat{J}_{k l}=p_{l k} \widehat{J} p_{k l}$ and $U_{k l}=p_{l k} U p_{k l}=\widehat{J}_{k l} J_{k l}$.

- For $i, j, k, l=1,2$, let $\Sigma_{i j \otimes k l}:=\Sigma_{\mathscr{H}_{i j} \otimes \mathscr{H}_{k l}}: \mathscr{H}_{i j} \otimes \mathscr{H}_{k l} \rightarrow \mathscr{H}_{k l} \otimes \mathscr{H}_{i j}$.

We readily obtain:

$$
M=\bigoplus_{i, j=1,2} M_{i j} ; \quad \mathscr{H}=\bigoplus_{i, j=1,2} \mathscr{H}_{i j} ; \quad \Delta\left(p_{i j}\right)=p_{i 1} \otimes p_{1 j}+p_{i 2} \otimes p_{2 j}, \text { for all } i, j=1,2 .
$$

Note that in terms of the parts $\Delta_{i j}^{k}$ of $\Delta$, the coassociativity condition reads as follows:

$$
\left(\Delta_{i k}^{l} \otimes \operatorname{id}_{M_{k j}}\right) \Delta_{i j}^{k}=\left(\operatorname{id}_{M_{i l}} \otimes \Delta_{l j}^{k}\right) \Delta_{i j}^{l}, \quad \text { for all } i, j, k, l=1,2 .
$$

The G.N.S. representation for $\left(M_{i j}, \varphi_{i j}\right)$ is obtained by restriction of the G.N.S. representation of $(M, \varphi)$ to $M_{i j}$. In particular, the G.N.S. space $\mathscr{H}_{\varphi_{i j}}$ is identified with $\mathscr{H}_{i j}$.

3.3.4 Proposition. - For all $i, j, k, l=1,2$, we have:

$$
\begin{aligned}
\left(p_{i j} \otimes 1\right) V\left(p_{k l} \otimes 1\right) & =\delta_{k}^{i}\left(p_{i j} \otimes p_{j l}\right) V\left(p_{i l} \otimes p_{j l}\right) ; \\
\left(1 \otimes p_{i j}\right) W\left(1 \otimes p_{k l}\right) & =\delta_{j}^{l}\left(p_{i k} \otimes p_{i j}\right) W\left(p_{i k} \otimes p_{k j}\right) ; \\
\left(1 \otimes p_{j i}\right) \widetilde{V}\left(1 \otimes p_{l k}\right) & =\delta_{j}^{l}\left(p_{k i} \otimes p_{j i}\right) \widetilde{V}\left(p_{k i} \otimes p_{j k}\right) .
\end{aligned}
$$

3.3.5 Notations. - The operators $V, W$ and $\widetilde{V}$ each splits up into eight unitaries $V_{j l}^{i}: \mathscr{H}_{i l} \otimes \mathscr{H}_{j l} \rightarrow \mathscr{H}_{i j} \otimes \mathscr{H}_{j l}, W_{i k}^{j}: \mathscr{H}_{i k} \otimes \mathscr{H}_{k j} \rightarrow \mathscr{H}_{i k} \otimes \mathscr{H}_{i j}$ and $\widetilde{V}_{k i}^{j}: \mathscr{H}_{k i} \otimes \mathscr{H}_{j k} \rightarrow \mathscr{H}_{k i} \otimes \mathscr{H}_{j i}$ for $i, j, k, l=1,2$, given by $V_{j l}^{i}=\left(p_{i j} \otimes p_{j l}\right) V\left(p_{i l} \otimes p_{j l}\right), W_{i k}^{j}=\left(p_{i k} \otimes p_{i j}\right) W\left(p_{i k} \otimes p_{k j}\right)$ and $\widetilde{V}_{k i}^{j}=\left(p_{k i} \otimes p_{j i}\right) \widetilde{V}\left(p_{k i} \otimes p_{j k}\right)$.

Let $i, j, k, l, l^{\prime}=1,2$. These unitaries are related to each other by the following relations (cf. 3.1.3:

$$
\begin{gathered}
W_{i k}^{j}=\Sigma_{i j \otimes i k}\left(U_{j i} \otimes 1\right) V_{i k}^{j}\left(U_{j k}^{*} \otimes 1\right) \Sigma_{i k \otimes k j} ; \widetilde{V}_{k i}^{j}=\Sigma_{j i \otimes k i}\left(1 \otimes U_{i k}\right) V_{i k}^{j}\left(\otimes U_{i k}^{*}\right) \Sigma_{k i \otimes j k} ; \\
\widetilde{V}_{k i}^{j}=\left(U_{i k} \otimes U_{i j}\right) W_{i k}^{j}\left(U_{i k}^{*} \otimes U_{k j}^{*}\right) .
\end{gathered}
$$

Furthermore, we also have:

$$
\left(V_{j l}^{i}\right)^{*}=\left(J_{i l} \otimes \widehat{J}_{l j}\right) V_{l j}^{i}\left(J_{i j} \otimes \widehat{J}_{j l}\right) \quad \text { and } \quad\left(W_{i k}^{l}\right)^{*}=\left(\widehat{J}_{k i} \otimes J_{k j}\right) W_{k i}^{j}\left(\widehat{J}_{i k} \otimes J_{i j}\right) .
$$

Moreover, these unitaries satisfy the following pentagonal equations:

$$
\begin{aligned}
\left(V_{j k}^{i}\right)_{12}\left(V_{k l}^{i}\right)_{13}\left(V_{k l}^{j}\right)_{23}= & \left(V_{k l}^{j}\right)_{23}\left(V_{j l}^{i}\right)_{12} ; \quad\left(W_{i j}^{k}\right)_{12}\left(W_{i j}^{l}\right)_{13}\left(W_{j k}^{l}\right)_{23}=\left(W_{i k}^{l}\right)_{23}\left(W_{i j}^{k}\right)_{12} ; \\
& \left(\widetilde{V}_{j i}^{k}\right)_{12}\left(\widetilde{V}_{j i}^{l}\right)_{13}\left(\widetilde{V}_{k j}^{l}\right)_{23}=\left(\widetilde{V}_{k i}^{l}\right)_{23}\left(\widetilde{V}_{j i}^{k}\right)_{12} .
\end{aligned}
$$


We also have the following commutation relations:

$$
\left(V_{k j}^{l}\right)_{23}\left(W_{l l^{\prime}}^{j}\right)_{12}=\left(W_{l l^{\prime}}^{k}\right)_{12}\left(V_{k j}^{l^{\prime}}\right)_{23} ; \quad\left(V_{k i}^{l}\right)_{12}\left(\widetilde{V}_{k i}^{j}\right)_{23}=\left(\widetilde{V}_{k i}^{j}\right)_{23}\left(V_{k i}^{l}\right)_{12} .
$$

Furthermore, we have

$$
\Delta_{i j}^{k}(x)=\left(W_{i k}^{j}\right)^{*}(1 \otimes x) W_{i k}^{j}=V_{k j}^{i}(x \otimes 1)\left(V_{k j}^{i}\right)^{*}, \quad \text { for all } x \in M_{i j} .
$$

Note that for all $\omega \in \mathcal{B}(\mathscr{H})_{*}$ we have:

$$
\begin{aligned}
\left(\mathrm{id} \otimes p_{j l} \omega p_{j l}\right)\left(V_{j l}^{i}\right)= & p_{i j}(\mathrm{id} \otimes \omega)(V) p_{i l} ; \quad\left(p_{i k} \omega p_{i k} \otimes \mathrm{id}\right)\left(W_{i k}^{j}\right)=p_{i j}(\omega \otimes \mathrm{id})(W) p_{k j} ; \\
& \left(p_{k i} \omega p_{k i} \otimes \mathrm{id}\right)\left(\widetilde{V}_{k i}^{j}\right)=p_{j i}(\mathrm{id} \otimes \omega)(\widetilde{V}) p_{j k} .
\end{aligned}
$$

3.3.6 Proposition. - Let $i, j=1,2$ such that $i \neq j$. With the notations of 3.3.3. we have:

1. $\mathbb{G}_{i}:=\left(M_{i i}, \Delta_{i i}^{i}, \varphi_{i i}, \psi_{i i}\right)$ is a locally compact quantum group whose left (resp. right) regular representation is $W_{i i}^{i}\left(\right.$ resp. $\left.V_{i i}^{i}\right)$;

2. $\left(M_{i j}, \Delta_{i j}^{j}\right)$ is a right Galois object for $\mathbb{G}_{j}$ whose canonical implementation is $V_{j j}^{i}$;

3. $\left(M_{i j}, \Delta_{i j}^{i}\right)$ is a left Galois object for $\mathbb{G}_{i}$ whose canonical implementation is $W_{i i}^{j}$;

4. the actions $\Delta_{i j}^{j}$ and $\Delta_{i j}^{i}$ on $M_{i j}$ commute;

5. the Galois isometry associated with the right Galois object $\left(M_{i j}, \Delta_{i j}^{j}\right)$ for $\mathbb{G}_{j}(c f .6 .4 .1,6.4 .2$ [12]) is the unitary $\Sigma_{i j \otimes j j}\left(W_{i j}^{j}\right)^{*} \Sigma_{i j \otimes i j}$.

3.3.7 Definition. - A measured quantum groupoid $\left(\mathbb{C}^{2}, M, \alpha, \beta, \Delta, T, T^{\prime}, \epsilon\right)$ such that the source and target maps have range in $\mathcal{Z}(M)$ and generate a copy of $\mathbb{C}^{4}$ will be denoted by $\mathcal{G}_{\mathrm{G}_{1}, \mathrm{G}_{2}}$, where $\mathbb{G}_{i}=\left(M_{i i}, \Delta_{i i}^{i}, \varphi_{i i}, \psi_{i i}\right)$ (cf. 3.3.6) and will be called a colinking measured quantum groupoid.

3.3.8 Definition. - Let $G$ and $\mathbb{H}$ be two locally compact quantum groups. We say that $\mathbb{G}$ and $\mathrm{H}$ are monoidally equivalent if there exists a colinking measured quantum groupoid $\mathcal{G}_{\mathrm{G}_{1}, \mathrm{G}_{2}}$ between two locally compact quantum groups $\mathrm{G}_{1}$ and $\mathrm{G}_{2}$ such that $\mathbb{H}$ (resp. $\mathrm{G}$ ) is isomorphic to $\mathrm{G}_{1}$ (resp. $\mathrm{G}_{2}$ ).

Let $(S, \delta)$ be the weak Hopf $C^{*}$-algebra associated with $\mathcal{G}$. Note that

$$
p_{i j}=\alpha\left(\varepsilon_{i}\right) \beta\left(\varepsilon_{j}\right) \in \mathcal{Z}(\mathcal{M}(S)), \quad \text { for all } i, j=1,2 \text {. }
$$

3.3.9 Notations. - Let us recall the notations below (cf. 2.26 [2]).

1. Let $S_{i j}:=p_{i j} S$, for $i, j=1,2$. Then, $S_{i j}$ is a $C^{*}$-algebra (actually a closed two-sided ideal) of $S$ weakly dense in $M_{i j}$.

2. For $i, j, k=1,2$, let $\iota_{i j}^{k}: \mathcal{M}\left(S_{i k} \otimes S_{k j}\right) \rightarrow \mathcal{M}(S \otimes S)$ be the unique strictly continuous extension of the inclusion map $S_{i k} \otimes S_{k j} \subset S \otimes S$ satisfying $\iota_{i j}^{k}\left(1_{S_{i k} \otimes S_{k j}}\right)=p_{i k} \otimes p_{k j}$.

3. Let $\delta_{i j}^{k}: S_{i j} \rightarrow \mathcal{M}\left(S_{i k} \otimes S_{k j}\right)$ be the unique ${ }^{*}$-homomorphism such that

$$
\iota_{i j}^{k} \circ \delta_{i j}^{k}(x)=\left(p_{i k} \otimes p_{k j}\right) \delta(x), \quad \text { for all } x \in S_{i j} .
$$

With these notations, we have:

3.3.1o Proposition. - (cf. 7.4.13, 7.4.14 [12], 2.27 [2]) Let $i, j, k, l=1,2$.

1. $\left(\delta_{i k}^{l} \otimes \mathrm{id}_{S_{k j}}\right) \delta_{i j}^{k}=\left(\mathrm{id}_{S_{i l}} \otimes \delta_{l j}^{k}\right) \delta_{i j}^{l}$.

2. $\delta_{i j}^{k}(x)=\left(W_{i k}^{j}\right)^{*}\left(1_{\mathscr{H}_{i k}} \otimes x\right) W_{i k}^{j}=V_{k j}^{i}\left(x \otimes 1_{\mathscr{H}_{k j}}\right)\left(V_{k j}^{i}\right)^{*}$, for all $x \in S_{i j}$.

3. $\left[\delta_{i j}^{k}\left(S_{i j}\right)\left(1_{S_{i k}} \otimes S_{k j}\right)\right]=S_{i k} \otimes S_{k j}=\left[\delta_{i j}^{k}\left(S_{i j}\right)\left(S_{i k} \otimes 1_{S_{k j}}\right)\right]$. In particular, we have

$$
S_{k j}=\left[\left(\operatorname{id}_{S_{i k}} \otimes \omega\right) \delta_{i j}^{k}(x) ; x \in S_{i j}, \omega \in \mathcal{B}\left(\mathscr{H}_{k j}\right)_{*}\right] .
$$

4. The pair $\left(S_{j j}, \delta_{j j}^{j}\right)$ is the Hopf $C^{*}$-algebra associated with $\mathbf{G}_{j}$. 
The notion of regular measured quantum groupoid has been introduced in [16 and studied in the compact case. Note that this notion has been generalized in the setting of pseudo-multiplicative unitaries, cf. [23, 24]. The notion of semi-regular measured quantum groupoid has been introduced in [2, 9], where the notions of regularity and semi-regularity have been studied in the case of a finite-dimensional basis.

In this chapter, we fix a measured quantum groupoid $\mathcal{G}=\left(N, M, \alpha, \beta, \Delta, T, T^{\prime}, \epsilon\right)$ on the finite-dimensional basis $N=\bigoplus_{1 \leqslant l \leqslant k} \mathrm{M}_{n_{l}}(\mathbb{C})$ and we use all the notations introduced in $\$ \leqslant 3.1$. 3.2. In the appendix (cf. 8.2.1), for any $\xi \in \mathscr{H}$ we have given the definition of the operator

$$
R_{\tilde{\zeta}}^{\alpha} \in \mathcal{B}\left(\mathscr{H}_{\epsilon}, \mathscr{H}\right) \quad\left(\text { resp. } L_{\xi}^{\beta} \in \mathcal{B}\left(\mathscr{H}_{\epsilon}, \mathscr{H}\right)\right)
$$

and the definition of the weakly dense ideal of $\alpha(N)^{\prime}\left(\right.$ resp. $\left.\beta\left(N^{\mathrm{o}}\right)^{\prime}\right)$

$$
\mathcal{K}_{\alpha}:=\left[R_{\tilde{\xi}}^{\alpha}\left(R_{\eta}^{\alpha}\right)^{*} ; \xi, \eta \in \mathscr{H}\right] \quad\left(\text { resp. } \mathcal{K}_{\beta}:=\left[L_{\xi}^{\beta}\left(L_{\eta}^{\beta}\right)^{*} ; \xi, \eta \in \mathscr{H}\right]\right) .
$$

Note that $\mathcal{K}_{\alpha}$ and $\mathcal{K}_{\beta}$ are $C^{*}$-subalgebras of $\mathcal{K}:=\mathcal{K}(\mathscr{H})$.

We first recall the following important consequence of the irreducibility (cf. 2.13 [2]) of $\mathcal{G}$.

4.1 Proposition. - (cf. 2.15 [2]) The Banach spaces $[S \widehat{S}]$ and $\mathcal{C}(V)$ (cf. 2.1.2. are $C^{*}$-algebras and we have $[S \widehat{S}]=U \mathcal{C}(V) U^{*}$.

4.2 Definition. - (cf. 4.7 [16], 2.37 [2]) The groupoid $\mathcal{G}$ is said to be semi-regular (resp. regular) if we have $\mathcal{K}_{\beta} \subset \mathcal{C}(V)$ (resp. $\mathcal{K}_{\beta}=\mathcal{C}(V)$ ).

4.3 Proposition. - (cf. 2.8 [2], 3.2.8 [9]) The following statements are equivalent:

(i) $\mathcal{G}$ is semi-regular (resp. regular), i.e. $\mathcal{K}_{\alpha} \subset \mathcal{C}(W)\left(\right.$ resp. $\mathcal{K}_{\alpha}=\mathcal{C}(W)$ );

(ii) $\widehat{\mathcal{G}}$ is semi-regular (resp. regular), i.e. $\mathcal{K}_{\beta} \subset \mathcal{C}(V)$ (resp. $\mathcal{K}_{\beta}=\mathcal{C}(V)$ );

(iii) $\left(\mathcal{G}^{\mathrm{o}}\right)^{\mathrm{c}}$ is semi-regular (resp. regular), i.e. $\mathcal{K}_{\widehat{\alpha}} \subset \mathcal{C}(\widetilde{V})$ (resp. $\mathcal{K}_{\widehat{\alpha}}=\mathcal{C}(\widetilde{V})$ ).

4.4 Proposition. - (cf. 2.8 [2], 3.2.9 [9]) The following statements are equivalent:

(i) $\mathcal{G}$ is semi-regular (resp. regular);

(ii) $\mathcal{K}_{\widehat{\beta}} \subset[\widehat{S} S]\left(\operatorname{resp} . \mathcal{K}_{\widehat{\beta}}=[\widehat{S} S]\right)$;

(iii) $\mathcal{K}_{\alpha} \subset[R(S) \widehat{S}]\left(\right.$ resp. $\left.\mathcal{K}_{\alpha}=[R(S) \widehat{S}]\right)$;

(iv) $\mathcal{K}_{\widehat{\alpha}} \subset[S \lambda(\widehat{S})]\left(\operatorname{resp} . \mathcal{K}_{\widehat{\alpha}}=[S \lambda(\widehat{S})]\right)$.

In particular, if $\mathcal{G}$ is regular we have $[\widehat{S} S] \subset \mathcal{K},[R(S) \widehat{S}] \subset \mathcal{K}$ and $[S \lambda(\widehat{S})] \subset \mathcal{K}$ (and also $\mathcal{C}(V) \subset \mathcal{K}, \mathcal{C}(W) \subset \mathcal{K}$ and $\mathcal{C}(\widetilde{V}) \subset \mathcal{K})$.

The semi-regularity and the regularity of colinking measured quantum groupoids have been treated in detail in $\$ 2.5[2]$.

4.5 TheOREM. - (cf. $2.45[2]$ ) Let $\mathcal{G}_{\mathbb{G}_{1}, \mathbb{G}_{2}}$ be a colinking measured quantum groupoid associated with two monoidally equivalent locally compact quantum groups $\mathbb{G}_{1}$ and $\mathbb{G}_{2}$. The groupoid $\mathcal{G}_{\mathrm{G}_{1}, \mathbb{G}_{2}}$ is semi-regular (resp. regular) if, and only if, $\mathbb{G}_{1}$ and $\mathbb{G}_{2}$ are semi-regular (resp. regular).

In the following, we use the multi-index notation introduced in the appendix 88 of this article (cf. 8.2.21 8.2.22 and 8.2.23) with $\gamma:=\alpha$ and $\pi:=\beta$.

4.6 Lemma. - For all $\xi, \eta \in \mathscr{H}$, we have

$$
R_{\tilde{\zeta}}^{\alpha}\left(R_{\eta}^{\alpha}\right)^{*}=\sum_{I \in \mathscr{I}} n_{I}^{-1} \cdot e_{I} \theta_{\tilde{\zeta}, \eta} e_{\bar{I}} \quad \text { and } \quad L_{\xi}^{\beta}\left(L_{\eta}^{\beta}\right)^{*}=\sum_{I \in \mathscr{I}} n_{I}^{-1} \cdot f_{I} \theta_{\tilde{\zeta}, \eta} f_{\bar{I}} .
$$


Proof. For $\eta, \zeta \in \mathscr{H}$, let $X_{\eta, \zeta} \in N$ be defined by $X_{\eta, \zeta}:=\sum_{I \in \mathscr{I}} n_{I}^{-1}\left\langle e_{I} \eta, \zeta\right\rangle \cdot \varepsilon_{I}$. For all $x \in N$ and $\eta \in \mathscr{H}$, we have $R_{\eta}^{\alpha} \Lambda_{\epsilon}(x)=\alpha(x) \eta=\sum_{I \in \mathscr{I}} x_{I} \cdot e_{I} \eta$. Let $\eta, \zeta \in \mathscr{H}$ and $I \in \mathscr{I}$. $\left\langle\left(R_{\eta}^{\alpha}\right)^{*} \zeta, \Lambda_{\epsilon}\left(\varepsilon_{I}\right)\right\rangle=\left\langle\zeta, e_{I} \eta\right\rangle$. By disjunction elimination, we prove that $\epsilon\left(\varepsilon_{J I}\right)=\delta_{\bar{I}}^{J} n_{I}$ for all $I, J \in \mathscr{I}$. On the other hand, we have

$$
\begin{aligned}
\left\langle\Lambda_{\epsilon}\left(X_{\eta, \zeta}\right), \Lambda_{\epsilon}\left(\varepsilon_{I}\right)\right\rangle & =\epsilon\left(X_{\eta, \zeta}^{*} \varepsilon_{I}\right) \\
& =\epsilon\left(\sum_{J \in \mathscr{I}} n_{J}^{-1} \overline{\left\langle e_{\bar{J}} \eta, \zeta\right\rangle} \cdot \varepsilon_{J I}\right) \\
& =\sum_{J \in \mathscr{I}} n_{J}^{-1}\left\langle\zeta, e_{\bar{J}} \eta\right\rangle \epsilon\left(\varepsilon_{J I}\right) \\
& =\left\langle\zeta, e_{I} \eta\right\rangle .
\end{aligned}
$$

Hence, $\left\langle\left(R_{\eta}^{\alpha}\right)^{*} \zeta, \Lambda_{\epsilon}\left(\varepsilon_{I}\right)\right\rangle=\left\langle\Lambda_{\epsilon}\left(X_{\eta, \zeta}\right), \Lambda_{\epsilon}\left(\varepsilon_{I}\right)\right\rangle$. Hence, $\left(R_{\eta}^{\alpha}\right)^{*} \zeta=\Lambda_{\epsilon}\left(X_{\eta, \zeta}\right)$ for all $\eta, \zeta \in \mathscr{H}$. Let $\xi, \eta \in \mathscr{H}$. For all $\zeta \in \mathscr{H}$, we have

$$
R_{\tilde{\xi}}^{\alpha}\left(R_{\eta}^{\alpha}\right)^{*} \zeta=R_{\tilde{\xi}}^{\alpha} \Lambda_{\epsilon}\left(X_{\eta, \zeta}\right)=\sum_{I \in \mathscr{I}}\left(X_{\eta, \zeta}\right)_{I} \cdot e_{I} \xi=\sum_{I \in \mathscr{I}} n_{I}^{-1}\left\langle\eta, e_{\bar{I}} \zeta\right\rangle \cdot e_{I} \xi=\sum_{I \in \mathscr{I}} n_{I}^{-1} \theta_{e_{I} \xi, e_{I} \eta}(\zeta) .
$$

Hence, $R_{\mathcal{\zeta}}^{\alpha}\left(R_{\eta}^{\alpha}\right)^{*}=\sum_{I \in \mathscr{I}} n_{I}^{-1} \cdot e_{I} \theta_{\xi, \eta} e_{\bar{I}}$. The second formula is proved in a similar way.

We refer to 8.2.15 and 8.2 .18 for the definition of the operators $q_{\alpha}, q_{\beta}$ and $q_{\widehat{\alpha}}$. The propositions $4.7,4.8$ and 4.9 below have to be compared with their corresponding statements in the quantum group case, (f. $3.2 \mathrm{~b}$ ), $3.6 \mathrm{~b}$ ) and $3.6 \mathrm{~d}$ ) [4].

4.7 Proposition. - The following statements are equivalent:

(i) $\mathcal{G}$ is regular (resp. semi-regular);

(ii) $[(\mathcal{K} \otimes 1) W(1 \otimes \mathcal{K})]=\left[(\mathcal{K} \otimes 1) q_{\alpha}(1 \otimes \mathcal{K})\right]\left(\right.$ resp. $\left.\supset\left[(\mathcal{K} \otimes 1) q_{\alpha}(1 \otimes \mathcal{K})\right]\right)$;

(iii) $[(\mathcal{K} \otimes 1) V(1 \otimes \mathcal{K})]=\left[(\mathcal{K} \otimes 1) q_{\beta}(1 \otimes \mathcal{K})\right]\left(\right.$ resp. $\left.\supset\left[(\mathcal{K} \otimes 1) q_{\beta}(1 \otimes \mathcal{K})\right]\right) ;$

(iv) $[(\mathcal{K} \otimes 1) \widetilde{V}(1 \otimes \mathcal{K})]=\left[(\mathcal{K} \otimes 1) q_{\widehat{\alpha}}(1 \otimes \mathcal{K})\right]\left(\right.$ resp. $\left.\supset\left[(\mathcal{K} \otimes 1) q_{\widehat{\alpha}}(1 \otimes \mathcal{K})\right]\right)$.

Proof. It is known that $\mathcal{G}$ is regular (resp. semi-regular) if, and only if, $\widehat{\mathcal{G}}$ is regular (resp. semi-regular). Therefore, it suffices to prove that (i) is equivalent to (ii). We have

$$
[(\mathcal{K} \otimes 1) W(1 \otimes \mathcal{K})]=\Sigma(\mathcal{C}(W) \otimes \mathcal{K}) \quad(\text { cf. } 3.1[4])
$$

$\left(\mathcal{C}(W)\right.$ is a $C^{*}$-algebra regardless of the regularity of $\mathcal{G}$ ). Note that $\mathcal{G}$ is regular (resp. semiregular) if, and only if, $\Sigma(\mathcal{C}(W) \otimes \mathcal{K})=\Sigma\left(\mathcal{K}_{\alpha} \otimes \mathcal{K}\right)\left(\right.$ resp. $\left.\Sigma(\mathcal{C}(W) \otimes \mathcal{K}) \supset \Sigma\left(\mathcal{K}_{\alpha} \otimes \mathcal{K}\right)\right)$. Let $\xi, \eta, \zeta, \chi \in \mathscr{H}$. We have $e_{I} \theta_{\xi, \eta} e_{\bar{I}} \otimes \theta_{\zeta, \chi}=\theta_{e_{I} \xi, e_{I} \eta} \otimes \theta_{\zeta, \chi}=\theta_{e_{I} \xi \otimes \zeta, e_{I} \eta \otimes \chi}$, for all $I \in \mathscr{I}$. Hence, $\Sigma\left(e_{I} \theta_{\xi, \eta} e_{\bar{I}} \otimes \theta_{\zeta, \chi}\right)=\theta_{\zeta \otimes e_{I} \xi, e_{I} \eta \otimes \chi}=\theta_{\zeta, e_{I} \eta} \otimes \theta_{e_{I} \xi, \chi}=\theta_{\zeta, \eta} e_{\bar{I}} \otimes e_{I} \theta_{\xi, \chi}$ for all $I \in \mathscr{I}$. By Lemma 4.6, we obtain

$$
\Sigma\left(R_{\xi}^{\alpha}\left(R_{\eta}^{\alpha}\right)^{*} \otimes \theta_{\zeta, \chi}\right)=\left(\theta_{\zeta, \eta} \otimes 1\right) q_{\alpha}\left(1 \otimes \theta_{\xi, \chi}\right) .
$$

Hence, $\Sigma\left(\mathcal{K}_{\alpha} \otimes \mathcal{K}\right)=\left[(\mathcal{K} \otimes 1) q_{\alpha}(1 \otimes \mathcal{K})\right]$ and the equivalence $((\mathrm{i}) \Leftrightarrow(\mathrm{ii}))$ is proved.

4.8 Proposition. - If $\mathcal{G}$ is regular (resp. semi-regular), we have:

1. $[(S \otimes 1) W(1 \otimes \mathcal{K})]=\left[(S \otimes 1) q_{\alpha}(1 \otimes \mathcal{K})\right]\left(\right.$ resp. $\left.\supset\left[(S \otimes 1) q_{\alpha}(1 \otimes \mathcal{K})\right]\right) ;$

2. $[(\mathcal{K} \otimes 1) V(1 \otimes S)]=\left[(\mathcal{K} \otimes 1) q_{\beta}(1 \otimes S)\right]\left(\right.$ resp. $\left.\supset\left[(\mathcal{K} \otimes 1) q_{\beta}(1 \otimes S)\right]\right)$;

3. $[(R(S) \otimes 1) \widetilde{V}(1 \otimes \mathcal{K})]=\left[(R(S) \otimes 1) q_{\widehat{\alpha}}(1 \otimes \mathcal{K})\right]$ (resp. $\left.\supset\left[(R(S) \otimes 1) q_{\widehat{\alpha}}(1 \otimes \mathcal{K})\right]\right)$. 
Proof. Assume that $\mathcal{G}$ is regular (resp. semi-regular). Let us prove the first statement. The others will be obtained by using similar arguments. Let $a, b \in \mathcal{K}, \omega \in \mathcal{B}(\mathscr{H})_{*}$ and $y=(\mathrm{id} \otimes a \omega)(W)$. We have

$$
\begin{aligned}
(y \otimes 1) W(1 \otimes b) & =(\mathrm{id} \otimes \omega \otimes \mathrm{id})\left(W_{12} W_{13}(1 \otimes a \otimes b)\right) \\
& =(\mathrm{id} \otimes \omega \otimes \mathrm{id})\left(W_{23} W_{12}\left(1 \otimes W^{*}(a \otimes b)\right)\right) .
\end{aligned}
$$

However, $W^{*}(\mathcal{K} \otimes \mathcal{K})=q_{\beta \alpha}(\mathcal{K} \otimes \mathcal{K})$. Moreover, since $\left[W, 1 \otimes \beta\left(n^{\circ}\right)\right]=0$ for all $n \in N$, we have $\left[W_{12}, q_{\beta \alpha, 23}\right]=0$. Hence, $W_{23} W_{12} q_{\beta \alpha, 23}=W_{23} W_{12}$. We obtain (cf. $4 \cdot 7$ )

$$
\begin{aligned}
{[(S \otimes 1) W(1 \otimes \mathcal{K})] } & =\left[(\mathrm{id} \otimes a \omega \otimes \mathrm{id})\left(W_{23} W_{12}(1 \otimes 1 \otimes b)\right) ; \omega \in \mathcal{B}(\mathscr{H})_{*}, a, b \in \mathcal{K}\right] \\
& =\left[(\mathrm{id} \otimes \omega a \otimes \mathrm{id})\left(W_{23} W_{12}(1 \otimes 1 \otimes b)\right) ; \omega \in \mathcal{B}(\mathscr{H})_{*}, a, b \in \mathcal{K}\right] \\
& =\left[(\mathrm{id} \otimes \omega \otimes \mathrm{id})\left(((a \otimes 1) W(1 \otimes b))_{23} W_{12}\right) ; \omega \in \mathcal{B}(\mathscr{H})_{*}, a, b \in \mathcal{K}\right] \\
& =\left[(\mathrm{id} \otimes \omega \otimes \mathrm{id})\left(\left((a \otimes 1) q_{\alpha}(1 \otimes b)\right)_{23} W_{12}\right) ; \omega \in \mathcal{B}(\mathscr{H})_{*}, a, b \in \mathcal{K}\right] \\
\text { (resp. } & \left.\supset\left[(\mathrm{id} \otimes \omega \otimes \mathrm{id})\left(\left((a \otimes 1) q_{\alpha}(1 \otimes b)\right)_{23} W_{12}\right) ; \omega \in \mathcal{B}(\mathscr{H})_{*}, a, b \in \mathcal{K}\right]\right) .
\end{aligned}
$$

However, for all $\omega \in \mathcal{B}(\mathscr{H})_{*}$ and $a, b \in \mathcal{K}$ we have

$(\mathrm{id} \otimes \omega \otimes \mathrm{id})\left(\left((a \otimes 1) q_{\alpha}(1 \otimes b)\right)_{23} W_{12}\right)=(\mathrm{id} \otimes \omega a \otimes \mathrm{id})\left(q_{\alpha, 23} W_{12}(1 \otimes 1 \otimes b)\right)$.

Since $(1 \otimes \alpha(n)) W=W(\alpha(n) \otimes 1)$ for all $n \in N$, we have $q_{\alpha, 23} W_{12}=W_{12} q_{\alpha, 13}$. Hence,

$$
(\mathrm{id} \otimes \omega \otimes \mathrm{id})\left(\left((a \otimes 1) q_{\alpha}(1 \otimes b)\right)_{23} W_{12}\right)=((\mathrm{id} \otimes \omega a)(W) \otimes 1) q_{\alpha}(1 \otimes b)
$$

and the result is proved.

4.9 Proposition. - If $\mathcal{G}$ is regular (resp. semi-regular), then we have:

1. $[(S \otimes 1) W(1 \otimes \lambda(\widehat{S}))]=\left[(S \otimes 1) q_{\alpha}(1 \otimes \lambda(\widehat{S}))\right]\left(\right.$ resp. $\left.\supset\left[(S \otimes 1) q_{\alpha}(1 \otimes \lambda(\widehat{S}))\right]\right) ;$

2. $[(\widehat{S} \otimes 1) V(1 \otimes S)]=\left[(\widehat{S} \otimes 1) q_{\beta}(1 \otimes S)\right]\left(\right.$ resp. $\left.\supset\left[(\widehat{S} \otimes 1) q_{\beta}(1 \otimes S)\right]\right)$;

3. $[(R(S) \otimes 1) \widetilde{V}(1 \otimes \widehat{S})]=\left[(R(S) \otimes 1) q_{\widehat{\alpha}}(1 \otimes \widehat{S})\right]$ (resp. $\left.\supset\left[(R(S) \otimes 1) q_{\widehat{\alpha}}(1 \otimes \widehat{S})\right]\right)$.

In particular, we have $[(S \otimes 1) W(1 \otimes \lambda(\widehat{S}))] \subset S \otimes \lambda(\widehat{S}),[(\widehat{S} \otimes 1) V(1 \otimes S)] \subset \widehat{S} \otimes S$ and $[(R(S) \otimes 1) \widetilde{V}(1 \otimes \widehat{S})] \subset R(S) \otimes \widehat{S}$.

Proof. We have the pentagonal equation $V_{12} V_{13}=V_{23} V_{12} V_{23}^{*}$. Since $V \in \mathcal{M}(\widehat{S} \otimes S)$ is a partial isometry, we have $V^{*}(\widehat{S} \otimes S)=q_{\widehat{\alpha} \beta}(\widehat{S} \otimes S)$. Since $[V, 1 \otimes \widehat{\alpha}(n)]=0$ for all $n \in N$, we have $\left[V_{12}, q_{\widehat{\alpha} \beta, 23}\right]=0$. Hence, $V_{23} V_{12} q_{\widehat{\alpha} \beta, 23}=V_{23} V_{12}$. Hence,

$$
\begin{aligned}
{[(\widehat{S} \otimes 1) V(1 \otimes S)] } & =\left[((\mathrm{id} \otimes \omega)(V) \otimes 1) V(1 \otimes y) ; \omega \in \mathcal{B}(\mathscr{H})_{*}, y \in S\right] \\
& =\left[(\mathrm{id} \otimes \omega \otimes \mathrm{id})\left(V_{12} V_{13}(1 \otimes 1 \otimes y)\right) ; \omega \in \mathcal{B}(\mathscr{H})_{*}, y \in S\right] \\
& =\left[(\mathrm{id} \otimes \omega \otimes \mathrm{id})\left(V_{23} V_{12} V_{23}^{*}(1 \otimes x \otimes y)\right) ; \omega \in \mathcal{B}(\mathscr{H})_{*}, y \in S, x \in \widehat{S}\right] \\
& =\left[(\mathrm{id} \otimes \omega \otimes \mathrm{id})\left(V_{23} V_{12}(1 \otimes x \otimes y)\right) ; \omega \in \mathcal{B}(\mathscr{H})_{*}, x \in \widehat{S}, y \in S\right] \\
& =\left[(\mathrm{id} \otimes \omega \otimes \mathrm{id})\left(V_{23}(1 \otimes 1 \otimes y) V_{12}\right) ; \omega \in \mathcal{B}(\mathscr{H})_{*}, y \in S\right] \\
& =\left[(\mathrm{id} \otimes \omega \otimes \mathrm{id})\left(((a \otimes 1) V(1 \otimes y))_{23} V_{12}\right) ; \omega \in \mathcal{B}(\mathscr{H})_{*}, a \in \mathcal{K}, y \in S\right] .
\end{aligned}
$$

Let $X:=\left[(\mathrm{id} \otimes \omega \otimes \mathrm{id})\left(\left((a \otimes 1) q_{\beta}(1 \otimes y)\right)_{23} V_{12}\right) ; \omega \in \mathcal{B}(\mathscr{H})_{*}, a \in \mathcal{K}, y \in S\right]$. Since $\mathcal{G}$ is regular (resp. semi-regular), it follows from 4.8 that

$$
[(\widehat{S} \otimes 1) V(1 \otimes S)]=X \quad(\text { resp. }[(\widehat{S} \otimes 1) V(1 \otimes S)] \supset X) .
$$

However, since $\left(1 \otimes \beta\left(n^{\mathrm{o}}\right)\right) V=V\left(\beta\left(n^{\mathrm{o}}\right) \otimes 1\right)$ for all $n \in N$, we have

$$
\begin{aligned}
X & =\left[(\mathrm{id} \otimes \omega a \otimes \mathrm{id})\left(q_{\beta, 23} V_{12}(1 \otimes 1 \otimes y)\right) ; \omega \in \mathcal{B}(\mathscr{H})_{*}, a \in \mathcal{K}, y \in S\right] \\
& =\left[(\mathrm{id} \otimes \omega \otimes \mathrm{id})\left(V_{12} q_{\beta, 13}(1 \otimes 1 \otimes y)\right) ; \omega \in \mathcal{B}(\mathscr{H})_{*}, y \in S\right] \\
& =\left[((\mathrm{id} \otimes \omega)(V) \otimes 1) q_{\beta}(1 \otimes y) ; \omega \in \mathcal{B}(\mathscr{H})_{*}, y \in S\right] \\
& =\left[(\widehat{S} \otimes 1) q_{\beta}(1 \otimes S)\right] .
\end{aligned}
$$


The second statement is proved and the third one follows by applying it to $\widehat{\mathcal{G}}$. We obtain the first statement by combining the third one with the formulas $W=\left(U^{*} \otimes U^{*}\right) \widetilde{V}(U \otimes U)$ and $\widehat{\alpha}=\operatorname{Ad}_{U} \circ \alpha$. Finally, the last statement follows from the inclusions $\beta\left(N^{\mathrm{o}}\right) \subset \mathcal{M}(S)$, $\widehat{\beta}\left(N^{\mathrm{o}}\right) \subset \mathcal{M}(\widehat{S}), \alpha(N) \subset \mathcal{M}(S)$ and $\widehat{\alpha}(N) \subset \mathcal{M}(\widehat{S})$.

In the result below, we refer again to 8.2.15 and 8.2 .18 for the definition of the operators $q_{\beta \widehat{\beta}}, q_{\widehat{\alpha} \alpha}$ and $q_{\widehat{\beta} \beta}$.

4.10 COROllary. - If $\mathcal{G}$ is regular (resp. semi-regular), then we have:

1. $[(1 \otimes \lambda(\widehat{S})) W(S \otimes 1)]=\left[(1 \otimes \lambda(\widehat{S})) q_{\beta \widehat{\beta}}(S \otimes 1)\right]\left(\operatorname{resp} . \supset\left[(1 \otimes \lambda(\widehat{S})) q_{\beta \widehat{\beta}}(S \otimes 1)\right]\right) ;$

2. $[(1 \otimes S) V(\widehat{S} \otimes 1)]=\left[(1 \otimes S) q_{\widehat{\alpha} \alpha}(\widehat{S} \otimes 1)\right]\left(\operatorname{resp} . \supset\left[(1 \otimes S) q_{\widehat{\alpha} \alpha}(\widehat{S} \otimes 1)\right]\right)$;

3. $[(1 \otimes \widehat{S}) \widetilde{V}(R(S) \otimes 1)]=\left[(1 \otimes \widehat{S}) q_{\widehat{\beta} \beta}(R(S) \otimes 1)\right]\left(\operatorname{resp} . \supset\left[(1 \otimes \widehat{S}) q_{\widehat{\beta} \beta}(R(S) \otimes 1)\right]\right)$.

If $\mathcal{G}$ is regular, then we have $[(1 \otimes \lambda(\widehat{S})) W(S \otimes 1)] \subset S \otimes \lambda(\widehat{S}),[(1 \otimes S) V(\widehat{S} \otimes 1)] \subset \widehat{S} \otimes S$ and $[(1 \otimes \widehat{S}) \widetilde{V}(R(S) \otimes 1)] \subset R(S) \otimes \widehat{S}$.

Proof. This is a direct consequence of Proposition 4.9 and the formulas $\widehat{\beta}=\operatorname{Ad}_{U} \circ \beta$, $\widehat{\alpha}=\operatorname{Ad}_{U} \circ \alpha, W=\Sigma(U \otimes 1) V\left(U^{*} \otimes 1\right) \Sigma$ and $\widetilde{V}=\Sigma(1 \otimes U) V\left(1 \otimes U^{*}\right) \Sigma$. The second statement follows from the inclusions $\beta\left(N^{\mathrm{o}}\right) \subset \mathcal{M}(S), \widehat{\beta}\left(N^{\mathrm{o}}\right) \subset \mathcal{M}(\widehat{S}), \alpha(N) \subset \mathcal{M}(S)$ and $\widehat{\alpha}(N) \subset \mathcal{M}(\widehat{S})$.

5 MEASURED QUANTUM GROUPOIDS ON A FINITE BASIS IN ACTION

\subsection{Continuous actions, crossed product and biduality}

In this section, we fix a measured quantum groupoid $\mathcal{G}=\left(N, M, \alpha, \beta, \Delta, T, T^{\prime}, \epsilon\right)$ on the finite-dimensional basis $N=\bigoplus_{1 \leqslant l \leqslant k} \mathrm{M}_{n_{l}}(\mathbb{C})$ and we use all the notations introduced in $\S \S 3.1$ 3.2 In the following, we recall the definitions, notations and results of $\$ \S 3.1,3.2 .1$, 3.2.2 and 3.3.1 [2] (see also [9] chapter 4).

\subsubsection{Notion of actions of measured quantum groupoids on a finite basis}

5.1.1 Lemma. - Let $A$ and $B$ be two $C^{*}$-algebras, $f: A \rightarrow \mathcal{M}(B) a^{*}$-homomorphism and $e \in \mathcal{M}(B)$. The following statements are equivalent:

(i) there exists an approximate unit $\left(u_{\lambda}\right)_{\lambda}$ of $A$ such that $f\left(u_{\lambda}\right) \rightarrow e$ with respect to the strict topology;

(ii) $f$ extends to a strictly continuous *-homomorphism $f: \mathcal{M}(A) \rightarrow \mathcal{M}(B)$, necessarily unique, such that $f\left(1_{A}\right)=e$;

(iii) $[f(A) B]=e B$.

In that case, $e$ is a self-adjoint projection, for all approximate unit $\left(v_{\mu}\right)_{\mu}$ of $A$ we have $f\left(v_{\mu}\right) \rightarrow e$ with respect to the strict topology and $[B f(A)]=B e$.

5.1.2 Definition. - An action of $\mathcal{G}$ on a $C^{*}$-algebra $A$ is a pair $\left(\beta_{A}, \delta_{A}\right)$ consisting of a non-degenerate *-homomorphism $\beta_{A}: N^{\mathrm{o}} \rightarrow \mathcal{M}(A)$ and a faithful *-homomorphism $\delta_{A}: A \rightarrow \mathcal{M}(A \otimes S)$ such that:

1. $\delta_{A}$ extends to a strictly continuous *-homomorphism $\delta_{A}: \mathcal{M}(A) \rightarrow \mathcal{M}(A \otimes S)$ such that $\delta_{A}\left(1_{A}\right)=q_{\beta_{A} \alpha}$ (cf. 8.2.19;

2. $\left(\delta_{A} \otimes \mathrm{id}_{S}\right) \delta_{A}=\left(\mathrm{id}_{A} \otimes \delta\right) \delta_{A}$;

3. $\delta_{A}\left(\beta_{A}\left(n^{\mathrm{o}}\right)\right)=q_{\beta_{A} \alpha}\left(1_{A} \otimes \beta\left(n^{\mathrm{o}}\right)\right)$, for all $n \in N$. 
We say that the action $\left(\beta_{A}, \delta_{A}\right)$ is strongly continuous if we have

$$
\left[\delta_{A}(A)\left(1_{A} \otimes S\right)\right]=q_{\beta_{A} \alpha}(A \otimes S) .
$$

If that case, we say that the triple $\left(A, \beta_{A}, \delta_{A}\right)$ is a $\mathcal{G}$ - $C^{*}$-algebra.

5.1.3 REMARKs. - - By 5.1.1 the condition 1 is equivalent to requiring that for some (and then any) approximate unit $\left(u_{\lambda}\right)$ of $A$, we have $\delta_{A}\left(u_{\lambda}\right) \rightarrow q_{\beta_{A} \alpha}$ with respect to the strict topology of $\mathcal{M}(A \otimes S)$. It is also equivalent to $\left[\delta_{A}(A)(A \otimes S)\right]=q_{\beta_{A} \alpha}(A \otimes S)$.

- Condition 1 implies that the *-homomorphisms $\delta_{A} \otimes \mathrm{id}_{S}$ and $\mathrm{id}_{A} \otimes \delta$ extend uniquely to strictly continuous *-homomorphisms from $\mathcal{M}(A \otimes S)$ to $\mathcal{M}(A \otimes S \otimes S)$ such that $\left(\delta_{A} \otimes \mathrm{id}_{S}\right)\left(1_{A \otimes S}\right)=q_{\beta_{A} \alpha, 12}$ and $\left(\mathrm{id}_{A} \otimes \delta\right)\left(1_{A \otimes S}\right)=q_{\beta \alpha, 23}$. In particular, condition 2 does make sense and we denote by $\delta_{A}^{2}:=\left(\delta_{A} \otimes \mathrm{id}_{S}\right) \delta_{A}: A \rightarrow \mathcal{M}(A \otimes S \otimes S)$ the iterated coaction map.

5.1.4 Examples. - Let us give two basic examples.

- $(S, \beta, \delta)$ is a $\mathcal{G}-C^{*}$-algebra.

- Let $\beta_{N^{\circ}}:=\operatorname{id}_{N^{\circ}}$. Let $\delta_{N^{\circ}}: N^{\mathrm{o}} \rightarrow \mathcal{M}\left(N^{\mathrm{o}} \otimes S\right)$ be the faithful unital *-homomorphism given by $\delta_{N^{o}}\left(n^{\mathrm{o}}\right):=q_{\beta_{N^{\mathrm{o}}} \alpha}\left(1_{N^{\mathrm{o}}} \otimes \beta\left(n^{\mathrm{o}}\right)\right)$ for all $n \in N$. Then, the pair $\left(\beta_{N^{\mathrm{o}}}, \delta_{N^{\mathrm{o}}}\right)$ is an action of $\mathcal{G}$ on $N^{\mathrm{O}}$ called the trivial action.

5.1.5 Proposition. - Let $\left(\delta_{A}, \beta_{A}\right)$ be an action of $\mathcal{G}$ on $A$. We have the following statements:

1. the iterated coaction map $\delta_{A}^{2}$ extends uniquely to a strictly continuous *-homomorphism $\delta_{A}^{2}: \mathcal{M}(A) \rightarrow \mathcal{M}(A \otimes S \otimes S)$ such that $\delta_{A}^{2}\left(1_{A}\right)=q_{\beta_{A} \alpha, 12} q_{\beta \alpha, 23} ;$ moreover, we have $\delta_{A}^{2}(m)=\left(\delta_{A} \otimes \mathrm{id}_{S}\right) \delta_{A}(m)=\left(\mathrm{id}_{A} \otimes \delta\right) \delta_{A}(m)$ for all $m \in \mathcal{M}(A)$;

2. for all $n \in N$, we have $\delta_{A}\left(\beta_{A}\left(n^{\mathrm{o}}\right)\right)=\left(1_{A} \otimes \beta\left(n^{\mathrm{o}}\right)\right) q_{\beta_{A} \alpha}$;

3. if $\left(\beta_{A}, \delta_{A}\right)$ is strongly continuous, then we have $\left[\left(1_{A} \otimes S\right) \delta_{A}(A)\right]=(A \otimes S) q_{\beta_{A} \alpha}$.

Let us provide a more explicit definition of what an action of the dual measured quantum groupoid $\widehat{\mathcal{G}}$ on a $C^{*}$-algebra $B$ is.

5.1.6 Definition. - An action of $\widehat{\mathcal{G}}$ on a $C^{*}$-algebra $B$ is a pair $\left(\alpha_{B}, \delta_{B}\right)$ consisting of a non-degenerate ${ }^{*}$-homomorphism $\alpha_{B}: N \rightarrow \mathcal{M}(B)$ and a faithful ${ }^{*}$-homomorphism $\delta_{B}: B \rightarrow \mathcal{M}(B \otimes \widehat{S})$ such that:

1. $\delta_{B}$ extends to a strictly continuous *-homomorphism $\delta_{B}: \mathcal{M}(B) \rightarrow \mathcal{M}(B \otimes \widehat{S})$ such that $\delta_{B}\left(1_{B}\right)=q_{\alpha_{B} \beta}$ (cf. 8.2.19);

2. $\left(\delta_{B} \otimes \mathrm{id}_{\widehat{S}}\right) \delta_{B}=\left(\mathrm{id}_{B} \otimes \widehat{\delta}\right) \delta_{B}$;

3. $\delta_{B}\left(\alpha_{B}(n)\right)=q_{\alpha_{B} \beta}\left(1_{B} \otimes \widehat{\alpha}(n)\right)$, for all $n \in N$.

We say that the action $\left(\alpha_{B}, \delta_{B}\right)$ is strongly continuous if we have

$$
\left[\delta_{B}(B)\left(1_{B} \otimes \widehat{S}\right)\right]=q_{\alpha_{B} \beta}(B \otimes \widehat{S}) .
$$

If $\left(\delta_{B}, \alpha_{B}\right)$ is a strongly continuous action of $\widehat{\mathcal{G}}$ on $B$, we say that the triple $\left(B, \alpha_{B}, \delta_{B}\right)$ is a $\widehat{\mathcal{G}}$ - $C^{*}$-algebra.

5.1.7 REMARKs. - As for actions of $\mathcal{G}$, we have:

- the condition 1 is equivalent to requiring that for some (and then any) approximate unit $\left(u_{\lambda}\right)_{\lambda}$ of $B$ we have $\delta_{B}\left(u_{\lambda}\right) \rightarrow q_{\alpha_{B} \beta}$ with respect to the strict topology, which is also equivalent to the relation $\left[\delta_{B}(B)(B \otimes \widehat{S})\right]=q_{\alpha_{B} \beta}(B \otimes \widehat{S})$;

- the *-homomorphisms $\operatorname{id}_{B} \otimes \widehat{\delta}$ and $\delta_{B} \otimes \mathrm{id}_{\widehat{S}}$ extend uniquely to strictly continuous *homomorphisms from $\mathcal{M}(B \otimes \widehat{S})$ to $\mathcal{M}(B \otimes \widehat{S} \otimes \widehat{S})$ such that $\left(\operatorname{id}_{B} \otimes \widehat{\delta}\right)\left(1_{B \otimes \widehat{S}}\right)=q_{\widehat{\alpha} \beta, 23}$ and $\left(\delta_{B} \otimes \mathrm{id}_{\widehat{S}}\right)\left(1_{B \otimes \widehat{S}}\right)=q_{\alpha_{B} \beta, 12}$. In particular, condition 2 does make sense and we denote by $\delta_{B}^{2}:=\left(\delta_{B} \otimes \mathrm{id}_{\widehat{S}}\right) \delta_{B}: B \rightarrow \mathcal{M}(B \otimes \widehat{S} \otimes \widehat{S})$ the iterated coaction map. 
5.1.8 ExAMPLES. - Let us give two basic examples:

- $(\widehat{S}, \widehat{\alpha}, \widehat{\delta})$ is a $\widehat{\mathcal{G}}$ - $\mathrm{C}^{*}$-algebra;

- Let $\alpha_{N}:=\operatorname{id}_{N}$ and $\delta_{N}: N \rightarrow \mathcal{M}(N \otimes \widehat{S}) ; n \mapsto q_{\alpha_{N} \beta}\left(1_{N} \otimes \widehat{\alpha}(n)\right)$; then, the pair $\left(\alpha_{N}, \delta_{N}\right)$ is an action of $\widehat{\mathcal{G}}$ on $N$ called the trivial action.

5.1.9 Proposition. - Let $\left(\alpha_{B}, \delta_{B}\right)$ be an action of $\widehat{\mathcal{G}}$ on $B$. We have the following statements:

1. the iterated coaction map $\delta_{B}^{2}$ extends uniquely to a strictly continuous *-homomorphism $\delta_{B}^{2}: \mathcal{M}(B) \rightarrow \mathcal{M}(B \otimes \widehat{S} \otimes \widehat{S})$ such that $\delta_{B}^{2}\left(1_{B}\right)=q_{\alpha_{B} \beta, 12} q_{\widehat{\alpha} \beta, 23} ;$ moreover, we have $\delta_{B}^{2}(m)=\left(\delta_{B} \otimes \mathrm{id}_{\widehat{S}}\right) \delta_{B}(m)=\left(\mathrm{id}_{B} \otimes \widehat{\delta}\right) \delta_{B}(m)$ for all $m \in \mathcal{M}(B)$;

2. for all $n \in N$, we have $\delta_{B}\left(\alpha_{B}(n)\right)=\left(1_{B} \otimes \widehat{\alpha}(n)\right) q_{\alpha_{B} \beta}$;

3. if $\left(\alpha_{B}, \delta_{B}\right)$ is strongly continuous, then we have $\left[\left(1_{B} \otimes \widehat{S}\right) \delta_{B}(B)\right]=(B \otimes \widehat{S}) q_{\alpha_{B} \beta}$.

5.1.1o Definition. - For $i=1,2$, let $A_{i}$ (resp. $B_{i}$ ) be a $C^{*}$-algebra. For $i=1,2$, let $\left(\beta_{A_{i}}, \delta_{A_{i}}\right)$ (resp. $\left.\left(\alpha_{B_{i}}, \delta_{B_{i}}\right)\right)$ be an action of $\mathcal{G}$ (resp. $\widehat{\mathcal{G}}$ ) on $A_{i}$ (resp. $\left.B_{i}\right)$. A non-degenerate ${ }^{*}$ homomorphism $f: A_{1} \rightarrow \mathcal{M}\left(A_{2}\right)$ (resp. $f: B_{1} \rightarrow \mathcal{M}\left(B_{2}\right)$ ) is said to be $\mathcal{G}$-equivariant (resp. $\widehat{\mathcal{G}}$-equivariant) if $\left(f \otimes \mathrm{id}_{S}\right) \delta_{A_{1}}=\delta_{A_{2}} \circ f$ and $f \circ \beta_{A_{1}}=\beta_{A_{2}}\left(\right.$ resp. $\left(f \otimes \mathrm{id}_{\widehat{S}}\right) \delta_{B_{1}}=\delta_{B_{2}} \circ f$ and $\left.f \circ \alpha_{B_{1}}=\alpha_{B_{2}}\right)$.

5.1.11 REMARK. - With the notations and hypotheses of 5.1.10, if $f$ satisfies the relation $\left(f \otimes \mathrm{id}_{S}\right) \delta_{A_{1}}=\delta_{A_{2}} \circ f\left(\mathrm{resp} .\left(f \otimes \mathrm{id}_{\widehat{S}}\right) \delta_{B_{1}}=\delta_{B_{2}} \circ f\right)$, then $f$ satisfies necessarily the relation $f \circ \beta_{A_{1}}=\beta_{A_{2}}$ (resp. $f \circ \alpha_{B_{1}}=\alpha_{B_{2}}$ ), i.e. $f$ is $\mathcal{G}$-equivariant (resp. $\widehat{\mathcal{G}}$-equivariant). Indeed, let $n \in N$. For all $a \in A_{1}$ and $x \in A_{2}$, we have

$$
\begin{aligned}
\delta_{A_{2}}\left(f\left(\beta_{A_{1}}\left(n^{\mathrm{o}}\right)\right) f(a) x\right) & =\left(f \otimes \mathrm{id}_{S}\right) \delta_{A_{1}}\left(\beta_{A_{1}}\left(n^{\mathrm{o}}\right) a\right) \delta_{A_{2}}(x) \\
& =\left(1_{A_{2}} \otimes \beta\left(n^{\mathrm{o}}\right)\right)\left(f \otimes \mathrm{id}_{S}\right) \delta_{A_{1}}(a) \delta_{A_{2}}(x) \\
& =\left(1_{A_{2}} \otimes \beta\left(n^{\mathrm{o}}\right)\right) \delta_{A_{2}}(f(a) x) \\
& =\delta_{A_{2}}\left(\beta_{A_{2}}\left(n^{\mathrm{o}}\right) f(a) x\right) .
\end{aligned}
$$

Hence, $f\left(\beta_{A_{1}}\left(n^{\mathrm{o}}\right)\right) f(a) x=\beta_{A_{2}}\left(n^{\mathrm{o}}\right) f(a) x$ for all $a \in A_{1}$ and $x \in A_{2}$ since $\delta_{A_{2}}$ is faithful. Hence, we have $f\left(\beta_{A_{1}}\left(n^{\mathrm{o}}\right)\right)=\beta_{A_{2}}\left(n^{\mathrm{o}}\right)$ since $f$ is non-degenerate.

5.1.12 Notation. - We denote by $\mathrm{Alg}_{\mathcal{G}}$ the category whose objects are the $\mathcal{G}$ - $\mathrm{C}^{*}$-algebras and whose set of arrows between $\mathcal{G}$ - $\mathrm{C}^{*}$-algebras is the set of $\mathcal{G}$-equivariant non-degenerate *-homomorphisms.

\subsubsection{Crossed product and dual action}

Let us fix a strongly continuous action $\left(\beta_{A}, \delta_{A}\right)$ of $\mathcal{G}$ on a $C^{*}$-algebra $A$.

5.1.13 Notations. - The *-representation

$$
\pi_{L}:=\left(\mathrm{id}_{A} \otimes L\right) \circ \delta_{A}: A \rightarrow \mathcal{L}(A \otimes \mathscr{H})
$$

of $A$ on the Hilbert $A$-module $A \otimes \mathscr{H}$ extends uniquely to a strictly/*-strongly continuous faithful *-representation $\pi_{L}: \mathcal{M}(A) \rightarrow \mathcal{L}(A \otimes \mathscr{H})$ such that $\pi_{L}\left(1_{A}\right)=q_{\beta_{A} \alpha}$. Moreover, we have $\pi_{L}(m)=\pi_{L}(m) q_{\beta_{A} \alpha}=q_{\beta_{A} \alpha} \pi_{L}(m)$ for all $m \in \mathcal{M}(A)$. Consider the Hilbert $A$-module

$$
\mathcal{E}_{A, L}:=q_{\beta_{A} \alpha}(A \otimes \mathscr{H}) .
$$

By restricting $\pi_{L}$, we obtain a strictly ${ }^{*}$-strongly continuous faithful unital *-representation

$$
\pi: \mathcal{M}(A) \rightarrow \mathcal{L}\left(\mathcal{E}_{A, L}\right) ; m \mapsto \pi_{L}(m)\left\lceil_{\mathcal{E}_{A, L}}\right.
$$

We have $\left[1_{A} \otimes T, q_{\beta_{A} \alpha}\right]=0$ for all $T \in \mathcal{M}(\widehat{S})$. We then obtain a strictly $/^{*}$-strongly continuous unital *-representation

$$
\widehat{\theta}: \mathcal{M}(\widehat{S}) \rightarrow \mathcal{L}\left(\mathcal{E}_{A, L}\right) ; T \mapsto\left(1_{A} \otimes T\right)\left\lceil_{\mathcal{E}_{A, L}} .\right.
$$

Note that if $\beta_{A}$ is faithful, then so is $\widehat{\theta}$. 
5.1.14 Proposition-Definition. - The norm closed subspace of $\mathcal{L}\left(\mathcal{E}_{A, L}\right)$ spanned by the products of the form $\pi(a) \hat{\theta}(x)$ for $a \in A$ and $x \in \widehat{S}$ is $a C^{*}$-subalgebra called the (reduced) crossed product of $A$ by the strongly continuous action $\left(\beta_{A}, \delta_{A}\right)$ of $\mathcal{G}$ and denoted by $A \rtimes \mathcal{G}$.

In particular, the morphism $\pi$ (resp. $\widehat{\theta}$ ) defines a faithful unital *-homomorphism (resp. unital *-homomorphism) $\pi: \mathcal{M}(A) \rightarrow \mathcal{M}(A \rtimes \mathcal{G})($ resp. $\widehat{\theta}: \widehat{S} \rightarrow \mathcal{M}(A \rtimes \mathcal{G}))$.

Since $[\widetilde{V}, \alpha(n) \otimes 1]=0$, we have $\left[\widetilde{V}_{23}, q_{\beta_{A} \alpha, 12}\right]=0$. The operator $\widetilde{V}_{23} \in \mathcal{L}(A \otimes \mathscr{H} \otimes \mathscr{H})$ restricts to a partial isometry

$$
X:=\widetilde{V}_{23} \uparrow_{\mathcal{E}_{A, L} \otimes \mathscr{H}} \in \mathcal{L}\left(\mathcal{E}_{A, L} \otimes \mathscr{H}\right),
$$

whose initial and final projections are $X^{*} X=\left.q_{\widehat{\beta} \alpha, 23}\right|_{\mathcal{E}_{A, L} \otimes \mathscr{H}}$ and $X X^{*}=\left.q_{\widehat{\alpha} \beta, 23}\right|_{\mathcal{E}_{A, L} \otimes \mathscr{H}}$.

5.1.15 Proposition-Definition. - Let

$$
\delta_{A \rtimes \mathcal{G}}: A \rtimes \mathcal{G} \rightarrow \mathcal{L}\left(\mathcal{E}_{A, L} \otimes \mathscr{H}\right) \quad \text { and } \quad \alpha_{A \rtimes \mathcal{G}}: N \rightarrow \mathcal{M}(A \rtimes \mathcal{G})
$$

be the linear maps defined by:

- $\delta_{A \rtimes \mathcal{G}}(b):=X(b \otimes 1) X^{*}$, for all $b \in A \rtimes \mathcal{G}$;

- $\alpha_{A \rtimes \mathcal{G}}(n):=\widehat{\theta}(\widehat{\alpha}(n))=\left.\left(1_{A} \otimes \widehat{\alpha}(n)\right)\right|_{\mathcal{E}_{A, L^{\prime}}}$ for all $n \in N$.

Then, $\delta_{A \rtimes \mathcal{G}}$ is a faithful ${ }^{*}$-homomorphism and $\alpha_{A \rtimes \mathcal{G}}$ is a non-degenerate ${ }^{*}$-homomorphism. Moreover, we have the following statements:

1. $\delta_{A \rtimes \mathcal{G}}(\pi(a) \widehat{\theta}(x))=\left(\pi(a) \otimes 1_{\widehat{S}}\right)\left(\widehat{\theta} \otimes \mathrm{id}_{\widehat{S}}\right) \widehat{\delta}(x)$, for all $a \in A$ and $x \in \widehat{S}$; in particular, $\delta_{A \rtimes \mathcal{G}}$ takes its values in $\mathcal{M}((A \rtimes \mathcal{G}) \otimes \widehat{S})$;

2. $\alpha_{A \rtimes \mathcal{G}}(n) \pi(a) \widehat{\theta}(x)=\pi(a) \widehat{\theta}(\widehat{\alpha}(n) x)$ and $\pi(a) \widehat{\theta}(x) \alpha_{A \rtimes \mathcal{G}}(n)=\pi(a) \widehat{\theta}(x \widehat{\alpha}(n))$ for all $n \in$ $N, a \in A$ and $x \in \widehat{S}$.

5.1.16 Proposition-Definition. - With the notations of 5.1.15, the pair $\left(\alpha_{A \rtimes \mathcal{G}}, \delta_{A \rtimes \mathcal{G}}\right)$ is a strongly continuous action of $\widehat{\mathcal{G}}$ on $A \rtimes \mathcal{G}$ called the dual action of $\left(\beta_{A}, \delta_{A}\right)$.

In a similar way, we define the crossed product of a $C^{*}$-algebra $B$ by a strongly continuous action $\left(\alpha_{B}, \delta_{B}\right)$ of the dual measured quantum groupoid $\widehat{\mathcal{G}}$.

5.1.17 Notations. - The *-representation

$$
\widehat{\pi}_{\lambda}:=\left(\operatorname{id}_{B} \otimes \lambda\right) \circ \delta_{B}: B \rightarrow \mathcal{L}(B \otimes \mathscr{H})
$$

of $B$ on the Hilbert $B$-module $B \otimes \mathscr{H}$ extends uniquely to a strictly $/{ }^{*}$-strongly continuous faithful *-representation $\widehat{\pi}_{\lambda}: \mathcal{M}(B) \rightarrow \mathcal{L}(B \otimes \mathscr{H})$ such that $\widehat{\pi}_{\lambda}\left(1_{B}\right)=q_{\alpha_{B} \widehat{\beta}}$. Moreover, we have $\widehat{\pi}_{\lambda}(m)=\widehat{\pi}_{\lambda}(m) q_{\alpha_{B} \widehat{\beta}}=q_{\alpha_{B} \widehat{\beta}} \widehat{\pi}_{\lambda}(m)$, for all $m \in \mathcal{M}(B)$. Consider the Hilbert $B$-module

$$
\mathcal{E}_{B, \lambda}:=q_{\alpha_{B} \widehat{\beta}}(B \otimes \mathscr{H}) .
$$

By restricting $\widehat{\pi}_{\lambda}$, we obtain a strictly $/{ }^{*}$-strongly continuous faithful unital ${ }^{*}$-representation

$$
\widehat{\pi}: \mathcal{M}(B) \rightarrow \mathcal{L}\left(\mathcal{E}_{B, \lambda}\right) ;\left.m \mapsto \widehat{\pi}_{\lambda}(m)\right|_{\mathcal{E}_{B, \lambda}} .
$$

We have $\left[1_{B} \otimes T, q_{\alpha_{B} \widehat{\beta}}\right]=0$ for all $T \in \mathcal{M}(S)$. We then obtain a strictly/*-strongly continuous unital ${ }^{*}$-representation

$$
\theta: \mathcal{M}(S) \rightarrow \mathcal{L}\left(\mathcal{E}_{B, \lambda}\right) ;\left.T \mapsto\left(1_{B} \otimes T\right)\right|_{\mathcal{E}_{B, \lambda}} .
$$

Note that if $\alpha_{B}$ is faithful, then so is $\theta$.

5.1.18 Proposition-Definition. - The norm closed subspace of $\mathcal{L}\left(\mathcal{E}_{B, \lambda}\right)$ spanned by the products of the form $\widehat{\pi}(b) \theta(x)$ for $b \in B$ and $x \in S$ is a $C^{*}$-subalgebra called the (reduced) crossed product of $B$ by the strongly continuous action $\left(\alpha_{B}, \delta_{B}\right)$ of $\widehat{\mathcal{G}}$ and denoted by $B \rtimes \widehat{\mathcal{G}}$.

In particular, the morphism $\widehat{\pi}$ (resp. $\theta$ ) defines a faithful unital *-homomorphism (resp. unital ${ }^{*}$-homomorphism) $\widehat{\pi}: \mathcal{M}(B) \rightarrow \mathcal{M}(B \rtimes \widehat{\mathcal{G}})($ resp. $\theta: S \rightarrow \mathcal{M}(B \rtimes \widehat{\mathcal{G}}))$. 
Since $\left[V, \beta\left(n^{\mathrm{o}}\right) \otimes 1\right]=0$, we have $\left[V_{23}, q_{\alpha_{B} \beta, 12}\right]=0$. The operator $V_{23} \in \mathcal{L}(B \otimes \mathscr{H} \otimes \mathscr{H})$ restricts to a partial isometry

$$
Y:=V_{23}\left\lceil\mathcal{E}_{B, \lambda} \otimes \mathscr{H} \in \mathcal{L}\left(\mathcal{E}_{B, \lambda} \otimes \mathscr{H}\right),\right.
$$

whose initial and final projections are $Y^{*} Y=q_{\widehat{\alpha} \beta, 23}\left\lceil\mathcal{E}_{B, \lambda} \otimes \mathscr{H}\right.$ and $Y Y^{*}=q_{\beta \alpha, 23}\left\lceil\mathcal{E}_{B, \lambda} \otimes \mathscr{H}\right.$.

5.1.19 Proposition-Definition. - Let

$$
\delta_{B \rtimes \widehat{\mathcal{G}}}: B \rtimes \widehat{\mathcal{G}} \rightarrow \mathcal{L}\left(\mathcal{E}_{B, \lambda} \otimes \mathscr{H}\right) \quad \text { and } \quad \beta_{B \rtimes \widehat{\mathcal{G}}}: N^{\mathrm{o}} \rightarrow \mathcal{L}\left(\mathcal{E}_{B, \lambda}\right)
$$

be the linear maps defined by:

- $\delta_{B \rtimes \widehat{\mathcal{G}}}(c):=Y\left(c \otimes 1_{\mathscr{H}}\right) Y^{*}$, for all $c \in B \rtimes \widehat{\mathcal{G}} ;$

- $\beta_{B \rtimes \widehat{\mathcal{G}}}\left(n^{\mathrm{o}}\right):=\theta\left(\beta\left(n^{\mathrm{o}}\right)\right)=\left(1_{B} \otimes \beta\left(n^{\mathrm{o}}\right)\right) \uparrow_{\mathcal{E}_{B, \lambda} \otimes \mathscr{H}}$, for all $n \in N$.

Then, $\delta_{B \rtimes \widehat{\mathcal{G}}}$ is a faithful *-homomorphism and $\beta_{B \rtimes \widehat{\mathcal{G}}}$ is a non-degenerate ${ }^{*}$-homomorphism. Moreover, we have the following statements:

1. $\delta_{B \rtimes \widehat{\mathcal{G}}}(\widehat{\pi}(b) \theta(x))=\left(\widehat{\pi}(b) \otimes 1_{S}\right)\left(\theta \otimes \mathrm{id}_{S}\right) \delta(x)$, for all $b \in B$ and $x \in S$; in particular, $\delta_{B \rtimes \widehat{\mathcal{G}}}$ takes its values in $\mathcal{M}((B \rtimes \widehat{\mathcal{G}}) \otimes S)$;

2. $\beta_{B \rtimes \widehat{\mathcal{G}}}\left(n^{\mathrm{o}}\right) \widehat{\pi}(b) \theta(x)=\widehat{\pi}(b) \theta\left(\beta\left(n^{\mathrm{o}}\right) x\right)$ and $\widehat{\pi}(b) \theta(x) \beta_{B \rtimes \widehat{\mathcal{G}}}\left(n^{\mathrm{o}}\right)=\widehat{\pi}(b) \theta\left(x \beta\left(n^{\mathrm{o}}\right)\right)$ for all $n \in N, b \in B$ and $x \in S$.

5.1.20 Proposition-Definition. - With the notations of 5.1.19. the pair $\left(\beta_{B \rtimes \widehat{\mathcal{G}}}, \delta_{B \rtimes \widehat{\mathcal{G}}}\right)$ is a strongly continuous action of $\mathcal{G}$ on $B \rtimes \widehat{\mathcal{G}}$ called the dual action of $\left(\alpha_{B}, \delta_{B}\right)$.

\subsubsection{Takesaki-Takai duality}

Let $\left(\beta_{A}, \delta_{A}\right)$ be a strongly continuous action of the groupoid $\mathcal{G}$ on a $C^{*}$-algebra $A$.

5.1.21 Notations. - The *-representation

$$
\pi_{R}:=\left(\mathrm{id}_{A} \otimes R\right) \circ \delta_{A}: A \rightarrow \mathcal{L}(A \otimes \mathscr{H})
$$

of $A$ on the Hilbert $A$-module $A \otimes \mathscr{H}$ extends uniquely to a strictly /*-strongly continuous faithful ${ }^{*}$-representation $\pi_{R}: \mathcal{M}(A) \rightarrow \mathcal{L}(A \otimes \mathscr{H})$ satisfying $\pi_{R}(m)=\left(\mathrm{id}_{A} \otimes R\right) \delta_{A}(m)$ for all $m \in \mathcal{M}(A)$ and $\pi_{R}\left(1_{A}\right)=q_{\beta_{A} \widehat{\alpha}}$. Consider the Hilbert $A$-module

$$
\mathcal{E}_{A, R}:=q_{\beta_{A} \widehat{\alpha}}(A \otimes \mathscr{H}) .
$$

We recall that the Banach space

$$
D:=\left[\pi_{R}(a)\left(1_{A} \otimes \lambda(x) L(y)\right) ; a \in A, x \in \widehat{S}, y \in S\right]
$$

is a $C^{*}$-subalgebra of $\mathcal{L}(A \otimes \mathscr{H})$ such that $d q_{\beta_{A} \widehat{\alpha}}=d=d q_{\beta_{A} \widehat{\alpha}}$ for all $d \in D$. Moreover, we have $D(A \otimes \mathscr{H})=\mathcal{E}_{A, R}$. We also recall that there exists a unique strictly $/^{*}$-strongly continuous faithful *-representation $j_{D}: \mathcal{M}(D) \rightarrow \mathcal{L}(A \otimes \mathscr{H})$ extending the inclusion map $D \subset \mathcal{L}(A \otimes \mathscr{H})$ such that $j_{D}\left(1_{D}\right)=q_{\beta_{A} \widehat{\alpha}}$.

5.1.22 Proposition. - There exists a unique ${ }^{*}$-isomorphism $\phi:(A \rtimes \mathcal{G}) \rtimes \widehat{\mathcal{G}} \rightarrow D$ such that $\phi(\widehat{\pi}(\pi(a) \widehat{\theta}(x)) \theta(y))=\pi_{R}(a)\left(1_{A} \otimes \lambda(x) L(y)\right)$ for all $a \in A, x \in \widehat{S}$ and $y \in S$.

5.1.23 Notations. - We denote $\mathcal{K}:=\mathcal{K}(\mathscr{H})$ for short. Let $\delta_{0}: A \otimes \mathcal{K} \rightarrow \mathcal{M}(A \otimes \mathcal{K} \otimes S)$ be the *-homomorphism defined by $\delta_{0}(a \otimes k)=\delta_{A}(a)_{13}\left(1_{A} \otimes k \otimes 1_{S}\right)$ for all $a \in A$ and $k \in \mathcal{K}$. The morphism $\delta_{0}$ extends uniquely to a strictly continuous *-homomorphism $\delta_{0}: \mathcal{M}(A \otimes \mathcal{K}) \rightarrow \mathcal{M}(A \otimes \mathcal{K} \otimes S)$ such that $\delta_{0}\left(1_{A \otimes \mathcal{K}}\right)=q_{\beta_{A} \alpha, 13}$. Let $\mathcal{V} \in \mathcal{L}(\mathscr{H} \otimes S)$ be the unique partial isometry such that $\left(\mathrm{id}_{\mathcal{K}} \otimes L\right)(\mathcal{V})=V$.

5.1.24 THEOREM. - There exists a unique strongly continuous action $\left(\beta_{D}, \delta_{D}\right)$ of $\mathcal{G}$ on the $C^{*}$-algebra $D=\left[\pi_{R}(a)\left(1_{A} \otimes \lambda(x) L(y)\right) ; a \in A, x \in \widehat{S}, y \in S\right]$ defined by the relations:

$$
\left(j_{D} \otimes \mathrm{id}_{S}\right) \delta_{D}(d)=\mathcal{V}_{23} \delta_{0}(d) \mathcal{V}_{23}^{*}, \quad d \in D ; \quad j_{D}\left(\beta_{D}\left(n^{\mathrm{o}}\right)\right)=q_{\beta_{A} \widehat{\alpha}}\left(1_{A} \otimes \beta\left(n^{\mathrm{o}}\right)\right), \quad n \in N .
$$

Moreover, the canonical ${ }^{*}$-isomorphism $\phi:(A \rtimes \mathcal{G}) \rtimes \widehat{\mathcal{G}} \rightarrow D(c f .5 .1 .22)$ is $\mathcal{G}$-equivariant. If the groupoid $\mathcal{G}$ is regular, then we have $D=q_{\beta_{A} \widehat{\alpha}}(A \otimes \mathcal{K}) q_{\beta_{A} \widehat{\alpha}}$.

The $\mathcal{G}$-C ${ }^{*}$-algebra $D$ defined above will be referred to as the bidual $\mathcal{G}-C^{*}$-algebra of $A$. 


\subsection{Case of a colinking measured quantum groupoid}

In this section, we fix a colinking measured quantum groupoid $\mathcal{G}:=\mathcal{G}_{\mathrm{G}_{1}, \mathrm{G}_{2}}$ associated with two monoidally equivalent locally compact quantum groups $\mathbb{G}_{1}$ and $G_{2}$. We follow all the notations recalled in $\$ 3.3$ concerning the objects associated with $\mathcal{G}$.

In the following, we recall the notations and the main results of \$3.2.3 [2] concerning the equivalent description of the $\mathcal{G}$ - $\mathrm{C}^{*}$-algebras in terms of $\mathrm{G}_{1}$ - $\mathrm{C}^{*}$-algebras and $\mathrm{G}_{2}$ - $\mathrm{C}^{*}$-algebras. Let us fix a $\mathcal{G}$-C*-algebra $\left(A, \beta_{A}, \delta_{A}\right)$.

5.2.1 Notations. - - The morphism $\beta_{A}: \mathbb{C}^{2} \rightarrow \mathcal{M}(A)$ is central. Let $q_{j}:=\beta_{A}\left(\varepsilon_{j}\right)$ for $j=1,2$. Then, $q_{j}$ is a central self-adjoint projection of $\mathcal{M}(A)$ and $q_{1}+q_{2}=1_{A}$. Let $A_{j}:=q_{j} A$ for $j=1,2$. For $j=1,2, A_{j}$ is a $C^{*}$-subalgebra (actually a closed two-sided ideal) of $A$ and we have $A=A_{1} \oplus A_{2}$.

- For $j, k=1,2$, let $\pi_{j}^{k}: \mathcal{M}\left(A_{k} \otimes S_{k j}\right) \rightarrow \mathcal{M}(A \otimes S)$ be the unique strictly continuous extension of the inclusion $A_{k} \otimes S_{k j} \subset A \otimes S$ such that $\pi_{j}^{k}\left(1_{A_{k} \otimes S_{k j}}\right)=q_{k} \otimes p_{k j}$.

In case of ambiguity, we will denote $\pi_{A, j}^{k}$ and $q_{A, j}$ instead of $\pi_{j}^{k}$ and $q_{j}$.

5.2.2 Proposition. - For all $j, k=1,2$, there exists a unique faithful non-degenerate *-homomorphism

$$
\delta_{A_{j}}^{k}: A_{j} \rightarrow \mathcal{M}\left(A_{k} \otimes S_{k j}\right)
$$

such that for all $x \in A_{j}$, we have

$$
\pi_{j}^{k} \circ \delta_{A_{j}}^{k}(x)=\left(q_{k} \otimes p_{k j}\right) \delta_{A}(x)=\left(q_{k} \otimes 1_{S}\right) \delta_{A}(x)=\left(1_{A} \otimes \alpha\left(\varepsilon_{k}\right)\right) \delta_{A}(x)=\left(1_{A} \otimes p_{k j}\right) \delta_{A}(x) .
$$

Moreover, we have:

1. $\delta_{A}(a)=\sum_{k, j=1,2} \pi_{j}^{k} \circ \delta_{A_{j}}^{k}\left(q_{j} a\right)$, for all $a \in A$;

2. $\left(\delta_{A_{k}}^{l} \otimes \mathrm{id}_{S_{k j}}\right) \delta_{A_{j}}^{k}=\left(\mathrm{id}_{A_{l}} \otimes \delta_{l j}^{k}\right) \delta_{A_{j}}^{l}$ for all $j, k, l=1,2$;

3. $\left[\delta_{A_{j}}^{k}\left(A_{j}\right)\left(1_{A_{k}} \otimes S_{k j}\right)\right]=A_{k} \otimes S_{k j}$, for all $j, k=1,2$; in particular, we have

$$
A_{k}=\left[\left(\operatorname{id}_{A_{k}} \otimes \omega\right) \delta_{A_{j}}^{k}(a) ; a \in A_{j}, \omega \in \mathcal{B}\left(\mathscr{H}_{k j}\right)_{*}\right] ;
$$

4. $\delta_{A_{j}}^{j}: A_{j} \rightarrow \mathcal{M}\left(A_{j} \otimes S_{j j}\right)$ is a strongly continuous action of $\mathrm{G}_{j}$ on $A_{j}$.

From this concrete description of $\mathcal{G}$ - $\mathrm{C}^{*}$-algebras we can also give a convenient description of the $\mathcal{G}$-equivariant ${ }^{*}$-homomorphisms. With the above notations, we have the result below.

5.2.3 Proposition. - Let $A$ and $B$ be two $\mathcal{G}$-C $C^{*}$-algebras. For $k=1,2$, let $\iota_{k}: \mathcal{M}\left(B_{k}\right) \rightarrow \mathcal{M}(B)$ be the unique strictly continuous extension of the inclusion map $B_{k} \subset B$ such that $\iota_{k}\left(1_{B_{k}}\right)=q_{B, k}$.

1. Let $f: A \rightarrow \mathcal{M}(B)$ be a non-degenerate $\mathcal{G}$-equivariant *-homomorphism. Then, for all $j=1,2$, there exists a unique non-degenerate ${ }^{*}$-homomorphism $f_{j}: A_{j} \rightarrow \mathcal{M}\left(B_{j}\right)$ such that for $k=1,2$ we have

$$
\left(f_{k} \otimes \mathrm{id}_{S_{k j}}\right) \circ \delta_{A_{j}}^{k}=\delta_{B_{j}}^{k} \circ f_{j} .
$$

Moreover, we have $f(a)=\iota_{1} \circ f_{1}\left(a q_{A, 1}\right)+\iota_{2} \circ f_{2}\left(a q_{A, 2}\right)$ for all $a \in A$.

2. Conversely, for $j=1,2$ let $f_{j}: A_{j} \rightarrow \mathcal{M}\left(B_{j}\right)$ be a non-degenerate *-homomorphism such that (5.1) holds for all $j, k=1,2$. Then, the map $f: A \rightarrow \mathcal{M}(B)$, defined for all $a \in A$ by

$$
f(a):=\iota_{1} \circ f_{1}\left(a q_{A, 1}\right)+\iota_{2} \circ f_{2}\left(a q_{A, 2}\right),
$$

is a non-degenerate $\mathcal{G}$-equivariant *-homomorphism.

The above results show that for $j=1,2$ we have a functor 


$$
\operatorname{Alg}_{\mathcal{G}} \rightarrow \operatorname{Alg}_{\mathbb{G}_{j}} ;\left(A, \beta_{A}, \delta_{A}\right) \mapsto\left(A_{j}, \delta_{A_{j}}^{j}\right) .
$$

In $\S_{4}$ [2], it has been proved that if $\mathcal{G}$ is regular (cf. $4 \cdot 5$, then $\left(A, \delta_{A}, \beta_{A}\right) \rightarrow\left(A_{1}, \delta_{A_{1}}^{1}\right)$ is an equivalence of categories. Moreover, the authors build explicitly the inverse functor $\left(A_{1}, \delta_{A_{1}}\right) \rightarrow\left(A, \beta_{A}, \delta_{A}\right)$. More precisely, to any $\mathrm{G}_{1}-\mathrm{C}^{*}$-algebra $\left(A_{1}, \delta_{A_{1}}\right)$ they associate a $\mathrm{G}_{2}-\mathrm{C}^{*}$-algebra $\left(A_{2}, \delta_{A_{2}}\right)$ in a canonical way. Then, the $\mathrm{C}^{*}$-algebra $A:=A_{1} \oplus A_{2}$ can be equipped with a strongly continuous action $\left(\beta_{A}, \delta_{A}\right)$ of the groupoid $\mathcal{G}$. This allowed them to build the inverse functor $\left(A_{1}, \delta_{A_{1}}\right) \rightarrow\left(A, \beta_{A}, \delta_{A}\right)$. The equivalence of categories $\left(A_{1}, \delta_{A_{1}}\right) \rightarrow\left(A_{2}, \delta_{A_{2}}\right)$ generalizes the correspondence of actions for monoidally equivalent compact quantum groups of De Rijdt and Vander Vennet [15]. We bring to the reader's attention that an induction procedure has been developed by De Commer in the von Neumann algebraic setting (cf. §8 [12]).

In the following, we recall the notations and the main results of $\S_{4}[2]$. We assume that the quantum groups $\mathbb{G}_{1}$ and $G_{2}$ are regular.

5.2.4 Notations. - Let $\delta_{A_{1}}: A_{1} \rightarrow \mathcal{M}\left(A_{1} \otimes S_{11}\right)$ be a continuous action of $\mathrm{G}_{1}$ on a $C^{*}$-algebra $A_{1}$. Let us denote:

$$
\delta_{A_{1}}^{1}:=\delta_{A_{1}}, \quad \delta_{A_{1}}^{(2)}:=\left(\mathrm{id}_{A_{1}} \otimes \delta_{11}^{2}\right) \delta_{A_{1}}: A_{1} \rightarrow \mathcal{M}\left(A_{1} \otimes S_{12} \otimes S_{21}\right) .
$$

Then, $\delta_{A_{1}}^{(2)}$ is a faithful non-degenerate ${ }^{*}$-homomorphism. In the following, we will identify $S_{21}$ with a $\mathrm{C}^{*}$-subalgebra of $\mathcal{B}\left(\mathscr{H}_{21}\right)$. Let

$$
\operatorname{Ind}_{\mathrm{G}_{1}}^{\mathrm{G}_{2}}\left(A_{1}\right):=\left[\left(\operatorname{id}_{A_{1} \otimes S_{12}} \otimes \omega\right) \delta_{A_{1}}^{(2)}(a) ; a \in A_{1}, \omega \in \mathcal{B}\left(\mathscr{H}_{21}\right)_{*}\right] \subset \mathcal{M}\left(A_{1} \otimes S_{12}\right) .
$$

5.2.5 Proposition. - The Banach space $A_{2}:=\operatorname{Ind}_{\mathrm{G}_{1}}^{\mathrm{G}_{2}}\left(A_{1}\right) \subset \mathcal{M}\left(A_{1} \otimes S_{12}\right)$ is a $C^{*}$-algebra. Moreover, we have:

1. $\left[A_{2}\left(1_{A_{1}} \otimes S_{12}\right)\right]=A_{1} \otimes S_{12}=\left[\left(1_{A_{1}} \otimes S_{12}\right) A_{2}\right] ;$ in particular, $A_{2} \subset \mathcal{M}\left(A_{1} \otimes S_{12}\right)$ defines a faithful non-degenerate *-homomorphism and $\mathcal{M}\left(A_{2}\right) \subset \mathcal{M}\left(A_{1} \otimes S_{12}\right)$;

2. let $\delta_{A_{2}}:=\left(\mathrm{id}_{A_{1}} \otimes \delta_{12}^{2}\right) \Gamma_{A_{2}}$, we have $\delta_{A_{2}}\left(A_{2}\right) \subset \mathcal{M}\left(A_{2} \otimes S_{22}\right)$ and $\delta_{A_{2}}$ is a continuous action of $\mathrm{G}_{2}$ on $A_{2}$;

3. the correspondence $\operatorname{Ind}_{\mathrm{G}_{1}}^{\mathrm{G}_{2}}: \operatorname{Alg}_{\mathrm{G}_{1}} \rightarrow \mathrm{Alg}_{\mathrm{G}_{2}}$ is functorial.

By exchanging the roles of the quantum groups $\mathrm{G}_{1}$ and $\mathrm{G}_{2}$, we obtain mutatis mutandis a functor $\operatorname{Ind}_{\mathrm{G}_{2}}^{\mathrm{G}_{1}}: \mathrm{Alg}_{\mathrm{G}_{2}} \rightarrow \operatorname{Alg}_{\mathrm{G}_{1}}$.

5.2.6 Proposition. - Let $j, k=1,2$ with $j \neq k$. Let $\left(A_{j}, \delta_{A_{j}}\right)$ be a $\mathbb{G}_{j}$-C -algebra. Let

$$
A_{k}:=\operatorname{Ind}_{\mathbb{G}_{j}}^{\mathrm{G}_{k}}\left(A_{j}\right) \subset \mathcal{M}\left(A_{j} \otimes S_{j k}\right) \quad \text { and } \quad C:=\operatorname{Ind}_{\mathbb{G}_{k}}^{\mathrm{G}_{j}}\left(A_{k}\right) \subset \mathcal{M}\left(A_{k} \otimes S_{k j}\right)
$$

endowed with the continuous actions $\delta_{A_{k}}:=\left(\mathrm{id}_{A_{j}} \otimes \delta_{j k}^{k}\right) \uparrow_{A_{k}}$ and $\delta_{C}:=\left(\mathrm{id}_{A_{k}} \otimes \delta_{k j}^{j}\right) \Gamma_{C}$ respectively. Then, we have:

1. $C \subset \mathcal{M}\left(A_{k} \otimes S_{k j}\right) \subset \mathcal{M}\left(A_{j} \otimes S_{j k} \otimes S_{k j}\right)$ and $C=\delta_{A_{j}}^{(k)}\left(A_{j}\right) ;$

2. $\pi_{j}: A_{j} \rightarrow C ; a \mapsto \delta_{A_{j}}^{(k)}(a):=\left(\operatorname{id}_{A_{j}} \otimes \delta_{j j}^{k}\right) \delta_{A_{j}}(a)$ is a $\mathrm{G}_{j}$-equivariant ${ }^{*}$-isomorphism;

3. $\delta_{A_{j}}^{k}: A_{j} \rightarrow \mathcal{M}\left(A_{k} \otimes S_{k j}\right) ; a \mapsto \delta_{A_{j}}^{(k)}(a):=\left(\operatorname{id}_{A_{j}} \otimes \delta_{j j}^{k}\right) \delta_{A_{j}}(a)$ is a faithful non-degenerate *homomorphism.

The above result shows that the functors $\operatorname{Ind}_{\mathrm{G}_{1}}^{\mathrm{G}_{2}}$ and $\operatorname{Ind}_{\mathrm{G}_{2}}^{\mathrm{G}_{1}}$ are inverse of each other. 
5.2.7 Notations. - Let $\left(B_{1}, \delta_{B_{1}}\right)$ be a $\mathbb{G}_{1}-C^{*}$-algebra. Let $\left(B_{2}, \delta_{B_{2}}\right)$ be the induced $\mathbb{G}_{2}-C^{*}$ algebra, that is to say $B_{2}=\operatorname{Ind}_{\mathrm{G}_{1}}^{\mathrm{G}_{2}}\left(B_{1}\right)$ and $\delta_{B_{2}}=\left(\mathrm{id}_{B_{1}} \otimes \delta_{12}^{2}\right) \Gamma_{B_{2}}$. In virtue of 5.2.6, we have four *-homomorphisms:

$$
\delta_{B_{j}}^{k}: B_{j} \rightarrow \mathcal{M}\left(B_{k} \otimes S_{k j}\right), \quad j, k=1,2
$$

Let us give a precise description of them. We denote $\delta_{B_{1}}^{1}:=\delta_{B_{1}}$ and $\delta_{B_{2}}^{2}:=\delta_{B_{2}}$. The *-homomorphism $\delta_{B_{1}}^{2}: B_{1} \rightarrow \mathcal{M}\left(B_{2} \otimes S_{21}\right)$ is given by

$b \in B_{1} \mapsto \delta_{B_{1}}^{2}(b):=\delta_{B_{1}}^{(2)}(b) \in \mathcal{M}\left(B_{2} \otimes S_{21}\right) \quad\left(\right.$ with $\delta_{B_{1}}^{(2)}(b):=\left(\operatorname{id}_{B_{1}} \otimes \delta_{11}^{2}\right) \delta_{B_{1}}^{1}(b)$, for $\left.b \in B_{1}\right)$ whereas the ${ }^{*}$-homomorphism $\delta_{B_{2}}^{1}: B_{2} \rightarrow \mathcal{M}\left(B_{1} \otimes S_{12}\right)$ is defined by the relation

$$
\left(\pi_{1} \otimes \operatorname{id}_{S_{12}}\right) \delta_{B_{2}}^{1}(b)=\delta_{B_{2}}^{(1)}(b) \quad \text { for } b \in B_{2},
$$

where $\delta_{B_{2}}^{(1)}:=\left(\operatorname{id}_{B_{2}} \otimes \delta_{22}^{1}\right) \delta_{B_{2}}^{2}$ and $\pi_{1}: B_{1} \rightarrow \operatorname{Ind}_{\mathbb{G}_{2}}^{\mathbb{G}_{1}}\left(B_{2}\right) ; b \mapsto \delta_{B_{1}}^{(2)}(b)$ (cf. 5.2.6 2).

5.2.8 Proposition. - Let $\left(A, \beta_{A}, \delta_{A}\right)$ be a $\mathcal{G}$-C*-algebra. Let $j, k=1,2$ with $j \neq k$. With the notations of 5.2.2. let

$$
\left(\widetilde{A}_{j}, \delta_{\widetilde{A}_{j}}\right):=\operatorname{Ind}_{\mathrm{G}_{k}}^{\mathrm{G}_{j}}\left(A_{k}, \delta_{A_{k}}^{k}\right) .
$$

If $x \in A_{j}$, then we have $\delta_{A_{j}}^{k}(x) \in \widetilde{A}_{j} \subset \mathcal{M}\left(A_{k} \otimes S_{k j}\right)$ and the map $\widetilde{\pi}_{j}: A_{j} \rightarrow \widetilde{A}_{j} ; x \mapsto \delta_{A_{j}}^{k}(x)$ is a $\mathrm{G}_{j}$-equivariant ${ }^{*}$-isomorphism.

5.2.9 Proposition. - Let $\left(B_{1}, \delta_{B_{1}}\right)$ be a $\mathrm{G}_{1}-C^{*}$-algebra. Let $B_{2}=\operatorname{Ind}_{\mathrm{G}_{1}}^{\mathrm{G}_{2}}\left(B_{1}\right)$ be the induced $\mathbb{G}_{2}-C^{*}$-algebra. Let $B:=B_{1} \oplus B_{2}$. For $j, k=1,2$ with $j \neq k$, let $\pi_{j}^{k}: \mathcal{M}\left(B_{k} \otimes S_{k j}\right) \rightarrow \mathcal{M}(B \otimes S)$ be the strictly continuous ${ }^{*}$-homomorphism extending the canonical injection $B_{k} \otimes S_{k j} \rightarrow B \otimes S$ and $\delta_{B_{j}}^{k}: B_{j} \rightarrow \mathcal{M}\left(B_{k} \otimes S_{k j}\right)$ the *-homomorphisms defined in 5.2.7 Let $\beta_{B}: \mathbb{C}^{2} \rightarrow \mathcal{M}(B)$ and $\delta_{B}: B \rightarrow \mathcal{M}(B \otimes S)$ be the *-homomorphisms defined by:

$$
\beta_{B}(\lambda, \mu):=\left(\begin{array}{ll}
\lambda & 0 \\
0 & \mu
\end{array}\right), \quad(\lambda, \mu) \in \mathbb{C}^{2} ; \quad \delta_{B}(b):=\sum_{k, j=1,2} \pi_{j}^{k} \circ \delta_{B_{j}}^{k}\left(b_{j}\right), \quad b=\left(b_{1}, b_{2}\right) \in B .
$$

Therefore, we have:

1. $\left(\beta_{B}, \delta_{B}\right)$ is a strongly continuous action of $\mathcal{G}$ on $B$;

2. the correspondence $\operatorname{Alg}_{G_{1}} \rightarrow \operatorname{Alg}_{\mathcal{G}} ;\left(B_{1}, \delta_{B_{1}}\right) \mapsto\left(B, \beta_{B}, \delta_{B}\right)$ is functorial;

3. the functors $\mathrm{Alg}_{\mathrm{G}_{1}} \rightarrow \mathrm{Alg}_{\mathcal{G}}$ and $\mathrm{Alg}_{\mathcal{G}} \rightarrow \mathrm{Alg}_{\mathrm{G}_{1}}$ are inverse of each other.

\subsection{Actions of (semi-)regular measured quantum groupoids}

In this section, we fix a measured quantum groupoid $\mathcal{G}=\left(N, M, \alpha, \beta, \Delta, T, T^{\prime}, \epsilon\right)$ on a finite-dimensional basis $N:=\bigoplus_{1 \leqslant l \leqslant k} \mathrm{M}_{n_{l}}(\mathbb{C})$ endowed with the non-normalized Markov trace $\epsilon=\bigoplus_{1 \leqslant l \leqslant k} n_{l} \cdot \operatorname{Tr}_{l}$ and we use all the notations introduced in 83.1 .

We begin this section by a characterization of the regularity (resp. semi-regularity) of $\mathcal{G}$ in terms of the action of $\mathcal{G}$ on itself (cf. 5.1.4), which generalizes 2.6 [5] to the setting of measured quantum groupoids on a finite basis.

5.3.1 Proposition. - Let $S \rtimes \mathcal{G}$ be the crossed product of $S$ by the strongly continuous action $(\beta, \delta)$ of $\mathcal{G}$. Then, we have a canonical ${ }^{*}$-isomorphism $S \rtimes \mathcal{G} \simeq[S \widehat{S}]$. In particular, $\mathcal{G}$ is regular (resp. semi-regular) if, and only if, we have $\mathcal{K}_{\widehat{\beta}}=S \rtimes \mathcal{G}$ (resp. $\left.\mathcal{K}_{\widehat{\beta}} \subset S \rtimes \mathcal{G}\right)$.

Proof. Let us identify $\mathcal{L}\left(\mathcal{E}_{S, L}\right)=\left\{T \in \mathcal{L}(S \otimes \mathscr{H}) ; T q_{\beta \alpha}=T=q_{\beta \alpha} T\right\}$. Let us denote by $j_{S \rtimes \mathcal{G}}: S \rtimes \mathcal{G} \rightarrow \mathcal{B}(\mathscr{H} \otimes \mathscr{H})$, the faithful ${ }^{*}$-representation defined by $j_{S \rtimes \mathcal{G}}(u)=\left(L \otimes \operatorname{id}_{\mathcal{K}}\right)(u)$ for all $u \in S \rtimes \mathcal{G} \subset \mathcal{L}\left(\mathcal{E}_{S, L}\right)$. Let $\pi: S \rightarrow \mathcal{M}(S \rtimes \mathcal{G})$ and $\widehat{\theta}: \widehat{S} \rightarrow \mathcal{M}(S \rtimes \mathcal{G})$ be the canonical 
morphisms (cf. 5.1.13). We claim that there exists a unique ${ }^{*}$-isomorphism $\phi: S \rtimes \mathcal{G} \rightarrow[S \widehat{S}]$ such that

$$
\phi(\pi(s) \widehat{\theta}(x))=L(s) \rho(x), \quad \text { for all } s \in S \text { and } x \in \widehat{S} .
$$

Let $s \in S$ and $x \in \widehat{S}$. Since $W \in M \otimes \widehat{M}$ and $\rho(\widehat{S}) \subset \widehat{M}^{\prime}$, we have

$$
\begin{aligned}
j_{S \rtimes \mathcal{G}}(\pi(s) \widehat{\theta}(x)) & =(L \otimes L) \delta(s)(1 \otimes \rho(x)) \\
& =W^{*}(1 \otimes L(s)) W(1 \otimes \rho(x)) \\
& =W^{*}(1 \otimes L(s) \rho(x)) W .
\end{aligned}
$$

Let $C:=\operatorname{im}\left(j_{S \rtimes \mathcal{G}}\right)=\left\{W^{*}(1 \otimes z) W ; z \in[S \widehat{S}]\right\}$. The representation $j_{S \rtimes \mathcal{G}}$ induces a *isomorphism $\psi: S \rtimes \mathcal{G} \rightarrow C$. Since $W W^{*}=q_{\alpha \widehat{\beta}}$ and $\left[1 \otimes z, q_{\alpha \widehat{\beta}}\right]=0$ for all $z \in[S \widehat{S}]$, the map

$$
\chi:[S \widehat{S}] \rightarrow C ; z \rightarrow W^{*}(1 \otimes z) W
$$

is a ${ }^{*}$-homomorphism satisfying $W \chi(z) W^{*}=q_{\alpha \widehat{\beta}}(1 \otimes z)$ for all $z \in[S \widehat{S}]$. Let $\omega \in \mathcal{B}(\mathscr{H})_{*}$ such that $\omega \circ \alpha=\epsilon$. We have

$$
(\omega \otimes \mathrm{id})\left(W \chi(z) W^{*}\right)=z, \quad \text { for all } z \in[S \widehat{S}] .
$$

Hence, $\chi$ is a ${ }^{*}$-isomorphism. Hence, $\phi:=\chi^{-1} \circ \psi: S \rtimes \mathcal{G} \rightarrow[S \widehat{S}] ; \pi(s) \widehat{\theta}(x) \mapsto L(s) \widehat{\theta}(x)$ is a ${ }^{*}$-isomorphism. The second statement of the proposition follows from 4.4

Proposition 5.3.4 and Theorem 5.3.6 are the generalizations of 5.7 and 5.8 of [5] to measured quantum groupoids on a finite basis.

5.3.2 Notations. - Let $\left(\beta_{A}, \delta_{A}\right)$ be an action of $\mathcal{G}$ on a $C^{*}$-algebra $A$. With the notations of 8.2.21 and 8.2.22, let $e_{I}:=\alpha\left(\varepsilon_{I}\right)$ and $q_{I}:=\beta_{A}\left(\varepsilon_{I}\right)$ for all $I \in \mathscr{I}$.

5.3.3 Lemma. - Let $\left(\beta_{A}, \delta_{A}\right)$ be an action of $\mathcal{G}$ on a $C^{*}$-algebra $A$. With the notations of $[5.3 .2$. we have:

1. $q_{\beta_{A} \alpha}=\sum_{I \in \mathscr{I}} n_{I}^{-1} q_{I} \otimes e_{\bar{I}} ;$

2. $\left(q_{I} \otimes 1_{S}\right) \delta_{A}(a)=\left(1_{A} \otimes e_{I}\right) \delta_{A}(a)$, for all $a \in A$ and $I \in \mathscr{I}$;

3. $\delta_{A}(a)\left(q_{I} \otimes 1_{S}\right)=\delta_{A}(a)\left(1_{A} \otimes e_{I}\right)$, for all $a \in A$ and $I \in \mathscr{I}$.

Proof. Statement 1 is just restatement of 8.2.18 By a straightforward computation, we verify that $\left(q_{I} \otimes 1_{S}\right) q_{\beta_{A} \alpha}=\left(1_{A} \otimes e_{I}\right) q_{\beta_{A} \alpha}$ for all $I \in \mathscr{I}$. Statement 2 then follows from the fact that $\delta_{A}\left(1_{A}\right)=q_{\beta_{A} \alpha}$. The last statement follows from the second one by taking the adjoint.

5.3.4 Proposition. - Let $\left(\beta_{A}, \delta_{A}\right)$ be an action of $\mathcal{G}$ on a $C^{*}$-algebra $A$. If $\mathcal{G}$ is semi-regular, the Banach space $\left[\left(\mathrm{id}_{A} \otimes \omega\right) \delta_{A}(a) ; a \in A, \omega \in \mathcal{B}(\mathscr{H})_{*}\right] \subset \mathcal{M}(A)$ is a $C^{*}$-algebra.

Proof. Let us denote $T:=\left[\left(\operatorname{id}_{A} \otimes \omega\right) \delta_{A}(a) ; a \in A, \omega \in \mathcal{B}(\mathscr{H})_{*}\right]$. For all $a \in A$ and $\omega \in \mathcal{B}(\mathscr{H})_{*}$, we have $\left(\operatorname{id}_{A} \otimes \omega\right)\left(\delta_{A}(a)\right)^{*}=\left(\operatorname{id}_{A} \otimes \bar{\omega}\right) \delta_{A}\left(a^{*}\right)$. Hence, $T^{*} \subset T$. Let us prove that $T T \subset T$. Let $\omega, \phi \in \mathcal{B}(\mathscr{H})_{*}, a, b \in A$ and $x, y \in \mathcal{K}$. We have

$$
\left(\mathrm{id}_{A} \otimes y \omega\right) \delta_{A}(a)\left(\mathrm{id}_{A} \otimes \phi x\right) \delta_{A}(b)=\left(\mathrm{id}_{A} \otimes \phi \otimes \omega\right)\left(\delta_{A}(a)_{13}\left(1_{A} \otimes x \otimes y\right) \delta_{A}(b)_{12}\right) .
$$

By $5.3 .31,2$, we have

$$
\begin{aligned}
\delta_{A}(a)_{13}\left(1_{A} \otimes x \otimes y\right) \delta_{A}(b)_{12} & =\sum_{I \in \mathscr{I}} n_{I}^{-1} \delta_{A}(a)_{13}\left(1_{A} \otimes x \otimes e_{\bar{I}} y\right)\left(q_{I} \otimes 1 \otimes 1\right) \delta_{A}(b)_{12} \\
& =\sum_{I \in \mathscr{I}} n_{I}^{-1} \delta_{A}(a)_{13}\left(1_{A} \otimes x e_{I} \otimes e_{\bar{I}} y\right) \delta_{A}(b)_{12} \\
& =\delta_{A}(a)_{13}\left((x \otimes 1) q_{\alpha}(1 \otimes y)\right)_{23} \delta_{A}(b)_{12} .
\end{aligned}
$$


It follows from $4 \cdot 7$ that $\left(\operatorname{id}_{A} \otimes y \omega\right) \delta_{A}(a)\left(\operatorname{id}_{A} \otimes \phi x\right) \delta_{A}(b)$ is the norm limit of finite sums of elements of the form

$$
\begin{aligned}
c & :=\left(\operatorname{id}_{A} \otimes \phi \otimes \omega\right)\left(\delta_{A}(a)_{13}\left(\left(x^{\prime} \otimes 1\right) W\left(1 \otimes y^{\prime}\right)\right)_{23} \delta_{A}(b)_{12}\right) \\
& =\left(\operatorname{id}_{A} \otimes \phi x^{\prime} \otimes y^{\prime} \omega\right)\left(\delta_{A}(a)_{13} W_{23} \delta_{A}(b)_{12}\right),
\end{aligned}
$$

where $x^{\prime}, y^{\prime} \in \mathcal{K}$. By combining the following formulas;

$$
\delta_{A}^{2}(a)=W_{23}^{*} \delta_{A}(a)_{13} W_{23} ; \quad W W^{*}=q_{\alpha \widehat{\beta}} ; \quad\left[\delta_{A}(a)_{13}, q_{\alpha \widehat{\beta}, 23}\right]=0\left(\text { since } \widehat{\beta}\left(N^{\mathrm{o}}\right) \subset M^{\prime}\right) ;
$$

we obtain $\delta_{A}(a)_{13} W_{23}=W_{23} \delta_{A}^{2}(a)$. Hence, we have $c=\left(\operatorname{id}_{A} \otimes \psi\right)\left(\delta_{A}^{2}(a)_{13} \delta_{A}(b)_{12}\right)$, where $\psi:=\left(\phi x^{\prime} \otimes y \omega\right) W \in \mathcal{B}(\mathscr{H} \otimes \mathscr{H})_{*}$. Therefore, $c$ is the norm limit of finite sums of elements of the form $\left(\operatorname{id}_{A} \otimes \phi^{\prime} \otimes \omega^{\prime}\right)\left(\delta_{A}^{2}(a)_{13} \delta_{A}(b)_{12}\right)=\left(\mathrm{id} \otimes \phi^{\prime}\right) \delta_{A}\left(\left(\mathrm{id}_{A} \otimes \omega^{\prime}\right) \delta_{A}(a) b\right)$, where $\phi^{\prime}, \omega^{\prime} \in \mathcal{B}(\mathscr{H})_{*}$. Hence, $\left(\operatorname{id}_{A} \otimes y \omega\right) \delta_{A}(a)\left(\mathrm{id}_{A} \otimes \phi x\right) \delta_{A}(b) \in T$.

5.3.5 Definition. - Let $\left(\beta_{A}, \delta_{A}\right)$ be an action of $\mathcal{G}$ on a $C^{*}$-algebra $A$. We say that $\left(\beta_{A}, \delta_{A}\right)$ is weakly continuous if we have $A=\left[\left(\operatorname{id}_{A} \otimes \omega\right) \delta_{A}(a) ; a \in A, \omega \in \mathcal{B}(\mathscr{H})_{*}\right]$.

Note that any strongly continuous action $\left(\beta_{A}, \delta_{A}\right)$ of $\mathcal{G}$ on a $C^{*}$-algebra $A$ is necessarily continuous in the weak sense. Indeed, if $\left(\beta_{A}, \delta_{A}\right)$ is strongly continuous we have the inclusion $\delta_{A}(A)\left(1_{A} \otimes S\right) \subset A \otimes S$. Hence, $\left[\left(\operatorname{id}_{A} \otimes \omega\right) \delta_{A}(a) ; a \in A, \omega \in \mathcal{B}(\mathscr{H})_{*}\right] \subset A$ since $S \subset \mathcal{B}(\mathscr{H})$ is non-degenerate. Conversely, let $\omega \in \mathcal{B}(\mathscr{H})_{*}$ such that $\omega \circ \alpha=\epsilon$. We have $\left(\operatorname{id}_{A} \otimes \omega\right)\left(q_{\beta_{A} \alpha}\right)=1_{A}$. By writing $\omega=y \omega^{\prime}$ for some $\omega^{\prime} \in \mathcal{B}(\mathscr{H})_{*}$ and $y \in S$, we obtain $\left(\operatorname{id}_{A} \otimes \omega^{\prime}\right)\left(q_{\beta_{A} \alpha}(a \otimes y)\right)=a$ for all $a \in A$.

5.3.6 THEOREM. - If the groupoid $\mathcal{G}$ is regular, then any weakly continuous action of $\mathcal{G}$ is strongly continuous.

Proof. Let us fix an action $\left(\beta_{A}, \delta_{A}\right)$ of $\mathcal{G}$ on a $C^{*}$-algebra $A$. Let us assume that $\left(\beta_{A}, \delta_{A}\right)$ is weakly continuous. Since $W \in \mathcal{M}(S \otimes \mathcal{K})$ is a partial isometry such that $W^{*} W=q_{\beta \alpha}$, we have $(S \otimes \mathcal{K}) W=(S \otimes \mathcal{K}) q_{\beta \alpha}$. We recall that $\delta_{A}(a)_{13} W_{23}=W_{23} \delta_{A}^{2}(a)$ for all $a \in A$ (cf. proof of 5.3.4). By 5.1.5 1 , we have $q_{\beta \alpha, 23} \delta_{A}^{2}(a)=\delta_{A}^{2}(a)$ for all $a \in A$. By combining the assertions of the above discussion with 5.3 .33 and 4.81 , we have

$$
\begin{aligned}
(A & \otimes S) q_{\beta_{A} \alpha}=\left[\left(\left(\operatorname{id}_{A} \otimes \omega\right) \delta_{A}(a) \otimes y\right) q_{\beta_{A} \alpha} ; a \in A, y \in S, \omega \in \mathcal{B}(\mathscr{H})_{*}\right] \\
& =\left[\left(\left(\operatorname{id}_{A} \otimes \mathrm{id}_{S} \otimes x \omega\right)\left(\delta_{A}(a)_{13}\left(1_{A} \otimes y \otimes 1_{S}\right) q_{\beta_{A} \alpha, 12}\right) ; a \in A, x \in \mathcal{K}, y \in S, \omega \in \mathcal{B}(\mathscr{H})_{*}\right]\right. \\
& =\left[\left(\operatorname{id}_{A} \otimes \mathrm{id}_{S} \otimes \omega\right)\left(\delta_{A}(a)_{13}\left(\left(y \otimes 1_{\mathcal{K}}\right) q_{\alpha}\left(1_{S} \otimes x\right)\right)_{23}\right) ; a \in A, x \in \mathcal{K}, y \in S, \omega \in \mathcal{B}(\mathscr{H})_{*}\right] \\
& =\left[\left(\operatorname{id}_{A} \otimes \mathrm{id}_{S} \otimes \omega\right)\left(\delta_{A}(a)_{13}\left(\left(y \otimes 1_{\mathcal{K}}\right) W\left(1_{S} \otimes x\right)\right)_{23}\right) ; a \in A, x \in \mathcal{K}, y \in S, \omega \in \mathcal{B}(\mathscr{H})_{*}\right] \\
& =\left[\left(\operatorname{id}_{A} \otimes \mathrm{id}_{S} \otimes \omega\right)\left(\left(1_{A} \otimes y \otimes 1\right) \delta_{A}(a)_{13} W_{23}\right) ; a \in A, x \in \mathcal{K}, y \in S, \omega \in \mathcal{B}(\mathscr{H})_{*}\right] \\
& =\left[\left(\operatorname{id}_{A} \otimes \mathrm{id}_{S} \otimes \omega\right)\left(\left(1_{A} \otimes(y \otimes x) W\right) \delta_{A}^{2}(a)\right) ; a \in A, x \in \mathcal{K}, y \in S, \omega \in \mathcal{B}(\mathscr{H})_{*}\right] \\
& =\left[\left(\operatorname{id}_{A} \otimes \operatorname{id}_{S} \otimes \omega\right)\left(\left(1_{A} \otimes y \otimes x\right) \delta_{A}^{2}(a)\right) ; a \in A, x \in \mathcal{K}, y \in S, \omega \in \mathcal{B}(\mathscr{H})_{*}\right] \\
& =\left[\left(1_{A} \otimes y\right) \delta_{A}\left(\left(\operatorname{id}_{A} \otimes \omega x\right) \delta_{A}(a)\right) ; a \in A, x \in \mathcal{K}, y \in S, \omega \in \mathcal{B}(\mathscr{H})_{*}\right] \\
& =\left[\left(1_{A} \otimes S\right) \delta_{A}(A)\right] .
\end{aligned}
$$

As a first application, we have the result below.

5.3.7 Proposition. - If the groupoid $\mathcal{G}$ is regular, then the trivial action of $\mathcal{G}$ on $N^{0}$ (cf. 5.1.4) is strongly continuous and there exists a unique $\widehat{\mathcal{G}}$-equivariant ${ }^{*}$-isomorphism $\tau: N^{0} \rtimes \mathcal{G} \rightarrow S$ such that $\tau\left(\pi\left(n^{\mathrm{o}}\right) \widehat{\theta}(x)\right)=\beta\left(n^{\mathrm{o}}\right) x$ for all $n \in N$ and $x \in \widehat{S}$.

Proof. In this proof, we use the notations of 5.3.2 with $A:=N^{\circ}$. In this case, we have $q_{I}=\varepsilon_{I}^{\mathrm{o}}$ for all $I \in \mathscr{I}$. According to Theorem 5.3.6, it suffices to show that the trivial action is weakly continuous. Since the $C^{*}$-algebra $N$ is finite-dimensional, it amounts to proving that $\varepsilon_{I}^{\mathrm{o}} \in\left\langle\left(\operatorname{id}_{N^{\circ}} \otimes \omega\right) \delta_{N^{\circ}}\left(n^{\mathrm{o}}\right) ; n \in N, \omega \in \mathcal{B}(\mathscr{H})_{*}\right\rangle$ for all $I \in \mathscr{I}$. Let $I \in \mathscr{I}$. For all $n^{\prime} \in N$, there exists $\omega \in \mathcal{B}(\mathscr{H})_{*}$ such that $\omega(\alpha(n))=\epsilon\left(n^{\prime} n\right)$ for all $n \in N$ (extension of normal linear forms). In particular, there exists $\omega \in \mathcal{B}(\mathscr{H})_{*}$ such that $\omega\left(\alpha\left(\varepsilon_{J}\right)\right)=n_{I} \delta_{J}^{I}$ for all $J \in \mathscr{I}$. By a straightforward computation, we have $\varepsilon_{I}^{\mathrm{o}}=\left(\mathrm{id}_{N^{\circ}} \otimes \omega\right) \delta_{N^{\circ}}\left(1_{N^{\circ}}\right)$ and the weak continuity of the trivial action is then proved since $N^{\mathrm{o}}$ is unital. Since $N^{\mathrm{o}}$ is unital, we have $\widehat{\theta}(x)=\pi\left(1_{N^{\circ}}\right) \widehat{\theta}(x) \in N^{o} \rtimes \mathcal{G}$ for all $x \in \widehat{S}$. Moreover, we have $\pi\left(n^{\mathrm{o}}\right) \widehat{\theta}(x)=\widehat{\theta}\left(\beta\left(n^{\mathrm{o}}\right) x\right)$ for all $n \in N$ and $x \in \widehat{S}$. Hence, the morphism $\widehat{\theta}$ induces a ${ }^{*}$-isomorphism $\widehat{S} \simeq N^{\mathrm{o}} \rtimes \mathcal{G}$. The equivariance is easily obtained from the definitions. 


\section{NOTION OF EQUIVARIANT HILBERT C*-MODULES}

\subsection{Actions of measured quantum groupoids on Hilbert $C^{*}$-modules}

In this section, we introduce a notion of $\mathcal{G}$-equivariant Hilbert $\mathrm{C}^{*}$-module for a measured quantum groupoid $\mathcal{G}$ on a finite basis in the spirit of [3]. We fix a measured quantum groupoid $\mathcal{G}$ on a finite-dimensional basis $N=\bigoplus_{1 \leqslant l \leqslant k} \mathrm{M}_{n_{l}}(\mathbb{C})$ endowed with the nonnormalized Markov trace $\epsilon=\bigoplus_{1 \leqslant l \leqslant k} n_{l} \cdot \operatorname{Tr}_{l}$. We use all the notations introduced in $\S \S 3.1$. 3.2. Let us fix a $\mathcal{G}$ - $C^{*}$-algebra $A$.

THE THREE PICTURES. Following $\$ 2[3]$, an action of $\mathcal{G}$ on a Hilbert $A$-module $\mathscr{E}$ will be defined by three equivalent data:

- a pair $\left(\beta_{\mathscr{E}}, \delta_{\mathscr{E}}\right)$ consisting of a ${ }^{*}$-homomorphism $\beta_{\mathscr{E}}: N^{\mathrm{O}} \rightarrow \mathcal{L}(\mathscr{E})$ and a linear map $\delta_{\mathscr{E}}: \mathscr{E} \rightarrow \widetilde{\mathcal{M}}(\mathscr{E} \otimes S)$, cf. 6.1.1.

- a pair $\left(\beta_{\mathscr{E}}, \mathcal{V}_{\mathscr{E}}\right)$ consisting of a ${ }^{*}$-homomorphism $\beta_{\mathscr{E}}: N^{\mathrm{O}} \rightarrow \mathcal{L}(\mathscr{E})$ and an isometry $\mathcal{V} \in \mathcal{L}\left(\mathscr{E} \otimes_{\delta_{A}}(A \otimes S), \mathscr{E} \otimes S\right)$, cf. 6.1.4

- an action $\left(\beta_{J}, \delta_{J}\right)$ of $\mathcal{G}$ on $J:=\mathcal{K}(\mathscr{E} \oplus A)$, cf.6.1.8.

satisfying some conditions.

We have the following unitary equivalences of Hilbert modules:

$$
\begin{aligned}
& A \otimes_{\delta_{A}}(A \otimes S) \rightarrow q_{\beta_{A} \alpha}(A \otimes S) ; a \otimes_{\delta_{A}} x \mapsto \delta_{A}(a) x ; \\
& (A \otimes S) \otimes_{\delta_{A} \otimes \mathrm{id}_{S}}(A \otimes S \otimes S) \rightarrow q_{\beta_{A} \alpha, 12}(A \otimes S \otimes S) ; x \otimes_{\delta_{A} \otimes \mathrm{id}_{S}} y \mapsto\left(\delta_{A} \otimes \mathrm{id}_{S}\right)(x) y ; \\
& (A \otimes S) \otimes_{\operatorname{id}_{A} \otimes \delta}(A \otimes S \otimes S) \rightarrow q_{\beta \alpha, 23}(A \otimes S \otimes S) ; x \otimes_{\mathrm{id}_{A} \otimes \delta} y \mapsto\left(\operatorname{id}_{A} \otimes \delta\right)(x) y .
\end{aligned}
$$

In the following, we fix a Hilbert $A$-module $\mathscr{E}$. We will apply the usual identifications $\mathcal{M}(A \otimes S)=\mathcal{L}(A \otimes S), \mathcal{K}(\mathscr{E}) \otimes S=\mathcal{K}(\mathscr{E} \otimes S)$ and $\mathcal{M}(\mathcal{K}(\mathscr{E}) \otimes S)=\mathcal{L}(\mathscr{E} \otimes S)$.

6.1.1 Definition. - An action of $\mathcal{G}$ on the Hilbert $A$-module $\mathscr{E}$ is a pair $\left(\beta_{\mathscr{E}}, \delta_{\mathscr{E}}\right)$, where $\beta_{\mathscr{E}}: N^{\mathrm{o}} \rightarrow \mathcal{L}(\mathscr{E})$ is a non-degenerate ${ }^{*}$-homomorphism and $\delta_{\mathscr{E}}: \mathscr{E} \rightarrow \widetilde{\mathcal{M}}(\mathscr{E} \otimes S)$ is a linear map such that:

1. for all $a \in A$ and $\xi, \eta \in \mathscr{E}$, we have

$$
\delta_{\mathscr{E}}(\xi a)=\delta_{\mathscr{E}}(\xi) \delta_{A}(a) \quad \text { and } \quad\left\langle\delta_{\mathscr{E}}(\xi), \delta_{\mathscr{E}}(\eta)\right\rangle=\delta_{A}(\langle\xi, \eta\rangle)
$$

2. $\left[\delta_{\mathscr{E}}(\mathscr{E})(A \otimes S)\right]=q_{\beta_{\mathscr{E}} \alpha}(\mathscr{E} \otimes S)$;

3. for all $\xi \in \mathscr{E}$ and $n \in N$, we have $\delta_{\mathscr{E}}\left(\beta_{\mathscr{E}}\left(n^{\mathrm{o}}\right) \xi\right)=\left(1_{\mathscr{E}} \otimes \beta\left(n^{\mathrm{o}}\right)\right) \delta_{\mathscr{E}}(\xi)$;

4. the linear maps $\delta_{\mathscr{E}} \otimes \mathrm{id}_{S}$ and $\mathrm{id}_{\mathscr{E}} \otimes \delta$ extend to linear maps from $\mathcal{L}(A \otimes S, \mathscr{E} \otimes S)$ to $\mathcal{L}(A \otimes S \otimes S, \mathscr{E} \otimes S \otimes S)$ and we have

$$
\left(\delta_{\mathscr{E}} \otimes \operatorname{id}_{S}\right) \delta_{\mathscr{E}}(\xi)=\left(\operatorname{id}_{\mathscr{E}} \otimes \delta\right) \delta_{\mathscr{E}}(\xi) \in \mathcal{L}(A \otimes S \otimes S, \mathscr{E} \otimes S \otimes S), \quad \text { for all } \xi \in \mathscr{E} .
$$

6.1.2 REMARKs. - - If the second formula of the condition 1 holds, then $\delta_{\mathscr{E}}$ is isometric (cf. [4], 8.3.2 1).

- If the condition 1 holds, then the condition 2 is equivalent to:

$$
\left[\delta_{\mathscr{E}}(\mathscr{E})\left(1_{A} \otimes S\right)\right]=q_{\beta_{\mathscr{E}} \alpha}(\mathscr{E} \otimes S) .
$$

Indeed, if $\left(u_{\lambda}\right)_{\lambda}$ is an approximate unit of $A$ we have

$$
\delta_{\mathscr{E}}(\xi)=\lim _{\lambda} \delta_{\mathscr{E}}\left(\xi u_{\lambda}\right)=\lim _{\lambda} \delta_{\mathscr{E}}(\xi) \delta_{A}\left(u_{\lambda}\right)=\delta_{\mathscr{E}}(\xi) q_{\beta_{A}{ }^{\alpha},} \quad \text { for all } \xi \in \mathscr{E} .
$$

By strong continuity of the action $\left(\beta_{A}, \delta_{A}\right)$, the condition 1 of Definition 6.1.1 and the equality $\mathscr{E} A=\mathscr{E}$, we then have $\left[\delta_{\mathscr{E}}(\mathscr{E})(A \otimes S)\right]=\left[\delta_{\mathscr{E}}(\mathscr{E})\left(1_{A} \otimes S\right)\right]$ and the equivalence follows. 
- Note that we have $q_{\beta_{\mathscr{E}} \alpha} \delta_{\mathscr{E}}(\xi)=\delta_{\mathscr{E}}(\xi)=\delta_{\mathscr{E}}(\xi) q_{\beta_{A} \alpha}$ for all $\xi \in \mathscr{E}$.

- We will prove (cf. 6.1.7) that if $\delta_{\mathscr{E}}$ satisfies the conditions 1 and 2 of 6.1.1. then the extensions of $\delta_{\mathscr{E}} \otimes \mathrm{id}_{\mathcal{S}}$ and $\mathrm{id}_{\mathscr{E}} \otimes \delta$ always exist and satisfy the formulas:

$$
\begin{aligned}
\left(\mathrm{id}_{\mathscr{E}} \otimes \delta\right)(T)\left(\mathrm{id}_{A} \otimes \delta\right)(x) & =\left(\mathrm{id}_{\mathscr{E}} \otimes \delta\right)(T x) ; \\
\left(\delta_{\mathscr{E}} \otimes \mathrm{id}_{S}\right)(T)\left(\delta_{A} \otimes \mathrm{id}_{S}\right)(x) & =\left(\delta_{\mathscr{E}} \otimes \operatorname{id}_{S}\right)(T x) ;
\end{aligned}
$$

for all $x \in A \otimes S$ and $T \in \mathcal{L}(A \otimes S, \mathscr{E} \otimes S)$.

6.1.3 Notation. - For $\xi \in \mathscr{E}$, let us denote by $T_{\xi} \in \mathcal{L}\left(A \otimes S, \mathscr{E} \otimes_{\delta_{A}}(A \otimes S)\right)$ the operator defined by

$$
T_{\xi}(x):=\xi \otimes_{\delta_{A}} x, \quad \text { for all } x \in A \otimes S .
$$

We have $T_{\mathcal{\zeta}}^{*}\left(\eta \otimes_{\delta_{A}} y\right)=\delta_{A}(\langle\xi, \eta\rangle) y$ for all $\eta \in \mathscr{E}$ and $y \in A \otimes S$. In particular, we have $T_{\xi}^{*} T_{\eta}=\delta_{A}(\langle\xi, \eta\rangle)$ for all $\xi, \eta \in \mathscr{E}$.

6.1.4 Definition. - Let $\mathcal{V} \in \mathcal{L}\left(\mathscr{E} \otimes_{\delta_{A}}(A \otimes S), \mathscr{E} \otimes S\right)$ be an isometry and $\beta_{\mathscr{E}}: N^{\mathrm{o}} \rightarrow \mathcal{L}(\mathscr{E})$ a non-degenerate ${ }^{*}$-homomorphism such that:

1. $\mathscr{V}^{\mathcal{V}^{*}}=q_{\beta_{\S} \alpha} ;$

2. $\mathcal{V}\left(\beta_{\mathscr{E}}\left(n^{\mathrm{o}}\right) \otimes_{\delta_{A}} 1\right)=\left(1_{\mathscr{E}} \otimes \beta\left(n^{\mathrm{o}}\right)\right) \mathcal{V}$, for all $n \in N$.

Then, $\mathcal{V}$ is said to be admissible if we further have:

3. $\mathcal{V} T_{\xi} \in \widetilde{\mathcal{M}}(\mathscr{E} \otimes S)$, for all $\xi \in \mathscr{E}$;

4. $\left(\mathcal{V} \otimes_{\mathcal{C}} \operatorname{id}_{S}\right)\left(\mathcal{V} \otimes_{\delta_{A} \otimes i_{S}} 1\right)=\mathcal{V} \otimes_{\mathrm{id}_{A} \otimes \delta} 1 \in \mathcal{L}\left(\mathscr{E} \otimes_{\delta_{A}^{2}}(A \otimes S \otimes S), \mathscr{E} \otimes S \otimes S\right)$.

The fourth statement in the previous definition makes sense since we have used the canonical identifications thereafter. By combining the associativity of the internal tensor product with the unitary equivalences $(6.2)$ and $(6.3)$, we obtain the following unitary equivalences of Hilbert $A \otimes S$-modules:

$$
\begin{aligned}
\left(\mathscr{E} \otimes_{\delta_{A}}(A \otimes S)\right) \otimes_{\delta_{A} \otimes \mathrm{id}_{S}}(A \otimes S \otimes S) & \rightarrow \mathscr{E} \otimes_{\delta_{A}^{2}}(A \otimes S \otimes S) \\
\left(\xi \otimes_{\delta_{A}} x\right) \otimes_{\delta_{A} \otimes \mathrm{id}_{S}} y & \mapsto \xi \otimes_{\delta_{A}^{2}}\left(\delta_{A} \otimes \mathrm{id}_{S}\right)(x) y ; \\
\left(\mathscr{E} \otimes_{\delta_{A}}(A \otimes S)\right) \otimes_{\mathrm{id}_{A} \otimes \delta}(A \otimes S \otimes S) & \rightarrow \mathscr{E} \otimes_{\delta_{A}^{2}}(A \otimes S \otimes S) \\
\left(\xi \otimes_{\delta_{A}} x\right) \otimes_{\mathrm{id}_{A} \otimes \delta} y & \mapsto \xi \otimes_{\delta_{A}^{2}}\left(\mathrm{id}_{A} \otimes \delta\right)(x) y .
\end{aligned}
$$

We also have the following:

$$
\begin{aligned}
(\mathscr{E} \otimes S) \otimes_{\delta_{A} \otimes \mathrm{id}_{S}}(A \otimes S \otimes S) & \rightarrow\left(\mathscr{E} \otimes_{\delta_{A}}(A \otimes S)\right) \otimes S \\
(\xi \otimes S) \otimes_{\delta_{A} \otimes \mathrm{id}_{S}}(x \otimes t) & \mapsto\left(\xi \otimes_{\delta_{A}} x\right) \otimes s t ; \\
(\mathscr{E} \otimes S) \otimes_{\mathrm{id}_{A} \otimes \delta}(A \otimes S \otimes S) & \rightarrow q_{\beta \alpha, 23}(\mathscr{E} \otimes S \otimes S) \subset \mathscr{E} \otimes S \otimes S \\
\xi \otimes_{\mathrm{id}_{A} \otimes \delta} y & \mapsto\left(\mathrm{id}_{\mathscr{E}} \otimes \delta\right)(\xi) y .
\end{aligned}
$$

In particular, $\mathcal{V} \otimes_{\delta_{A} \otimes \mathrm{id}_{S}} 1 \in \mathcal{L}\left(\mathscr{E} \otimes_{\delta_{A}^{2}}(A \otimes S \otimes S),(\mathscr{E} \otimes S) \otimes_{\delta_{A} \otimes \mathrm{id}_{S}}(A \otimes S \otimes S)\right)$ (6.4) and $\mathcal{V} \otimes_{\mathrm{C}} \operatorname{id}_{S} \in \mathcal{L}\left((\mathscr{E} \otimes S) \otimes_{\delta_{A} \otimes \operatorname{id}_{S}}(A \otimes S \otimes S), \mathscr{E} \otimes S \otimes S\right)$ 6.6.

The next result provides an equivalence of the definitions 6.1.1 and 6.1.4

6.1.5 Proposition. - a) Let $\delta_{\mathscr{E}}: \mathscr{E} \rightarrow \widetilde{\mathcal{M}}(\mathscr{E} \otimes S)$ be a linear map and $\beta_{\mathscr{E}}: N^{\mathrm{o}} \rightarrow \mathcal{L}(\mathscr{E}) a$ non-degenerate *-homomorphism which satisfy the conditions 1,2 , and 3 of Definition 6.1.1 Then, there exists a unique isometry $\mathcal{V} \in \mathcal{L}\left(\mathscr{E} \otimes_{\delta_{A}}(A \otimes S), \mathscr{E} \otimes S\right)$ such that $\delta_{\mathscr{E}}(\xi)=\mathcal{V} T_{\xi}$ for all $\xi \in \mathscr{E}$. Moreover, the pair $\left(\beta_{\mathscr{E}}, \mathcal{V}\right)$ satisfies the conditions 1, 2, and 3 of Definition 6.1.4 
b) Conversely, let $\mathcal{V} \in \mathcal{L}\left(\mathscr{E} \otimes_{\delta_{A}}(A \otimes S), \mathscr{E} \otimes S\right)$ be an isometry and $\beta_{\mathscr{E}}: N^{\mathrm{o}} \rightarrow \mathcal{L}(\mathscr{E})$ a non-degenerate *-homomorphism, which satisfy the conditions 1, 2, and 3 of Definition 6.1 .4 Let us consider the map $\delta_{\mathscr{E}}: \mathscr{E} \rightarrow \mathcal{L}(A \otimes S, \mathscr{E} \otimes S)$ given by $\delta_{\mathscr{E}}(\xi):=\mathcal{V} T_{\xi}$ for all $\xi \in \mathscr{E}$. Then, the pair $\left(\beta_{\mathscr{E}}, \delta_{\mathscr{E}}\right)$ satisfies the conditions 1, 2 and 3 of Definition 6.1.1

c) Let us assume that the above statements hold. Then, the pair $\left(\beta_{\mathscr{E}}, \delta_{\mathscr{E}}\right)$ is an action of $\mathcal{G}$ on $\mathscr{E}$ if, and only if, $\mathcal{V}$ is admissible.

In the proof, we will use the following notation.

6.1.6 Notation. - Let $\mathcal{E}$ and $\mathcal{F}$ be Hilbert $C^{*}$-modules. Let $q \in \mathcal{L}(\mathcal{E})$ be a self-adjoint projection and $T \in \mathcal{L}(q \mathcal{E}, \mathcal{F})$. Let $\widetilde{T}: \mathcal{E} \rightarrow \mathcal{F}$ be the map defined by $\widetilde{T} \xi:=T q \xi$, for all $\xi \in \mathcal{E}$. Therefore, $\widetilde{T} \in \mathcal{L}(\mathcal{E}, \mathcal{F})$ and $\widetilde{T}^{*}=q T^{*}$. By abuse of notation, we will still denote by $T$ the adjointable operator $\widetilde{T}$.

Proof of Proposition 6.1.5 a) By definition of the internal tensor product and 6.1.1 1 , there exists a unique isometric $(A \otimes S)$-linear map $\mathcal{V}: \mathscr{E} \otimes \delta_{A}(A \otimes S) \rightarrow \mathscr{E} \otimes S$ such that

$$
\mathcal{V}\left(\xi \otimes_{\delta_{A}} x\right)=\delta_{\mathscr{E}}(\xi) x, \quad \text { for all } \xi \in \mathscr{E} \text { and } x \in A \otimes S .
$$

In other words, we have $\mathcal{V} T_{\xi}=\delta_{\mathscr{E}}(\xi)$ for all $\xi \in \mathscr{E}$. Now, it follows from 6.1.1 2 that the ranges of $\mathcal{V}$ and $q_{\beta_{\mathscr{E}} \alpha}$ are equal. Then, denote by $v$ the range restriction of $\mathcal{V}$. Hence, the map $v^{-1} q_{\beta_{\mathscr{E}} \alpha}$ is an adjoint for $\mathcal{V}$. Indeed, for all $x \in \mathscr{E} \otimes S$ and $y \in \mathscr{E} \otimes \delta_{A}(A \otimes S)$ we have

$$
\begin{aligned}
& \left\langle v^{-1} q_{\beta_{\mathscr{E}} \alpha} x, y\right\rangle=\left\langle\mathfrak{V} v^{-1}\left(q_{\beta_{\mathscr{E}} \alpha} x\right), \mathfrak{V} y\right\rangle \\
& (\mathscr{V} \text { is isometric) } \\
& =\left\langle q_{\beta_{\mathscr{E}} \alpha} x, \mathscr{V} y\right\rangle \\
& =\langle x, \mathcal{V} y\rangle-\left\langle\left(1-q_{\beta_{\mathscr{E}} \alpha}\right)(x), \mathcal{V} y\right\rangle \\
& =\langle x, \mathcal{V} y\rangle \text {. } \\
& \left(\mathcal{V} y \in \operatorname{im}\left(q_{\beta_{\mathscr{E}} \alpha}\right)\right)
\end{aligned}
$$

Hence, $\mathcal{V} \in \mathcal{L}\left(\mathscr{E} \otimes \delta_{A}(A \otimes S), \mathscr{E} \otimes S\right)$ and then $\mathcal{V}^{*} \mathcal{V}=1$ and $\mathcal{V} \mathcal{V}^{*}=\mathscr{V} v^{-1} q_{\beta_{\mathscr{E}} \alpha}=q_{\beta_{\mathscr{E}} \alpha}$. The conditions 1 and 3 of Definition 6.1.4 are then fulfilled. Now, we have

$$
\begin{aligned}
\mathcal{V}\left(\beta_{\mathscr{E}}\left(n^{\mathrm{o}}\right) \otimes_{\delta_{A}} 1\right)\left(\xi \otimes_{\delta_{A}} x\right) & =\delta_{\mathscr{E}}\left(\beta_{\mathscr{E}}\left(n^{\mathrm{o}}\right) \xi\right) x \\
& =\left(1_{\mathscr{E}} \otimes \beta\left(n^{\mathrm{o}}\right)\right) \delta_{\mathscr{E}}(\xi) x \\
& =\left(1_{\mathscr{E}} \otimes \beta\left(n^{\mathrm{o}}\right)\right) \mathcal{V}\left(\xi \otimes_{\delta_{A}} x\right),
\end{aligned}
$$

for all $\xi \in \mathscr{E}, x \in A \otimes S$ and $n \in N$. Hence, the condition 2 of Definition 6.1.4 holds.

$b)$ is straightforward.

c) Let $T \in \mathcal{L}(A \otimes S, \mathscr{E} \otimes S)$. By using 6.1.6 and the identifications 6.3. 6.7), we have $T \otimes_{\mathrm{id}_{A} \otimes \delta} 1 \in \mathcal{L}(A \otimes S \otimes S, \mathscr{E} \otimes S \otimes S)$. Now, we can define the extension

$$
\mathrm{id}_{\mathscr{E}} \otimes \delta: \mathcal{L}(A \otimes S, \mathscr{E} \otimes S) \rightarrow \mathcal{L}(A \otimes S \otimes S, \mathscr{E} \otimes S \otimes S)
$$

by setting

$$
\left(\mathrm{id}_{\mathscr{E}} \otimes \delta\right)(T):=T \otimes_{\mathrm{id}_{A} \otimes \delta} 1, \quad \text { for all } T \in \mathcal{L}(A \otimes S, \mathscr{E} \otimes S) .
$$

We also have $T \otimes_{\delta_{A} \otimes \mathrm{id}_{S}} 1 \in \mathcal{L}\left(A \otimes S \otimes S,\left(\mathscr{E} \otimes \delta_{A}(A \otimes S)\right) \otimes S\right)$ by using again 6.1.6 and the identifications $6.2,6.6$. Let us define the extension

$$
\delta_{\mathscr{E}} \otimes \mathrm{id}_{S}: \mathcal{L}(A \otimes S, \mathscr{E} \otimes S) \rightarrow \mathcal{L}(A \otimes S \otimes S, \mathscr{E} \otimes S \otimes S)
$$

by setting

$$
\left(\delta_{\mathscr{E}} \otimes \mathrm{id}_{S}\right)(T):=\left(\mathcal{V} \otimes_{\mathcal{C}} 1_{S}\right)\left(T \otimes_{\delta_{A} \otimes \mathrm{id}_{S}} 1\right), \quad \text { for all } T \in \mathcal{L}(A \otimes S, \mathscr{E} \otimes S) .
$$

Therefore, for all $\xi \in \mathscr{E}$ we have:

$$
\begin{aligned}
& \left(\delta_{\mathscr{E}} \otimes \mathrm{id}_{S}\right) \delta_{\mathscr{E}}(\xi)=\left(\mathcal{V} \otimes_{\mathbb{C}} 1_{S}\right)\left(\mathcal{V} \otimes_{\delta_{A} \otimes \mathrm{id}_{S}} 1\right)\left(T_{\xi} \otimes_{\delta_{A} \otimes \mathrm{id}_{S}} 1\right) \in \mathcal{L}(A \otimes S \otimes S, \mathscr{E} \otimes S \otimes S) \\
& \left(\mathrm{id}_{\mathscr{E}} \otimes \delta\right) \delta_{\mathscr{E}}(\xi)=\left(\mathcal{V} \otimes_{\mathrm{id}_{A} \otimes \delta} 1\right)\left(T_{\xi} \otimes_{\mathrm{id}_{A} \otimes \delta} 1\right) \in \mathcal{L}(A \otimes S \otimes S, \mathscr{E} \otimes S \otimes S)
\end{aligned}
$$


where:

$$
\begin{gathered}
T_{\xi} \otimes_{\delta_{A} \otimes \mathrm{id}_{S}} 1 \in \mathcal{L}\left(A \otimes S \otimes S, \mathscr{E} \otimes_{\delta_{A}^{2}}(A \otimes S \otimes S)\right) ; \\
T_{\xi} \otimes_{\mathrm{id}_{A} \otimes \delta} 1 \in \mathcal{L}\left(A \otimes S \otimes S, \mathscr{E} \otimes_{\delta_{A}^{2}}(A \otimes S \otimes S)\right) ;
\end{gathered}
$$

by using the identifications 6.2, 6.4 and 6.3.6.5) respectively and 6.1.6. In particular, if $\mathcal{V}$ is admissible, then the condition 4 of Definition 6.1.1 holds.

Conversely, let us assume that the above condition is satisfied. In order to show that $\mathcal{V}$ is admissible, we only have to prove that the restrictions of the operators $T_{\xi} \otimes_{\delta_{A} \otimes \mathrm{id}_{S}} 1$ and $T_{\xi} \otimes_{\mathrm{id}_{A} \otimes \delta} 1$ to the Hilbert submodule $q_{\beta_{A} \alpha, 12} q_{\beta \alpha, 23}(A \otimes S \otimes S)$ are surjective.

Let $a \in A, x \in A \otimes S$ and $y \in A \otimes S \otimes S$. Let us set $z=\left(\delta_{A} \otimes \operatorname{id}_{S}\right)\left(\delta_{A}(a) x\right) y$. It is clear that $z \in q_{\beta_{A} \alpha, 12} q_{\beta \alpha, 23}(A \otimes S \otimes S)$. By a straightforward computation, we have

$$
\left(T_{\xi} \otimes_{\delta_{A} \otimes \mathrm{id}_{S}} 1\right)(z)=\xi a \otimes_{\delta_{A}^{2}}\left(\delta_{A} \otimes \mathrm{id}_{S}\right)(x) y .
$$

Hence, the restriction of $T_{\xi} \otimes_{\delta_{A} \otimes \mathrm{id}_{S}} 1$ to $q_{\beta_{A} \alpha, 12} q_{\beta \alpha, 23}(A \otimes S \otimes S)$ is surjective in virtue of (6.4) and the fact that $\mathscr{E} A=\mathscr{E}$. The same statement is also true for $T_{\xi} \otimes_{\mathrm{id}_{A} \otimes \delta} 1$.

6.1.7 Remarks. - In the proof of Proposition 6.1.5. we have proved the statements below.

- By applying 6.1.6 and the identifications 6.3. 6.7), we have obtained a linear map $\mathrm{id}_{\mathscr{E}} \otimes \delta: \mathcal{L}(A \otimes S, \mathscr{E} \otimes S) \rightarrow \mathcal{L}(A \otimes S \otimes S, \mathscr{E} \otimes S \otimes S)$ given by

$$
\left(\mathrm{id}_{\mathscr{E}} \otimes \delta\right)(T):=T \otimes_{\mathrm{id}_{A} \otimes \delta} 1, \quad \text { for all } T \in \mathcal{L}(A \otimes S, \mathscr{E} \otimes S) ;
$$

- If $\delta_{\mathscr{E}}$ satisfies the conditions 1 and 2 of Definition 6.1.1. let $\mathcal{V}$ be the isometry associated with $\delta_{\mathscr{E}}$ (cf.6.1.5 a)). By applying 6.1.6 and the identifications 6.2, 6.6), the linear $\operatorname{map} \delta_{\mathscr{E}} \otimes \mathrm{id}_{S}: \mathcal{L}(A \otimes S, \mathscr{E} \otimes S) \rightarrow \mathcal{L}(A \otimes S \otimes S, \mathscr{E} \otimes S \otimes S)$ is defined by

$$
\left(\delta_{\mathscr{E}} \otimes \mathrm{id}_{S}\right)(T):=\left(\mathcal{V} \otimes_{\mathcal{C}} 1_{S}\right)\left(T \otimes_{\delta_{A} \otimes \mathrm{id}_{S}} 1\right), \quad \text { for all } T \in \mathcal{L}(A \otimes S, \mathscr{E} \otimes S) .
$$

Note that the extensions $\mathrm{id}_{\mathscr{E}} \otimes \delta$ and $\delta_{\mathscr{E}} \otimes \mathrm{id}_{S}$ satisfy the following formulas:

$\left(\mathrm{id}_{\mathscr{E}} \otimes \delta\right)(T)\left(\mathrm{id}_{A} \otimes \delta\right)(x)=\left(\operatorname{id}_{\mathscr{E}} \otimes \delta\right)(T x) ;\left(\delta_{\mathscr{E}} \otimes \operatorname{id}_{S}\right)(T)\left(\delta_{A} \otimes \mathrm{id}_{S}\right)(x)=\left(\delta_{\mathscr{E}} \otimes \mathrm{id}_{S}\right)(T x)$

for all $x \in A \otimes S$ and $T \in \mathcal{L}(A \otimes S, \mathscr{E} \otimes S)$.

Let us denote by $J:=\mathcal{K}(\mathscr{E} \oplus A)$ the linking $C^{*}$-algebra associated with the Hilbert $A$ module $\mathscr{E}$. In the following, we apply the usual identifications $\mathcal{M}(J)=\mathcal{L}(\mathscr{E} \oplus A)$ and $\mathcal{M}(J \otimes S)=\mathcal{L}((\mathscr{E} \otimes S) \oplus(A \otimes S))$.

6.1.8 Definition. - An action $\left(\beta_{J}, \delta_{J}\right)$ of $\mathcal{G}$ on $J$ is said to be compatible with the action $\left(\beta_{A}, \delta_{A}\right)$ if:

1. $\delta_{J}: J \rightarrow \mathcal{M}(J \otimes S)$ is compatible with $\delta_{A}$, i.e. $\iota_{A \otimes S} \circ \delta_{A}=\delta_{J} \circ \iota_{A}$;

2. $\beta_{J}: N^{\mathrm{o}} \rightarrow \mathcal{M}(J)$ is compatible with $\beta_{A}$, i.e. $\iota_{A}\left(\beta_{A}\left(n^{\mathrm{o}}\right) a\right)=\beta_{J}\left(n^{\mathrm{o}}\right) \iota_{A}(a)$, for all $n \in N$ and $a \in A$.

6.1.9 Proposition. - Let $\left(\beta_{J}, \delta_{J}\right)$ be a compatible action of $\mathcal{G}$ on J. There exists a unique non-degenerate ${ }^{*}$-homomorphism $\beta_{\mathscr{E}}: N^{\mathrm{o}} \rightarrow \mathcal{L}(\mathscr{E})$ such that

$$
\beta_{J}\left(n^{\mathrm{o}}\right)=\left(\begin{array}{cc}
\beta_{\mathscr{E}}\left(n^{\mathrm{o}}\right) & 0 \\
0 & \beta_{A}\left(n^{\mathrm{o}}\right)
\end{array}\right), \quad \text { for all } n \in N \text {. }
$$

Moreover, we have $q_{\beta_{J} \alpha}=\left(\begin{array}{cc}q_{\beta_{\&} \alpha} & 0 \\ 0 & q_{\beta_{A} \alpha}\end{array}\right)$. 
Proof. Note that since $\iota_{A}, \beta_{A}$ and $\beta_{J}$ are ${ }^{*}$-homomorphisms, the condition 2 of Definition 6.1.8 is equivalent to:

$$
\iota_{A}\left(a \beta_{A}\left(n^{\mathrm{o}}\right)\right)=\iota_{A}(a) \beta_{J}\left(n^{\mathrm{o}}\right), \quad \text { for all } a \in A, n \in N \text {. }
$$

Therefore, there exists a map $\beta_{\mathscr{E}}: N^{\mathrm{o}} \rightarrow \mathcal{L}(\mathscr{E})$ necessarily unique such that

$$
\beta_{J}\left(n^{\mathrm{o}}\right)=\left(\begin{array}{cc}
\beta_{\mathscr{E}}\left(n^{\mathrm{o}}\right) & 0 \\
0 & \beta_{A}\left(n^{\mathrm{o}}\right)
\end{array}\right),
$$

for all $n \in N$. Then, it is clear that $\beta_{\mathscr{E}}$ is a non-degenerate ${ }^{*}$-homomorphism and the last statement is then an immediate consequence.

6.1.10 Remarks. - Note that if $\beta_{A}$ is injective, then so is $\beta_{J}$. For all $n \in N, \xi \in \mathscr{E}$ and $k \in \mathcal{K}(\mathscr{E})$, we have $\iota_{\mathcal{K}(\mathscr{E})}\left(\beta_{\mathscr{E}}\left(n^{\mathrm{o}}\right) k\right)=\beta_{J}\left(n^{\mathrm{o}}\right) \iota_{\mathcal{K}(\mathscr{E})}(k)$ and $\iota_{\mathscr{E}}\left(\beta_{\mathscr{E}}\left(n^{\mathrm{o}}\right) \xi\right)=\beta_{J}\left(n^{\mathrm{o}}\right) \iota_{\mathscr{E}}(\xi)$. In particular, we have $\beta_{\mathscr{E}}\left(n^{\mathrm{o}}\right) \theta_{\xi, \eta}=\theta_{\beta_{\mathscr{E}}\left(n^{\mathrm{o}}\right) \xi, \eta}$ for all $n \in N$ and $\xi, \eta \in \mathscr{E}$ (cf. 2.3.2 2).

6.1.II Proposition. - a) Let us assume that the $C^{*}$-algebra $J$ is endowed with a compatible action $\left(\beta_{J}, \delta_{J}\right)$ of $\mathcal{G}$ such that $\delta_{J}(J) \subset \widetilde{\mathcal{M}}(J \otimes S)$. Then, we have the following statements:

- there exists a unique linear map $\delta_{\mathscr{E}}: \mathscr{E} \rightarrow \widetilde{\mathcal{M}}(\mathscr{E} \otimes S)$ such that $\iota_{\mathscr{E} \otimes S} \circ \delta_{\mathscr{E}}=\delta_{J} \circ \iota_{\mathscr{E}}$; furthermore, the pair $\left(\beta_{\mathscr{E}}, \delta_{\mathscr{E}}\right)$ is an action of $\mathcal{G}$ on $\mathscr{E}$, where $\beta_{\mathscr{E}}: N^{\mathrm{O}} \rightarrow \mathcal{L}(\mathscr{E})$ is the *-homomorphism defined in 6.1.9.

- there exists a unique faithful *-homomorphism $\delta_{\mathcal{K}(\mathscr{E})}: \mathcal{K}(\mathscr{E}) \rightarrow \widetilde{\mathcal{M}}(\mathcal{K}(\mathscr{E}) \otimes S)$ such that $\iota_{\mathcal{K}(\mathscr{E} \otimes S)} \circ \delta_{\mathcal{K}(\mathscr{E})}=\delta_{J} \circ \iota_{\mathcal{K}(\mathscr{E})} ;$ moreover, the pair $\left(\beta_{\mathscr{E}}, \delta_{\mathcal{K}(\mathscr{E})}\right)$ is an action of $\mathcal{G}$ on $\mathcal{K}(\mathscr{E})$.

b) Conversely, let $\left(\beta_{\mathscr{E}}, \delta_{\mathscr{E}}\right)$ be an action of $\mathcal{G}$ on the Hilbert $A$-module $\mathscr{E}$. Then, there exists a faithful *-homomorphism $\delta_{J}: J \rightarrow \widetilde{\mathcal{M}}(J \otimes S)$ such that $\iota_{\mathscr{E} \otimes S} \circ \delta_{\mathscr{E}}=\delta_{J} \circ \iota_{\mathscr{E}}$. Moreover, we define a unique action $\left(\beta_{J}, \delta_{J}\right)$ of $\mathcal{G}$ on $J$ compatible with $\left(\beta_{A}, \delta_{A}\right)$ by setting

$$
\beta_{J}\left(n^{\mathrm{o}}\right)=\left(\begin{array}{cc}
\beta_{\mathscr{E}}\left(n^{\mathrm{o}}\right) & 0 \\
0 & \beta_{A}\left(n^{\mathrm{o}}\right)
\end{array}\right), \quad \text { for all } n \in N \text {. }
$$

Proof. a) Let us assume that the $C^{*}$-algebra $J$ is endowed with a compatible action $\left(\beta_{J}, \delta_{J}\right)$ of $\mathcal{G}$. Let $\beta_{\mathscr{E}}: N^{\mathrm{O}} \rightarrow \mathcal{L}(\mathscr{E})$ be the ${ }^{*}$-homomorphism defined in Proposition 6.1.9. By strict continuity and 6.1.8 1 , we have $\delta_{J}\left(\iota_{A}(m)\right)=\iota_{A \otimes S}\left(\delta_{A}(m)\right)$ for all $m \in \mathcal{M}(A)$. It then follows from 6.1 .9 that

$$
\delta_{J}\left(\iota_{\mathcal{K}(\mathscr{E})}\left(1_{\mathscr{E}}\right)\right)=\delta_{J}\left(1_{J}\right)-\delta_{J}\left(\iota_{A}\left(1_{A}\right)\right)=q_{\beta_{J} \alpha}-\iota_{A \otimes S}\left(q_{\beta_{A} \alpha}\right)=\iota_{\mathcal{K}(\mathscr{E} \otimes S)}\left(q_{\beta_{\mathscr{E}} \alpha}\right) .
$$

Let $\xi \in \mathscr{E}$. We have $\iota_{\mathcal{K}(\mathscr{E})}\left(1_{\mathscr{E}}\right) \iota_{\mathscr{E}}(\xi)=\iota_{\mathscr{E}}(\xi)$ and $\iota_{\mathscr{E}}(\xi) \iota_{\mathcal{K}(\mathscr{E})}\left(1_{\mathscr{E}}\right)=0$. Hence,

$$
\iota_{\mathcal{K}(\mathscr{E} \otimes S)}\left(q_{\beta_{\mathscr{E}} \alpha}\right) \delta_{J}\left(\iota_{\mathscr{E}}(\xi)\right)=\delta_{J}\left(\iota_{\mathscr{E}}(\xi)\right) \quad \text { and } \quad \delta_{J}\left(\iota_{\mathscr{E}}(\xi)\right) \iota_{\mathcal{K}(\mathscr{E} \otimes S)}\left(q_{\beta_{\mathscr{E}} \alpha}\right)=0 .
$$

We have $\iota_{A \otimes S}(x) \delta_{J}\left(\iota_{\mathscr{E}}(\xi)\right)=\iota_{A \otimes S}(x) \iota_{\mathcal{L}(\mathscr{E} \otimes S)}\left(q_{\beta_{\mathscr{E}} \alpha}\right) \delta_{J}\left(\iota_{\mathscr{E}}(\xi)\right)=0$, for all $x \in A \otimes S$. Now, let $\left(u_{\lambda}\right)_{\lambda}$ be an approximate unit of $A$. We have

$$
\delta_{J}\left(\iota_{\mathscr{E}}(\xi)\right)=\lim _{\lambda} \delta_{J}\left(\iota_{\mathscr{E}}\left(\xi u_{\lambda}\right)\right)=\lim _{\lambda} \delta_{J}\left(\iota_{\mathscr{E}}(\xi)\right) \iota_{A \otimes S}\left(\delta_{A}\left(u_{\lambda}\right)\right) .
$$

Hence, $\delta_{J}\left(\iota_{\mathscr{E}}(\xi)\right) \iota_{\mathscr{E} \otimes S}(\eta)=0$ for all $\eta \in \mathscr{E} \otimes S$. Hence, there exists a unique linear map $\delta_{\mathscr{E}}: \mathscr{E} \rightarrow \mathcal{L}(A \otimes S, \mathscr{E} \otimes S)$ such that $\iota_{\mathscr{E} \otimes S} \circ \delta_{\mathscr{E}}=\delta_{J} \circ \iota_{\mathscr{E}}$ (cf. 2.3.4 I). Moreover, $\delta_{\mathscr{E}}$ actually takes its values in the subspace $\widetilde{\mathcal{M}}(\mathscr{E} \otimes S)$ of $\mathcal{L}(A \otimes S, \mathscr{E} \otimes S)$. Indeed, let us fix $\xi \in \mathscr{E}$ and $s \in S$. By assumption, we have that

$$
\iota_{\mathscr{E} \otimes S}\left(\left(1_{\mathscr{E}} \otimes s\right) \delta_{\mathscr{E}}(\xi)\right)=\left(1_{J} \otimes s\right) \delta_{J}\left(\iota_{\mathscr{E}}(\xi)\right) \text { and } \iota_{\mathscr{E} \otimes S}\left(\delta_{\mathscr{E}}(\xi)\left(1_{A} \otimes s\right)\right)=\delta_{J}\left(\iota_{\mathscr{E}}(\xi)\right)\left(1_{J} \otimes s\right)
$$

belong to $J \otimes S=\mathcal{K}((\mathscr{E} \otimes S) \oplus(A \otimes S))$. It then follows that $\left(1_{\mathscr{E}} \otimes s\right) \delta_{\mathscr{E}}(\xi) \in \mathscr{E} \otimes S$ and $\delta_{\mathscr{E}}(\xi)\left(1_{A} \otimes s\right) \in \mathscr{E} \otimes S$. The first condition of 6.1.1 is easily derived from the compatibility of $\delta_{J}$. The vector subspace of $\delta_{J}\left(1_{J}\right)((\mathscr{E} \oplus A) \otimes S)$ spanned by

$$
\left\{\delta_{J}\left(\theta_{\xi \oplus a, \eta \oplus b}\right)(\zeta) ; \xi, \eta \in \mathscr{E}, a, b \in A, \zeta \in(\mathscr{E} \oplus A) \otimes S\right\}
$$


is dense. However, we have

$$
\delta_{J}\left(\theta_{\xi \oplus a, \eta \oplus b}\right)(\zeta)=\left(\delta_{\mathscr{E}}(\xi) \oplus \delta_{A}(a)\right)\left(\delta_{\mathscr{E}}(\eta) \oplus \delta_{A}(b)\right)^{*}(\zeta),
$$

where $\delta_{\mathscr{E}}(\xi) \oplus \delta_{A}(a), \delta_{\mathscr{E}}(\eta) \oplus \delta_{A}(b) \in \mathcal{L}(A \otimes S, \mathscr{E} \otimes S) \oplus \mathcal{L}(A \otimes S) \subset \mathcal{L}(A \otimes S,(\mathscr{E} \oplus A) \otimes S)$. In particular, the vector subspace of $\delta_{J}\left(1_{J}\right)((\mathscr{E} \oplus A) \otimes S)$ spanned by

$$
\left\{\delta_{\mathscr{E}}(\xi) x \oplus \delta_{A}(a) x ; \xi \in \mathscr{E}, a \in A, x \in A \otimes S\right\}
$$

is dense. Therefore, the relation $\left[\delta_{\mathscr{E}}(\mathscr{E})(A \otimes S)\right]=q_{\beta_{\mathscr{E}} \alpha}(\mathscr{E} \otimes S)$ follows since we also have $\delta_{J}\left(1_{J}\right)((\mathscr{E} \oplus A) \otimes S)=q_{\beta_{\mathscr{E}} \alpha}(\mathscr{E} \otimes S) \oplus q_{\beta_{A} \alpha}(A \otimes S)$.

Let us fix $\xi \in \mathscr{E}$ and $n \in N$. We have

$$
\begin{aligned}
\iota_{\mathscr{E} \otimes S}\left(\delta_{\mathscr{E}}\left(\beta_{\mathscr{E}}\left(n^{\mathrm{o}}\right) \xi\right)\right)=\delta_{J}\left(\iota_{\mathscr{E}}\left(\beta_{\mathscr{E}}\left(n^{\mathrm{o}}\right) \xi\right)\right)=\delta_{J}\left(\beta_{J}\left(n^{\mathrm{o}}\right) \iota_{\mathscr{E}}(\xi)\right) & =\left(1_{J} \otimes \beta\left(n^{\mathrm{o}}\right)\right) \delta_{J}\left(\iota_{\mathscr{E}}(\xi)\right) \\
& =\iota_{\mathscr{E} \otimes S}\left(\left(1_{\mathscr{E}} \otimes \beta\left(n^{\mathrm{o}}\right) \delta_{\mathscr{E}}(\xi)\right) .\right.
\end{aligned}
$$

Hence, $\delta_{\mathscr{E}}\left(\beta_{\mathscr{E}}\left(n^{\mathrm{o}}\right) \xi\right)=\left(1_{\mathscr{E}} \otimes \beta\left(n^{\mathrm{o}}\right)\right) \delta_{\mathscr{E}}(\xi)$.

Let us consider the linear maps $\mathrm{id}_{\mathscr{E}} \otimes \delta: \mathcal{L}(A \otimes S, \mathscr{E} \otimes S) \rightarrow \mathcal{L}(A \otimes S \otimes S, \mathscr{E} \otimes S \otimes S)$ and $\delta_{\mathscr{E}} \otimes \mathrm{id}_{S}: \mathcal{L}(A \otimes S, \mathscr{E} \otimes S) \rightarrow \mathcal{L}(A \otimes S \otimes S, \mathscr{E} \otimes S \otimes S)$ (cf.6.1.7). By using (6.8) and the compatibility of $\delta_{J}$ with $\delta_{A}$ and $\delta_{\mathscr{E}}$, it follows from a straightforward computation that

$\iota_{\mathscr{E} \otimes S} \otimes S\left(\left(\mathrm{id}_{\mathscr{E}} \otimes \delta\right)(T)\right)=\left(\mathrm{id}_{J} \otimes \delta\right)\left(\iota_{\mathscr{E} \otimes S}(T)\right) ; \iota_{\mathscr{E} \otimes S \otimes S}\left(\left(\delta_{\mathscr{E}} \otimes \mathrm{id}_{S}\right)(T)\right)=\left(\delta_{J} \otimes \mathrm{id}_{S}\right)\left(\iota_{\mathscr{E} \otimes S}(T)\right) ;$

for all $T \in \mathcal{L}(A \otimes S, \mathscr{E} \otimes S)$. In particular, $\iota_{\mathscr{E} \otimes S} \otimes S\left(\left(\mathrm{id}_{\mathscr{E}} \otimes \delta\right) \delta_{\mathscr{E}}(\xi)\right)=\left(\operatorname{id}_{J} \otimes \delta\right) \delta_{J}\left(\iota_{\mathscr{E}}(\xi)\right)$ and $\iota_{\mathscr{E} \otimes S} \otimes S\left(\left(\delta_{\mathscr{E}} \otimes \mathrm{id}_{S}\right) \delta_{\mathscr{E}}(\xi)\right)=\left(\delta_{J} \otimes \mathrm{id}_{S}\right) \delta_{J}\left(\iota_{\mathscr{E}}(\xi)\right)$ for all $\xi \in \mathscr{E}$. Hence, for all $\xi \in \mathscr{E}$ we have $\left(\delta_{\mathscr{E}} \otimes \mathrm{id}_{S}\right) \delta_{\mathscr{E}}(\xi)=\left(\mathrm{id}_{\mathscr{E}} \otimes \delta\right) \delta_{\mathscr{E}}(\xi)$. Therefore, the pair $\left(\beta_{\mathscr{E}}, \delta_{\mathscr{E}}\right)$ is an action of $\mathcal{G}$ on $\mathscr{E}$.

We claim that there exists a unique *-homomorphism $\delta_{\mathcal{K}(\mathscr{E})}: \mathcal{K}(\mathscr{E}) \rightarrow \mathcal{M}(\mathcal{K}(\mathscr{E}) \otimes S)$ such that $\iota_{\mathcal{K}(\mathscr{Q} \otimes S)} \circ \delta_{\mathcal{K}(\mathscr{E})}=\delta_{J} \circ \iota_{\mathcal{K}(\mathscr{E})}$. We recall that $\delta_{J}\left(\iota_{\mathcal{K}(\mathscr{E})}\left(1_{\mathscr{E}}\right)\right)=\iota_{\mathcal{K}(\mathscr{E} \otimes S)}\left(q_{\beta_{\mathscr{E}} \alpha}\right)$. We also have $\iota_{A \otimes S}(x) \iota_{\mathcal{K}(\mathscr{E} \otimes S)}\left(q_{\beta_{\mathscr{E}} \alpha}\right)=0$ and $\iota_{\mathcal{K}(\mathscr{E} \otimes S)}\left(q_{\beta_{\mathscr{E}} \alpha}\right) \iota_{A \otimes S}(x)=0$ for all $x \in A \otimes S$. It follows that $\iota_{A \otimes S}(x) \delta_{J}\left(\iota_{\mathcal{K}(\mathscr{E})}(k)\right)=0$ and $\delta_{J}\left(\iota_{\mathcal{K}(\mathscr{E})}(k)\right) \iota_{A \otimes S}(x)=0$ for all $k \in \mathcal{K}(\mathscr{E})$ and $x \in A \otimes S$. Hence, $\delta_{J}\left(\iota_{\mathcal{K}(\mathscr{E})}(k)\right) \in \iota_{\mathcal{K}(\mathscr{E} \otimes S)}(\mathcal{L}(\mathscr{E} \otimes S))$ (cf. 2.3.4) and the claim is proved since $\iota_{\mathcal{K}(\mathscr{E} \otimes S)}$ is faithful. Since $\iota_{\mathcal{K}(\mathscr{E} \otimes S)}$ is isometric and $\delta_{J} \circ \iota_{\mathcal{K}(\mathscr{E})}$ is strictly continuous, the ${ }^{*}$-homomorphism $\delta_{\mathcal{K}(\mathscr{E})}$ is strictly continuous and extend uniquely to a strictly continuous *-homomorphism $\delta_{\mathcal{K}(\mathscr{E})}: \mathcal{L}(\mathscr{E}) \rightarrow \mathcal{M}(\mathcal{K}(\mathscr{E}) \otimes S)$ such that $\delta_{\mathcal{K}(\mathscr{E})}\left(1_{\mathscr{E}}\right)=q_{\beta_{\mathscr{E}} \alpha}$. Moreover, for all $\xi, \eta \in \mathscr{E}$ we have (cf. 2.3 .2 2)

$$
\delta_{\mathcal{K}(\mathscr{E})}\left(\theta_{\tilde{\zeta}, \eta}\right)=\delta_{\mathscr{E}}(\xi) \circ \delta_{\mathscr{E}}(\eta)^{*}=\theta_{\delta_{\mathscr{E}}(\xi), \delta_{\mathscr{E}}(\eta)} \in \mathcal{K}(\widetilde{\mathcal{M}}(\mathscr{E} \otimes S)) \subset \widetilde{\mathcal{M}}(\mathcal{K}(\mathscr{E}) \otimes S)
$$

Hence, $\delta_{\mathcal{K}(\mathscr{E})}(\mathcal{K}(\mathscr{E})) \subset \widetilde{\mathcal{M}}(\mathcal{K}(\mathscr{E}) \otimes S)$. We have $\delta_{\mathcal{K}(\mathscr{E})}\left(\beta_{\mathscr{E}}\left(n^{\mathrm{o}}\right)\right)=\left(1_{\mathscr{E}} \otimes \beta\left(n^{\mathrm{o}}\right)\right) q_{\beta_{\mathscr{E}} \alpha}$, for all $n \in N$ (cf. 6.9. 6.1.10. By strict continuity, we have the formulas:

$$
\begin{aligned}
& \iota_{\mathcal{K}(\mathscr{E} \otimes S \otimes S)}\left(\mathrm{id}_{\mathcal{K}(\mathscr{E})} \otimes \delta\right)(T)=\left(\mathrm{id}_{J} \otimes \delta\right)\left(\iota_{\mathcal{K}(\mathscr{E} \otimes S)}(T)\right) ; \\
& \left.\iota_{\mathcal{K}(\mathscr{E} \otimes S \otimes S)}\left(\delta_{\mathcal{K}(\mathscr{E})} \otimes \mathrm{id}_{S}\right)(m)\right)=\left(\delta_{J} \otimes \mathrm{id}_{S}\right)\left(\iota_{\mathcal{K}(\mathscr{E} \otimes S)}(T)\right) ;
\end{aligned}
$$

for all $T \in \mathcal{M}(\mathcal{K}(\mathscr{E}) \otimes S)=\mathcal{L}(\mathscr{E} \otimes S)$. By applying the above formulas to $T:=\delta_{\mathcal{K}(\mathscr{E})}(k)$ for $k \in \mathcal{K}(\mathscr{E})$, we show that $\left(\delta_{\mathcal{K}(\mathscr{E})} \otimes \mathrm{id}_{S}\right) \delta_{\mathcal{K}(\mathscr{E})}(k)=\left(\operatorname{id}_{\mathcal{K}(\mathscr{E})} \otimes \delta\right) \delta_{\mathcal{K}(\mathscr{E})}(k)$.

b) First, it is clear that $\beta_{J}$ is a non-degenerate ${ }^{*}$-homomorphism. It is also clear that $\beta_{J}$ is compatible with the fibration map $\beta_{A}$, i.e. $\beta_{J}\left(n^{\mathrm{o}}\right) \iota_{A}(a)=\iota_{A}\left(\beta_{A}\left(n^{\mathrm{o}}\right) a\right)$, for all $a \in A$ and $n \in N$. Let $\mathcal{V} \in \mathcal{L}\left(\mathscr{E} \otimes \delta_{A}(A \otimes S), \mathscr{E} \otimes S\right)$ be the isometry associated with the action $\delta_{\mathscr{E}}$. Let $i: q_{\beta_{A} \alpha}(A \otimes S) \rightarrow A \otimes S$ be the inclusion map. We verify that $i$ is an $(A \otimes S)$-linear adjointable map and $i^{*}=q_{\beta_{A} \alpha}$. In particular, the map $i$ is an isometry since we have $i^{*} i(x)=q_{\beta_{A} \alpha} x=x$ for all $x \in q_{\beta_{A} \alpha}(A \otimes S)$. Let

$$
\mathcal{W}:=\mathcal{V} \oplus i \in \mathcal{L}\left(\left(\mathscr{E} \otimes_{\delta_{A}}(A \otimes S)\right) \oplus q_{\beta_{A} \alpha}(A \otimes S),(\mathscr{E} \otimes S) \oplus(A \otimes S)\right) .
$$

We have $\mathscr{W}^{*} \mathfrak{W}=1$, then $\mathscr{W}$ is an isometry. Henceforth, we will use the following identification (see 6.1)):

$\left(\mathscr{E} \otimes_{\delta_{A}}(A \otimes S)\right) \oplus q_{\beta_{A} \alpha}(A \otimes S)=\left(\mathscr{E} \otimes_{\delta_{A}}(A \otimes S)\right) \oplus\left(A \otimes_{\delta_{A}}(A \otimes S)\right)=(\mathscr{E} \oplus A) \otimes_{\delta_{A}}(A \otimes S)$. 
Hence, $\mathscr{W} \in \mathcal{L}\left((\mathscr{E} \oplus A) \otimes_{\delta_{A}}(A \otimes S),(\mathscr{E} \oplus A) \otimes S\right)$. Let us define

$$
\delta_{J}(x):=\mathscr{W}\left(x \otimes_{\delta_{A}} 1\right)^{2} W^{*} \in \mathcal{M}(J \otimes S), \quad \text { for all } x \in J .
$$

In that way, we define a strictly continuous ${ }^{*}$-homomorphism $\delta_{J}: J \rightarrow \mathcal{M}(J \otimes S)$ satisfying $\delta_{J}\left(1_{J}\right)=\mathscr{W}^{2} \mathscr{W}^{*}=q_{\beta_{\mathscr{E}} \alpha} \oplus q_{\beta_{A} \alpha}=q_{\beta_{J} \alpha}$. Let $a \in A$. Let us prove that $\iota_{A \otimes S}\left(\delta_{A}(a)\right)=\delta_{J}\left(\iota_{A}(a)\right)$. Since $\iota_{A \otimes S}\left(\delta_{A}(a)\right)^{2} \mathscr{W}^{2} \mathscr{W}^{*}=\iota_{A \otimes S}\left(\delta_{A}(a)\right)$ and $\delta_{J}\left(\iota_{A}(a)\right)^{2} \mathscr{W}^{*} W^{*}=\delta_{J}\left(\iota_{A}(a)\right)$, it amounts to proving that $\iota_{A \otimes S}\left(\delta_{A}(a)\right)^{\mathscr{W}}=\delta_{J}\left(\iota_{A}(a)\right)^{\mathscr{W}}$, for all $a \in A$. Therefore, it is enough to prove that $\iota_{A \otimes S}\left(\delta_{A}(a)\right)^{\mathscr{W}}=\mathscr{W}\left(\iota_{A}(a) \otimes_{\delta_{A}} 1\right)$ since $\mathscr{W}^{*} \mathfrak{W}=1$. However, for all $\eta \in \mathscr{E}, b \in A$ and $x \in A \otimes S$ we have

$$
\mathscr{W}\left((\eta \oplus b) \otimes_{\delta_{A}} x\right)=\mathcal{V}\left(\eta \otimes_{\delta_{A}} x\right) \oplus \delta_{A}(b) x=\delta_{\mathscr{E}}(\eta) x \oplus \delta_{A}(b) x .
$$

We finally obtain

$$
\begin{aligned}
\mathscr{W}\left(\iota_{A}(a) \otimes_{\delta_{A}} 1\right)\left((\eta \oplus b) \otimes_{\delta_{A}} x\right) & =\mathscr{W}\left((0 \oplus a b) \otimes_{\delta_{A}} x\right) \\
& =(\mathscr{V} \oplus i)\left(0 \oplus \delta_{A}(a b) x\right) \\
& =0 \oplus \delta_{A}(a) \delta_{A}(b) x \\
& =\iota_{A \otimes S}\left(\delta_{A}(a)\right)\left(\delta_{\mathscr{E}}(\eta) x \oplus \delta_{A}(b) x\right) \\
& =\iota_{A \otimes S}\left(\delta_{A}(a)\right)^{\mathfrak{W}}\left((\eta \oplus b) \otimes_{\delta_{A}} x\right),
\end{aligned}
$$

for all $\eta \in \mathscr{E}, b \in A$ and $x \in A \otimes S$. By using similar arguments, we also prove that $\iota_{\mathscr{E} \otimes S}\left(\delta_{\mathscr{E}}(\xi)\right)=\delta_{J}\left(\iota_{\mathscr{E}}(\xi)\right)$ for all $\xi \in \mathscr{E}$. By strict continuity, we obtain the formulas:

$$
\left(\delta_{J} \otimes \mathrm{id}_{S}\right) \iota_{A \otimes S}(m)=\iota_{A \otimes S \otimes S}\left(\delta_{A} \otimes \mathrm{id}_{S}\right)(m) ;\left(\mathrm{id}_{J} \otimes \delta\right) \iota_{A \otimes S}(m)=\iota_{A \otimes S \otimes S}\left(\mathrm{id}_{A} \otimes \delta\right)(m) ;
$$

for all $m \in \mathcal{M}(A \otimes S)$. By applying the above formulas to $m:=\delta_{A}(a)$ for $a \in A$ and by using again the compatibility of $\delta_{J}$ with $\delta_{A}$, we obtain the formulas:

$\left(\delta_{J} \otimes \mathrm{id}_{S}\right) \delta_{J}\left(\iota_{A}(a)\right)=\iota_{A \otimes S \otimes S}\left(\delta_{A} \otimes \mathrm{id}_{S}\right) \delta_{A}(a) ;\left(\mathrm{id}_{J} \otimes \delta\right) \delta_{J}\left(\iota_{A}(a)\right)=\iota_{A \otimes S \otimes S}\left(\mathrm{id}_{A} \otimes \delta\right) \delta_{A}(a)$.

Hence, $\left(\delta_{J} \otimes \mathrm{id}_{S}\right) \delta_{J}\left(\iota_{A}(a)\right)=\left(\mathrm{id}_{J} \otimes \delta\right) \delta_{J}\left(\iota_{A}(a)\right)$ for all $a \in A$. In a similar way, we have $\left(\delta_{J} \otimes \mathrm{id}_{S}\right) \delta_{J}\left(\iota_{\mathscr{E}}(\xi)\right)=\left(\mathrm{id}_{J} \otimes \delta\right) \delta_{J}\left(\iota_{\mathscr{E}}(\xi)\right)$ for all $\xi \in \mathscr{E}$. However, since $J$ is generated by $\iota_{\mathscr{E}}(\mathscr{E}) \cup \iota_{A}(A)$ as a $C^{*}$-algebra, the coassociativity of $\delta_{J}$ is then proved.

For all $\eta \in \mathscr{E}, b \in A, x \in A \otimes S$ and $n \in N$, we have

$$
\begin{aligned}
\delta_{J}\left(\beta_{J}\left(n^{\mathrm{o}}\right)\right)^{\mathscr{W}}\left((\eta \oplus b) \otimes_{\delta_{A}} x\right) & =\mathscr{W}\left(\beta_{J}\left(n^{\mathrm{o}}\right)(\eta \oplus b) \otimes_{\delta_{A}} x\right) \\
& =\mathscr{W}\left(\left(\beta_{\mathscr{E}}\left(n^{\mathrm{o}}\right) \eta \oplus \beta_{A}\left(n^{\mathrm{o}}\right) b\right) \otimes_{\delta_{A}} x\right) \\
& =\delta_{\mathscr{E}}\left(\beta_{\mathscr{E}}\left(n^{\mathrm{o}}\right) \eta\right) x \oplus \delta_{A}\left(\beta_{A}\left(n^{\mathrm{o}}\right) b\right) x \\
& =\left(1_{J} \otimes \beta\left(n^{\mathrm{o}}\right)\right)\left(\delta_{\mathscr{E}}(\eta) x \oplus \delta_{A}(b) x\right) \\
& =\left(1_{J} \otimes \beta\left(n^{\mathrm{o}}\right)\right)^{\mathscr{W}}\left((\eta \oplus b) \otimes_{\delta_{A}} x\right) .
\end{aligned}
$$

Hence, $\delta_{J}\left(\beta_{J}\left(n^{\mathrm{o}}\right)\right)=\delta_{J}\left(\beta_{J}\left(n^{\mathrm{o}}\right)\right)^{W^{2} W^{*}}=\left(1_{J} \otimes \beta\left(n^{\mathrm{o}}\right)\right)^{W^{2} W^{*}}=\left(1_{J} \otimes \beta\left(n^{\mathrm{o}}\right)\right) \delta_{J}\left(1_{J}\right)$, for all $n \in N$. Therefore, $\left(\beta_{J}, \delta_{J}\right)$ is an action of $\mathcal{G}$ on $J$, compatible with $\left(\beta_{A}, \delta_{A}\right)$.

EQUIVARIANT UNITARY EQUIVALENCE. In this paragraph, we define a notion of equivariance for unitary equivalences of Hilbert modules acted upon by $\mathcal{G}$. We refer the reader to $\$ 8.3$ for the definitions and notations used below.

6.1.12 Definition. - Let $A$ and $B$ be two $\mathcal{G}$ - $C^{*}$-algebras and $\phi: A \rightarrow B$ a $\mathcal{G}$-equivariant *-isomorphism. Let $\mathscr{E}$ and $\mathscr{F}$ be two Hilbert modules over respectively $A$ and $B$ acted upon by $\mathcal{G}$. A $\phi$-compatible unitary operator $\Phi: \mathscr{E} \rightarrow \mathscr{F}$ is said to be $\mathcal{G}$-equivariant if we have

$$
\delta_{\mathscr{F}}(\Phi \xi)=\left(\Phi \otimes \mathrm{id}_{S}\right) \delta_{\mathscr{E}}(\xi), \quad \text { for all } \xi \in \mathscr{E} \text {. }
$$

We recall that the linear map $\Phi \otimes \mathrm{id}_{S}: \mathcal{L}(A \otimes S, \mathscr{E} \otimes S) \rightarrow \mathcal{L}(B \otimes S, \mathscr{F} \otimes S)$ 8.3.6) is the extension of the $\phi \otimes \mathrm{id}_{S}$-compatible unitary operator $\Phi \otimes \mathrm{id}_{S}: \mathscr{E} \otimes S \rightarrow \mathscr{F} \otimes S(8.3 .4)$.

6.1.13 Proposition. - With the notations and hypotheses of 6.1.12, for all $n \in N$ we have $\beta_{\mathscr{F}}\left(n^{\mathrm{o}}\right) \circ \Phi=\Phi \circ \beta_{\mathscr{E}}\left(n^{\mathrm{o}}\right)$. 
Proof. It is clear that $\left(\Phi \otimes \mathrm{id}_{S}\right)\left(\left(1_{\mathscr{E}} \otimes s\right) T\right)=\left(1_{\mathscr{F}} \otimes s\right)\left(\Phi \otimes \mathrm{id}_{S}\right)(T)$ for all $s \in S$ and $T \in \mathcal{L}(A \otimes S, \mathscr{E} \otimes S)$. Let $n \in N$ and $\xi \in \mathscr{E}$. We have $\delta_{\mathscr{F}}\left(\Phi\left(\beta_{\mathscr{E}}\left(n^{\mathrm{o}}\right) \xi\right)\right)=\delta_{\mathscr{F}}\left(\beta_{\mathscr{F}}\left(n^{\mathrm{o}}\right) \Phi \xi\right)$ by 6.1.1 3. Hence, $\Phi\left(\beta_{\mathscr{E}}\left(n^{\mathrm{o}}\right) \xi\right)=\beta_{\mathscr{F}}\left(n^{\mathrm{o}}\right) \Phi \xi$ by 6.1.2 1 .

6.1.14 Definition. - Two Hilbert $C^{*}$-modules $\mathscr{E}$ and $\mathscr{F}$ acted upon by $\mathcal{G}$ are said to be $\mathcal{G}$-equivariantly unitarily equivalent if there exists a $\mathcal{G}$-equivariant unitary operator from $\mathscr{E}$ onto $\mathscr{F}$.

It is clear that the $\mathcal{G}$-equivariant unitary equivalence defines an equivalence relation on the class consisting of the Hilbert $C^{*}$-modules acted upon by $\mathcal{G}$. In the following, we provide equivalent definitions of the $\mathcal{G}$-equivariant unitary equivalence in the two other pictures.

Let $A$ and $B$ be two $\mathcal{G}$ - $C^{*}$-algebras and $\phi: A \rightarrow B$ a $\mathcal{G}$-equivariant ${ }^{*}$-isomomorphism. Let $\mathscr{E}$ and $\mathscr{F}$ be two Hilbert $C^{*}$-modules over $A$ and $B$ respectively and $\Phi: \mathscr{E} \rightarrow \mathscr{F}$ a $\phi$-compatible unitary operator.

6.1.15 LemMA. - The linear map

$$
\mathscr{E} \otimes_{\delta_{A}}(A \otimes S) \rightarrow \mathscr{F} \otimes_{\delta_{B}}(B \otimes S) ; \xi \otimes_{\delta_{A}} x \mapsto \Phi \xi \otimes_{\delta_{B}}\left(\phi \otimes \operatorname{id}_{S}\right)(x)
$$

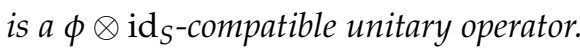

Proof. For all $\xi \in \mathscr{E}, a \in A$ and $x \in A \otimes S$, we have

$$
\begin{aligned}
\Phi(\xi a) \otimes_{\delta_{B}}\left(\phi \otimes \mathrm{id}_{S}\right)(x) & =\Phi(\xi) \phi(a) \otimes_{\delta_{B}}\left(\phi \otimes \mathrm{id}_{S}\right)(x) \\
& =\Phi \xi \otimes_{\delta_{B}} \delta_{B}(\phi(a))\left(\phi \otimes \mathrm{id}_{S}\right)(x) \\
& =\Phi \xi \otimes_{\delta_{B}}\left(\phi \otimes \mathrm{id}_{S}\right)\left(\delta_{A}(a) x\right) .
\end{aligned}
$$

Therefore, we have a well-defined linear map

$$
\Psi: \mathscr{E} \odot_{A}(A \otimes S) \rightarrow \mathscr{F} \otimes_{\delta_{B}}(B \otimes S) ; \xi \odot_{A} x \mapsto \Phi \xi \otimes_{\delta_{B}}\left(\phi \otimes \operatorname{id}_{S}\right)(x) .
$$

For all $\xi, \eta \in \mathscr{E}$, we have $\delta_{B}(\langle\Phi \xi, \Phi \eta\rangle)=\delta_{B}(\phi(\langle\xi, \eta\rangle))=\left(\phi \otimes \operatorname{id}_{S}\right) \delta_{A}(\langle\xi, \eta\rangle)$. Therefore, for all $\xi, \eta \in \mathscr{E}$ and $x, y \in A \otimes S$, we have

$$
\left\langle\Phi \xi \otimes_{\delta_{B}}\left(\phi \otimes \mathrm{id}_{S}\right)(x), \Phi \eta \otimes_{\delta_{B}}\left(\phi \otimes \mathrm{id}_{S}\right)(y)\right\rangle=\left(\phi \otimes \mathrm{id}_{S}\right)\left(\left\langle\xi \otimes_{\delta_{A}} x, \eta \otimes_{\delta_{A}} y\right\rangle\right) .
$$

Hence, $\left\langle\Psi(t), \Psi\left(t^{\prime}\right)\right\rangle=\left(\phi \otimes \operatorname{id}_{S}\right)\left(\left\langle t, t^{\prime}\right\rangle\right)$ for all $t, t^{\prime} \in \mathscr{E} \odot_{A}(A \otimes S)$. In particular, we have $\|\Psi(t)\|=\|t\|$ for all $t \in \mathscr{E} \odot_{A}(A \otimes S)\left(\phi \otimes \mathrm{id}_{S}\right.$ is isometric). Therefore, $\Psi$ extends uniquely to a bounded operator from $\mathscr{E} \otimes_{\delta_{A}}(A \otimes S)$ to $\mathscr{F} \otimes_{\delta_{B}}(B \otimes S)$ still denoted by $\Psi$. We have $\left\langle\Psi(t), \Psi\left(t^{\prime}\right)\right\rangle=\left(\phi \otimes \operatorname{id}_{S}\right)\left(\left\langle t, t^{\prime}\right\rangle\right)$ for all $t, t^{\prime} \in \mathscr{E} \otimes_{\delta_{A}}(A \otimes S)$. Since $\Psi$ is isometric and has a dense range, we conclude that $\Psi$ is surjective. A staightforward computation shows that $\Psi(t x)=\Psi(t)\left(\phi \otimes \operatorname{id}_{S}\right)(x)$ for all $t \in \mathscr{E} \otimes \delta_{A}(A \otimes S)$ and $x \in A \otimes S$.

6.1.16 Proposition. - Let $\left(\beta_{\mathscr{E}}, \delta_{\mathscr{E}}\right)\left(\right.$ resp. $\left.\left(\beta_{\mathscr{F}}, \delta_{\mathscr{F}}\right)\right)$ be an action of $\mathcal{G}$ on $\mathscr{E}$ (resp. FF). Denote by $\mathscr{V}_{\mathscr{E}} \in \mathcal{L}\left(\mathscr{E} \otimes_{\delta_{A}}(A \otimes S), \mathscr{E} \otimes S\right)\left(\right.$ resp. $\left.\mathscr{V}_{\mathscr{F}} \in \mathcal{L}\left(\mathscr{F}_{\mathbb{F}} \otimes_{\delta_{B}}(B \otimes S), \mathscr{F} \otimes S\right)\right)$ the isometry associated with $\left(\beta_{\mathscr{E}}, \delta_{\mathscr{E}}\right)\left(\right.$ resp. $\left.\left(\beta_{\mathscr{F}}, \delta_{\mathscr{F}}\right)\right)$. Assume that $\Phi \circ \beta_{\mathscr{E}}\left(n^{\mathrm{o}}\right)=\beta_{\mathscr{F}}\left(n^{\mathrm{o}}\right) \circ \Phi$ for all $n \in N$. Then, $\Phi$ is $\mathcal{G}$-equivariant if, and only if, we have

$$
\mathcal{V}_{\mathscr{F}}^{*}\left(\Phi \otimes \mathrm{id}_{S}\right) \mathscr{V}_{\mathscr{E}}\left(\xi \otimes_{\delta_{A}} x\right)=\Phi \xi \otimes_{\delta_{B}}\left(\phi \otimes \mathrm{id}_{S}\right)(x),
$$

for all $\xi \in \mathscr{E}$ and $x \in A \otimes S$.

Proof. Let $\Psi: \mathscr{E} \otimes_{\delta_{A}}(A \otimes S) \rightarrow \mathscr{F} \otimes_{\delta_{B}}(B \otimes S)$ be the $\phi \otimes \mathrm{id}_{S}$-compatible unitary operator defined in the above lemma. For all $\xi \in \mathscr{E}$ and $x \in A \otimes S$, we have

$$
\delta_{\mathscr{F}}(\Phi \xi)\left(\phi \otimes \operatorname{id}_{S}\right)(x)=\mathscr{V}_{\mathscr{F}}\left(\Phi \xi \otimes_{\delta_{B}} x\right)=\mathscr{V}_{\mathscr{F}} \circ \Psi\left(\xi \otimes_{\delta_{A}} x\right)
$$

and $\left(\Phi \otimes \mathrm{id}_{S}\right)\left(\delta_{\mathscr{E}}(\xi)\right)\left(\phi \otimes \mathrm{id}_{S}\right)(x)=\left(\Phi \otimes \mathrm{id}_{S}\right)\left(\delta_{\mathscr{E}}(\xi) x\right)=\left(\Phi \otimes \mathrm{id}_{S}\right)^{\mathscr{V}} \mathcal{V}_{\mathscr{E}}\left(\xi \otimes_{\delta_{A}} x\right)$. Therefore, $\delta_{\mathscr{F}} \circ \Phi=\left(\Phi \otimes \mathrm{id}_{S}\right) \circ \delta_{\mathscr{E}}$ if, and only if, $\mathcal{V}_{\mathscr{F}} \circ \Psi=\left(\Phi \otimes \mathrm{id}_{S}\right) \mathcal{V}_{\mathscr{E}}$. In order for the formula $\mathscr{V}_{\mathscr{F}} \circ \Psi=\left(\Phi \otimes \mathrm{id}_{S}\right)^{\mathscr{V}_{\mathscr{E}}}$ to hold true, it is necessary and sufficient that $\Psi=\mathscr{V}_{\mathscr{F}}^{*}\left(\Phi \otimes \mathrm{id}_{S}\right)^{\mathscr{V}_{\mathscr{E}}}$. Indeed, it is necessary since $\mathcal{V}_{\mathscr{F}}^{*} \mathcal{V}_{\mathscr{F}}=1$. It is also sufficient since we have $\mathcal{V}_{\mathscr{F}} \mathcal{V}_{\mathscr{F}}^{*}=q_{\beta_{\mathscr{F}} \alpha}$, $q_{\beta_{\mathscr{F}} \alpha}\left(\Phi \otimes \mathrm{id}_{S}\right)=\left(\Phi \otimes \mathrm{id}_{S}\right) q_{\beta_{\S} \alpha}\left(\right.$ by assumption) and $q_{\beta_{\S} \alpha} \mathcal{V}_{\mathscr{E}}=\mathscr{V}_{\mathscr{E}}$. 
6.1.17 Remark. - Let $A$ be a $\mathcal{G}$-C ${ }^{*}$-algebra. Let $\mathscr{E}$ and $\mathscr{F}$ be two Hilbert $A$-modules acted upon by $\mathcal{G}$. Let $\Phi \in \mathcal{L}(\mathscr{E}, \mathscr{F})$ be a unitary. The following statements are equivalent:

(i) $\Phi$ is $\mathcal{G}$-equivariant;

(ii) $\Phi \circ \beta_{\mathscr{E}}\left(n^{\mathrm{o}}\right)=\beta_{\mathscr{F}}\left(n^{\mathrm{o}}\right) \circ \Phi$ for all $n \in N$ and $\mathcal{V}_{\mathscr{F}}^{*}\left(\Phi \otimes 1_{S}\right) \mathcal{V}_{\mathscr{E}}=\Phi \otimes_{\delta_{A}} 1_{A \otimes S}$;

(iii) $\Phi \circ \beta_{\mathscr{E}}\left(n^{\mathrm{o}}\right)=\beta_{\mathscr{F}}\left(n^{\mathrm{o}}\right) \circ \Phi$ for all $n \in N$ and $\mathcal{V}_{\mathscr{F}}\left(\Phi \otimes \delta_{A} 1_{A \otimes S}\right) \mathcal{V}_{\mathscr{E}}^{*}=q_{\beta_{\mathscr{E}} \alpha}\left(\Phi \otimes 1_{S}\right)$.

6.1.18 Proposition. - Let $A$ and $B$ be two $\mathcal{G}$-C*-algebras and $\phi: A \rightarrow B$ a $\mathcal{G}$-equivariant *-isomorphism. Let $\mathscr{E}$ and $\mathscr{F}$ be two Hilbert modules over respectively $A$ and $B$ acted upon by $\mathcal{G}$. Let $\Phi: \mathscr{E} \rightarrow \mathscr{F}$ be a $\phi$-compatible unitary operator. Denote by $f: \mathcal{K}(\mathscr{E} \oplus A) \rightarrow \mathcal{K}(\mathscr{F} \oplus B)$ the unique *homomorphism such that $f \circ \iota_{\mathscr{E}}=\iota_{\mathscr{F}} \circ T$ and $f \circ \iota_{A}=\iota_{B} \circ \phi(c f$. 8.3.5). Then, $\Phi$ is $\mathcal{G}$-equivariant if, and only if, $f$ is $\mathcal{G}$-equivariant.

Proof. Let $J:=\mathcal{K}(\mathscr{E} \oplus A)$ and $L:=\mathcal{K}(\mathscr{F} \oplus B)$. Assume that $\Phi$ is equivariant. The following formulas are immediate consequences of the definitions:

$$
\begin{aligned}
\iota_{B \otimes S} \circ\left(\phi \otimes \mathrm{id}_{S}\right)(m) & =\left(f \otimes \mathrm{id}_{S}\right) \circ \iota_{A \otimes S}(m), \quad m \in \mathcal{M}(A \otimes S) ; \\
\iota_{\mathscr{F} \otimes S} \circ\left(\Phi \otimes \mathrm{id}_{S}\right)(T) & =\left(f \otimes \mathrm{id}_{S}\right) \circ \iota_{\mathscr{E} \otimes S}(T), \quad T \in \mathcal{L}(A \otimes S, \mathscr{E} \otimes S) .
\end{aligned}
$$

By combining the first (resp. second) formula with the $\mathcal{G}$-equivariance of $\phi$ (resp. $\Phi$ ) and the fact that $f \circ \iota_{A}=\iota_{B} \circ \phi\left(\right.$ resp. $\left.f \circ \iota_{\mathscr{E}}=\iota_{\mathscr{F}} \circ \Phi\right)$, we obtain

$$
\begin{aligned}
& \delta_{L} \circ f\left(\iota_{A}(a)\right)=\left(f \otimes \operatorname{id}_{S}\right) \delta_{J}\left(\iota_{A}(a)\right), \quad \text { for all } a \in A \\
& \left(\operatorname{resp} . \delta_{L} \circ f\left(\iota_{\mathscr{E}}(\xi)\right)=\left(f \otimes \operatorname{id}_{S}\right) \delta_{J}\left(\iota_{\mathscr{E}}(\xi)\right), \quad \text { for all } \xi \in \mathscr{E}\right) \text {. }
\end{aligned}
$$

Therefore, we have $\delta_{L}(f(x))=\left(f \otimes \mathrm{id}_{S}\right) \delta_{J}(x)$ for all $x \in J$. Moreover, by definition of the fibration map on a linking $C^{*}$-algebra (cf. 6.1.11) and the $\mathcal{G}$-equivariance of $\Phi$, we have

$$
f\left(\beta_{J}\left(n^{\mathrm{o}}\right)\right)=\left(\begin{array}{cc}
\beta_{\mathscr{E}}\left(n^{\mathrm{o}}\right) & 0 \\
0 & \beta_{A}\left(n^{\mathrm{o}}\right)
\end{array}\right)=\left(\begin{array}{cc}
\Phi \circ \beta_{\mathscr{E}}\left(n^{\mathrm{o}}\right) \circ \Phi^{-1} & 0 \\
0 & \phi\left(\beta_{A}\left(n^{\mathrm{o}}\right)\right)
\end{array}\right)=\beta_{L}\left(n^{\mathrm{o}}\right),
$$

for all $n \in N$. The converse is proved in a similar way.

CONTINUOUS ACTIONS. In this paragraph, we introduce the notion of continuity for actions of the quantum groupoid $\mathcal{G}$ on Hilbert $A$-modules. If $\mathcal{G}$ is regular, we prove that any action of $\mathcal{G}$ is necessarily continuous.

6.1.19 Definition. - An action $\left(\beta_{\mathscr{E}}, \delta_{\mathscr{E}}\right)$ of $\mathcal{G}$ on a Hilbert $A$-module $\mathscr{E}$ is said to be continuous if we have $\left[\left(1_{\mathscr{E}} \otimes S\right) \delta_{\mathscr{E}}(\mathscr{E})\right]=(\mathscr{E} \otimes S) q_{\beta_{A} \alpha}$. A $\mathcal{G}$-equivariant Hilbert $A$-module is a Hilbert $A$-module $\mathscr{E}$ endowed with a continuous action of $\mathcal{G}$.

6.1.20 Proposition. - Let $\mathscr{E}$ be a $\mathcal{G}$-equivariant Hilbert $A$-module. Let $B:=\mathcal{K}(\mathscr{E})$. We have the following statements:

1. the action $\left(\beta_{B}, \delta_{B}\right)$ of $\mathcal{G}$ on $B$ defined in 6.1 .11 is strongly continuous;

2. we define a continuous action of $\mathcal{G}$ on the Hilbert B-module $\mathscr{E}^{*}$ by setting:

- $\beta_{\mathscr{E}} *\left(n^{\mathrm{o}}\right) T:=\beta_{A}\left(n^{\mathrm{o}}\right) \circ T$, for all $n \in N$ and $T \in \mathscr{E}^{*}$,

- $\delta_{\mathscr{E}}(T) x:=\delta_{\mathscr{E}}\left(T^{*}\right)^{*} \circ x$, for all $T \in \mathscr{E}^{*}$ and $x \in B \otimes S$;

where we have applied the usual identifications $B \otimes S=\mathcal{K}(\mathscr{E} \otimes S)$ and $\mathscr{E}=\mathcal{K}(A, \mathscr{E})$.

Proof. 1. We have $\left[\delta_{B}(B)\left(1_{B} \otimes S\right)\right]=\left[\delta_{B}\left(\theta_{\xi, \eta}\right)\left(1_{B} \otimes y\right) ; \xi, \eta \in \mathscr{E}, y \in S\right]$. However, we have $\delta_{B}\left(\theta_{\xi, \eta}\right)\left(1_{B} \otimes y\right)=\delta_{\mathscr{E}}(\xi) \delta_{\mathscr{E}}(\eta)^{*}\left(1_{B} \otimes y\right)=\delta_{\mathscr{E}}(\xi)\left(\left(1_{B} \otimes y^{*}\right) \delta_{\mathscr{E}}(\eta)\right)^{*}$ for all $y \in S$ and $\xi, \eta \in \mathscr{E}$. It then follows from the continuity of the action $\left(\beta_{\mathscr{E}}, \delta_{\mathscr{E}}\right)$ and 6.1.2 that

$$
\left[\delta_{B}(B)\left(1_{B} \otimes S\right)\right]=\left[\delta_{\mathscr{E}}(\mathscr{E}) q_{\beta_{A} \alpha}\left(\mathscr{E}^{*} \otimes S\right)\right]=\left[\delta_{\mathscr{E}}(\mathscr{E})\left(\mathscr{E}^{*} \otimes S\right)\right] .
$$

Now, by combining the formulas $\left[\delta_{\mathscr{E}}(\mathscr{E})\left(1_{\mathscr{E}} \otimes S\right)\right]=q_{\beta_{\mathscr{E}} \alpha}(\mathscr{E} \otimes S)$ and $B=[\mathscr{E} \mathscr{E} *]$ with the fact that any element of $S$ can be written as a product of two elements of $S$, we obtain $\left[\delta_{B}(B)\left(1_{B} \otimes S\right)\right]=\left[\delta_{\mathscr{E}}(\mathscr{E})\left(1_{\mathscr{E}} \otimes S\right)\left(\mathscr{E}^{*} \otimes S\right)\right]=q_{\beta_{\mathscr{E}} \alpha}(B \otimes S)$.

2. Straightforward. 
6.1.21 Proposition. - Let $\mathscr{E}$ be a Hilbert $A$-module endowed with an action $\left(\beta_{\mathscr{E}}, \delta_{\mathscr{E}}\right)$ of $\mathcal{G}$ on $\mathscr{E}$. Let $J:=\mathcal{K}(\mathscr{E} \oplus A)$ be the associated linking $C^{*}$-algebra. Let $\left(\beta_{J}, \delta_{J}\right)$ be the action defined in 6.1.11 Then, the action $\left(\beta_{\mathscr{E}}, \delta_{\mathscr{E}}\right)$ is continuous if, and only if, the action $\left(\beta_{J}, \delta_{J}\right)$ is strongly continuous.

Proof. Assume that the action $\left(\beta_{\mathscr{E}}, \delta_{\mathscr{E}}\right)$ is continuous. Let $B:=\mathcal{K}(\mathscr{E})$. Let us prove that $\left(\beta_{J}, \delta_{J}\right)$ is strongly continuous. Let $x \in J$ and $s \in S$. Let us write

$$
x=\left(\begin{array}{cc}
b & \xi \\
\eta^{*} & a
\end{array}\right), \quad \text { where } a \in A, b \in B \text { and } \xi, \eta \in \mathscr{E} .
$$

Then, we have

$$
\begin{array}{r}
\delta_{J}(x)\left(1_{J} \otimes s\right)=\iota_{B \otimes S}\left(\delta_{B}(b)\left(1_{B} \otimes s\right)\right)+\iota_{\mathscr{E} \otimes S}\left(\delta_{\mathscr{E}}(\xi)\left(1_{A} \otimes s\right)\right)+\iota_{\mathscr{E} *} \otimes S \\
\left.+\delta_{\mathscr{E} *}\left(\eta^{*}\right)\left(1_{B} \otimes s\right)\right) \\
+\iota_{A \otimes S}\left(\delta_{A}(a)\left(1_{A} \otimes s\right)\right) .
\end{array}
$$

Then, the continuity of $\left(\beta_{J}, \delta_{J}\right)$ follows from the continuity of $\left(\beta_{A}, \delta_{A}\right),\left(\beta_{\mathscr{E}}, \delta_{\mathscr{E}}\right)$ and the continuity of $\left(\beta_{B}, \delta_{B}\right)$ and $\left(\beta_{\mathscr{E} *}, \delta_{\mathscr{E} *}\right)$ 6.1.20. Conversely, assume that $\left(\beta_{J}, \delta_{J}\right)$ is continuous. We have $\iota_{\mathscr{E} \otimes S}\left((\mathscr{E} \otimes S) q_{\beta_{A} \alpha}\right)=\left(\iota_{\mathscr{E}}(\mathscr{E}) \otimes S\right) q_{\beta_{J} \alpha}$. Let $\zeta \in \mathscr{E}$ and $y \in S$. As in the above computation, we prove that $\iota_{\mathscr{E} \otimes S}\left((\zeta \otimes y) q_{\beta_{A} \alpha}\right)$ is the norm limit of finite sums of elements of the following forms: $\iota_{B \otimes S}\left(\left(1_{B} \otimes s\right) \delta_{B}(b)\right), \iota_{\mathscr{E} \otimes S}\left(\left(1_{\mathscr{E}} \otimes s\right) \delta_{\mathscr{E}}(\xi)\right), \iota_{\mathscr{E} *} \otimes S\left(\left(1_{\mathscr{E} *} \otimes s\right) \delta_{\mathscr{E} *}\left(\eta^{*}\right)\right)$ and $\iota_{A \otimes S}\left(\left(1_{A} \otimes S\right) \delta_{A}(a)\right)$. By multiplying by $\iota_{B \otimes S}\left(1_{B \otimes S}\right)$ on the left and by $\iota_{A \otimes S}\left(1_{A \otimes S}\right)$ on the right, we have that $\iota_{\mathscr{E} \otimes S}\left((\zeta \otimes y) q_{\beta_{A} \alpha}\right)$ is the norm limit of finite sums of elements of the form $\iota_{\mathscr{E} \otimes S}\left(\left(1_{\mathscr{E}} \otimes s\right) \delta_{\mathscr{E}}(\xi)\right)$. The continuity of $\left(\beta_{\mathscr{E}}, \delta_{\mathscr{E}}\right)$ follows from the fact that $\iota_{\mathscr{E} \otimes S}$ is isometric.

6.1.22 Definition. - A linking $\mathcal{G}-C^{*}$-algebra is a quintuple $\left(J, \beta_{J}, \delta_{J}, e_{1}, e_{2}\right)$ consisting of a $C^{*}$-algebra $J$ endowed with a continuous action $\left(\beta_{J}, \delta_{J}\right)$ and nonzero self-adjoint projections $e_{1}, e_{2} \in \mathcal{M}(J)$ satisfying the following conditions:

1. $e_{1}+e_{2}=1_{J}$;

2. $\left[J e_{j} J\right]=J$, for all $j=1,2$;

3. $\delta_{J}\left(e_{j}\right)=q_{\beta_{J} \alpha}\left(e_{j} \otimes 1_{S}\right)$, for all $j=1,2$.

6.1.23 Remarks. - $\quad$ - Let $\left(A, \beta_{A}, \delta_{A}\right)$ be a $\mathcal{G}$-C ${ }^{*}$-algebra and $m \in \mathcal{M}(A)$ such that $\delta_{A}(m)=q_{\beta_{A} \alpha}\left(m \otimes 1_{S}\right)$. Let $n \in N$, we have $\left[m, \beta_{A}\left(n^{\mathrm{o}}\right)\right]=0$. Indeed, since $\alpha$ and $\beta$ commute pointwise we have $\left[q_{\beta_{A} \alpha}\left(1_{A} \otimes \beta\left(n^{\mathrm{o}}\right)\right), q_{\beta_{A} \alpha}\left(m \otimes 1_{S}\right)\right]=0$. It then follows that $\delta_{A}\left(\left[m, \beta_{A}\left(n^{\mathrm{o}}\right)\right]\right)=\left[\delta_{A}\left(m \beta_{A}\left(n^{\mathrm{o}}\right)\right), \delta_{A}\left(\beta_{A}\left(n^{\mathrm{o}}\right) m\right)\right]=0$. Hence, $\left[m, \beta_{A}\left(n^{\mathrm{o}}\right)\right]=0$ by faithfulness of $\delta_{A}$. In particular, we have $\left[q_{\beta_{A} \alpha}, m \otimes 1_{S}\right]=0$.

- Let $\left(J, \beta_{J}, \delta_{J}, e_{1}, e_{2}\right)$ be a linking $\mathcal{G}$-C $C^{*}$-algebra. By restriction of the action $\left(\beta_{J}, \delta_{J}\right)$, the corner $e_{2} J e_{2}$ (resp. $e_{1} J e_{2}$ ) turns into a $\mathcal{G}$-C ${ }^{*}$-algebra (resp. $\mathcal{G}$-equivariant Hilbert $\mathrm{C}^{*}$-module over $\left.e_{2} J e_{2}\right)$. Furthermore, we also have the identification of $\mathcal{G}$ - $\mathrm{C}^{*}$-algebras $\mathcal{K}\left(e_{1} J e_{2}\right)=e_{1} J e_{1}$.

- Conversely, if $\left(\mathscr{E}, \beta_{\mathscr{E}}, \delta_{\mathscr{E}}\right)$ is a $\mathcal{G}$-equivariant Hilbert $A$-module, then the $\mathrm{C}^{*}$-algebra $J:=\mathcal{K}(\mathscr{E} \oplus A)$ endowed with the continuous action $\left(\beta_{J}, \delta_{J}\right)$ (cf.6.1.11, 6.1.21) and the projections $e_{1}:=\iota_{\mathscr{E}}\left(1_{\mathscr{E}}\right)$ and $e_{2}:=\iota_{A}\left(1_{A}\right)$ is a linking $\mathcal{G}$ - $C^{*}$-algebra.

6.1.24 LEMMA. - Let $\mathscr{E}$ be a Hilbert $A$-module endowed with an action $\left(\beta_{\mathscr{E}}, \delta_{\mathscr{E}}\right)$ of $\mathcal{G}$. We have $\mathscr{E}=\left[\left(\mathrm{id}_{\mathscr{E}} \otimes \omega\right) \delta_{\mathscr{E}}(\xi) ; \xi \in \mathscr{E}, \omega \in \mathcal{B}(\mathscr{H})_{*}\right](c f .2 .3 .6)$.

Proof. We have $\mathscr{E} \supset\left[\left(\operatorname{id}_{\mathscr{E}} \otimes \omega\right) \delta_{\mathscr{E}}(\xi) ; \xi \in \mathscr{E}, \omega \in \mathcal{B}(\mathscr{H})_{*}\right]$ (cf. 2.3.6). To obtain the converse inclusion, we combine the fact that there exists $\omega \in \mathcal{B}(\mathscr{H})_{*}$ such that $\left(\mathrm{id}_{\mathscr{E}} \otimes \omega\right)\left(q_{\beta_{\mathscr{E}} \alpha}\right)=1_{\mathscr{E}}$ with the formula $\left[\delta_{\mathscr{E}}(\mathscr{E})\left(1_{B} \otimes S\right)\right]=q_{\beta_{\mathscr{E}} \alpha}(\mathscr{E} \otimes S)$.

Now, we can state the main results of this chapter.

6.1.25 THEOREM. - Let $A$ be a $\mathcal{G}$ - $C^{*}$-algebra and $\mathscr{E}$ a Hilbert $A$-module acted upon by $\mathcal{G}$. Let $J:=\mathcal{K}(\mathscr{E} \oplus A)$ be the associated linking $C^{*}$-algebra endowed with the action $\left(\beta_{J}, \delta_{J}\right)$ defined in 6.1.11 If $\mathcal{G}$ is semi-regular (resp. regular), then the action $\left(\beta_{J}, \delta_{J}\right)$ is weakly (resp. strongly) continuous. 
Proof. Assume that $\mathcal{G}$ is semi-regular. Denote by $T:=\left[\left(\operatorname{id}_{J} \otimes \omega\right) \delta_{J}(x) ; x \in J, \omega \in \mathcal{B}(\mathscr{H})_{*}\right]$ the $\mathrm{C}^{*}$-algebra of continuous elements ( $\mathrm{cf} 5.3 .4$ ). By combining the compatibility of $\delta_{J}$ with $\delta_{A}$ (resp. $\delta_{\mathscr{E}}$ ) with the fact that $\left(\beta_{A}, \delta_{A}\right)$ is (weakly) continuous (resp. Lemma 6.1.24), we obtain $\iota_{A}(A) \subset T$ (resp. $\iota_{\mathscr{E}}(\mathscr{E}) \subset T$ ). Hence, $J \subset T$. The converse inclusion also holds since $\delta_{J}(J) \subset \widetilde{\mathcal{M}}(J \otimes S)$. Hence, $\left(\beta_{J}, \delta_{J}\right)$ is weakly continuous. It follows from 5.3.6 that the action $\left(\beta_{J}, \delta_{J}\right)$ is strongly continuous if $\mathcal{G}$ is regular.

6.1.26 CoRollary. - Let $\mathscr{E}$ be a Hilbert A-module. If the quantum groupoid $\mathcal{G}$ is regular, then any action of $\mathcal{G}$ on $\mathscr{E}$ is continuous.

Proof. This is an immediate consequence of 6.1.21 and 6.1.25.

\subsection{Case of a colinking measured quantum groupoid}

Let us fix a colinking measured quantum groupoid $\mathcal{G}:=\mathcal{G}_{\mathrm{G}_{1}, \mathrm{G}_{2}}$ between two monoidally equivalent locally compact quantum groups $\mathrm{G}_{1}$ and $\mathrm{G}_{2}$. Let $\left(A, \beta_{A}, \delta_{A}\right)$ be a $\mathcal{G}$-C $\mathrm{C}^{*}$-algebra. We follow all the notations of $\$ 3.3$ (resp. 5.2.1 and 5.2.2) concerning the objects associated with $\mathcal{G}$ (resp. $\left.\left(A, \beta_{A}, \delta_{A}\right)\right)$.

In the following, we provide a description of Hilbert modules acted upon by $\mathcal{G}$ in terms of Hilbert modules acted upon by $\mathrm{G}_{1}$ and $\mathbb{G}_{2}$. Let us fix a Hilbert $A$-module $\mathscr{E}$ endowed with an action $\left(\beta_{\mathscr{E}}, \delta_{\mathscr{E}}\right)$ of $\mathcal{G}$.

6.2.1 Notations. - We introduce some useful notations to describe the action $\left(\beta_{\mathscr{E}}, \delta_{\mathscr{E}}\right)$.

- Let $q_{\mathscr{E}, j}:=\beta_{\mathscr{E}}\left(\varepsilon_{j}\right)$ for $j=1,2$. Note that $q_{\mathscr{E}, 1}$ and $q_{\mathscr{E}, 2}$ are orthogonal self-adjoint projections of $\mathcal{L}(\mathscr{E})$ and $q_{\mathscr{E}, 1}+q_{\mathscr{E}, 2}=1_{\mathscr{E}}$.

- Let $J:=\mathcal{K}(\mathscr{E} \oplus A)$ be the linking $C^{*}$-algebra associated with $\mathscr{E}$ endowed with the action $\left(\beta_{I}, \delta_{I}\right)$ of $\mathcal{G}$ (cf. 6.1.11 b)). Since $\beta_{I}\left(\mathbb{C}^{2}\right) \subset \mathcal{Z}(\mathcal{M}(J))$ (cf. 3.2.3 [2]), we have $\beta_{\mathscr{E}}(n) \xi=\xi \beta_{A}(n)$ in $\mathcal{L}(A, \mathscr{E})$ for all $n \in \mathbb{C}^{2}$ and $\xi \in \mathscr{E}$, i.e. $\left(\beta_{\mathscr{E}}(n) \xi\right) a=\xi\left(\beta_{A}(n) a\right)$ for all $n \in \mathbb{C}^{2}, \xi \in \mathscr{E}$ and $a \in A$. Hence,

$$
\left(q_{\mathscr{E}, j} \xi\right) a=\xi\left(q_{A, j} a\right), \quad \text { for all } \xi \in \mathscr{E}, a \in A, j=1,2 .
$$

In particular, we have

$$
\left\langle q_{\mathscr{E}, j} \xi, q_{\mathscr{E}, j} \eta\right\rangle=q_{A, j}\langle\xi, \eta\rangle, \quad \text { for all } \xi, \eta \in \mathscr{E} .
$$

Indeed, fix $\xi, \eta \in \mathscr{E}$ and write $\xi=\xi^{\prime} a$ and $\eta=\eta^{\prime} b$ with $\xi^{\prime}, \eta^{\prime} \in \mathscr{E}$ and $a, b \in A$. Since the projection $q_{A, j}$ is central in $A$, we have $\left\langle q_{\mathscr{E}, j} \xi^{\prime}, q_{\mathscr{E}, j} \eta\right\rangle=\left\langle\left(q_{\mathscr{E}, j} \xi^{\prime}\right) a,\left(q_{\mathscr{E}, j} \eta^{\prime}\right) b\right\rangle=$ $\left\langle\xi^{\prime}\left(q_{A, j} a\right), \eta^{\prime}\left(q_{A, j} b\right)\right\rangle=q_{A, j} a^{*}\left\langle\xi^{\prime}, \eta^{\prime}\right\rangle b=q_{A, j}\left\langle\xi_{j}, \eta\right\rangle$. For $j=1,2$, we then define the following Hilbert $A_{j}$-module $\mathscr{E}_{j}:=q_{\mathscr{E}, j} \mathscr{E}^{\circ}$. Note that $\mathscr{E}=\mathscr{E}_{1} \oplus \mathscr{E}_{2}$.

- For $j, k=1,2$, let $\Pi_{j}^{k}: \mathscr{E}_{k} \otimes S_{k j} \rightarrow \mathscr{E} \otimes S$. be the inclusion map. It is clear that $\Pi_{j}^{k}$ is a $\pi_{j}^{k}$-compatible operator (cf. 8.3.1). We can consider its canonical linear extension $\Pi_{j}^{k}: \mathcal{L}\left(A_{k} \otimes S_{k j}, \mathscr{E}_{k} \otimes S_{k j}\right) \rightarrow \mathcal{L}(A \otimes S, \mathscr{E} \otimes S)$, up to the canonical injective maps $\mathscr{E}_{k} \otimes S_{k j} \rightarrow \mathcal{L}\left(A_{k} \otimes S_{k j}, \mathscr{E}_{k} \otimes S_{k j}\right)$ and $\mathscr{E} \otimes S \rightarrow \mathcal{L}(A \otimes S, \mathscr{E} \otimes S)$, defined by $\Pi_{j}^{k}(T)(x):=\Pi_{j}^{k} \circ T\left(\left(q_{A, k} \otimes p_{k j}\right) x\right)$ for all $T \in \mathcal{L}\left(A_{k} \otimes S_{k j}, \mathscr{E}_{k} \otimes S_{k j}\right)$ and $x \in A \otimes S$.

6.2.2 LEMMA. - With the above notations and hypotheses, we have a canonical unitary equivalence of Hilbert $A \otimes S$-modules $\mathscr{E} \otimes \delta_{A}(A \otimes S)=\bigoplus_{j, k=1,2} \mathscr{E}_{j} \otimes_{\delta_{A_{j}}^{k}}\left(A_{k} \otimes S_{k j}\right)$.

Proof. This is a straightforward verification to see that we define a unitary adjointable operator by the following formula:

$$
\mathscr{E} \otimes_{\delta_{A}}(A \otimes S) \rightarrow \bigoplus_{j, k=1,2} \mathscr{E}_{j} \otimes_{\delta_{A_{j}}^{k}}\left(A_{k} \otimes S_{k j}\right) ; \xi \otimes_{\delta_{A}} x \mapsto \bigoplus_{j, k=1,2} q_{\mathscr{E}, j} \xi \otimes_{\delta_{A_{j}}^{k}}\left(q_{A, k} \otimes p_{k j}\right) x .
$$


6.2.3 Proposition-Definition. - Let $\mathcal{V} \in \mathcal{L}\left(\mathscr{E} \otimes \delta_{A}(A \otimes S), \mathscr{E} \otimes S\right)$ be the isometry associated with the action $\left(\beta_{\mathscr{E}}, \delta_{\mathscr{E}}\right)$ (cf.6.1.5 a). For all $j, k=1,2$, there exists a unique unitary

such that

$$
\mathcal{V}_{j}^{k} \in \mathcal{L}\left(\mathscr{E}_{j} \otimes_{\delta_{A_{j}}^{k}}\left(A_{k} \otimes S_{k j}\right), \mathscr{E}_{k} \otimes S_{k j}\right)
$$

$$
\mathcal{V}\left(\xi \otimes_{\delta_{A}} x\right)=\sum_{j, k=1,2} \mathcal{V}_{j}^{k}\left(q_{\mathscr{E}, j} \xi \otimes_{\delta_{A_{j}}^{k}}\left(q_{A, k} \otimes p_{k j}\right) x\right), \quad \text { for all } \xi \in \mathscr{E} \text { and } x \in A \otimes S .
$$

Proof. Let $j, k=1,2$. Fix $\xi \in \mathscr{E}, x \in A \otimes S$ and write $x=x^{\prime} x^{\prime \prime}$ with $x^{\prime}, x^{\prime \prime} \in A \otimes S$. We have

$$
\begin{array}{rlr}
\mathcal{V}\left(q_{\mathscr{E}, j} \xi \otimes_{\delta_{A}}\left(q_{A, k} \otimes p_{k j}\right) x\right) & =\left(1_{\mathscr{E}} \otimes \beta\left(\varepsilon_{j}\right)\right) \mathcal{V}\left(\xi \otimes_{\delta_{A}}\left(q_{A, k} \otimes p_{k j}\right) x\right) \\
& =\left(1_{\mathscr{E}} \otimes \beta\left(\varepsilon_{j}\right)\right) \mathcal{V}\left(\xi \otimes_{\delta_{A}} x^{\prime}\left(q_{A, k} \otimes p_{k j}\right) x^{\prime \prime}\right) & \left(q_{A, k}, p_{k j} \text { are central }\right) . \\
& =\left(1_{\mathscr{E}} \otimes \beta\left(\varepsilon_{j}\right)\right) \mathcal{V}\left(\xi \otimes_{\delta_{A}} x^{\prime}\right)\left(q_{A, k} \otimes p_{k j}\right) x^{\prime \prime} & (\mathcal{V} \text { is } A \otimes S \text {-linear }) .
\end{array}
$$

Now if $\eta \in \mathscr{E}, y, s \in S$ and $a \in A$, we have

$$
\begin{aligned}
\left(1_{\mathscr{E}} \otimes \beta\left(\varepsilon_{j}\right)\right)(\eta \otimes y)\left(q_{A, k} \otimes p_{k j}\right)(a \otimes s) & =\eta\left(q_{A, k} a\right) \otimes \beta\left(\varepsilon_{j}\right) y p_{k j} s \\
& =\eta\left(q_{A, k} a\right) \otimes p_{k j} y s \\
& =q_{\mathscr{E}, k} \eta a \otimes p_{k j} y s \in \mathscr{E}_{k} \otimes S_{k j}
\end{aligned}
$$

by using 6.10 and the fact that $p_{k j}$ is central in $S$. In particular, for all $\xi \in \mathscr{E}$ and $x \in A \otimes S$ we have $\mathcal{V}\left(q_{\mathscr{E}, j} \xi \otimes_{\delta_{A}}\left(q_{A, k} \otimes p_{k j}\right) x\right) \in \mathscr{E}_{k} \otimes S_{k j}$. By combining the fact that $\mathcal{V}$ is isometric with the fact that $x \delta_{A_{j}}^{k}(a)=x \delta_{A}(a)$ for all $a \in A_{j}$ and $x \in A_{k} \otimes S_{k j}$, we obtain a well-defined isometric $A_{k} \otimes S_{k j}$-linear map

$$
\mathcal{V}_{j}^{k}: \mathscr{E}_{j} \otimes_{\delta_{A_{j}}^{k}}\left(A_{k} \otimes S_{k j}\right) \rightarrow \mathscr{E}_{k} \otimes S_{k j} ; \xi \otimes_{\delta_{A_{j}}^{k}} x \mapsto \mathcal{V}\left(\xi \otimes_{\delta_{A}} x\right)
$$

It follows from $\left.\operatorname{im}(\mathcal{V})=q_{\beta_{\mathscr{E}} \alpha}(\mathscr{E} \otimes S) \sqrt{6.1 .4} 1\right)$ that $\mathcal{V}_{j}^{k}$ is surjective. As a result, we have $\mathcal{V}_{j}^{k} \in \mathcal{L}\left(\mathscr{E}_{j} \otimes_{\delta_{A_{j}}^{k}}\left(A_{k} \otimes S_{k j}\right), \mathscr{E}_{k} \otimes S_{k j}\right)$ and $\mathcal{V}_{j}^{k}$ is unitary.

For $j, k, l=1,2$ we have the following set of unitary equivalences of Hilbert modules:

$$
\begin{aligned}
& A_{j} \otimes_{\delta_{A_{j}}^{k}}\left(A_{k} \otimes S_{k j}\right) \rightarrow A_{k} \otimes S_{k j} \\
& a \otimes_{\delta_{A_{j}}^{k}} x \mapsto \delta_{A_{j}}^{k}(a) x \\
& \left(A_{k} \otimes S_{k j}\right) \otimes_{\delta_{A_{k}}^{l} \otimes \operatorname{id}_{S_{k j}}}\left(A_{l} \otimes S_{l k} \otimes S_{k j}\right) \rightarrow A_{l} \otimes S_{l k} \otimes S_{k j}
\end{aligned}
$$

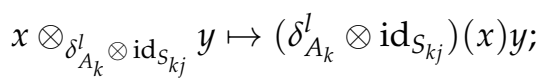

$$
\begin{aligned}
& \left(A_{l} \otimes S_{l j}\right) \otimes_{\mathrm{id}_{A_{l}} \otimes \delta_{l j}^{k}}\left(A_{l} \otimes S_{l k} \otimes S_{k j}\right) \rightarrow A_{l} \otimes S_{l k} \otimes S_{k j} \\
& x \otimes_{\mathrm{id}_{A_{l}} \otimes \delta_{l j}^{k}} y \mapsto\left(\mathrm{id}_{A_{l}} \otimes \delta_{l j}^{k}\right)(x) y ; \\
& \left(\mathscr{E}_{j} \otimes_{\delta_{A_{j}}^{k}}\left(A_{k} \otimes S_{k j}\right)\right) \otimes_{\delta_{A_{k}}^{l} \otimes \operatorname{id}_{S_{k j}}}\left(A_{l} \otimes S_{l k} \otimes S_{k j}\right) \rightarrow \mathscr{E}_{j} \otimes_{\left(\delta_{A_{k}}^{l} \otimes \operatorname{id}_{S_{k j}}\right) \delta_{A_{j}}^{k}}\left(A_{l} \otimes S_{l k} \otimes S_{k j}\right)
\end{aligned}
$$

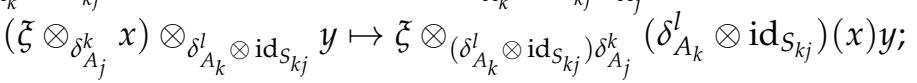

$$
\begin{aligned}
& \left(\mathscr{E}_{j} \otimes_{\delta_{A_{j}}^{l}}\left(A_{l} \otimes S_{l j}\right)\right) \otimes_{\mathrm{id}_{A_{l}} \otimes \delta_{l j}^{k}}\left(A_{l} \otimes S_{l k} \otimes S_{k j}\right) \rightarrow \mathscr{E}_{j} \otimes_{\left(\mathrm{id}_{A_{l}} \otimes \delta_{l j}^{k}\right) \delta_{A_{j}}^{l}}\left(A_{l} \otimes S_{l k} \otimes S_{k j}\right) \\
& \left(\xi \otimes_{\delta_{A_{j}}^{l}} x\right) \otimes_{\mathrm{id}_{A_{l}} \otimes \delta_{l j}^{k}} y \mapsto \xi \otimes_{\left(\mathrm{id}_{A_{l}} \otimes \delta_{l j}^{k}\right) \delta_{A_{j}}^{l}}\left(\mathrm{id}_{A_{l}} \otimes \delta_{l j}^{k}\right)(x) y ;
\end{aligned}
$$

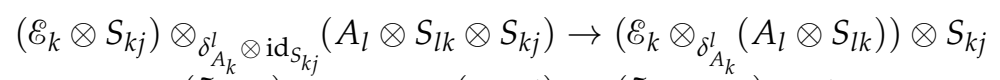

$$
\begin{aligned}
& (\xi \otimes s) \otimes_{\delta_{A_{k}}^{l}} \otimes \operatorname{id}_{s_{k j}}(x \otimes t) \mapsto\left(\xi \otimes_{\delta_{A_{k}}^{l}} x\right) \otimes s t ; \\
& \left(\mathscr{E}_{l} \otimes S_{l j}\right) \otimes_{\mathrm{id}_{A_{l}} \otimes \delta_{l j}^{k}}\left(A_{l} \otimes S_{l k} \otimes S_{k j}\right) \rightarrow \mathscr{E}_{l} \otimes S_{l k} \otimes S_{k j} \\
& \xi \otimes_{\mathrm{id}_{A_{l}} \otimes \delta_{l j}^{k}} y \mapsto\left(\mathrm{id}_{\mathscr{E}_{l}} \otimes \delta_{l j}^{k}\right)(\xi) y .
\end{aligned}
$$


6.2.4 Proposition. - For all $j, k, l=1,2$, we have

$$
\left(\mathcal{V}_{k}^{l} \otimes_{\mathrm{C}} \mathrm{id}_{S_{k j}}\right)\left(\mathcal{V}_{j}^{k} \otimes_{\delta_{A_{k}}^{l} \otimes \mathrm{id}_{S_{k j}}} 1\right)=\mathcal{V}_{j}^{l} \otimes_{\mathrm{id}_{A_{l}} \otimes \delta_{l j}^{k}} 1 .
$$

For $j, k, l=1,2, \mathcal{V}_{k}^{l} \otimes_{\mathbb{C}} \mathrm{id}_{S_{k j}} \in \mathcal{L}\left(\left(\mathscr{E}_{k} \otimes S_{k j}\right) \otimes_{\delta_{A_{k}}^{l}} \otimes \mathrm{id}_{S_{k j}}\left(A_{l} \otimes S_{l k} \otimes S_{k j}\right), \mathscr{E}_{l} \otimes S_{l k} \otimes S_{k j}\right)$

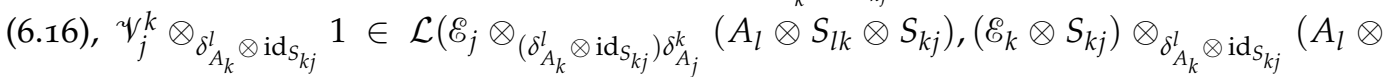
$\left.\left.S_{l k} \otimes S_{k j}\right)\right) \sqrt{6.14}$ and $\mathcal{V}_{j}^{l} \otimes_{\mathrm{id}_{A_{l}} \otimes \delta_{l j}^{k}} 1 \in \mathcal{L}\left(\mathscr{E}_{j} \otimes_{\left(\mathrm{id}_{A_{l}} \otimes \delta_{l j}^{k}\right) \delta_{A_{j}}^{l}}\left(A_{l} \otimes S_{l k} \otimes S_{k j}\right), \mathscr{E}_{l} \otimes S_{l k} \otimes S_{k j}\right)$ 6.17). Moreover, the composition $\left(\mathcal{V}_{k}^{l} \otimes_{\mathrm{C}} \mathrm{id}_{S_{k j}}\right)\left(\mathcal{V}_{j}^{k} \otimes_{\delta_{A_{k}}^{l}} \otimes \mathrm{id}_{S_{k j}} 1\right)$ does make sense since $\left(\delta_{A_{k}}^{l} \otimes \mathrm{id}_{S_{k j}}\right) \delta_{A_{j}}^{k}=\left(\mathrm{id}_{A_{l}} \otimes \delta_{l j}^{k}\right) \delta_{A_{j}}^{l}$

Proof. Straightforward consequence of $\left(\mathcal{V} \otimes_{\mathbb{C}} \mathrm{id}_{S}\right)\left(\mathcal{V} \otimes_{\delta_{A} \otimes_{i d}} 1\right)=\mathscr{V} \otimes_{\mathrm{id}_{A} \otimes \delta} 1$.

6.2.5 Proposition-Definition. - For $j, k=1,2$, let $\delta_{\mathscr{E}_{j}}^{k}: \mathscr{E}_{j} \rightarrow \mathcal{L}\left(A_{k} \otimes S_{k j}, \mathscr{E}_{k} \otimes S_{k j}\right)$ be the linear map defined by

$$
\delta_{\mathscr{E}_{j}}^{k}(\xi) x:=\mathcal{V}_{j}^{k}\left(\xi \otimes_{\delta_{A_{j}}^{k}} x\right), \quad \text { for all } \xi \in \mathscr{E}_{j} \text { and } x \in A_{k} \otimes S_{k j}
$$

For all $j, k, l=1,2$, we have:

(i) $\delta_{\mathscr{E}}(\xi)=\sum_{k, j=1,2} \Pi_{j}^{k} \circ \delta_{\mathscr{E}_{j}}^{k}\left(q_{\mathscr{E}, j} \xi\right)$, for all $\xi \in \mathscr{E}$;

(ii) $\delta_{\mathscr{E}_{j}}^{k}\left(\mathscr{E}_{j}\right) \subset \widetilde{\mathcal{M}}\left(\mathscr{E}_{k} \otimes S_{k j}\right)$;

(iii) $\delta_{\mathscr{E}_{j}}^{k}(\xi a)=\delta_{\mathscr{E}_{j}}^{k}(\xi) \delta_{A_{j}}^{k}(a)$ and $\left\langle\delta_{\mathscr{E}_{j}}^{k}(\xi), \delta_{\mathscr{E}_{j}}^{k}(\eta)\right\rangle=\delta_{A_{j}}^{k}(\langle\xi, \eta\rangle)$, for all $\xi, \eta \in \mathscr{E}_{j}$ and $a \in A_{j}$;

(iv) $\left[\delta_{\mathscr{E}_{j}}^{k}\left(\mathscr{E}_{j}\right)\left(1_{A_{k}} \otimes S_{k j}\right)\right]=\mathscr{E}_{k} \otimes S_{k j}$; in particular, we have

$$
\mathscr{E}_{k}=\left[\left(\operatorname{id}_{\mathscr{E}_{k}} \otimes \omega\right) \delta_{\mathscr{E}_{j}}^{k}(\xi) ; \omega \in \mathcal{B}\left(\mathscr{H}_{k j}\right)_{*}, \xi \in \mathscr{E}_{j}\right] \quad(c f \cdot 2.3 .6 .
$$

(v) $\delta_{\mathscr{E}_{k}}^{l} \otimes \mathrm{id}_{S_{k j}}\left(\right.$ resp. $\left.\mathrm{id}_{\mathscr{E}_{l}} \otimes \delta_{l j}^{k}\right)$ extends to a linear map from $\mathcal{L}\left(A_{k} \otimes S_{k j}, \mathscr{E}_{k} \otimes S_{k j}\right)$ (resp. $\left.\mathcal{L}\left(A_{l} \otimes S_{l j}, \mathscr{E}_{l} \otimes S_{l j}\right)\right)$ to $\mathcal{L}\left(A_{l} \otimes S_{l k} \otimes S_{k j}, \mathscr{E}_{l} \otimes S_{l k} \otimes S_{k j}\right)$ and for all $\xi \in \mathscr{E}_{j}$ we have

$$
\left(\delta_{\mathscr{E}_{k}}^{l} \otimes \mathrm{id}_{S_{k j}}\right) \delta_{\mathscr{E}_{j}}^{k}(\xi)=\left(\mathrm{id}_{\mathscr{E}_{l}} \otimes \delta_{l j}^{k}\right) \delta_{\mathscr{E}_{j}}^{l}(\xi) \in \mathcal{L}\left(A_{l} \otimes S_{l k} \otimes S_{k j}, \mathscr{E}_{l} \otimes S_{l k} \otimes S_{k j}\right)
$$

(vi) if $\mathscr{E}$ is a $\mathcal{G}$-equivariant Hilbert $A$-module, then we have $\left[\left(1_{\mathscr{E}_{k}} \otimes S_{k j}\right) \delta_{\mathscr{E}_{j}}^{k}\left(\mathscr{E}_{j}\right)\right]=\mathscr{E}_{k} \otimes S_{k j}$.

If $\mathscr{E}$ is a $\mathcal{G}$-equivariant Hilbert module, then $\left(\mathscr{E}_{j}, \delta_{\mathscr{E}_{j}}^{j}\right)$ is a $\mathbb{G}_{j}$-equivariant Hilbert $A_{j}$-module and $\mathcal{V}_{j}^{j}$ is the associated unitary.

Proof. It is clear that $\delta_{\mathscr{E}_{j}}^{k}: \mathscr{E}_{j} \rightarrow \mathcal{L}\left(A_{k} \otimes S_{k j}, \mathscr{E}_{k} \otimes S_{k j}\right)$ is a well-defined linear map. Moreover, statement (i) follows straightforwardly from 6.2.3 and the fact that $\delta_{\mathscr{E}}(\xi) x=\mathcal{V}\left(\xi \otimes_{\delta_{A}} x\right)$ for all $\xi \in \mathscr{E}$ and $x \in A \otimes S$. Let $\xi \in \mathscr{E}_{j}$ and $s \in S_{k j}$. We have

$$
\Pi_{j}^{k}\left(\delta_{\mathscr{E}_{j}}^{k}(\xi)\left(1_{A_{k}} \otimes s\right)\right)=\delta_{\mathscr{E}}(\xi)\left(1_{A} \otimes s\right) \quad \text { and } \quad \Pi_{j}^{k}\left(\left(1_{\mathscr{E}_{k}} \otimes s\right) \delta_{\mathscr{E}_{j}}^{k}(\xi)\right)=\left(1_{\mathscr{E}} \otimes s\right) \delta_{\mathscr{E}}(\xi)
$$

It then follows from $\delta_{\mathscr{E}}(\mathscr{E}) \subset \widetilde{\mathcal{M}}(\mathscr{E} \otimes S)$ that $\Pi_{j}^{k}\left(\delta_{\mathscr{E}_{j}}^{k}(\xi)\left(1_{A_{k}} \otimes s\right)\right)$ and $\Pi_{j}^{k}\left(\left(1_{\mathscr{E}_{k}} \otimes s\right) \delta_{\mathscr{E}_{j}}^{k}(\xi)\right)$ belong to $\mathscr{E} \otimes S$. Moreover, $\left(q_{\mathscr{E}, k} \otimes p_{k j}\right) \Pi_{j}^{k}(T)=\Pi_{j}^{k}(T)$ for all $T \in \mathcal{L}\left(A_{k} \otimes S_{k j}, \mathscr{E}_{k} \otimes S_{k j}\right)$; hence, $\Pi_{j}^{k}\left(\delta_{\mathscr{E}_{j}}^{k}(\xi)\left(1_{A_{k}} \otimes s\right)\right) \in \Pi_{j}^{k}\left(\mathscr{E}_{k} \otimes S_{k j}\right)$ and $\Pi_{j}^{k}\left(\left(1_{\mathscr{E}_{k}} \otimes s\right) \delta_{\mathscr{E}_{j}}^{k}(\xi)\right) \in \Pi_{j}^{k}\left(\mathscr{E}_{k} \otimes S_{k j}\right)$. It then follows that $\delta_{\mathscr{E}_{j}}^{k}(\xi)\left(1_{A_{k}} \otimes s\right)$ and $\left(1_{\mathscr{E}_{k}} \otimes s\right) \delta_{\mathscr{E}_{j}}^{k}(\xi)$ belong to $\mathscr{E}_{k} \otimes S_{k j}$ by injectivity of 
$\Pi_{j}^{k}: \mathcal{L}\left(A_{k} \otimes S_{k j}, \mathscr{E}_{k} \otimes S_{k j}\right) \rightarrow \mathcal{L}(A \otimes S, \mathscr{E} \otimes S)$. Hence, statement (ii) is proved.

Let $\xi, \eta \in \mathscr{E}_{j}$. We have

$$
\begin{aligned}
\pi_{j}^{k}\left(\left\langle\delta_{\mathscr{E}_{j}}^{k}(\xi), \delta_{\mathscr{E}_{j}}^{k}(\eta)\right\rangle\right) & =\left\langle\Pi_{j}^{k}\left(\delta_{\mathscr{E}_{j}}^{k}(\xi)\right), \Pi_{j}^{k}\left(\delta_{\mathscr{E}_{j}}^{k}(\eta)\right)\right\rangle \\
& =\left\langle\left(q_{\mathscr{E}, k} \otimes p_{k j}\right) \delta_{\mathscr{E}}(\xi),\left(q_{\mathscr{E}, k} \otimes p_{k j}\right) \delta_{\mathscr{E}}(\eta)\right\rangle \\
& =\left(q_{A, k} \otimes p_{k j}\right) \delta_{A}(\langle\xi, \eta\rangle) \\
& =\pi_{j}^{k}\left(\delta_{A_{j}}^{k}(\langle\xi, \eta\rangle)\right) .
\end{aligned}
$$

Hence $\left\langle\delta_{\mathscr{E}_{j}}^{k}(\xi), \delta_{\mathscr{E}_{j}}^{k}(\eta)\right\rangle=\delta_{A_{j}}^{k}(\langle\xi, \eta\rangle)$ by injectivity of $\pi_{j}^{k}$. The first formula of statement (iii) is derived immediately from the definition of $\delta_{\mathscr{E}_{j}}^{k}$.

The surjectivity of $\mathcal{V}_{j}^{k}$ is just a restatement of $\left[\delta_{\mathscr{E}_{j}}^{k}\left(\mathscr{E}_{j}\right)\left(A_{k} \otimes S_{k j}\right)\right]=\mathscr{E}_{k} \otimes S_{k j}$. The identity $\left[\delta_{\mathscr{E}_{j}}^{k}\left(\mathscr{E}_{j}\right)\left(1_{A_{k}} \otimes S_{k j}\right)\right]=\mathscr{E}_{k} \otimes S_{k j}$ follows by combining the previous formula with the first relation of (iii) and the relation $\left[\delta_{A_{j}}^{k}\left(A_{j}\right)\left(1_{A_{k}} \otimes S_{k j}\right)\right]=A_{k} \otimes S_{k j}$. Let us prove the formula $\mathscr{E}_{k}=\left[\left(\operatorname{id}_{\mathscr{E}_{k}} \otimes \omega\right) \delta_{\mathscr{E}_{j}}^{k}(\xi) ; \omega \in \mathcal{B}\left(\mathscr{H}_{k j}\right)_{*}, \xi \in \mathscr{E}_{j}\right]$. By statement (ii) and 2.3.6, we already have the relation $\mathscr{E}_{k} \supset\left[\left(\operatorname{id}_{\mathscr{E}_{k}} \otimes \omega\right) \delta_{\mathscr{E}_{j}}^{k}(\xi) ; \omega \in \mathcal{B}\left(\mathscr{H}_{k j}\right)_{*}, \xi \in \mathscr{E}_{j}\right]$. Conversely, let us fix $\eta \in \mathscr{E}_{k}$. Let $\omega \in \mathcal{B}\left(\mathscr{H}_{k j}\right)_{*}$ and $s \in S_{k j}$ such that $\omega(s)=1$. It then follows from the formula $\left[\delta_{\mathscr{E}_{j}}^{k}\left(\mathscr{E}_{j}\right)\left(1_{A_{k}} \otimes S_{k j}\right)\right]=\mathscr{E}_{k} \otimes S_{k j}$ that $\eta=\left(\operatorname{id}_{\mathscr{E}_{k}} \otimes \omega\right)(\eta \otimes s)$ is the norm limit of finite sums of elements of the form $\left(\operatorname{id}_{\mathscr{E}_{k}} \otimes \omega\right)\left(\delta_{\mathscr{E}_{j}}^{k}(\xi)\left(1_{A_{k}} \otimes y\right)\right)=\left(\operatorname{id}_{\mathscr{E}_{k}} \otimes y \omega\right) \delta_{\mathscr{E}_{j}}^{k}(\xi)$, where $\xi \in \mathscr{E}_{j}$ and $y \in S$. Therefore, statement (iv) is proved.

By using the identifications (6.12) and (6.16) (resp. 6.13) and (6.17)), the linear map

$$
\begin{array}{r}
\delta_{\mathscr{E}_{k}}^{l} \otimes \mathrm{id}_{S_{k j}}: \mathcal{L}\left(A_{k} \otimes S_{k j}, \mathscr{E}_{k} \otimes S_{k j}\right) \rightarrow \mathcal{L}\left(A_{l} \otimes S_{l k} \otimes S_{k j}, \mathscr{E}_{l} \otimes S_{l k} \otimes S_{k j}\right) \\
\left(\text { resp. } \mathrm{id}_{\mathscr{E}_{l}} \otimes \delta_{l j}^{k}: \mathcal{L}\left(A_{l} \otimes S_{l j}, \mathscr{E}_{l} \otimes S_{l j}\right) \rightarrow \mathcal{L}\left(A_{l} \otimes S_{l k} \otimes S_{k j}, \mathscr{E}_{l} \otimes S_{l k} \otimes S_{k j}\right)\right)
\end{array}
$$

is defined for all $T \in \mathcal{L}\left(A_{k} \otimes S_{k j}, \mathscr{E}_{k} \otimes S_{k j}\right)$ (resp. $\left.T \in \mathcal{L}\left(A_{l} \otimes S_{l j}, \mathscr{E}_{l} \otimes S_{l j}\right)\right)$ by

$$
\left(\delta_{\mathscr{E}_{k}}^{l} \otimes \operatorname{id}_{S_{k j}}\right)(T):=\left(\mathcal{Y}_{k}^{j} \otimes 1_{S_{k j}}\right)\left(T \otimes_{\delta_{A_{k}}^{l} \otimes \operatorname{id}_{S_{k j}}} 1\right) \quad\left(\text { resp. }\left(\operatorname{id}_{\mathscr{E}_{l}} \otimes \delta_{l j}^{k}\right)(T):=T \otimes_{\operatorname{id}_{A_{l}} \otimes \delta_{l j}^{k}} 1\right) .
$$

The relation $\left(\delta_{\mathscr{E}_{k}}^{l} \otimes \operatorname{id}_{S_{k j}}\right) \delta_{\mathscr{E}_{j}}^{k}(\xi)=\left(\mathrm{id}_{\mathscr{E}_{l}} \otimes \delta_{l j}^{k}\right) \delta_{\mathscr{E}_{j}}^{l}(\xi)$ for $\xi \in \mathscr{E}_{j}$ is then derived from 6.2.4 as in the proof of 6.1 .5 Assume that $\left(\beta_{\mathscr{E}}, \delta_{\mathscr{E}}\right)$ is continuous. Since $p_{k j}$ is central in $S$, we have

$$
\begin{aligned}
\Pi_{j}^{k}\left(\mathscr{E}_{k} \otimes S_{k j}\right) & =\left(q_{\mathscr{E}, j} \otimes p_{k j}\right)(\mathscr{E} \otimes S) q_{\beta_{A} \alpha} \\
& =\left(q_{\mathscr{E}, j} \otimes p_{k j}\right)\left[\left(1_{\mathscr{E}} \otimes S\right) \delta_{\mathscr{E}}(\mathscr{E})\right] \\
& =\Pi_{j}^{k}\left[\left(1_{\mathscr{E}_{k}} \otimes S_{k j}\right) \delta_{\mathscr{E}_{j}}^{k}\left(\mathscr{E}_{j}\right)\right]
\end{aligned}
$$

and statement (vi) is proved.

From this concrete description of $\mathcal{G}$-equivariant Hilbert $C^{*}$-modules, we can also provide a corresponding description of the $\mathcal{G}$-equivariant unitary equivalences between them.

6.2.6 Lemma. - Let $A$ and $B$ be $\mathcal{G}$-C*-algebras. Let $\mathscr{E}$ and $\mathscr{F}$ be Hilbert $C^{*}$-modules over $A$ and $B$ respectively acted upon by $\mathcal{G}$.

1. Let $\Phi: \mathscr{E} \rightarrow \mathscr{F}$ be a $\mathcal{G}$-equivariant unitary equivalence over a $\mathcal{G}$-equivariant ${ }^{*}$-isomorphism $\phi: A \rightarrow B$. For $j=1,2$, there exists a unique map $\Phi_{j}: \mathscr{E}_{j} \rightarrow \mathscr{F}_{j}$ satisfying the formula $\Phi(\xi)=\Phi_{1}\left(q_{\mathscr{E}, 1} \xi\right)+\Phi_{2}\left(q_{\mathscr{E}, 2} \xi\right)$ for all $\xi \in \mathscr{E}$. Moreover, we have:

(i) for $j=1,2$, the map $\Phi_{j}$ is a unitary equivalence over the *isomorphism $\phi_{j}: A_{j} \rightarrow B_{j}$ (cf. 5.2.3 1 );

(ii) for all $j, k=1,2$, we have

$$
\left(\Phi_{k} \otimes \operatorname{id}_{S_{k j}}\right) \circ \delta_{\mathscr{E}_{j}}^{k}=\delta_{\mathscr{F}_{j}}^{k} \circ \Phi_{j} .
$$


In particular, $\Phi_{j}$ is a $\mathbb{G}_{j}$-equivariant $\phi_{j}$-compatible unitary operator.

2. Conversely, for $j=1,2$ let $\Phi_{j}: \mathscr{E}_{j} \rightarrow \mathscr{F}_{j}$ be a $\mathbb{G}_{j}$-equivariant unitary equivalence over a $\mathrm{G}_{j}$-equivariant ${ }^{*}$-isomorphism $\phi_{j}: A_{j} \rightarrow B_{j}$ such that (5.1) and 6.18 . hold for all $j, k=1,2$. Then, the map $\Phi: \mathscr{E} \rightarrow \mathscr{F}$, defined by $\Phi(\xi):=\Phi_{1}\left(q_{\mathscr{E}, 1} \xi\right)+\Phi_{2}\left(q_{\mathscr{E}}, 2 \xi\right)$ for all $\xi \in \mathscr{E}$, is a $\mathcal{G}$-equivariant unitary equivalence over the $\mathcal{G}$-equivariant ${ }^{*}$-isomorphism $\phi: A \rightarrow B$ (cf. $5 \cdot 2 \cdot 32)$.

Proof. 1. Let $j=1,2$. Since $\Phi$ is $\mathcal{G}$-equivariant, we have $\Phi \circ q_{\mathscr{E}, j}=q_{\mathscr{F}, j} \circ \Phi$. It then follows that $\Phi\left(\mathscr{E}_{j}\right) \subset \mathscr{F}_{j}$. Let us denote $\Phi_{j}:=\left.\Phi\right|_{\mathscr{E}_{j}}: \mathscr{E}_{j} \rightarrow \mathscr{F}_{j}$. For $\xi \in \mathscr{E}$, we have $\xi=q \mathscr{E}, 1 \xi+q \mathscr{E}, 2 \xi$; hence, $\Phi(\xi)=\Phi_{1}\left(q_{\mathscr{E}, 1} \xi\right)+\Phi_{2}\left(q_{\mathscr{E}, 2} \xi\right)$. Moreover, such a decomposition of $\Phi$ is unique

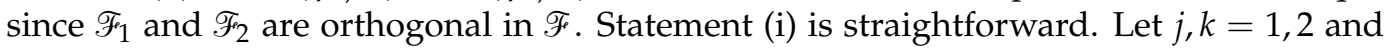
$x \in A_{k} \otimes S_{k j}$. For all $T \in \mathcal{L}\left(A_{k} \otimes S_{k j}, \mathscr{E}_{k} \otimes S_{k j}\right)$ we have

$$
\left(\Phi \otimes \mathrm{id}_{S}\right)\left(\Pi_{j}^{k}(T)\right)\left(\phi \otimes \mathrm{id}_{S}\right)(x)=\left(\Phi_{k} \otimes \mathrm{id}_{S_{k j}}\right)(T)\left(\phi_{k} \otimes \mathrm{id}_{S_{k j}}\right)(x) .
$$

In particular, $\left(\Phi \otimes \mathrm{id}_{S}\right)\left(\Pi_{j}^{k}\left(\delta_{\mathscr{E}_{j}}^{k}(\xi)\right)\right)\left(\phi \otimes \mathrm{id}_{S}\right)(x)=\left(\Phi_{k} \otimes \mathrm{id}_{S_{k j}}\right)\left(\delta_{\mathscr{E}_{j}}^{k}(\xi)\right)\left(\phi_{k} \otimes \mathrm{id}_{S_{k j}}\right)(x)$ for all $\xi \in \mathscr{E}_{j} ;$ hence, $\left(\Phi \otimes \mathrm{id}_{S}\right)\left(\delta_{\mathscr{E}}(\xi)\right)\left(\phi \otimes \mathrm{id}_{S}\right)(x)=\left(\Phi_{k} \otimes \mathrm{id}_{S_{k j}}\right)\left(\delta_{\mathscr{E}_{j}}^{k}(\xi)\right)\left(\phi_{k} \otimes \mathrm{id}_{S_{k j}}\right)(x) \sqrt[6.2 .5]{ }$ (i)) for all $\xi \in \mathscr{E}_{j}$. We also have $\delta_{\mathscr{F}}(\Phi(\xi))\left(q_{A, k} \otimes p_{k j}\right)=\Pi_{j}^{k}\left(\delta_{\mathscr{F}_{j}}^{k}\left(\Phi_{j}(\xi)\right)\right)$ for all $\xi \in \mathscr{E}_{j}$. Hence, $\delta_{\mathscr{F}}(\Phi(\xi))\left(\phi \otimes \mathrm{id}_{S}\right)(x)=\delta_{\mathscr{F}_{j}}^{k}\left(\Phi_{j}(\xi)\right)\left(\phi_{k} \otimes \mathrm{id}_{S_{k j}}\right)(x)$ for all $\xi \in \mathscr{E}_{j}$ and statement (ii) is proved.

2. Straightforward.

6.2.7 ExAmple. - Let $\left(\beta_{N}, \delta_{N}\right)$ be the trivial action (cf. 5.1.4). Let $i=1,2$. Consider the Hilbert $N$-module $\mathscr{E}:=\mathscr{H}_{i 1} \oplus \mathscr{H}_{i 2}$. Let $\mathcal{V} \in \mathcal{L}\left(\mathscr{E} \otimes_{\delta_{N}}(N \otimes S), \mathscr{E} \otimes S\right)$ and $\beta_{\mathscr{E}}: N \rightarrow \mathcal{L}(\mathscr{E})$ be the maps defined by the formulas:

$$
\mathcal{V}(\xi \otimes 1)=\sum_{k=1,2} V_{k j}^{i}(\xi \otimes 1), \quad \xi \in \mathscr{H}_{i j} \quad ; \quad \beta_{\mathscr{E}}\left(\varepsilon_{j}\right)=p_{i j}, \quad j=1,2 .
$$

Then, the pair $\left(\mathcal{V}, \beta_{\mathscr{E}}\right)$ is an action of $\mathcal{G}$ on $\mathscr{E}$.

\subsection{Induction of equivariant Hilbert $C^{*}$-modules}

Let $G_{1}$ and $G_{2}$ be two monoidally equivalent regular locally compact quantum groups.

Fix a $\mathbb{G}_{1}-C^{*}$-algebra $\left(A_{1}, \delta_{A_{1}}\right)$ and a $\mathbb{G}_{1}$-equivariant Hilbert $A_{1}$-module $\left(\mathscr{E}_{1}, \delta_{\mathscr{E}_{1}}\right)$. We denote by $J_{1}:=\mathcal{K}\left(\mathscr{E}_{1} \oplus A_{1}\right)$ the associated linking $C^{*}$-algebra endowed with the continuous action $\delta_{J_{1}}$ of $\mathrm{G}_{1}$.

6.3.1 Notations. - Let us fix some notations.

- Let $\mathrm{id}_{\mathscr{E}_{1}} \otimes \delta_{11}^{2}: \mathcal{L}\left(A_{1} \otimes S_{11}, \mathscr{E}_{1} \otimes S_{11}\right) \rightarrow \mathcal{L}\left(A_{1} \otimes S_{12} \otimes S_{21}, \mathscr{E}_{1} \otimes S_{12} \otimes S_{21}\right)$ be the unique linear extension of $\mathrm{id}_{\mathscr{E}_{1}} \otimes \delta_{11}^{2}: \mathscr{E}_{1} \otimes S_{11} \rightarrow \mathcal{L}\left(A_{1} \otimes S_{12} \otimes S_{21}, \mathscr{E}_{1} \otimes S_{12} \otimes S_{21}\right)$ such that $\left(\mathrm{id}_{\mathscr{E}_{1}} \otimes \delta_{11}^{2}\right)(T)\left(\mathrm{id}_{A_{1}} \otimes \delta_{11}^{2}\right)(x)=\left(\mathrm{id}_{\mathscr{E}_{1}} \otimes \delta_{11}^{2}\right)(T x)$ for all $x \in \mathcal{M}\left(A_{1} \otimes S_{11}\right)$ and $T \in \mathcal{L}\left(A_{1} \otimes S_{11}, \mathscr{E}_{1} \otimes S_{11}\right)$.

- Let $\delta_{\mathscr{E}_{1}}^{(2)}: \mathscr{E}_{1} \rightarrow \mathcal{L}\left(A_{1} \otimes S_{12} \otimes S_{21}, \mathscr{E}_{1} \otimes S_{12} \otimes S_{21}\right)$ be the linear map defined by $\delta_{\mathscr{E}_{1}}^{(2)}(\xi):=\left(\mathrm{id}_{\mathscr{E}_{1}} \otimes \delta_{11}^{2}\right) \delta_{\mathscr{E}_{1}}(\xi)$ for all $\xi \in \mathscr{E}_{1}$.

- Consider the Banach subspace of $\mathcal{L}\left(A_{1} \otimes S_{12}, \mathscr{E}_{1} \otimes S_{12}\right)$ defined by (cf. 2.3.6):

$$
\operatorname{Ind}_{\mathbb{G}_{1}}^{\mathbb{G}_{2}}\left(\mathscr{E}_{1}\right):=\left[\left(\operatorname{id}_{\mathscr{E}_{1} \otimes S_{12}} \otimes \omega\right) \delta_{\mathscr{E}_{1}}^{(2)}(\xi) ; \xi \in \mathscr{E}_{1}, \omega \in \mathcal{B}\left(\mathscr{H}_{21}\right)_{*}\right] .
$$

6.3.2 Proposition. - We have $\left[\operatorname{Ind}_{\mathbb{G}_{1}}^{\mathbb{G}_{2}}\left(\mathscr{E}_{1}\right)\left(1_{A_{1}} \otimes S_{12}\right)\right]=\mathscr{E}_{1} \otimes S_{12}=\left[\left(1_{\mathscr{E}_{1}} \otimes S_{11}\right) \operatorname{Ind}_{\mathrm{G}_{1}}^{\mathrm{G}_{2}}\left(\mathscr{E}_{1}\right)\right]$. In particular, $\operatorname{Ind}_{\mathrm{G}_{1}}^{\mathrm{G}_{2}}\left(\mathscr{E}_{1}\right) \subset \widetilde{\mathcal{M}}\left(\mathscr{E}_{1} \otimes S_{12}\right)$. 
Proof. Let us prove the formula $\left[\operatorname{Ind}_{\mathbb{G}_{1}}^{\mathbb{G}_{2}}\left(\mathscr{E}_{1}\right)\left(1_{A_{1}} \otimes S_{12}\right)\right]=\mathscr{E}_{1} \otimes S_{12}$. Fix $\xi \in \mathscr{E}_{1}, s \in S_{12}$ and $\omega \in \mathcal{B}\left(\mathscr{H}_{21}\right)_{*}$. Write $\omega=s^{\prime} \omega^{\prime}$ with $s^{\prime} \in S_{21}$ and $\omega^{\prime} \in \mathcal{B}\left(\mathscr{H}_{21}\right)_{*}$. It follows from $S_{12} \otimes S_{21}=\left[\delta_{11}^{2}\left(S_{11}\right)\left(1_{S_{12}} \otimes S_{21}\right)\right]$ that

$$
\left(\mathrm{id}_{\mathscr{E}_{1} \otimes S_{12}} \otimes \omega\right)\left(\delta_{\mathscr{E}_{1}}^{(2)}(\xi)\right)\left(1_{A_{1}} \otimes s\right)=\left(\mathrm{id}_{\mathscr{\delta}_{1} \otimes S_{12}} \otimes \omega^{\prime}\right)\left(\delta_{\mathscr{E}_{1}}^{(2)}(\xi)\left(1_{\mathscr{E}_{1}} \otimes s \otimes s^{\prime}\right)\right)
$$

is the norm limit of finite sums of elements of the form

$$
\eta=\left(\mathrm{id}_{\mathscr{\odot}_{1}} \otimes \mathrm{id}_{S_{12}} \otimes \omega^{\prime}\right)\left(\delta_{\mathscr{E}_{1}}^{(2)}(\xi)\left(1_{\mathscr{E}_{1}} \otimes \delta_{11}^{2}\left(t^{\prime}\right)\left(1_{S_{12}} \otimes t\right)\right)\right), \quad \text { with } t^{\prime} \in S_{11} \text { and } t \in S_{21} .
$$

It follows from $\left[\delta_{\mathscr{E}_{1}}\left(\mathscr{E}_{1}\right)\left(1_{A_{1}} \otimes S_{11}\right)\right]=\mathscr{E}_{1} \otimes S_{11}$ that

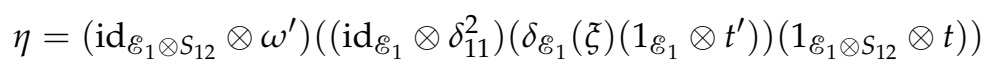

is the norm limit of finite sums of elements of the form

$$
\eta^{\prime}=\left(\mathrm{id}_{\mathscr{E}_{1} \otimes S_{12}} \otimes \omega^{\prime}\right)\left(\zeta \otimes \delta_{11}^{2}\left(t^{\prime \prime}\right)\left(1_{S_{12}} \otimes t\right)\right), \quad \text { with } \zeta \in \mathscr{E}_{1} \text { and } t^{\prime \prime} \in S_{21} .
$$

By using $S_{12}=\left[\left(\operatorname{id}_{S_{12}} \otimes \omega\right)\left(\delta_{11}^{2}(y)\right) ; \omega \in \mathcal{B}\left(\mathscr{H}_{21}\right)_{*}, y \in S_{11}\right]$, we obtain

$$
\eta^{\prime}=\zeta \otimes\left(\operatorname{id}_{S_{12}} \otimes t \omega^{\prime}\right)\left(\delta_{11}^{2}\left(t^{\prime \prime}\right)\right) \in \mathscr{E}_{1} \otimes S_{12} .
$$

Hence, $\left(\operatorname{id}_{\mathscr{E}_{1} \otimes S_{12}} \otimes \omega\right)\left(\delta_{\mathscr{E}_{1}}^{(2)}(\xi)\right)\left(1_{A_{1}} \otimes s\right) \in \mathscr{E}_{1} \otimes S_{12}$ for all $\xi \in \mathscr{E}_{1}, \omega \in \mathcal{B}\left(\mathscr{H}_{21}\right)_{*}$ and $s \in S_{12}$. Therefore, the inclusion

$$
\left[\operatorname{Ind}_{\mathbb{G}_{1}}^{\mathbb{G}_{2}}\left(\mathscr{E}_{1}\right)\left(1_{A_{1}} \otimes S_{12}\right)\right] \subset \mathscr{E}_{1} \otimes S_{12}
$$

is proved. The converse inclusion is obtained by following backwards the above argument. By a similar argument, we prove by using the relation $\left(1_{\mathscr{E}_{1}} \otimes S_{12}\right) \delta_{\mathscr{E}_{1}}\left(\mathscr{E}_{1}\right) \subset \mathscr{E}_{1} \otimes S_{12}$ that $\left(1_{\mathscr{E}_{1}} \otimes S_{12}\right) \operatorname{Ind}_{\mathrm{G}_{1}}^{\mathrm{G}_{2}}\left(\mathscr{E}_{1}\right) \subset \mathscr{E}_{1} \otimes S_{12}$. Hence, $\left[\left(1_{\mathscr{E}_{1}} \otimes S_{12}\right) \operatorname{Ind}_{\mathrm{G}_{1}}^{\mathrm{G}_{2}}\left(\mathscr{E}_{1}\right)\right] \subset \mathscr{E}_{1} \otimes S_{12}$. For the converse inclusion, it suffices to follow backwards the proof as above and to use the continuity of the action $\delta_{\mathscr{E}_{1}}$.

6.3.3 LeMmA. - For all $a \in A_{1}, \xi \in \mathscr{E}_{1}, k \in \mathcal{K}\left(\mathscr{E}_{1}\right)$ and $\omega \in \mathcal{B}\left(\mathscr{H}_{21}\right)_{*}$, we have:

1. $\iota_{A_{1} \otimes S_{11}}\left(\operatorname{id}_{A_{1} \otimes S_{12}} \otimes \omega\right) \delta_{A_{1}}^{(2)}(a)=\left(\operatorname{id}_{J_{1} \otimes S_{12}} \otimes \omega\right) \delta_{J_{1}}^{(2)}\left(\iota_{A_{1}}(a)\right)$;

2. $\iota_{\mathscr{E}_{1} \otimes S_{12}}\left(\mathrm{id}_{\mathscr{E}_{1} \otimes S_{12}} \otimes \omega\right) \delta_{\mathscr{E}_{1}}^{(2)}(\xi)=\left(\operatorname{id}_{J_{1} \otimes S_{12}} \otimes \omega\right) \delta_{J_{1}}^{(2)}\left(\iota_{\mathscr{E}_{1}}(\xi)\right)$;

3. $\iota_{\mathcal{K}\left(\mathscr{E}_{1} \otimes S_{12}\right)}\left(\mathrm{id}_{\mathcal{K}\left(\mathscr{E}_{1}\right) \otimes S_{12}} \otimes \omega\right) \delta_{\mathcal{K}\left(\mathscr{E}_{1}\right)}^{(2)}(k)=\left(\mathrm{id}_{J_{1} \otimes S_{12}} \otimes \omega\right) \delta_{J_{1}}^{(2)}\left(\iota_{\mathcal{K}\left(\mathscr{E}_{1}\right)}(k)\right)$.

Proof. These formulas are straightforward consequences of definitions and the compatibility of $\delta_{J_{1}}$ with $\delta_{A_{1}}$ and $\delta_{\mathscr{E}_{1}}$ and $\delta_{\mathcal{K}\left(\mathscr{E}_{1}\right)}(2.7$ (b), 2.8 (a) [3], 2.3.6.

6.3.4 Proposition. - Let $\operatorname{Ind}_{\mathrm{G}_{1}}^{\mathrm{G}_{2}}\left(A_{1}\right)$ be the induced $C^{*}$-algebra. Then $\operatorname{Ind}_{\mathrm{G}_{1}}^{\mathrm{G}_{2}}\left(\mathscr{E}_{1}\right)$ is a Hilbert $\operatorname{Ind}_{\mathrm{G}_{1}}^{\mathrm{G}_{2}}\left(A_{1}\right)$-module for the right action by composition and the $\operatorname{Ind}_{\mathrm{G}_{1}}^{\mathrm{G}_{2}}\left(A_{1}\right)$-valued inner product given by $\langle\xi, \eta\rangle:=\xi^{*} \circ \eta$ for $\xi, \eta \in \operatorname{Ind}_{\mathbb{G}_{1}}^{\mathbb{G}_{2}}\left(\mathscr{E}_{1}\right)$.

Proof. Let $\omega, \omega^{\prime} \in \mathcal{B}\left(\mathscr{H}_{21}\right)_{*}, a \in A_{1}$ and $\xi \in \mathscr{E}_{1}$. Let $\eta:=\left(\operatorname{id}_{\mathscr{E}_{1} \otimes S_{12}} \otimes \omega\right) \delta_{\mathscr{E}_{1}}^{(2)}(\xi)$ and $x:=\left(\operatorname{id}_{A_{1} \otimes S_{12}} \otimes \omega^{\prime}\right) \delta_{A_{1}}^{(2)}(a)$. We have

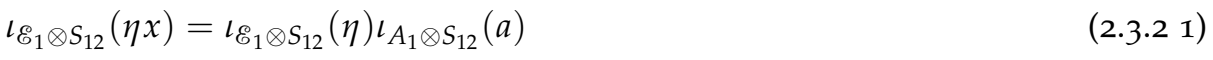

$$
\begin{aligned}
& =\left(\mathrm{id}_{J_{1} \otimes S_{12}} \otimes \omega\right) \delta_{J_{1}}^{(2)}\left(\iota_{\mathbb{E}_{1}}(\xi)\right)\left(\mathrm{id}_{J_{1} \otimes S_{12}} \otimes \omega^{\prime}\right) \delta_{J_{1}}^{(2)}\left(\iota_{A}(a)\right) \\
& =\left(\operatorname{id}_{J_{1} \otimes S_{12}} \otimes \omega \otimes \omega^{\prime}\right)\left(\delta_{J_{1}}^{(2)}\left(\iota_{\mathscr{E}_{1}}(\xi)\right)_{123} \delta_{J_{1}}^{(2)}\left(\iota_{A}(a)\right)_{124}\right) .
\end{aligned}
$$

In virtue of 4.2 a) [2], we have $\iota_{\mathscr{E}_{1} \otimes S_{12}}(\eta x) \in \operatorname{Ind}_{\mathrm{G}_{1}}^{\mathrm{G}_{2}}\left(J_{1}\right)$. Therefore, $\iota_{\mathscr{E}_{1} \otimes S_{12}}(\eta x)$ is the norm limit of finite sums of elements of the form

$$
y=\left(\operatorname{id}_{J_{1} \otimes S_{12}} \otimes \phi\right) \delta_{J_{1}}^{(2)}\left(\begin{array}{cc}
k & \zeta \\
\chi^{*} & b
\end{array}\right) \text {, with } k \in \mathcal{K}\left(\mathscr{E}_{1}\right), \zeta \in \mathscr{E}_{1}, \chi^{*} \in \mathscr{E}_{1}^{*}, b \in A_{1}, \phi \in \mathcal{B}\left(\mathscr{H}_{21}\right)_{*} .
$$


We have 6.3.3

$$
\begin{aligned}
y=\iota_{\mathcal{K}\left(\mathscr{E}_{1} \otimes S_{12}\right)}\left(\mathrm{id}_{\mathcal{K}\left(\mathscr{E}_{1}\right) \otimes}\right. & \left.S_{12} \otimes \phi\right) \delta_{\mathcal{K}\left(\mathscr{E}_{1}\right)}^{(2)}(k)+\iota_{\mathscr{E}_{1} \otimes S_{12}}\left(\mathrm{id}_{\mathscr{E}_{1} \otimes S_{12}} \otimes \phi\right) \delta_{\mathscr{E}_{1}}^{(2)}(\zeta) \\
& +\iota_{\mathscr{E}_{1} \otimes S_{12}}\left(\mathrm{id}_{\mathscr{E}_{1} \otimes S_{12}} \otimes \phi\right) \delta_{\mathscr{E}_{1}}^{(2)}(\chi)^{*}+\iota_{A_{1} \otimes S_{12}}\left(\mathrm{id}_{A_{1} \otimes S_{12}} \otimes \phi\right) \delta_{A_{1}}^{(2)}(b) .
\end{aligned}
$$

By multiplying on the left (resp. right) by $\iota_{\mathcal{K}\left(\mathscr{E}_{1} \otimes S_{12}\right)}\left(1_{\mathscr{E}_{1} \otimes S_{12}}\right)\left(\right.$ resp. $\left.\iota_{A_{1} \otimes S_{12}}\left(1_{A_{1} \otimes S_{12}}\right)\right)$, we obtain (2.3.4) that $\iota_{\mathscr{E}_{1}} \otimes S_{12}(\eta x)$ is the norm limit of finite sums of elements of the form

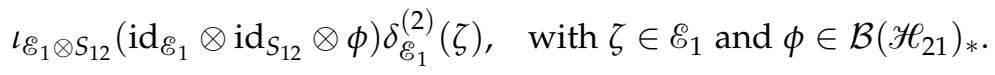

Since $\iota_{\mathscr{E}_{1} \otimes S_{12}}$ is isometric, we have proved that $\eta x \in \operatorname{Ind}_{\mathrm{G}_{1}}^{\mathrm{G}_{2}}\left(\mathscr{E}_{1}\right)$.

Let us prove that $\zeta^{*} \circ \chi \in \operatorname{Ind}_{\mathrm{G}_{1}}^{\mathrm{G}_{2}}\left(A_{1}\right)$ for all $\zeta, \chi \in \operatorname{Ind}_{\mathbb{G}_{1}}^{\mathrm{G}_{2}}\left(\mathscr{E}_{1}\right) \subset \mathcal{L}\left(A_{1} \otimes S_{12}, \mathscr{E}_{1} \otimes S_{12}\right)$. Let us fix $\xi, \eta \in \mathscr{E}_{1}$ and $\omega, \psi \in \mathcal{B}\left(\mathscr{H}_{21}\right)_{*}$. Let us denote $\zeta:=\left(\operatorname{id}_{\mathscr{E}_{1} \otimes S_{12}} \otimes \omega\right) \delta_{\mathscr{E}_{1}}^{(2)}(\xi)$ and $\chi:=\left(\mathrm{id}_{\mathscr{E}_{1} \otimes S_{12}} \otimes \psi\right) \delta_{\mathscr{E}_{1}}^{(2)}(\eta)$. We have

$$
\begin{aligned}
\iota_{A_{1} \otimes S_{12}}\left(\zeta^{*} \circ \chi\right) & =\iota_{\mathscr{E}_{1} \otimes S_{12}}(\zeta)^{*} \iota_{\mathscr{E}_{1} \otimes S_{12}}(\chi) \\
& =\left(\operatorname{id}_{J_{1} \otimes S_{12}} \otimes \omega\right) \delta_{J_{1}}^{(2)}\left(\iota_{\mathscr{E}_{1}}(\xi)^{*}\right)\left(\mathrm{id}_{J_{1} \otimes S_{12}} \otimes \psi\right) \delta_{J_{1}}^{(2)}\left(\iota_{\mathscr{E}_{1}}(\eta)\right) \\
& =\left(\operatorname{id}_{J_{1} \otimes S_{12}} \otimes \omega \otimes \psi\right)\left(\delta_{J_{1}}^{(2)}\left(\iota_{\mathscr{E}_{1}}(\xi)^{*}\right)_{123} \delta_{J_{1}}^{(2)}\left(\iota_{\mathscr{E}_{1}}(\eta)\right)_{124}\right) .
\end{aligned}
$$

Hence, $\iota_{A_{1} \otimes S_{12}}\left(\zeta^{*} \circ \chi\right) \in \operatorname{Ind}_{\mathrm{G}_{1}}^{\mathrm{G}_{2}}\left(J_{1}\right)\left(4.2\right.$ a) [2]). As above, we prove that $\iota_{A_{1} \otimes S_{12}}\left(\zeta^{*} \circ \chi\right)$ is the norm limit of finite sums of elements of the form $\iota_{A_{1} \otimes S_{12}}\left(\mathrm{id}_{A_{1} \otimes S_{12}} \otimes \phi\right) \delta_{A_{1}}^{(2)}(a)$ with $a \in A_{1}$ and $\phi \in \mathcal{B}\left(\mathscr{H}_{21}\right)_{*}$. We have proved that $\zeta^{*} \circ \chi \in \operatorname{Ind}_{\mathbb{G}_{1}}^{\mathbb{G}_{2}}\left(A_{1}\right)$ since $\iota_{A_{1} \otimes S_{12}}$ is isometric.

Let us denote $\left(A_{2}, \delta_{A_{2}}\right):=\operatorname{Ind}_{\mathbb{G}_{1}}^{\mathbb{G}_{2}}\left(A_{1}, \delta_{A_{1}}\right)$ and $\left(J_{2}, \delta_{J_{2}}\right):=\operatorname{Ind}_{\mathbb{G}_{1}}^{\mathbb{G}_{2}}\left(J_{1}, \delta_{J_{1}}\right)$ the induced $\mathbb{G}_{2}-C^{*}$ algebra of $\left(A_{1}, \delta_{A_{1}}\right)$ and $\left(J_{1}, \delta_{J_{1}}\right)$ respectively. We also denote $\mathscr{E}_{2}:=\operatorname{Ind}_{\mathbb{G}_{1}}^{\mathbb{G}_{2}}\left(\mathscr{E}_{1}\right)$ the induced Hilbert $A_{2}$-module as defined above.

In the technical lemma below, we make the identification $\mathcal{M}(A)=\mathcal{L}(A)$. We first recall a well-known corollary of Cohen-Hewitt factorization theorem.

6.3.5 Lemma. - Let $A$ be a $C^{*}$-algebra and $\mathcal{E}$ a Hilbert $A$-module. If $T: A \rightarrow \mathcal{E}$ is a map such that $T(a b)=T(a) b$ for all $a, b \in A$, then $T$ is linear and continuous.

6.3.6 LemMA. - Let $A$ be a $C^{*}$-algebra, $B \subset \mathcal{M}(A)$ a non-degenerate $C^{*}$-subalgebra and $\mathcal{E}$ a Hilbert $A$-module. Let $\mathcal{F} \subset \mathcal{L}(A, \mathcal{E})$ be a Hilbert $B$-module (where $B$ is acting on the right by composition and the B-valued inner product is given by $\left\langle\eta_{1}, \eta_{2}\right\rangle:=\eta_{1}^{*} \circ \eta_{2}$, for all $\eta_{1}, \eta_{2} \in \mathcal{F}$ ) such that $[\mathcal{F} A]=\mathcal{E}$.

(i) There exists a unique map $i: \mathcal{L}(B, \mathcal{F}) \rightarrow \mathcal{L}(A, \mathcal{E})$ such that $i(T)(b a)=(T b)$ a for all $T \in \mathcal{L}(B, \mathcal{F}), b \in B$ and $a \in A$. Moreover, $i$ is an injective linear map whose image is $\operatorname{im}(i)=\left\{S \in \mathcal{L}(A, \mathcal{E}) ; S B \subset \mathcal{F}, S^{*} \mathcal{F} \subset B\right\}$.

(ii) There exists a unique map $j: \mathcal{L}(\mathcal{F} \oplus B) \rightarrow \mathcal{L}(\mathcal{E} \oplus A)$ such that $j(x)(\eta a)=(x \eta)$ a for all $x \in \mathcal{L}(\mathcal{F} \oplus B), \eta \in \mathcal{F} \oplus B$ and $a \in A$. Moreover, $j$ is a unital faithful ${ }^{*}$-homomorphism.

Proof. (i) We have $A=B A$. Let $T \in \mathcal{L}(B, \mathcal{F})$. Let $\left(u_{\lambda}\right)$ be an approximate unit of $B$, we have $(T b) a=\lim _{\lambda}\left[T\left(u_{\lambda} b\right)\right] a=\lim _{\lambda}\left[T\left(u_{\lambda}\right) b\right] a=\lim _{\lambda} T\left(u_{\lambda}\right) b a$, for all $b \in B$ and $a \in A$. In particular, we have $(T b) a=\left(T b^{\prime}\right) a^{\prime}$ for all $b, b^{\prime} \in B$ and $a, a^{\prime} \in A$ such that $b a=b^{\prime} a^{\prime}$. Therefore, $i(T)$ is well defined. Moreover, we have $i(T)\left(a a^{\prime}\right)=(i(T) a) a^{\prime}$ for all $a, a^{\prime} \in A$. Indeed, let us fix $a, a^{\prime} \in A$. Let us write $a=b a^{\prime \prime}$ with $b \in B$ and $a^{\prime \prime} \in A$. We have $i(T)\left(a a^{\prime}\right)=i(T)\left(b\left(a^{\prime \prime} a^{\prime}\right)\right)=(T b) a^{\prime \prime} a^{\prime}=i(T)\left(b a^{\prime \prime}\right) a^{\prime}=(i(T) a) a^{\prime}$. By Lemma 6.3.5. it then follows that $i(T)$ is a bounded linear map. By a straightforward computation, we have $\left\langle i(T)\left(b a^{\prime}\right), \eta a\right\rangle=\left\langle b a^{\prime}, T^{*}(\eta) a\right\rangle$, for all $b \in B, a, a^{\prime} \in A$ and $\eta \in \mathcal{F}$. Hence, $\langle i(T) x, \eta a\rangle=\left\langle x, T^{*}(\eta) a\right\rangle$ for all $x, a \in A$ and $\eta \in \mathcal{F}$. Let $S \in \mathcal{L}(\mathcal{F}, B)$. We have

$$
\left\langle x, \sum_{l=1}^{n} S\left(\eta_{l}\right) a_{l}\right\rangle=\left\langle i\left(S^{*}\right) x, \sum_{l=1}^{n} \eta_{l} a_{l}\right\rangle \text {, for all } a_{1}, \cdots a_{n} \in A, \eta_{1}, \cdots, \eta_{n} \in \mathcal{F} \text { and } x \in A \text {. }
$$


As a consequence, the following map

$$
i^{\prime}(S):\langle\mathcal{F} A\rangle \rightarrow A ; \sum_{l=1}^{n} \eta_{l} a_{l} \mapsto \sum_{l=1}^{n} S\left(\eta_{l}\right) a_{l}
$$

is well-defined and we have $\left\langle x, i^{\prime}(S)(\xi)\right\rangle=\left\langle i\left(S^{*}\right) x, \xi\right\rangle$ for all $\xi \in\langle\mathcal{F} A\rangle$ and $x \in A$. It follows from the boundedness of the linear operator $i\left(S^{*}\right)$ and the Cauchy-Schwarz inequality that $\left\|i^{\prime}(S) \xi\right\|^{2}=\left\|\left\langle i^{\prime}(S) \xi, i^{\prime}(S) \xi\right\rangle\right\|=\left\|\left\langle\xi, i\left(S^{*}\right)\left(i^{\prime}(S) \xi\right)\right\rangle\right\| \leqslant\|\xi\|\left\|i\left(S^{*}\right)\left(i^{\prime}(S) \xi\right)\right\| \leqslant$ $\|\xi\|\left\|i\left(S^{*}\right)\right\|\left\|i^{\prime}(S) \xi\right\|$ for all $\xi \in \mathcal{E}$. Hence, $\left\|i^{\prime}(S) \xi\right\| \leqslant\left\|i\left(S^{*}\right)\right\|\|\xi\|$ for all $\xi \in \mathcal{E}$, which proves the continuity of $i^{\prime}(S)$ since $i^{\prime}(S)$ is linear by definition. In particular, $i^{\prime}(S)$ extends uniquely to a bounded linear map $i^{\prime}(S): \mathcal{E} \rightarrow A$. By continuity of the inner product, we have proved that $i^{\prime}(S) \in \mathcal{L}(\mathcal{E}, A)$ and $i^{\prime}(S)^{*}=i\left(S^{*}\right)$. As a result, we have well-defined maps $i: \mathcal{L}(A, \mathcal{E}) \rightarrow \mathcal{L}(B, \mathcal{F})$ and $i^{\prime}: \mathcal{L}(\mathcal{F}, B) \rightarrow \mathcal{L}(\mathcal{E}, A)$ such that $i(T)^{*}=i^{\prime}\left(T^{*}\right)$ for all $T \in \mathcal{L}(A, \mathcal{E})$. It is clear that $i$ is linear and injective.

It remains to prove that $\operatorname{im}(i)=\left\{S \in \mathcal{L}(A, \mathcal{E}) ; S B \subset \mathcal{F}, S^{*} \mathcal{F} \subset B\right\}$. Let $T \in \mathcal{L}(B, \mathcal{F})$ and $b \in B \subset \mathcal{L}(A)$. For all $a \in A$, we have $[i(T) \circ b] a=i(T)(b a)=(T b) a$. Hence $i(T) \circ b=T(b) \in \mathcal{F}$. Fix $\eta \in \mathcal{F}$. Write $\eta=\zeta b$ with $\zeta \in \mathcal{F}$ and $b \in B$. For all $a \in A$, we have $\left[i(T)^{*} \circ \eta\right] a=\left[i^{\prime}\left(T^{*}\right) \eta\right] a=i^{\prime}\left(T^{*}\right)(\eta a)=i^{\prime}\left(T^{*}\right)(\zeta(b a))=\left(T^{*} \zeta\right) b a=T^{*}(\zeta b) a=T^{*}(\eta) a$. Hence, $i(T)^{*} \circ \eta=T^{*}(\eta) \in B$. Conversely, let us fix $S \in \mathcal{L}(A, \mathcal{E})$ such that $S B \subset \mathcal{F}$ and $S^{*} \mathcal{F} \subset B$. Let $T: A \rightarrow \mathcal{E}$ and $T^{\prime}: \mathcal{F} \rightarrow B$ be the maps defined by:

$$
T(b):=S \circ b, \quad b \in B ; \quad T^{\prime}(\eta):=S^{*} \circ \eta, \quad \eta \in \mathcal{F} .
$$

For all $b \in B$ and $\eta \in \mathcal{F}$, we have $\langle T(b), \eta\rangle=(S \circ b)^{*} \circ \eta=b^{*}\left(S^{*} \circ \eta\right)=\left\langle b, T^{\prime}(\eta)\right\rangle$. Hence $T \in \mathcal{L}(A, \mathcal{E})$ and $T^{*}=T^{\prime}$. Moreover, we have $i(T)(b a)=T(b) a=S(b a)$ for all $a \in A$ and $b \in B$. Thus, we have $S=i(T)$.

(ii) Since $[\mathcal{F} A]=\mathcal{E}$, we have $[(\mathcal{F} \oplus B) A]=\mathcal{E} \oplus A$, which proves the uniqueness of $j$. Let $i_{12}:=i$ and $i_{21}:=i^{\prime}$. By a similar argument as in statement (i), we prove that there exists a unique map $i_{11}: \mathcal{L}(\mathcal{F}) \rightarrow \mathcal{L}(\mathcal{E})$ such that $i_{11}(T)(\eta a)=(T \eta) a$ for all $\eta \in \mathcal{F}$ and $a \in A$. The non-degenerate inclusion of $C^{*}$-algebras $B \subset \mathcal{M}(A)$ extends to a unital *-homomorphism $i_{22}: \mathcal{M}(B) \rightarrow \mathcal{M}(A)$. Then we consider the map $j: \mathcal{L}(\mathcal{F} \oplus B) \rightarrow \mathcal{L}(\mathcal{E} \oplus A)$ defined by $j(x):=\left(i_{k l}\left(x_{k l}\right)\right)_{k, l=1,2}$ for all $x=\left(x_{k l}\right)_{k, l=1,2} \in \mathcal{L}(\mathcal{F} \oplus B)$. It is clear that $j(x)(\eta a)=(x \eta) a$ for all $x \in \mathcal{L}(\mathcal{F} \oplus B), \eta \in \mathcal{F} \oplus B$ and $a \in A$. The fact that $j$ is a unital faithful *homomorphism is then straightforward.

6.3.7 REMARKs. - With the notations and hypotheses of the previous proposition, we have:

(i) for all $T \in \mathcal{L}(B, \mathcal{F}), j\left(\iota_{\mathcal{F}}(T)\right)=\iota_{\mathcal{E}}(i(T))$;

(ii) for all $m \in \mathcal{M}(B), j\left(\iota_{B}(m)\right)=\iota_{A}(m)$, where we identify $\mathcal{M}(B) \subset \mathcal{M}(A)$.

6.3.8 LEMMA. - Let $j=1,2$. We have a canonical embedding

$$
\mathcal{L}\left(A_{2} \otimes S_{2 j}, \mathscr{E}_{2} \otimes S_{2 j}\right) \rightarrow \mathcal{L}\left(A_{1} \otimes S_{12} \otimes S_{2 j}, \mathscr{E}_{1} \otimes S_{12} \otimes S_{2 j}\right) ; T \mapsto \widetilde{T},
$$

where for $T \in \mathcal{L}\left(A_{2} \otimes S_{2 j}, \mathscr{E}_{2} \otimes S_{2 j}\right)$ the operator $\widetilde{T} \in \mathcal{L}\left(A_{1} \otimes S_{12} \otimes S_{2 j}, \mathscr{E}_{1} \otimes S_{12} \otimes S_{2 j}\right)$ is defined by $\widetilde{T}(x a)=T(x)$ a for all $x \in A_{2} \otimes S_{2 j}$ and $a \in A_{1} \otimes S_{12} \otimes S_{2 j}$. Moreover, the image of $\mathcal{L}\left(A_{2} \otimes S_{2 j}, \mathscr{E}_{2} \otimes S_{2 j}\right) \rightarrow \mathcal{L}\left(A_{1} \otimes S_{12} \otimes S_{2 j}, \mathscr{E}_{1} \otimes S_{12} \otimes S_{2 j}\right)$ is

$$
\begin{aligned}
\left\{X \in \mathcal{L}\left(A_{1} \otimes S_{12} \otimes S_{2 j}, \mathscr{E}_{1} \otimes S_{12} \otimes S_{2 j}\right) ; X\left(A_{2} \otimes S_{2 j}\right) \subset \mathscr{E}_{2} \otimes S_{2 j}\right. \text { and } \\
\left.\quad X^{*}\left(\mathscr{E}_{2} \otimes S_{2 j}\right) \subset A_{2} \otimes S_{2 j}\right\} .
\end{aligned}
$$

Proof. This follows from 6.3.6 with $A:=A_{1} \otimes S_{12} \otimes S_{2 j}, B:=A_{2} \otimes S_{2 j}, \mathcal{E}:=\mathscr{E}_{1} \otimes S_{12} \otimes S_{2 j}$ and $\mathcal{F}:=\mathscr{E}_{2} \otimes S_{2 j} \subset \mathcal{L}\left(A_{1} \otimes S_{12} \otimes S_{2 j}, \mathscr{E}_{1} \otimes S_{12} \otimes S_{2 j}\right)$. The assumptions of 6.3.6 are satisfied in this case in virtue of 5.2.5 1 and 6.3.2.

6.3.9 NotAtion. - Let

$$
\mathrm{id}_{\mathscr{E}_{1}} \otimes \delta_{12}^{2}: \mathcal{L}\left(A_{1} \otimes S_{12}, \mathscr{E}_{1} \otimes S_{12}\right) \rightarrow \mathcal{L}\left(A_{1} \otimes S_{12} \otimes S_{22}, \mathscr{E}_{1} \otimes S_{12} \otimes S_{22}\right)
$$

be the unique linear extension of $\mathrm{id}_{\mathscr{E}_{1}} \otimes \delta_{12}^{2}: \mathscr{E}_{1} \otimes S_{12} \rightarrow \mathcal{L}\left(A_{1} \otimes S_{12} \otimes S_{22}, \mathscr{E}_{1} \otimes S_{12} \otimes S_{22}\right)$ such that $\left(\mathrm{id}_{\mathscr{E}_{1}} \otimes \delta_{12}^{2}\right)(T)\left(\mathrm{id}_{A_{1}} \otimes \delta_{12}^{2}\right)(x)=\left(\mathrm{id}_{\mathscr{E}_{1}} \otimes \delta_{12}^{2}\right)(T x)$ for all $x \in \mathcal{M}\left(A_{1} \otimes S_{12}\right)$ and $T \in \mathcal{L}\left(A_{1} \otimes S_{12}, \mathscr{E}_{1} \otimes S_{12}\right)$. 
6.3.10 Proposition-Definition. - There exists a unique linear map

$$
\delta_{\mathscr{E}_{2}}: \mathscr{E}_{2} \rightarrow \mathcal{L}\left(A_{2} \otimes S_{22}, \mathscr{E}_{2} \otimes S_{22}\right)
$$

satisfying the relation $\left[\delta_{\mathscr{E}_{2}}(\xi) a\right] b=\left(\mathrm{id}_{\mathscr{E}_{1}} \otimes \delta_{12}^{2}\right)(\xi)(a b)$ for all $\xi \in \mathscr{E}_{2}, a \in A_{2} \otimes S_{22}$ and $b \in A_{1} \otimes S_{12} \otimes S_{22}$.

Proof. Let us prove the inclusion $\left(\mathrm{id}_{\mathscr{E}_{1}} \otimes \delta_{12}^{2}\right)\left(\mathscr{E}_{2}\right)\left(A_{2} \otimes S_{22}\right) \subset \mathscr{E}_{2} \otimes S_{22}$. It follows from 6.3 .32 that $\iota_{\mathscr{E}_{1} \otimes S_{12}}\left(\mathscr{E}_{2}\right) \subset J_{2}$. Fix $\xi \in \mathscr{E}_{2}$ and $x \in A_{2} \otimes S_{22}$. We have

$$
\begin{aligned}
\iota_{\mathscr{E}_{1} \otimes S_{12} \otimes S_{22}}\left(\left(\mathrm{id}_{\mathscr{E}_{1}} \otimes \delta_{12}^{2}\right)(\xi) x\right) & =\left(\operatorname{id}_{J_{1}} \otimes \delta_{12}^{2}\right)\left(\iota_{\mathscr{E}_{1} \otimes S_{12}}(\xi)\right) \iota_{A_{1} \otimes S_{12} \otimes S_{22}}(x) \\
& =\delta_{J_{2}}\left(\iota_{\mathscr{E}_{1} \otimes S_{12}}(\xi)\right) \iota_{A_{2} \otimes S_{22}}(x) \in J_{2} \otimes S_{22} .
\end{aligned}
$$

As in the proof of 6.3.4. $\iota_{\mathscr{E}_{1} \otimes S_{12} \otimes S_{22}}\left(\left(\mathrm{id}_{\mathscr{\delta}_{1}} \otimes \delta_{12}^{2}\right)(\xi) x\right)$ is the norm limit of finite sums of elements of the form $\iota_{\mathscr{E}_{1}} \otimes S_{12} \otimes S_{22}\left(\left(\mathrm{id}_{\mathscr{E}_{1} \otimes S_{12}} \otimes \omega\right) \delta_{\mathscr{E}_{1}}^{(2)}(\eta) \otimes s\right)$ with $\eta \in \mathscr{E}_{1}, \omega \in \mathcal{B}\left(\mathscr{H}_{21}\right)_{*}$ and $s \in S_{22}$. Hence, $\left(\operatorname{id}_{\mathscr{E}_{1}} \otimes \delta_{12}^{2}\right)(\xi) x \in \mathscr{E}_{2} \otimes S_{22}$ since $\iota_{\mathscr{E}_{1} \otimes S_{12} \otimes S_{22}}$ is isometric. Therefore, we have $\left(\mathrm{id}_{\mathscr{E}_{1}} \otimes \delta_{12}^{2}\right)\left(\mathscr{E}_{2}\right)\left(A_{2} \otimes S_{22}\right) \subset \mathscr{E}_{2} \otimes S_{22}$. The inclusion $\left(\mathrm{id}_{\mathscr{E}_{1}} \otimes \delta_{12}^{2}\right)\left(\mathscr{E}_{2}\right)^{*}\left(\mathscr{E}_{2} \otimes S_{22}\right) \subset A_{2} \otimes S_{22}$ is obtained by a similar argument. Then, the existence and uniqueness of the operator $\delta_{\mathscr{E}_{2}}(\xi) \in \mathcal{L}\left(A_{2} \otimes S_{22}, \mathscr{E}_{2} \otimes S_{22}\right)$ follows as an application of 6.3 .8 with $j=2$. It is clear that the map $\delta_{\mathscr{E}_{2}}: \mathscr{E}_{2} \rightarrow \mathcal{L}\left(A_{2} \otimes S_{22}, \mathscr{E}_{2} \otimes S_{22}\right)$ is linear.

In the following, we prove that $\delta_{\mathscr{E}_{2}}$ is a continuous action of $\mathbb{G}_{2}$ on $\mathscr{E}_{2}$. We also show that the induction procedure for equivariant Hilbert modules is equivalent to that of $\$ 4.3$ [2].

6.3.11 Notations. - Let $e_{1,1}:=\iota_{\mathcal{K}\left(\mathscr{E}_{1}\right)}\left(1_{\mathscr{E}_{1}}\right) \in \mathcal{M}\left(J_{1}\right)$ and $e_{2,1}:=\iota_{A_{1}}\left(1_{A_{1}}\right) \in \mathcal{M}\left(J_{1}\right)$, where we identify $\mathcal{M}\left(J_{1}\right)=\mathcal{L}\left(\mathscr{E}_{1} \oplus A_{1}\right)$. Let $\left(J_{2}, \delta_{J_{2}}, e_{1,2}, e_{2,2}\right)$ be the induced linking $\mathrm{G}_{2}$ $C^{*}$-algebra, with $e_{l, 2}:=e_{l, 1} \otimes 1_{S_{12}} \in \mathcal{M}\left(J_{2}\right)$ for $l=1,2$ (cf. 4.14 [2]). Consider $e_{2,2} J_{2} e_{2,2}$ and $e_{1,2} J_{2} e_{2,2}$ endowed with their structure of $\mathbb{G}_{2}-C^{*}$-algebra and $\mathbb{G}_{2}$-equivariant Hilbert $e_{2,2} J_{2} e_{2,2}$-module [3]. Recall that the morphism $\operatorname{Ind}_{\mathbb{G}_{1}}^{\mathbb{G}_{2}} \iota_{A_{1}}: A_{2} \rightarrow J_{2} ; x \mapsto\left(\iota_{A_{1}} \otimes \mathrm{id}_{S_{12}}\right)(x)$ induces a $\mathrm{G}_{2}$-equivariant ${ }^{*}$-isomormorphism $A_{2} \rightarrow e_{2,2} J_{2} e_{2,2}$ (cf. 4.17, 4.18 [2]).

6.3.12 Proposition. - We use the above notations.

(i) The map $\delta_{\mathscr{E}_{2}}: \mathscr{E}_{2} \rightarrow \mathcal{L}\left(A_{2} \otimes S_{22}, \mathscr{E}_{2} \otimes S_{22}\right)$ is a continuous action of $\mathbb{G}_{2}$ on $\mathscr{E}_{2}$.

(ii) There exists a unique bounded linear map $\operatorname{Ind}_{\mathbb{G}_{1}}^{\mathbb{G}_{2}} l_{\mathscr{E}_{1}}: \mathscr{E}_{2} \rightarrow J_{2}$ such that

$$
\operatorname{Ind}_{\mathbb{G}_{1}}^{\mathrm{G}_{2}} \iota_{\mathscr{E}_{1}}\left(\left(\mathrm{id}_{\mathscr{E}_{1} \otimes S_{12}} \otimes \omega\right) \delta_{\mathscr{E}_{1}}^{(2)}(\xi)\right)=\left(\mathrm{id}_{J_{1} \otimes S_{12}} \otimes \omega\right) \delta_{J_{1}}^{(2)}\left(\iota_{\mathscr{E}_{1}}(\xi)\right),
$$

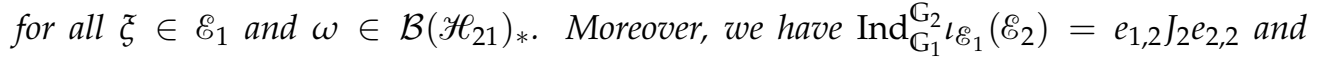
$\operatorname{Ind}_{\mathbb{G}_{1} \iota_{\mathscr{E}_{1}}}^{\mathrm{G}_{2}}$ induces a $\mathbb{G}_{2}$-equivariant unitary equivalence $\mathscr{E}_{2} \rightarrow e_{1,2} J_{2} e_{2,2} ; \xi \mapsto \operatorname{Ind}_{\mathrm{G}_{1}}^{\mathrm{G}_{2}} \mathscr{E}_{1}(\xi)$ over the $\mathbb{G}_{2}$-equivariant ${ }^{*}$-isomorphism $A_{2} \rightarrow e_{2,2} J_{2} e_{2,2} ; a \mapsto \operatorname{Ind}_{\mathbb{G}_{1}}^{\mathrm{G}_{2}} \iota_{A_{1}}(a)$.

(iii) There exists a unique *-homomorphism $\tau: \mathcal{K}\left(\mathscr{E}_{2} \oplus A_{2}\right) \rightarrow J_{2}$ such that $\tau \circ \iota_{\mathscr{E}_{2}}=\operatorname{Ind}_{\mathrm{G}_{1}}^{\mathrm{G}_{2}} \iota_{\mathscr{E}_{1}}$ and $\tau \circ \iota_{A_{2}}=\operatorname{Ind}_{\mathbb{G}_{1} l_{2} \iota_{1}}^{\mathbb{G}_{2}}$. Moreover, $\tau$ is an isomorphism of linking $\mathbb{G}_{2}-C^{*}$-algebras.

(iv) If $T \in \operatorname{Ind}_{\mathrm{G}_{1}}^{\mathrm{G}_{2}}\left(\mathcal{K}\left(\mathscr{E}_{1}\right)\right) \subset \mathcal{L}\left(\mathscr{E}_{1} \otimes S_{12}\right)$ and $\eta \in \mathscr{E}_{2} \subset \mathcal{L}\left(A_{1} \otimes S_{12}, \mathscr{E}_{1} \otimes S_{12}\right)$, then we have $T \circ \eta \in \mathscr{E}_{2}$. Moreover, for all $T \in \operatorname{Ind}_{\mathrm{G}_{1}}^{\mathrm{G}_{2}}\left(\mathcal{K}\left(\mathscr{E}_{1}\right)\right)$, we have $[\eta \mapsto T \circ \eta] \in \mathcal{K}\left(\mathscr{E}_{2}\right)$. More precisely, the map $\operatorname{Ind}_{\mathrm{G}_{1}}^{\mathrm{G}_{2}}\left(\mathcal{K}\left(\mathscr{E}_{1}\right)\right) \rightarrow \mathcal{K}\left(\mathscr{E}_{2}\right) ; T \mapsto[\eta \mapsto T \circ \eta]$ is a $\mathrm{G}_{2}$-equivariant *-isomorphism.

Proof. Let us denote $B:=e_{2,2} J_{2} e_{2,2}$ and $\mathscr{F}:=e_{1,2} J_{2} e_{2,2}$ for short.

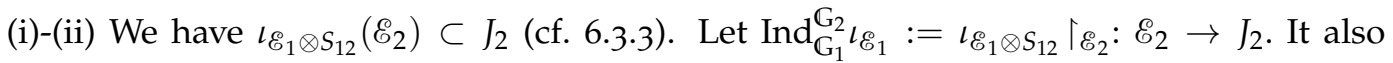
follows from the formulas $\delta_{J_{1}}^{(2)}\left(e_{l, 1}\right)=e_{l, 2} \otimes 1_{S_{21}}$ for $l=1,2$ (4.14 [2]) that

$$
\begin{aligned}
e_{1,2} J_{2} e_{2,2} & =\left[\left(\operatorname{id}_{J_{1} \otimes S_{12}} \otimes \omega\right) \delta_{J_{1}}^{(2)}\left(e_{1,1} x e_{2,1}\right) ; x \in J_{1}, \omega \in \mathcal{B}\left(\mathscr{H}_{21}\right)_{*}\right] \\
& =\left[\left(\operatorname{id}_{J_{1} \otimes S_{12}} \otimes \omega\right) \delta_{J_{1}}^{(2)}\left(\iota_{\mathscr{E}_{1}}(\xi)\right) ; \xi \in \mathscr{E}_{1}, \omega \in \mathcal{B}\left(\mathscr{H}_{21}\right)_{*}\right] \\
& =\operatorname{Ind}_{\mathbb{G}_{1} \mathbb{E}_{1}}^{G_{2}}\left(\mathscr{E}_{2}\right) .
\end{aligned}
$$


Let $\xi, \eta \in \mathscr{E}_{2}$. Since $\langle\xi, \eta\rangle \in A_{2}$, we have (cf. 2.3.2 3 )

$$
\left\langle\operatorname{Ind}_{\mathrm{G}_{1}}^{\mathrm{G}_{2}} \iota_{\mathscr{E}_{1}}(\xi), \operatorname{Ind}_{\mathrm{G}_{1}}^{\mathrm{G}_{2}} \iota_{\mathscr{E}_{1}}(\eta)\right\rangle=\iota_{\mathscr{E}_{1} \otimes S_{12}}(\xi)^{*} \iota_{\mathscr{E}_{1} \otimes S_{12}}(\eta)=\iota_{A_{1} \otimes S_{12}}(\langle\xi, \eta\rangle)=\operatorname{Ind}_{\mathbb{G}_{1}}^{\mathrm{G}_{2}} \iota_{A_{1}}(\langle\xi, \eta\rangle) .
$$

We also have $\operatorname{Ind}_{\mathbb{G}_{1} \mathscr{E}_{1}}^{\mathbb{G}_{2}}(\xi)=\operatorname{Ind}_{\mathbb{G}_{1} \mathscr{E}_{1}}^{\mathrm{G}_{2}}(\xi) \operatorname{Ind}_{\mathbb{G}_{1}}^{\mathrm{G}_{2}} l_{A_{1}}(a)$ for all $a \in A_{2}$ and $\xi \in \mathscr{E}_{2}$ (cf. 2.3.2 1 ). The map $\Phi: \mathscr{E}_{2} \rightarrow \mathscr{F} ; \xi \mapsto \operatorname{Ind}_{\mathbb{G}_{1} \mathscr{E}_{1}}^{\mathbb{G}_{2}}(\xi)$ is a unitary equivalence of Hilbert modules over the *-isomorphism $\phi: A_{2} \rightarrow B ; a \mapsto \operatorname{Ind}_{\mathbb{G}_{1}}^{\mathbb{G}_{2}} l_{A_{1}}(a)$.

Let us prove that $\left(\Phi \otimes \mathrm{id}_{S_{22}}\right) \circ \delta_{\mathscr{E}_{2}}=\delta_{\mathscr{F}} \circ \Phi$. It is immediately verified that for $\xi \in \mathscr{E}_{1} \otimes S_{12}$, the formula $\left(\iota_{\mathscr{E}_{1} \otimes S_{12}} \otimes \mathrm{id}_{S_{22}}\right)\left(\mathrm{id}_{\mathscr{E}_{1}} \otimes \delta_{12}^{2}\right)(\xi)=\left(\mathrm{id}_{J_{1}} \otimes \delta_{12}^{2}\right)\left(\iota_{\mathscr{E}_{1} \otimes S_{12}}(\xi)\right)$ holds true. Let us fix $\xi \in \mathcal{L}\left(A_{1} \otimes S_{12}, \mathscr{E}_{1} \otimes S_{12}\right)$. For all $a \in A_{1} \otimes S_{12}$ and $x \in A_{1} \otimes S_{12} \otimes S_{22}$, we have

$$
\begin{aligned}
\iota_{\mathscr{ஜ}_{1} \otimes S_{12} \otimes S_{22}}\left(\left(\mathrm{id}_{\mathscr{\Xi}_{1}} \otimes \delta_{12}^{2}\right)(\xi)\right) \iota_{A_{1} \otimes S_{12} \otimes S_{22}}\left(\left(\mathrm{id}_{A_{1}} \otimes \delta_{12}^{2}\right)(a) x\right) \\
=\left(\operatorname{id}_{J_{1}} \otimes \delta_{12}^{2}\right)\left(\iota_{\mathscr{E}_{1} \otimes S_{12}}(\xi a)\right) \iota_{A_{1} \otimes S_{12} \otimes S_{22}}(x)
\end{aligned}
$$

and $\left(\mathrm{id}_{J_{1}} \otimes \delta_{12}^{2}\right)\left(\iota_{\mathscr{E}_{1} \otimes S_{12}}(\xi a)\right)=\left(\mathrm{id}_{J_{1}} \otimes \delta_{12}^{2}\right)\left(\iota_{\mathscr{E}_{1} \otimes S_{12}}(\xi)\right) \iota_{A_{1} \otimes S_{12} \otimes S_{22}}\left(\left(\mathrm{id}_{A_{1}} \otimes \delta_{12}^{2}\right)(a)\right)$. Hence,

$$
\begin{aligned}
\iota_{\mathscr{E}_{1} \otimes S_{12} \otimes S_{22}}\left(\left(\mathrm{id}_{\mathscr{E}_{1}} \otimes \delta_{12}^{2}\right)(\xi)\right) \iota_{A_{1} \otimes S_{12} \otimes S_{22}}\left(\left(\mathrm{id}_{A_{1}} \otimes \delta_{12}^{2}\right)(a) x\right) \\
\quad=\left(\operatorname{id}_{J_{1}} \otimes \delta_{12}^{2}\right)\left(\iota_{\mathscr{E}_{1} \otimes S_{12}}(\xi)\right) \iota_{A_{1} \otimes S_{12} \otimes S_{22}}\left(\left(\operatorname{id}_{A_{1}} \otimes \delta_{12}^{2}\right)(a) x\right) .
\end{aligned}
$$

Thus, $\iota_{\mathscr{E}_{1} \otimes S_{12} \otimes S_{22}}\left(\left(\mathrm{id}_{\mathscr{E}_{1}} \otimes \delta_{12}^{2}\right)(\xi)\right) \iota_{A_{1} \otimes S_{12} \otimes S_{22}}(x)=\left(\operatorname{id}_{J_{1}} \otimes \delta_{12}^{2}\right)\left(\iota_{\mathscr{E}_{1} \otimes S_{12}}(\xi)\right) \iota_{A_{1} \otimes S_{12} \otimes S_{22}}(x)$ for all $\xi \in \mathcal{L}\left(A_{1} \otimes S_{12}, \mathscr{E}_{1} \otimes S_{12}\right)$ and $a \in A_{1} \otimes S_{12} \otimes S_{22}$ in virtue of the non-degeneracy of $\operatorname{id}_{A_{1}} \otimes \delta_{12}^{2}$. Let us fix $\xi \in \mathscr{E}_{2}$. For all $x \in A_{2} \otimes S_{22}$ and $y \in A_{1} \otimes S_{12} \otimes S_{21}$ we have

$$
\begin{aligned}
{\left[\left(\Phi \otimes \operatorname{id}_{S_{22}}\right) \delta_{\mathscr{E}_{2}}(\xi)\left(\phi \otimes \operatorname{id}_{S_{22}}\right)(x)\right] \iota_{A_{1} \otimes S_{12} \otimes S_{22}}(y) } & =\iota_{\mathscr{E}_{1} \otimes S_{12} \otimes S_{22}}\left(\left(\delta_{\mathscr{E}_{2}}(\xi) x\right) y\right) \\
& =\iota_{\mathscr{E}_{1} \otimes S_{12} \otimes S_{22}}\left(\left(\mathrm{id}_{\mathscr{E}_{1}} \otimes \delta_{12}^{2}\right)(\xi)(x y)\right) \\
& =\left(\operatorname{id}_{J_{1}} \otimes \delta_{12}^{2}\right)\left(\iota_{\mathscr{E}_{1} \otimes S_{12}}(\xi)\right) \iota_{A_{1} \otimes S_{12} \otimes S_{22}}(x y) \\
& =\left[\delta_{\mathscr{F}}(\Phi(\xi))\left(\phi \otimes \operatorname{id}_{S_{22}}\right)(x)\right] \iota_{A_{1} \otimes S_{12} \otimes S_{22}}(y),
\end{aligned}
$$

which also holds for all $y \in \mathcal{M}\left(A_{1} \otimes S_{12} \otimes S_{22}\right)$ by strict continuity. In particular, by applying this formula for $y \in A_{2} \otimes S_{22}$, we have then proved that

$$
\left(\Phi \otimes \operatorname{id}_{S_{22}}\right)\left(\delta_{\mathscr{E}_{2}}(\xi)\right)\left(\phi \otimes \operatorname{id}_{S_{22}}\right)(x)=\delta_{\mathscr{F}}(\Phi(\xi))\left(\phi \otimes \operatorname{id}_{S_{22}}\right)(x)
$$

for all $x \in A_{2} \otimes S_{22}$. Hence, $\left(\Phi \otimes \mathrm{id}_{S_{22}}\right)\left(\delta_{\mathscr{E}_{2}}(\xi)\right)=\delta_{\mathscr{F}}(\Phi(\xi))$ for all $\xi \in \mathscr{E}_{2}$. This proves that $\delta_{\mathscr{E}_{2}}$ is a continuous action of $\mathrm{G}_{2}$ on $\mathscr{E}_{2}$ and $\Phi$ is $\mathbb{G}_{2}$-equivariant.

(iii) There exists a unique unital faithful ${ }^{*}$-homomorphism

$$
j: \mathcal{L}\left(\mathscr{E}_{2} \oplus A_{2}\right) \rightarrow \mathcal{L}\left(\left(\mathscr{E}_{1} \otimes S_{12}\right) \oplus\left(A_{1} \otimes S_{12}\right)\right)
$$

such that $j(x)(\eta a)=(x \eta) a$ for all $x \in \mathcal{L}\left(\mathscr{E}_{2} \oplus A_{2}\right), \eta \in \mathscr{E}_{2} \oplus A_{2}$ and $a \in A_{1} \otimes S_{12}$ with $A:=A_{1} \otimes S_{12}, B:=A_{2}, \mathcal{E}:=\mathscr{E}_{1} \otimes S_{12}$ and $\left.\mathcal{F}:=\mathscr{E}_{2}\right)$. Now, it should be noted that we have the following canonical identifications

$$
J_{2} \subset \mathcal{M}\left(J_{1} \otimes S_{12}\right)=\mathcal{L}\left(\left(\mathscr{E}_{1} \oplus A_{1}\right) \otimes S_{12}\right)=\mathcal{L}\left(\left(\mathscr{E}_{1} \otimes S_{12}\right) \oplus\left(A_{1} \otimes S_{12}\right)\right) .
$$

We have $j\left(\iota_{\mathscr{E}_{2}}(\xi)\right)=\operatorname{Ind}_{\mathrm{G}_{1}}^{\mathrm{G}_{2}} \iota_{\mathscr{E}_{1}}(\xi)$ for all $\xi \in \mathscr{E}_{2}$ and $j\left(\iota_{A_{2}}(b)\right)=\operatorname{Ind}_{\mathrm{G}_{1}}^{\mathrm{G}_{2}} \iota_{A_{1}}(b)$ for all $b \in A_{2}$ (cf. 6.3.7). In particular, we have $j\left(\mathcal{K}\left(\mathscr{E}_{2} \oplus A_{2}\right)\right) \subset J_{2}$. Let $\tau:=\left.j\right|_{\mathcal{K}\left(\mathscr{E}_{2} \oplus A_{2}\right)}: \mathcal{K}\left(\mathscr{E}_{2} \oplus A_{2}\right) \rightarrow J_{2}$. Since $J_{2}$ is generated by $e_{1,2} J_{2} e_{2,2}$ and $e_{2,2} J_{2} e_{2,2}$ as a $C^{*}$-algebra, $\tau$ has dense range (cf. (ii)); moreover, $\tau$ is also isometric (faithful), therefore $\tau$ is surjective. Thus, we have proved that $\tau$ is a ${ }^{*}$-isomorphism. The $G_{2}$-equivariance of $\tau$ is derived from straightforward computations.

(iv) Consider the $\mathbb{G}_{2}$-equivariant *-isomorphism

$$
\varphi: \operatorname{Ind}_{\mathrm{G}_{1}}^{\mathrm{G}_{2}}\left(\mathcal{K}\left(\mathscr{E}_{1}\right)\right) \rightarrow e_{1,2} J_{2} e_{1,2} ; k \mapsto \operatorname{Ind}_{\mathrm{G}_{1}}^{\mathrm{G}_{2}} \iota \mathcal{K}\left(\mathscr{E}_{1}\right)(k)
$$


(cf. 4.18 [2], note that $\left.\mathcal{K}(\mathscr{F})=e_{1,2} J_{2} e_{1,2}\right)$. By statement (ii), $\tau$ induces by restriction a $\mathbb{G}_{2}-$ equivariant ${ }^{*}$-isomorphism $\tau: f_{1,2} \mathcal{K}\left(\mathscr{E}_{2} \oplus A_{2}\right) f_{1,2} \rightarrow e_{1,2} J_{2} e_{1,2}$, where $f_{1,2}:=\iota_{\mathscr{E}_{2}}\left(1_{\mathscr{E}_{2}}\right)$ and $f_{2,2}:=\iota_{A_{2}}\left(1_{A_{2}}\right)$. We have an isomorphism $\psi: \mathcal{K}\left(\mathscr{E}_{2}\right) \rightarrow f_{1,2} \mathcal{K}\left(\mathscr{E}_{2} \oplus A_{2}\right) f_{1,2} ; k \mapsto \iota_{\mathcal{K}\left(\mathscr{E}_{2}\right)}(k)$ of $\mathbb{G}_{2}$-C*-algebras. Hence, $\chi:=\psi^{-1} \circ \tau^{-1} \circ \varphi: \operatorname{Ind}_{\mathbb{G}_{1}}^{\mathrm{G}_{2}}\left(\mathcal{K}\left(\mathscr{E}_{1}\right)\right) \rightarrow \mathcal{K}\left(\mathscr{E}_{2}\right)$ is an isomorphism of $\mathbb{G}_{2}$-C $\mathrm{C}^{*}$-algebras. It is clear that $\chi(T) \xi=T \circ \xi$ for all $T \in \operatorname{Ind}_{\mathrm{G}_{1}}^{\mathrm{G}_{2}}\left(\mathcal{K}\left(\mathscr{E}_{1}\right)\right) \subset \mathcal{L}\left(\mathscr{E}_{1} \otimes S_{12}\right)$ and $\xi \in \mathscr{E}_{2} \subset \mathcal{L}\left(A_{1} \otimes S_{12}, \mathscr{E}_{1} \otimes S_{12}\right)$.

6.3.13 Proposition-Definition. - Let us fix some notations. Consider:

- two $\mathrm{G}_{1}-C^{*}$-algebras $A_{1}$ and $B_{1}$;

- two $\mathrm{G}_{1}$-equivariant Hilbert modules $\mathscr{E}_{1}$ and $\mathscr{F}_{1}$ over $A_{1}$ and $B_{1}$ respectively;

- a $\mathbb{G}_{1}$-equivariant unitary equivalence $\Phi_{1}: \mathscr{E}_{1} \rightarrow \mathscr{F}_{1}$ over a $\mathbb{G}_{1}$-equivariant ${ }^{*}$-isomorphism $\phi_{1}: A_{1} \rightarrow B_{1}$.

Denote by:

- $A_{2}:=\operatorname{Ind}_{\mathbb{G}_{1}}^{\mathbb{G}_{2}}\left(A_{1}\right)$ and $B_{2}:=\operatorname{Ind}_{\mathbb{G}_{1}}^{\mathbb{G}_{2}}\left(B_{1}\right)$ the induced $\mathbb{G}_{2}-C^{*}$-algebras;

- $\operatorname{Ind}_{\mathbb{G}_{1}}^{\mathbb{G}_{2}}\left(\phi_{1}\right): A_{2} \rightarrow B_{2}$ the induced $\mathbb{G}_{2}$-equivariant ${ }^{*}$-isomorphism;

- $\mathscr{E}_{2}:=\operatorname{Ind}_{\mathbb{G}_{1}}^{\mathrm{G}_{2}}\left(\mathscr{E}_{1}\right)$ and $\mathscr{F}_{2}:=\operatorname{Ind}_{\mathbb{G}_{1}}^{\mathrm{G}_{2}}\left(\mathscr{F}_{1}\right)$ the induced $\mathbb{G}_{2}$-equivariant Hilbert modules over $A_{2}$ and $B_{2}$ respectively;

- $\Phi_{1} \otimes \operatorname{id}_{S_{12}}: \mathcal{L}\left(A_{1} \otimes S_{12}, \mathscr{E}_{1} \otimes S_{12}\right) \rightarrow \mathcal{L}\left(B_{1} \otimes S_{12}, \mathscr{F}_{1} \otimes S_{12}\right)$ the unique linear map such that $\left(\Phi_{1} \otimes \operatorname{id}_{S_{12}}\right)(T)\left(\phi_{1} \otimes \mathrm{id}_{S_{12}}\right)(x)=\left(\Phi_{1} \otimes \mathrm{id}_{S_{12}}\right)(T x)$ for all $\mathcal{L}\left(A_{1} \otimes S_{12}, \mathscr{E}_{1} \otimes S_{12}\right)$ and $x \in A_{1} \otimes S_{12}$ (cf. 8.3.6).

Then, $\left(\Phi_{1} \otimes \operatorname{id}_{S_{12}}\right)\left(\mathscr{E}_{2}\right) \subset \mathscr{F}_{2}$ and the map $\operatorname{Ind}_{\mathbb{G}_{1}}^{\mathrm{G}_{2}}\left(\Phi_{1}\right):=\left(\Phi_{1} \otimes \mathrm{id}_{S_{12}}\right) \Gamma_{\mathscr{E}_{2}}: \mathscr{E}_{2} \rightarrow \mathscr{F}_{2}$ is a $\mathbb{G}_{2}$-equivariant unitary equivalence over $\operatorname{Ind}_{\mathbb{G}_{1}}^{\mathrm{G}_{2}}\left(\phi_{1}\right): A_{2} \rightarrow B_{2}$. Moreover, for all $\xi \in \mathscr{E}_{1}$ and $\omega \in \mathcal{B}\left(\mathscr{H}_{21}\right)_{*}$ we have $\operatorname{Ind}_{\mathbb{G}_{1}}^{\mathbb{G}_{2}}\left(\Phi_{1}\right)\left(\left(\mathrm{id}_{\mathscr{E}_{1} \otimes S_{12}} \otimes \omega\right) \delta_{\mathscr{E}_{1}}^{(2)}(\xi)\right)=\left(\operatorname{id}_{\mathscr{F}_{1} \otimes S_{12}} \otimes \omega\right) \delta_{\mathscr{F}_{1}}^{(2)}\left(\Phi_{1} \xi\right)$.

Proof. Denote by $J_{1}:=\mathcal{K}\left(\mathscr{E}_{1} \oplus A_{1}\right)$ and $K_{1}:=\mathcal{K}\left(\mathscr{F}_{1} \oplus B_{1}\right)$ the linking $\mathbb{G}_{1}$ - $C^{*}$-algebras, whose linking structures are respectively defined by: $e_{1,1}:=\iota_{\mathscr{E}_{1}}\left(1_{\mathscr{E}_{1}}\right), e_{2,1}:=\iota_{A_{1}}\left(1_{A_{1}}\right)$; $f_{1,1}:=\iota_{\mathscr{F}_{1}}\left(1_{\mathscr{F}_{1}}\right), f_{2,1}:=\iota_{B_{1}}\left(1_{B_{1}}\right)$. We also denote by $\left(J_{2}, \delta_{J_{2}}, e_{1,2}, e_{2,2}\right)$ and $\left(K_{2}, \delta_{K_{2}}, f_{1,2}, f_{2,2}\right)$ the induced linking $\mathbb{G}_{2}-C^{*}$-algebras, where $e_{l, 2}:=e_{l, 1} \otimes 1_{S_{12}}$ and $f_{l, 2}:=f_{l, 1} \otimes 1_{S_{12}}$ for $l=1,2$ (cf. 4.14 [2]). There exists a unique ${ }^{*}$-isomorphism $\tau_{1}: J_{1} \rightarrow K_{1}$ such that $\tau_{1} \circ \iota_{\mathscr{E}_{1}}=\iota_{\mathscr{F}_{1}} \circ \Phi_{1}$ and $\tau_{1} \circ \iota_{A_{1}}=\iota_{B_{1}} \circ \phi_{1}$ (cf. 8.3.5 and 6.1.18. We then denote by

$$
\tau_{2}:=\operatorname{Ind}_{\mathrm{G}_{1}}^{\mathrm{G}_{2}} \tau_{1}: J_{2} \rightarrow K_{2}
$$

the induced morphism. Since $\tau_{2}$ is an isomorphism of linking $\mathrm{G}_{2}-\mathrm{C}^{*}$-algebras, it induces a $G_{2}$-equivariant unitary equivalence $\Psi: e_{1,2} J_{2} e_{2,2} \rightarrow f_{1,2} K_{2} f_{2,2}$ over the isomorphism of $\mathbb{G}_{2}-C^{*}$-algebras $\psi: e_{2,2} J_{2} e_{2,2} \rightarrow f_{2,2} K_{2} f_{2,2}$. Since $\tau_{1} \circ \iota_{A_{1}}=\iota_{B_{1}} \circ \phi_{1}$, we have

$$
\tau_{2} \circ \operatorname{Ind}_{\mathrm{G}_{1}}^{\mathrm{G}_{2}} \iota_{A_{1}}=\operatorname{Ind}_{\mathrm{G}_{1}}^{\mathrm{G}_{2} \iota_{B_{1}} \circ \phi_{2}} \text {. }
$$

Therefore, by composition of $\mathrm{G}_{2}$-equivariant unitary equivalences (cf. 8.3.2 2) and by applying 6.3.12, we obtain a $\mathbb{G}_{2}$-equivariant $\phi_{2}$-compatible unitary operator $\Phi_{2}: \mathscr{E}_{2} \rightarrow \mathscr{F}_{2}$. By a straightforward computation, we show that $\Phi_{2}=\left.\left(\Phi_{1} \otimes \mathrm{id}_{S_{12}}\right)\right|_{\mathscr{E}_{2}}$.

By exchanging the roles of $G_{1}$ and $G_{2}$, we define as above an induction procedure for $\mathrm{G}_{2}$-equivariant Hilbert modules.

In the following, we investigate the composition of $\operatorname{Ind}_{\mathrm{G}_{1}}^{\mathbb{G}_{2}}$ and $\operatorname{Ind}_{\mathrm{G}_{2}}^{\mathrm{G}_{1}}$. Let $A_{1}$ be a $\mathrm{G}_{1}-\mathrm{C}^{*}$ algebra and $\mathscr{E}_{1}$ a $\mathbb{G}_{1}$-equivariant Hilbert $A_{1}$-module. Denote by:

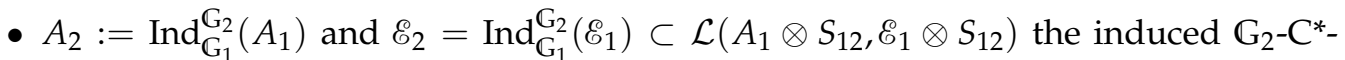
algebra and the induced $\mathbb{G}_{2}$-equivariant Hilbert $A_{2}$-module; 
- $C=\operatorname{Ind}_{\mathrm{G}_{2}}^{\mathrm{G}_{1}}\left(A_{2}\right)$ and $\mathscr{F}:=\operatorname{Ind}_{\mathrm{G}_{2}}^{\mathrm{G}_{1}}\left(\mathscr{E}_{2}\right) \subset \mathcal{L}\left(A_{2} \otimes S_{21}, \mathscr{E}_{2} \otimes S_{21}\right)$ the induced $\mathrm{G}_{1}-\mathrm{C}^{*}$ algebra and the induced $\mathrm{G}_{1}$-equivariant Hilbert $C$-module.

6.3.14 Proposition. - With the above notations and hypotheses, we have the following statements:

1. there exists a unique map $\Pi_{1}: \mathscr{E}_{1} \rightarrow \mathscr{F}$ such that

$$
\left(\Pi_{1}(\xi) x\right) a=\delta_{\mathscr{E}_{1}}^{(2)}(\xi)(x a), \quad \text { for all } \xi \in \mathscr{E}_{1}, x \in A_{2} \otimes S_{21} \text { and } a \in A_{1} \otimes S_{12} \otimes S_{21} ;
$$

moreover, $\Pi_{1}$ is a $\mathbb{G}_{1}$-equivariant unitary equivalence over the $\mathbb{G}_{1}$-equivariant ${ }^{*}$-isomorphism $\pi_{1}: A_{1} \rightarrow C ; a \mapsto \delta_{A_{1}}^{(2)}(a) ;$

2. $\delta_{\mathscr{E}_{1}}^{2}: \mathscr{E}_{1} \rightarrow \widetilde{\mathcal{M}}\left(\mathscr{E}_{2} \otimes S_{21}\right) ; \xi \mapsto \Pi_{1}(\xi)$ is a well-defined linear map such that:

(i) $\delta_{\mathscr{E}_{1}}^{2}(\xi a)=\delta_{\mathscr{E}_{1}}^{2}(\xi) \delta_{A_{1}}^{2}(a)$ and $\left\langle\delta_{\mathscr{E}_{1}}^{2}(\xi), \delta_{\mathscr{E}_{1}}^{2}(\eta)\right\rangle=\delta_{A_{1}}^{2}(\langle\xi, \eta\rangle)$ for all $\xi, \eta \in \mathscr{E}_{1}$ and $a \in A_{1}$

(ii) $\left[\delta_{\mathscr{E}_{1}}^{2}\left(\mathscr{E}_{1}\right)\left(1_{A_{2}} \otimes S_{21}\right)\right]=\mathscr{E}_{2} \otimes S_{21}=\left[\left(1_{\mathscr{E}_{2}} \otimes S_{21}\right) \delta_{\mathscr{E}_{1}}^{2}\left(\mathscr{E}_{1}\right)\right]$.

Proof. 1. The existence and uniqueness of $\Pi_{1}$ is an immediate application of 6.3 .8 with $j=1$ and the proof is very similar to that of 6.3.10 The fact that $\Pi_{1}$ is a $\mathbb{G}_{1}$-equivariant unitary equivalence over $\pi_{1}$ is a straightforward consequence of 6.3.12 (ii), (iii) and 5.2.6 2 . 2. Statement (ii) and the fact that $\delta_{\mathscr{E}_{1}}^{2}$ takes its values in $\widetilde{\mathcal{M}}\left(\mathscr{E}_{2} \otimes S_{21}\right)$ are proved by combining the formulas $\left[\mathscr{F}\left(1_{A_{2}} \otimes S_{21}\right)\right]=\mathscr{E}_{2} \otimes S_{21}=\left[\left(1_{\mathscr{E}_{2}} \otimes S_{21}\right) \mathscr{F}\right]$ (cf. 6.3.2) with the fact that $\Pi_{1}$ is bijective. Statement (i) follows from the compatibility of $\Pi_{1}$ with $\pi_{1}$.

We have proved the following result:

6.3.15 THEOREM. - Let $\mathrm{G}_{1}$ and $\mathrm{G}_{2}$ be two monoidally equivalent regular locally compact quantum groups. The map

$$
\operatorname{Ind}_{\mathbb{G}_{1}}^{\mathrm{G}_{2}}:\left(\mathscr{E}_{1}, \delta_{\mathscr{E}_{1}}\right) \mapsto\left(\mathscr{E}_{2}:=\operatorname{Ind}_{\mathrm{G}_{1}}^{\mathrm{G}_{2}}\left(\mathscr{E}_{1}\right), \delta_{\mathscr{E}_{2}}: \xi \in \mathscr{E}_{2} \mapsto\left[x \in A_{2} \otimes S_{22} \mapsto\left(\mathrm{id}_{\mathscr{E}_{1}} \otimes \delta_{12}^{2}\right)(\xi) x\right]\right),
$$

where $\mathscr{E}_{1}$ is a Hilbert module over the $\mathbb{G}_{1}-C^{*}$-algebra $A_{1}$ and $A_{2}=\operatorname{Ind}_{\mathbb{G}_{1}}^{\mathbb{G}_{2}}\left(A_{1}\right)$ denotes the induced $\mathrm{G}_{2}-C^{*}$-algebra, is a one-to-one correspondence up to unitary equivalence. The inverse map, up to unitary equivalence, is

$$
\operatorname{Ind}_{\mathrm{G}_{2}}^{\mathrm{G}_{1}}:\left(\mathscr{F}_{2}, \delta_{\mathscr{F}_{2}}\right) \mapsto\left(\mathscr{F}_{1}:=\operatorname{Ind}_{\mathrm{G}_{2}}^{\mathrm{G}_{1}}\left(\mathscr{F}_{2}\right), \delta_{\mathscr{F}_{1}}: \xi \in \mathscr{F}_{1} \mapsto\left[x \in B_{1} \otimes S_{11} \mapsto\left(\operatorname{id}_{\mathscr{F}_{2}} \otimes \delta_{21}^{1}\right)(\xi) x\right]\right),
$$

where $\mathscr{F}_{2}$ is a Hilbert module over the $\mathrm{G}_{2}-C^{*}$-algebra $B_{2}$ and $B_{1}=\operatorname{Ind}_{\mathrm{G}_{1}}^{\mathrm{G}_{2}}\left(B_{2}\right)$ denotes the induced $\mathrm{G}_{1}-C^{*}$-algebra.

Proof. This is a consequence of Propositions 6.3.14, 6.3.13 and the corresponding results obtained by exchanging the roles of $G_{1}$ and $G_{2}$.

Let $B_{1}$ be a $\mathbb{G}_{1}-C^{*}$-algebra. Let us denote by $B_{2}:=\operatorname{Ind}_{\mathrm{G}_{1}}^{\mathrm{G}_{2}}\left(B_{1}\right)$ the induced $\mathbb{G}_{2}-C^{*}$-algebra. Let $\delta_{B_{j}}^{k}: B_{j} \rightarrow \mathcal{M}\left(B_{k} \otimes S_{k j}\right)$ for $j, k=1,2$ be the ${ }^{*}$-homomorphisms defined in 5.2.7.

6.3.16 Notations. - Let $\mathscr{E}_{1}$ be a $\mathbb{G}_{1}$-equivariant Hilbert $B_{1}$-module. Let us denote by $\mathscr{F}_{2}=\operatorname{Ind}_{\mathrm{G}_{1}}^{\mathrm{G}_{2}}\left(\mathscr{F}_{1}\right)$ the induced $\mathbb{G}_{2}$-equivariant Hilbert $B_{2}$-module. We have four linear maps

$$
\delta_{\mathscr{F}_{j}}^{k}: \mathscr{F}_{j} \rightarrow \mathcal{L}\left(B_{k} \otimes S_{k j}, \mathscr{F}_{k} \otimes S_{k j}\right), \quad \text { for } j, k=1,2,
$$

defined as follows:

- $\delta_{\mathscr{F}_{1}}^{1}:=\delta_{\mathscr{F}_{1}}$ and $\delta_{\mathscr{F}_{2}}^{2}:=\delta_{\mathscr{F}_{2}}$;

- $\delta_{\mathscr{F}_{1}}^{2}: \mathscr{F}_{1} \rightarrow \mathcal{L}\left(B_{2} \otimes S_{21}, \mathscr{F}_{2} \otimes S_{21}\right)$ is the unique linear map such that

$$
\left(\delta_{\mathscr{F}_{1}}^{2}(\xi) x\right) b=\delta_{\mathscr{F}_{1}}^{(2)}(\xi)(x b)
$$

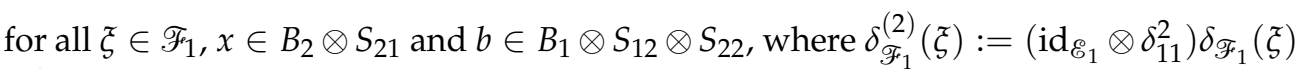
(cf. 6.3.14); 
- $\delta_{\mathscr{F}_{2}}^{1}: \mathscr{F}_{2} \rightarrow \mathcal{L}\left(B_{1} \otimes S_{12}, \mathscr{F}_{1} \otimes S_{12}\right)$ is the unique linear map such that for all $\xi \in \mathscr{F}_{2}$, $x \in \operatorname{Ind}_{\mathrm{G}_{2}}^{\mathrm{G}_{1}}\left(B_{2}\right) \otimes S_{12}$ and $y \in B_{2} \otimes S_{21} \otimes S_{12}$, we have

$$
\left[\left(\Pi_{1} \otimes \operatorname{id}_{S_{12}}\right)\left(\delta_{\mathscr{F}_{2}}^{1}(\xi)\right) x\right] y=\delta_{\mathscr{F}_{2}}^{(1)}(\xi)(x y),
$$

where $\delta_{\mathscr{F}_{2}}^{(1)}(\xi):=\left(\operatorname{id}_{\mathscr{F}_{1}} \otimes \delta_{22}^{1}\right) \delta_{\mathscr{F}_{2}}(\xi)$ and $\Pi_{1}: \mathscr{F}_{1} \rightarrow \operatorname{Ind}_{\mathrm{G}_{2}}^{\mathrm{G}_{1}}\left(\mathscr{F}_{2}\right)($ cf. 6.3.14 1 ).

6.3.17 Lemma. - For all $j, k, l=1,2$, we have the following statements:

1. $\delta_{\mathscr{F}_{j}}^{k}\left(\mathscr{F}_{j}\right) \subset \widetilde{\mathcal{M}}\left(\mathscr{F}_{k} \otimes S_{k j}\right)$;

2. $\delta_{\mathscr{F}_{j}}^{k}(\xi b)=\delta_{\mathscr{F}_{j}}^{k}(\xi) \delta_{B_{j}}^{k}(b)$ and $\left\langle\delta_{\mathscr{F}_{j}}^{k}(\xi), \delta_{\mathscr{F}_{j}}^{k}(\eta)\right\rangle=\delta_{B_{j}}^{k}(\langle\xi, \eta\rangle)$ for all $\xi, \eta \in \mathscr{F}_{j}$ and $b \in B_{j}$;

3. $\left[\delta_{\mathscr{F}_{j}}^{k}\left(\mathscr{F}_{j}\right)\left(1_{B_{k}} \otimes S_{k j}\right)\right]=\mathscr{F}_{k} \otimes S_{k j}=\left[\left(1_{\mathscr{F}_{k}} \otimes S_{k j}\right) \delta_{\mathscr{F}_{j}}^{k}\left(\mathscr{F}_{j}\right)\right]$;

4. $\delta_{\mathscr{F}_{k}}^{l} \otimes \operatorname{id}_{S_{k j}}\left(\right.$ resp. id $\left.\mathscr{F}_{l} \otimes \delta_{l j}^{k}\right)$ extends uniquely to a linear map from $\mathcal{L}\left(B_{k} \otimes S_{k j}, \mathscr{E}_{k} \otimes S_{k j}\right)$ to $\mathcal{L}\left(B_{l} \otimes S_{l k} \otimes S_{k j}, \mathscr{E}_{l} \otimes S_{l k} \otimes S_{k j}\right)$ such that

$$
\begin{aligned}
\left(\delta_{\mathscr{F}_{k}}^{l} \otimes \mathrm{id}_{S_{k j}}\right)(T)\left(\delta_{B_{k}}^{l} \otimes \mathrm{id}_{S_{k j}}\right)(x) & =\left(\delta_{\mathscr{F}_{k}}^{l} \otimes \mathrm{id}_{S_{k j}}\right)(T x) \\
\left(\text { resp. }\left(\mathrm{id}_{\mathscr{F}_{l}} \otimes \delta_{l j}^{k}\right)(T)\left(\mathrm{id}_{B_{l}} \otimes \delta_{l j}^{k}\right)(x)\right. & \left.\left.=\mathrm{id}_{\mathscr{F}_{l}} \otimes \delta_{l j}^{k}\right)(T x)\right)
\end{aligned}
$$

for all $T \in \mathcal{L}\left(B_{k} \otimes S_{k j}, \mathscr{E}_{k} \otimes S_{k j}\right)$ and $x \in B_{k} \otimes S_{k j} ;$

5. $\left(\delta_{\mathscr{F}_{k}}^{l} \otimes \mathrm{id}_{S_{k j}}\right) \delta_{\mathscr{F}_{j}}^{k}=\left(\mathrm{id}_{\mathscr{F}_{l}} \otimes \delta_{l j}^{k}\right) \delta_{\mathscr{F}_{j}}^{l}$.

Proof. Let $C:=\operatorname{Ind}_{\mathbb{G}_{2}}^{G_{1}}\left(B_{2}\right), D:=\operatorname{Ind}_{\mathbb{G}_{1}}^{\mathbb{G}_{2}}(C), \mathscr{K}=\operatorname{Ind}_{\mathbb{G}_{2}}^{\mathrm{G}_{1}}\left(\mathscr{F}_{2}\right)$ and $\mathscr{L}=\operatorname{Ind}_{\mathrm{G}_{1}}^{\mathrm{G}_{2}}(\mathscr{K})$. There exists a unique $\mathbb{G}_{2}$-equivariant unitary equivalence $\Pi_{2}: \mathscr{F}_{2} \rightarrow \mathscr{L}$ 6.3.14 1 , after exchanging the roles of $\mathbb{G}_{1}$ and $\mathbb{G}_{2}$ ) over the $\mathbb{G}_{2}$-equivariant ${ }^{*}$-isomorphism $\pi_{2}: B_{2} \rightarrow D$.

1. This statement will follow straightforwardly from the third one.

2. This statement has already been proved for $(j, k)=(1,1)$ (by definition), $(j, k)=(2,2)$ (cf. 6.3.12 (i)) and for $(j, k)=(1,2)$ (cf. 6.3.14). Moreover, the case $(j, k)=(2,1)$ follows from the formulas $\delta_{\mathscr{F}_{2}}^{1}=\left(\Pi_{1}^{-1} \otimes \mathrm{id}_{S_{12}}\right) \Pi_{2}$ and $\delta_{B_{2}}^{1}=\left(\pi_{1}^{-1} \otimes \mathrm{id}_{S_{12}}\right) \pi_{2}$.

3. This statement is true by assumption for $(j, k)=(1,1)$, for $(j, k)=(2,2)$ (cf. 6.3.12 (i)) and for $(j, k)=(1,2)$ (cf. 6.3.14 2 (ii)). By 6.3.2 and 6.3.14 we have $\left[\mathscr{L}\left(C \otimes S_{12}\right)\right]=\mathscr{K} \otimes S_{12}$, $\mathscr{L}=\Pi_{2}\left(\mathscr{F}_{2}\right)$ and $\mathscr{K}=\Pi_{1}\left(\mathscr{F}_{1}\right)$. Therefore, we have

$$
\begin{aligned}
{\left[\delta_{\mathscr{F}_{2}}^{1}\left(\mathscr{F}_{2}\right)\left(B_{1} \otimes S_{12}\right)\right] } & =\left[\left(\Pi_{1}^{-1} \otimes \mathrm{id}_{S_{12}}\right)(\mathscr{L})\left(\pi_{1}^{-1} \otimes \mathrm{id}_{S_{12}}\right)\left(D \otimes S_{12}\right)\right] \\
& =\left[\left(\Pi_{1}^{-1} \otimes \mathrm{id}_{S_{12}}\right)\left(\mathscr{L}\left(D \otimes S_{12}\right)\right)\right] \\
& =\mathscr{F}_{1} \otimes S_{12} .
\end{aligned}
$$

It then follows from the second statement and the fact that $\left[\delta_{B_{2}}^{1}\left(B_{2}\right)\left(1_{B_{1}} \otimes S_{12}\right)\right]=B_{1} \otimes S_{12}$ that $\left[\delta_{\mathscr{F}_{2}}^{1}\left(\mathscr{F}_{2}\right)\left(1_{B_{1}} \otimes S_{12}\right)\right]=\mathscr{F}_{1} \otimes S_{12}$, which is statement 3 for $(j, k)=(2,1)$.

4. Let $j, k, l=1,2$. The uniqueness of the extensions is obvious by the non-degeneracy of $\delta_{l j}^{k}$ and $\delta_{B_{k}}^{l}$. The linear map id $\mathscr{F}_{l} \otimes \delta_{l j}^{k}: \mathcal{L}\left(B_{k} \otimes S_{k j}, \mathscr{E}_{k} \otimes S_{k j}\right) \rightarrow \mathcal{L}\left(B_{l} \otimes S_{l k} \otimes S_{k j}, \mathscr{E}_{l} \otimes S_{l k} \otimes S_{k j}\right)$ is defined by

$$
\left(\operatorname{id}_{\mathscr{F}_{l}} \otimes \delta_{l j}^{k}\right)(T):=T \otimes_{\mathrm{id}_{B_{l}} \otimes \delta_{l j}^{k}} 1, \quad \text { for all } T \in \mathcal{L}\left(B_{k} \otimes S_{k j}, \mathscr{E}_{k} \otimes S_{k j}\right),
$$

where we use the identifications $(6.13)$ and $(6.17)$. As in 2.4 (a) [3], there exists a unique unitary $\mathcal{Y}_{k}^{l} \in \mathcal{L}\left(\mathscr{F}_{k} \otimes_{\delta_{B_{k}}^{l}}\left(B_{l} \otimes S_{l k}\right), \mathscr{F}_{l} \otimes S_{l k}\right)$ such that

$$
\mathscr{Y}_{k}^{l}\left(\xi \otimes_{\delta_{B_{k}}^{l}} x\right)=\delta_{\mathscr{F}_{k}}^{l}(\xi) x, \quad \text { for all } \xi \in \mathscr{F}_{k} \text { and } x \in B_{l} \otimes S_{l k} .
$$

The linear extension $\delta_{\mathscr{F}_{k}}^{l} \otimes \operatorname{id}_{S_{k j}}: \mathcal{L}\left(B_{k} \otimes S_{k j}, \mathscr{E}_{k} \otimes S_{k j}\right) \rightarrow \mathcal{L}\left(B_{l} \otimes S_{l k} \otimes S_{k j}, \mathscr{E}_{l} \otimes S_{l k} \otimes S_{k j}\right)$ is defined by $\left(\delta_{\mathscr{F}_{k}}^{l} \otimes \operatorname{id}_{S_{k j}}\right)(T):=\left(\mathcal{Y}_{k}^{l} \otimes_{C} 1\right)\left(T \otimes_{\delta_{B_{k}}^{l} \otimes \operatorname{id}_{S_{k j}}} 1\right)$ for all $T \in \mathcal{L}\left(B_{k} \otimes S_{k j}, \mathscr{E}_{k} \otimes S_{k j}\right)$, up to the identifications 6.12 and 6.16 .

5. The formula $\left(\delta_{\mathscr{F}_{k}}^{l} \otimes \mathrm{id}_{S_{k j}}\right) \delta_{\mathscr{F}_{j}}^{k}=\left(\operatorname{id}_{\mathscr{F}_{l}} \otimes \delta_{l j}^{k}\right) \delta_{\mathscr{F}_{j}}^{l}$ is derived from 6.3.12 after long but straightforward computations. 
Let us consider the $C^{*}$-algebra $B:=B_{1} \oplus B_{2}$ endowed with the continuous action $\left(\beta_{B}, \delta_{B}\right)$ (cf. 5.2.9).

6.3.18 Proposition. - Let $\mathscr{F}_{1}$ be a $\mathrm{G}_{1}$-equivariant Hilbert $B_{1}$-module. Let $\mathscr{F}_{2}:=\operatorname{Ind}_{\mathrm{G}_{1}}\left(\mathscr{F}_{1}\right)$ be the induced $\mathbb{G}_{2}$-equivariant Hilbert $B_{2}$-module. Consider the Hilbert B-module $\mathscr{F}_{F}:=\mathscr{F}_{1} \oplus \mathscr{F}_{2}$. Denote by $\Pi_{j}^{k}: \mathcal{L}\left(B_{k} \otimes S_{k j}, \mathscr{F}_{k} \otimes S_{k j}\right) \rightarrow \mathcal{L}(B \otimes S, \mathscr{F} \otimes S)$ the linear extension of the canonical injection $\mathscr{F}_{k} \otimes S_{k j} \rightarrow \mathscr{F} \otimes S$. Let us consider the linear maps $\delta_{\mathscr{F}}: \mathscr{F} \rightarrow \mathcal{L}(B \otimes S, \mathscr{F} \otimes S)$ and $\beta_{\mathscr{F}}: \mathbb{C}^{2} \rightarrow \mathcal{L}(\mathscr{F})$ defined by:

$$
\delta_{\mathscr{F}}(\xi):=\sum_{k, j=1,2} \Pi_{j}^{k} \circ \delta_{\mathscr{F}_{j}}^{k}\left(\xi_{j}\right), \quad \xi=\left(\xi_{1}, \xi_{2}\right) \in \mathscr{F} ; \quad \beta_{\mathscr{F}}(\lambda, \mu):=\left(\begin{array}{ll}
\lambda & 0 \\
0 & \mu
\end{array}\right), \quad(\lambda, \mu) \in \mathbb{C}^{2} .
$$

Then, the triple $\left(\mathscr{F}, \beta_{\mathscr{F}}, \delta_{\mathscr{F}}\right)$ is a $\mathcal{G}$-equivariant Hilbert B-module.

Proof. Let us consider $J_{1}:=\mathcal{K}\left(\mathscr{F}_{1} \oplus B_{1}\right)$ (resp. $\left.\mathcal{K}\left(\mathscr{F}_{2} \oplus B_{2}\right)\right)$ the linking $\mathrm{G}_{1}$ - $\mathrm{C}^{*}$-algebra (resp. linking $\mathbb{G}_{2}-C^{*}$-algebra) associated with $\mathscr{F}_{1}$ (resp. $\left.\mathscr{F}_{2}\right)$. Let $J_{2}:=\operatorname{Ind}_{\mathrm{G}_{1}}^{\mathbb{G}_{2}}\left(J_{1}\right)$ be the induced $\mathbb{G}_{2}-C^{*}$-algebra. Let us consider $J:=J_{1} \oplus J_{2}$ endowed with the continuous action $\left(\beta_{J}, \delta_{J}\right)$ of $\mathcal{G}$ (see above). We denote $L:=\mathcal{K}(\mathscr{F} \oplus B)$ the linking $C^{*}$-algebra associated with $\mathscr{F}$ and we identify $L=J_{1} \oplus \mathcal{K}\left(\mathscr{F}_{2} \oplus B_{2}\right)$. We have an isomorphism of linking $C^{*}$-algebras $f:=\operatorname{id}_{J_{1}} \oplus \tau: L \rightarrow J$ 6.3.12 (ii)). Let $\left(\beta_{L}, \delta_{L}\right)$ be the continuous action of $\mathcal{G}$ on $L$ obtained by transport of structure, i.e.:

$$
\delta_{L}(x):=\left(f^{-1} \otimes \operatorname{id}_{S}\right) \delta_{J}(f(x)), \quad x \in L ; \quad \beta_{L}(n):=f^{-1}\left(\beta_{J}(n)\right), \quad n \in \mathbb{C}^{2} .
$$

By straightforward computations, we show that $\left(\beta_{L}, \delta_{L}\right)$ is compatible with $\left(\beta_{B}, \delta_{B}\right)$ (cf. 6.1.8) and we prove that $\delta_{L}\left(\iota_{\mathscr{F}}(\xi)\right)=\iota_{\mathscr{F} \otimes S}\left(\delta_{\mathscr{F}}(\xi)\right)$, for all $\xi \in \mathscr{F}$. Therefore, the result follows from 6.1.11 a) and 6.1.21

6.3.19 Proposition. - Let $\left(\mathscr{E}, \beta_{\mathscr{E}}, \delta_{\mathscr{E}}\right)$ be a $\mathcal{G}$-equivariant Hilbert A-module. In the following, we use the notations of 6.2 .5 Let $j, k=1,2$ with $j \neq k$. Let

$$
\widetilde{A}_{j}:=\operatorname{Ind}_{\mathbb{G}_{k}}^{\mathbb{G}_{j}}\left(A_{k}, \delta_{A_{k}}^{k}\right) \quad \text { and } \quad \widetilde{\mathscr{C}}_{j}:=\operatorname{Ind}_{\mathbb{G}_{k}}^{\mathbb{G}_{j}}\left(\mathscr{E}_{k}, \delta_{\mathscr{E}_{k}}^{k}\right) \text {. }
$$

If $\xi \in \mathscr{E}_{j}$, then we have $\delta_{\mathscr{E}_{j}}^{k}(\xi) \in \widetilde{\mathscr{E}}_{j} \subset \widetilde{\mathcal{M}}\left(\mathscr{E}_{k} \otimes S_{k j}\right)$ and the map $\widetilde{\Pi}_{j}: \mathscr{E}_{j} \rightarrow \widetilde{\mathscr{E}}_{j} ; \xi \mapsto \delta_{\mathscr{E}_{j}}^{k}(\xi)$ is a $\mathbb{G}_{j}$-equivariant unitary equivalence over $\widetilde{\pi}_{j}: A_{j} \rightarrow \widetilde{A}_{j}(c f .5 .2 .8$. .

Proof. We have $\mathscr{E}_{j}=\left[\left(\operatorname{id}_{\mathscr{E}_{j}} \otimes \omega\right) \delta_{\mathscr{E}_{k}}^{j}(\xi) ; \omega \in \mathcal{B}\left(\mathscr{H}_{j k}\right)_{*}, \xi \in \mathscr{E}_{k}\right]$ (cf. 6.2.5 (iv)) and for all $\xi \in \mathscr{E}_{j}$ and $\omega \in \mathcal{B}\left(\mathscr{H}_{j k}\right)_{*}$ we have

$$
\delta_{\mathscr{E}_{j}}^{k}\left(\mathrm{id}_{\mathscr{\delta}_{j}} \otimes \omega\right) \delta_{\mathscr{E}_{k}}^{j}(\xi)=\left(\mathrm{id}_{\mathscr{E}_{k} \otimes S_{k j}} \otimes \omega\right)\left(\delta_{\mathscr{E}_{j}}^{k} \otimes \mathrm{id}_{S_{j k}}\right) \delta_{\mathscr{E}_{k}}^{j}(\xi)=\left(\mathrm{id}_{\mathscr{E}_{k} \otimes S_{k j}} \otimes \omega\right) \delta_{\mathscr{E}_{j}}^{(k)}(\xi)
$$

(cf. 6.2.5 (v)), where $\delta_{\mathscr{E}_{j}}^{(k)}(\xi):=\left(\mathrm{id}_{\mathscr{\delta}_{j}} \otimes \delta_{j j}^{k}\right) \delta_{\mathscr{E}_{j}}^{j}(\xi)$. As a consequence, statement $\mathrm{I}$ is proved as well as the surjectivity of $\widetilde{\Pi}_{j}$. The fact that $\widetilde{\Pi}_{j}$ is a $\mathbb{G}_{j}$-equivariant $\widetilde{\pi}_{j}$-compatible unitary operator is just a restatement of 6.2.5 (iii) and $\left(\mathrm{id}_{\mathscr{E}_{k}} \otimes \delta_{k j}^{j}\right) \delta_{\mathscr{E}_{j}}^{k}=\left(\delta_{\mathscr{E}_{j}}^{k} \otimes \mathrm{id}_{S_{j j}}\right) \delta_{\mathscr{E}_{j}}^{j}$ 6.2.5
(v)).

6.3.20 THEOREM. - Let $\mathcal{G}_{\mathrm{G}_{1}, \mathrm{G}_{2}}$ be a colinking measured quantum groupoid between two regular monoidally equivalent locally compact quantum groups $\mathrm{G}_{1}$ and $\mathrm{G}_{2}$. Let $j=1,2$. The map $\left(\mathscr{E}, \beta_{\mathscr{E}}, \delta_{\mathscr{E}}\right) \mapsto\left(\mathscr{E}_{j}, \delta_{\mathscr{E}_{j}}^{j}\right)$ is a one-to-one correspondence up to unitary equivalence (cf. 6.2.5 and 6.2.6 1). The inverse map, up to unitary equivalence, is $\left(\mathscr{F}_{j}, \delta_{\mathscr{F}_{j}}\right) \mapsto\left(\mathscr{F}, \beta_{\mathscr{F}}, \delta_{\mathscr{F}}\right)(c f .6 .3 .18] 6.3 .13$ and 6.2.62).

Proof. Let $A$ be a $\mathcal{G}$-C*-algebra and $\mathscr{E}$ a $\mathcal{G}$-equivariant Hilbert $A$-module. Let us use all the notations introduced in 86.2 . Let us denote:

$$
\begin{aligned}
& \left(B_{1}, \delta_{B_{1}}\right):=\left(A_{1}, \delta_{A_{1}}^{1}\right),\left(B_{2}, \delta_{B_{2}}\right):=\operatorname{Ind}_{\mathbb{G}_{1}}^{\mathbb{G}_{2}}\left(B_{1}, \delta_{B_{1}}\right) ; \\
& \left(\mathscr{F}_{1}, \delta_{\mathscr{F}_{1}}\right):=\left(\mathscr{E}_{1}, \delta_{\mathscr{E}_{1}}^{1}\right),\left(\mathscr{F}_{2}, \delta_{\mathscr{F}_{2}}\right):=\operatorname{Ind}_{\mathbb{G}_{1}}^{G_{2}}\left(\mathscr{F}_{1}, \delta_{\mathscr{F}_{1}}\right) .
\end{aligned}
$$


Let us endow the $C^{*}$-algebra $B:=B_{1} \oplus B_{2}$ with the continuous action $\left(\beta_{B}, \delta_{B}\right)$ of $\mathcal{G}$ and $\mathscr{F}_{F}:=\mathscr{F}_{1} \oplus \mathscr{F}_{2}$ with the structure of $\mathcal{G}$-equivariant Hilbert $B$-module $\left(\beta_{\mathscr{F}}, \delta_{\mathscr{F}}\right)$ (cf. 5.2.9. 6.3.18). Let $\psi_{A}: A \rightarrow B$ the canonical $\mathcal{G}$-equivariant ${ }^{*}$-isomorphism defined for all $a \in A$ by $\psi_{A}(a):=\left(q_{A, 1} a, \widetilde{\pi}_{2}\left(q_{A, 2} a\right)\right)$ (cf. $\left.4.10[2]\right)$. Then, we consider the map $\Psi: \mathscr{E} \rightarrow \mathscr{F}$ given by

$$
\Psi(\xi):=\left(q_{\mathscr{E}, 1} \xi, \widetilde{\Pi}_{2}\left(q_{\mathscr{E}, 2} \xi\right)\right), \text { for all } \xi \in \mathscr{E} .
$$

It is clear from 6.3.19 that $\Psi$ is a $\psi_{A}$-compatible unitary operator. Let us consider the $\mathcal{G}$ - $C^{*}$-algebras $K:=\mathcal{K}(\mathscr{E} \oplus A)$ and $L:=\mathcal{K}(\mathscr{F} \oplus B)$. Let $f: K \rightarrow L$ be the associated isomorphism of linking $C^{*}$-algebras (cf. 8.3.5). In virtue of 6.1.18, it only remains to prove that $f$ is $\mathcal{G}$-equivariant. We also consider the $G_{1}-C^{*}$-algebra $J_{1}:=\mathcal{K}\left(\mathscr{F}_{1} \oplus B_{1}\right)$ and the induced $\mathbb{G}_{2}-C^{*}$-algebra $J_{2}:=\operatorname{Ind}_{\mathbb{G}_{1}}^{\mathbb{G}_{2}}\left(J_{1}\right)$. We recall that we have a canonical isomorphism $\tau: \mathcal{K}\left(\mathscr{F}_{2} \oplus B_{2}\right) \rightarrow J_{2}$ (cf. 6.3.12 (ii)). Let us endow the $C^{*}$-algebra $J:=J_{1} \oplus J_{2}$ with the continuous action $\left(\beta_{J}, \delta_{J}\right)$ of $\mathcal{G}$. Therefore, it amounts to proving that the ${ }^{*}$-isomorphism $\left(\operatorname{id}_{J_{1}} \oplus \tau\right) f: K \rightarrow J$ is $\mathcal{G}$-equivariant (we identify $L=J_{1} \oplus \mathcal{K}\left(\mathscr{F}_{2} \oplus B_{2}\right)$ ). We apply the notations of $\$ 6.2$ to the $\mathcal{G}$-C ${ }^{*}$-algebra $K$ and identify $K_{j}:=q_{K, j} K=\mathcal{K}\left(\mathscr{E}_{j} \oplus A_{j}\right)$ for $j=1$, 2 . Let us consider as above (by exchanging the roles of $A$ and $K$ ) the $\mathcal{G}$-equivariant ${ }^{*}$-isomorphism $\psi_{K}: K \rightarrow J$. By evaluating on elements of the form $\iota_{\mathscr{E}}(\xi)$ for $\xi \in \mathscr{E}$ and $\iota_{A}(a)$ for $a \in A$, it is staightforward to see that $\left(\operatorname{id}_{J_{1}} \oplus \tau\right) f=\psi_{K}$.

\section{TAKESAKI-TAKAI DUALITY AND EQUIVARIANT MORITA EQUIVALENCE}

In this section, we fix a measured quantum groupoid $\mathcal{G}=\left(N, M, \alpha, \beta, \Delta, T, T^{\prime}, \epsilon\right)$ on the finite-dimensional basis $N=\bigoplus_{1 \leqslant l \leqslant k} \mathrm{M}_{n_{l}}(\mathbb{C})$ and we use all the notations introduced in $\S \S$ 3.1 and 3.2 We will use the notations and results of $\$ \$ 5.1$. 5.3 and 6.1

EQUiVARIANT HILBERT BIMOdUles AND MORITA EQUiVAlenCe. In this paragraph, we introduce the notion of equivariant representation of a $\mathcal{G}$ - $C^{*}$-algebra on a Hilbert module acted upon by $\mathcal{G}$. We then introduce the notion of equivariant Morita equivalence.

7.1 Notation. - Let $A$ and $B$ be $C^{*}$-algebras. Let $\mathscr{E}$ be a Hilbert $B$-module. If $\gamma: A \rightarrow \mathcal{L}(\mathscr{E})$ is a *-homomorphism then, up to the identification $\mathcal{M}(\mathcal{K}(\mathscr{E}) \otimes S)=\mathcal{L}(\mathscr{E} \otimes S)$, we can extend $\gamma \otimes \mathrm{id}_{S}$ to a ${ }^{*}$-homomorphism $\gamma \otimes \operatorname{id}_{S}: \widetilde{\mathcal{M}}(A \otimes S) \rightarrow \mathcal{L}(\mathscr{E} \otimes S)$ (cf. $\& \mathbb{1}$ ).

As in $2.9[3]$, we have:

7.2 Definition. - Let $A$ and $B$ be two $\mathcal{G}$ - $C^{*}$-algebras, $\mathscr{E}$ a Hilbert $B$-module, $\left(\beta_{\mathscr{E}}, \delta_{\mathscr{E}}\right)$ an action of $\mathcal{G}$ on $\mathscr{E}$ and $\gamma: A \rightarrow \mathcal{L}(\mathscr{E})$ a ${ }^{*}$-representation. We say that $\gamma$ is $\mathcal{G}$-equivariant if we have:

1. $\delta_{\mathscr{E}}(\gamma(a) \xi)=\left(\gamma \otimes \operatorname{id}_{\mathcal{S}}\right)\left(\delta_{A}(a)\right) \circ \delta_{\mathscr{E}}(\xi)$, for all $a \in A$ and $\xi \in \mathscr{E}$;

2. $\beta_{\mathscr{E}}\left(n^{\mathrm{o}}\right) \circ \gamma(a)=\gamma\left(\beta_{A}\left(n^{\mathrm{o}}\right) a\right)$, for all $n \in N$ and $a \in A$.

7.3 Remarks. - 1. Provided that the second condition in the above definition is verified, the first condition is equivalent to:

$$
\mathcal{V}\left(\gamma(a) \otimes_{\delta_{B}} 1\right)^{*} \mathcal{V}^{*}=\left(\gamma \otimes \operatorname{id}_{S}\right) \delta_{A}(a), \text { for all } a \in A,
$$

where $\mathcal{V} \in \mathcal{L}\left(\mathscr{E} \otimes_{\delta_{B}}(B \otimes S), \mathscr{E} \otimes S\right)$ denotes the isometry defined in 6.1.5 a). Indeed, we can interpret it as follows: $\mathcal{V}\left(\gamma(a) \otimes_{\delta_{B}} 1\right)=\left(\gamma \otimes \operatorname{id}_{S}\right)\left(\delta_{A}(a)\right) \mathcal{V}$, for all $a \in A$. Moreover, for all $a \in A$ we have

$$
\begin{aligned}
\left(\gamma \otimes \operatorname{id}_{S}\right)\left(\delta_{A}(a)\right)^{\mathscr{V} \mathcal{V}^{*}} & =\left(\gamma \otimes \operatorname{id}_{S}\right)\left(\delta_{A}(a)\right) q_{\beta_{\S} \alpha} \\
& =\left(\gamma \otimes \operatorname{id}_{S}\right)\left(\delta_{A}(a) q_{\beta_{A} \alpha}\right) \\
& =\left(\gamma \otimes \operatorname{id}_{S}\right) \delta_{A}(a) .
\end{aligned}
$$

Hence, $\left(\mathcal{V}\left(\gamma(a) \otimes_{\delta_{B}} 1\right)=\left(\gamma \otimes \operatorname{id}_{S}\right)\left(\delta_{A}(a)\right) \mathcal{V} \Leftrightarrow \mathcal{V}\left(\gamma(a) \otimes_{\delta_{B}} 1\right) \mathscr{V}^{*}=\left(\gamma \otimes \operatorname{id}_{S}\right) \delta_{A}(a)\right)$, for all $a \in A$. 
2. We recall that the action $\delta_{\mathcal{K}(\mathscr{E})}$ of $\mathcal{G}$ on $\mathcal{K}(\mathscr{E})$ is defined by $\delta_{\mathcal{K}(\mathscr{E})}(k):=\mathscr{V}\left(k \otimes_{\delta_{B}} 1\right)^{\mathcal{V}}{ }^{*}$ for all $k \in \mathcal{K}(\mathscr{E})$. Hence, $\sqrt{7 \cdot 1})$ can be restated as follows: $\delta_{\mathcal{K}(\mathscr{E})}(\gamma(a))=\left(\gamma \otimes \mathrm{id}_{S}\right) \delta_{A}(a)$ for all $a \in A$. In particular, if $\gamma$ is non-degenerate, then Definition 7.2 simply means that the ${ }^{*}$-homomorphism $\gamma: A \rightarrow \mathcal{M}(\mathcal{K}(\mathscr{E}))$ is $\mathcal{G}$-equivariant (cf. 5.1.10.

3. If $\gamma: A \rightarrow \mathcal{L}(\mathscr{E})$ is a non-degenerate ${ }^{*}$-representation such that

$$
\delta_{\mathscr{E}}(\gamma(a) \xi)=\left(\gamma \otimes \operatorname{id}_{S}\right)\left(\delta_{A}(a)\right) \circ \delta_{\mathscr{E}}(\xi) \text {, for all } a \in A \text { and } \xi \in \mathscr{E} \text {, }
$$

then we have $\beta_{\mathscr{E}}\left(n^{\mathrm{o}}\right) \circ \gamma(a)=\gamma\left(\beta_{A}\left(n^{\mathrm{o}}\right) a\right)$ for all $n \in N$ and $a \in A$. Indeed, this will be inferred from 5.1.11 and the previous remark.

7.4 Definition. - (cf. $\S 6$ [22]) Let $A$ and $B$ be two $C^{*}$-algebras. An imprimitivity $A-B$ bimodule is an $A$-B-bimodule $\mathscr{E}$, which is a full left Hilbert $A$-module for an $A$-valued inner product ${ }_{A}\langle\cdot, \cdot\rangle$ and a full right Hilbert $B$-module for a $B$-valued inner product $\langle\cdot, \cdot\rangle_{B}$ such that $A\langle\xi, \eta\rangle \zeta=\xi\langle\eta, \zeta\rangle_{B}$ for all $\xi, \eta, \zeta \in \mathscr{E}$.

7.5 Remarks. - Let $A$ and $B$ be two $C^{*}$-algebras and $\mathscr{E}$ an imprimitivity $A$ - $B$-bimodule. We recall that the norms defined by the inner products $A\langle\cdot, \cdot\rangle$ on $\mathscr{E}^{\mathscr{E}}$ and $\langle\cdot, \cdot\rangle_{B}$ on $\mathscr{E}_{B}$ coincide. We also recall that the left (resp. right) action of $A$ (resp. $B$ ) on $\mathscr{E}$ defines a non-degenerate *-homomorphism $\gamma: A \rightarrow \mathcal{L}\left(\mathscr{E}_{B}\right)$ (resp. $\left.\rho: B \rightarrow \mathcal{L}\left({ }_{A} \mathscr{E}\right)\right)$.

7.6 Definition. - Let $A$ and $B$ be two $\mathcal{G}$ - $C^{*}$-algebras. A $\mathcal{G}$-equivariant imprimitivity $A$ - $B$-bimodule is an imprimitivity $A$-B-bimodule $\mathscr{E}$ endowed with a continuous action of $\mathcal{G}$ on $\mathscr{E}_{B}$ such that the left action $\gamma: A \rightarrow \mathcal{L}\left(\mathscr{E}_{B}\right)$ is $\mathcal{G}$-equivariant.

7.7 Examples. - Let $A$ and $B$ be two $\mathcal{G}-C^{*}$-algebras.

(i) $B$ is a $\mathcal{G}$-equivariant imprimitivity $B$ - $B$-bimodule for the inner products given by ${ }_{B}\langle x, y\rangle:=x y^{*}$ and $\langle x, y\rangle_{B}:=x^{*} y$ for all $x, y \in B$.

(ii) Let $\mathscr{E}$ be a $\mathcal{G}$-equivariant Hilbert $B$-module. If $\mathscr{E}$ is full, then $\mathscr{E}$ is a $\mathcal{G}$-equivariant imprimitivity $\mathcal{K}(\mathscr{E})$ - $B$-bimodule for the natural left action and the inner product given by $\mathcal{K}(\mathscr{E})\langle\xi, \eta\rangle:=\theta_{\xi, \eta}$ for all $\xi, \eta \in \mathscr{E}$. Conversely, if $\mathscr{E}$ is a $\mathcal{G}$-equivariant imprimitivity $A$-B-bimodule, then the the left action $\gamma: A \rightarrow \mathcal{L}\left(\mathscr{E}_{B}\right)$ induces an isomorphism of $\mathcal{G}$-C*algebras $A \simeq \mathcal{K}\left(\mathscr{E}_{B}\right)$.

(iii) Let $\left(J, \beta_{J}, \delta_{J}, e_{1}, e_{2}\right)$ be a linking $\mathcal{G}-C^{*}$-algebra (cf.6.1.22. Let $A:=e_{1} J e_{1}$ and $B:=e_{2} J e_{2}$ be the corner $C^{*}$-algebras endowed with the continuous actions of $\mathcal{G}$ induced by $\left(\beta_{J}, \delta_{J}\right)$. Let us endow $\mathscr{E}:=e_{1} J e_{2}$ with its structure of $\mathcal{G}$-equivariant Hilbert $B$-module (cf. 6.1.23. Then, $\mathscr{E}$ is a $\mathcal{G}$-equivariant imprimitivity $A$ - $B$-module whose actions and inner products are defined as in (i).

(iv) If $\mathscr{E}$ is a $\mathcal{G}$-equivariant imprimitivity $A$ - $B$-bimodule, then $\mathscr{E}^{*}$ turns into a $\mathcal{G}$-equivariant imprimitivity $B$ - $A$-bimodule for the actions and inner products given by the following formulas: $b \xi^{*} a:=\left(a^{*} \xi b^{*}\right)^{*}$, for $\xi^{*} \in \mathscr{E}^{*}, a \in A$ and $b \in B ;{ }_{B}\left\langle\xi^{*}, \eta^{*}\right\rangle:=\langle\xi, \eta\rangle_{B}$ and $\left\langle\xi^{*}, \eta^{*}\right\rangle_{A}:={ }_{A}\langle\xi, \eta\rangle$, for $\xi^{*}, \eta^{*} \in \mathscr{E}^{*}$.

7.8 Proposition. - Let $A$ and $B$ be $\mathcal{G}$ - $C^{*}$-algebras. The following statements are equivalent:

(i) there exists a $\mathcal{G}$-equivariant imprimitivity $A$-B-bimodule;

(ii) there exists a full $\mathcal{G}$-equivariant Hilbert B-module $\mathscr{E}$ such that we have an isomorphism $A \simeq \mathcal{K}(\mathscr{E})$ of $\mathcal{G}-C^{*}$-algebras;

(iii) there exists a linking $\mathcal{G}$ - $C^{*}$-algebra $\left(J, \beta_{J}, \delta_{J}, e_{1}, e_{2}\right)$ such that we have $\mathcal{G}$-equivariant *isomorphisms $A \simeq e_{1} J e_{1}$ and $B \simeq e_{2} J e_{2}$.

Proof. This is a straightforward consequence of 7.7 (ii), (iii), 6.1.11 b) and 6.1.23

Now, we investigate the tensor product construction (cf. 2.10 [3] for the quantum group case). 
7.9 Proposition. - Let $C$ (resp. B) be a $\mathcal{G}$-C $C^{*}$-algebra. Let $\mathscr{E}_{1}\left(\right.$ resp. $\left.\mathscr{E}_{2}\right)$ be a Hilbert module over $C\left(\right.$ resp. B) endowed with an action $\left(\beta_{\mathscr{E}_{1}}, \delta_{\mathscr{E}_{1}}\right)\left(\right.$ resp. $\left.\left(\beta_{\mathscr{E}_{2}}, \delta_{\mathscr{E}_{2}}\right)\right)$ of $\mathcal{G}$. Let $\gamma_{2}: C \rightarrow \mathcal{L}\left(\mathscr{E}_{2}\right)$ be a $\mathcal{G}$-equivariant ${ }^{*}$-representation. Consider the Hilbert $B$-module $\mathscr{E}:=\mathscr{E}_{1} \otimes_{\gamma_{2}} \mathscr{E}_{2}$. Denote by

$$
\Delta\left(\xi_{1}, \xi_{2}\right):=\left(\delta_{\mathscr{E}_{1}}\left(\xi_{1}\right) \otimes_{\tilde{\gamma}_{2} \otimes \mathrm{id}_{S}} 1\right) \circ \delta_{\mathscr{E}_{2}}\left(\xi_{2}\right), \text { for } \xi_{1} \in \mathscr{E}_{1} \text { and } \xi_{2} \in \mathscr{E}_{2} \text {. }
$$

We have $\Delta\left(\xi_{1}, \xi_{2}\right) \in \widetilde{\mathcal{M}}(\mathscr{E} \otimes S)$ for all $\xi_{1} \in \mathscr{E}_{1}$ and $\xi_{2} \in \mathscr{E}_{2}$. Let $\beta_{\mathscr{E}}: N^{\mathrm{o}} \rightarrow \mathcal{L}(\mathscr{E})$ be the *-homomorphism defined by

$$
\beta_{\mathscr{E}}\left(n^{\mathrm{o}}\right):=\beta_{\mathscr{E}_{1}}\left(n^{\mathrm{o}}\right) \otimes_{\gamma_{2}} 1, \text { for all } n \in N \text {. }
$$

There exists a unique map $\delta_{\mathscr{E}}: \mathscr{E} \rightarrow \widetilde{\mathcal{M}}(\mathscr{E} \otimes S)$ defined by the formula $\delta_{\mathscr{E}}\left(\xi_{1} \otimes_{\gamma_{2}} \xi_{2}\right):=\Delta\left(\xi_{1}, \xi_{2}\right)$ for $\xi_{1} \in \mathscr{E}_{1}$ and $\xi_{2} \in \mathscr{E}_{2}$ such that the pair $\left(\beta_{\mathscr{E}}, \delta_{\mathscr{E}}\right)$ is an action of $\mathcal{G}$ on $\mathscr{E}$.

The operator $\delta_{\mathscr{E}_{1}}\left(\widetilde{\xi}_{1}\right)$ is considered here as an element of $\mathcal{L}\left(\widetilde{C} \otimes S, \mathscr{E}_{1} \otimes S\right) \supset \widetilde{\mathcal{M}}\left(\mathscr{E}_{1} \otimes S\right)$. In particular, we have $\delta_{\mathscr{E}_{1}}\left(\xi_{1}\right) \otimes_{\tilde{\gamma}_{2} \otimes \mathrm{id}_{S}} 1 \in \mathcal{L}\left(\mathscr{E}_{2} \otimes S, \mathscr{E} \otimes S\right)$ since we use the identifications:

$$
\begin{aligned}
& (\widetilde{C} \otimes S) \otimes_{\tilde{\gamma}_{2} \otimes \mathrm{id}_{S}}\left(\mathscr{E}_{2} \otimes S\right)=\mathscr{E}_{2} \otimes S, x \otimes_{\tilde{\gamma}_{2} \otimes \mathrm{id}_{S}} \eta \mapsto\left(\widetilde{\gamma}_{2} \otimes \mathrm{id}_{S}\right)(x) \eta ; \\
& \left(\mathscr{E}_{1} \otimes S\right) \otimes_{\tilde{\gamma}_{2} \otimes \mathrm{id}_{S}}\left(\mathscr{E}_{2} \otimes S\right)=\mathscr{E} \otimes S,\left(\xi_{1} \otimes S\right) \otimes_{\tilde{\gamma}_{2} \otimes \mathrm{id}_{S}}\left(\xi_{2} \otimes t\right) \mapsto\left(\xi_{1} \otimes_{\gamma_{2}} \xi_{2}\right) \otimes s t .
\end{aligned}
$$

Proof. The proof is basically the same as that of 2.10 [3]. For example, we refer the reader to it for the proof of the fact that $\Delta\left(\xi_{1}, \xi_{2}\right) \in \overline{\mathcal{M}}(\mathscr{E} \otimes S)$ for all $\xi_{1} \in \mathscr{E}_{1}$ and $\xi_{2} \in \mathscr{E}$. Let $\mathscr{V}_{1}$ and $\mathscr{V}_{2}$ be the isometries associated with $\delta_{\mathscr{E}_{1}}$ and $\delta_{\mathscr{E}_{2}}$. Since $\mathscr{V}_{2}$ intertwines the left actions $c \mapsto \gamma_{2}(c) \otimes_{\delta_{B}} 1$ and $\left(\gamma_{2} \otimes \mathrm{id}_{S}\right) \delta_{C}$ of $C$, there exists a unique isometry $\widetilde{\mathcal{V}}_{2} \in \mathcal{L}\left(\mathscr{E} \otimes_{\delta_{B}}(B \otimes S), \mathscr{E}_{1} \otimes\left(\gamma_{2} \otimes \mathrm{id}_{S}\right) \delta_{C}\left(\mathscr{E}_{2} \otimes S\right)\right)$ such that

$\widetilde{\mathscr{V}}_{2}\left(\left(\xi_{1} \otimes_{\gamma_{2}} \xi_{2}\right) \otimes_{\delta_{B}} x\right)=\xi_{1} \otimes_{\left(\gamma_{2} \otimes i d_{S}\right) \delta_{C}} \mathscr{V}_{2}\left(\xi_{2} \otimes_{\delta_{B}} x\right)$, for all $\xi_{1} \in \mathscr{E}_{1}, \xi_{2} \in \mathscr{E}_{2}$ and $x \in B \otimes S$.

Let us prove that $\widetilde{\mathscr{V}}_{2}$ is a unitary. It amounts to proving that $\widetilde{\mathscr{V}}_{2}$ is surjective. Since $\operatorname{im}\left(\mathscr{V}_{2}\right)=\operatorname{im}\left(q_{\beta_{\varepsilon_{2}} \alpha}\right)$, we have $\operatorname{im}\left(\widetilde{\mathscr{V}}_{2}\right)=\left[\xi \otimes\left(_{\left.\gamma_{2} \otimes \mathrm{id}_{S}\right) \delta_{C}} q_{\beta_{\delta_{2}} \alpha} \eta ; \xi \in \mathscr{E}_{1}, \eta \in \mathscr{E}_{2} \otimes S\right]\right.$. Let $\xi \in \mathscr{E}_{1}$ and $\eta \in \mathscr{E}_{2} \otimes S$. Write $\xi=\xi^{\prime} c$ with $\xi^{\prime} \in \mathscr{E}_{1}$ and $c \in C$. Since $\mathscr{V}_{2} \mathcal{V}_{2}^{*}=q_{\mathscr{E}_{2} \alpha}$, we have $\left(\gamma_{2} \otimes \mathrm{id}_{S}\right) \delta_{C}(c) q_{\beta_{\varepsilon_{2} \alpha}}=\left(\gamma_{2} \otimes \mathrm{id}_{S}\right) \delta_{C}(c)$ (cf. $7 \cdot 3$. Hence,

$$
\begin{aligned}
\xi \otimes_{\left(\gamma_{2} \otimes \mathrm{id}_{S}\right) \delta_{C}} q_{\beta_{\delta_{2}} \alpha} \eta & =\xi^{\prime} \otimes_{\left(\gamma_{2} \otimes \mathrm{id}_{S}\right) \delta_{C}}\left(\gamma_{2} \otimes \mathrm{id}_{S}\right) \delta_{C}(c) q_{\beta_{\delta_{2}} \alpha} \eta \\
& =\xi^{\prime} \otimes_{\left(\gamma_{2} \otimes \mathrm{id}_{S}\right) \delta_{C}}\left(\gamma_{2} \otimes \mathrm{id}_{S}\right) \delta_{C}(c) \eta \\
& =\xi \otimes_{\left(\gamma_{2} \otimes \mathrm{id}_{S}\right) \delta_{C}} \eta .
\end{aligned}
$$

Therefore we have shown that $\operatorname{im}\left(\widetilde{\mathscr{V}}_{2}\right)=\mathscr{E}_{1} \otimes\left(\gamma_{2} \otimes \mathrm{id} S\right) \delta_{C}\left(\mathscr{E}_{2} \otimes S\right)$, which proves that $\widetilde{\mathscr{V}}_{2}$ is unitary. Let us identify

$$
\begin{aligned}
\left(\mathscr{E}_{1} \otimes_{\delta_{C}}(C \otimes S)\right) \otimes_{\gamma_{2} \otimes \mathrm{id}_{S}}\left(\mathscr{E}_{2} \otimes S\right) & \rightarrow \mathscr{E}_{1} \otimes_{\left(\gamma_{2} \otimes \mathrm{id}_{S}\right) \delta_{C}}\left(\mathscr{E}_{2} \otimes S\right) \\
\left(\xi_{1} \otimes_{\delta_{C}} x\right) \otimes_{\gamma_{2} \otimes \mathrm{id}_{S}} \eta & \mapsto \xi_{1} \otimes_{\left(\gamma_{2} \otimes \mathrm{id}_{S}\right) \delta_{C}}\left(\gamma_{2} \otimes \mathrm{id}_{S}\right)(x) \eta
\end{aligned}
$$

and $\left(\mathscr{E}_{1} \otimes S\right) \otimes_{\gamma_{2} \otimes \mathrm{id}_{S}}\left(\mathscr{E}_{2} \otimes S\right)=\mathscr{E} \otimes S$ (cf. [7.3) $)$. Let

$$
\mathcal{V}:=\left(\mathscr{V}_{1} \otimes_{\gamma_{2} \otimes \mathrm{id}_{S}} 1\right) \widetilde{\mathcal{V}}_{2} \in \mathcal{L}\left(\mathscr{E} \otimes_{\delta_{B}}(B \otimes S), \mathscr{E} \otimes S\right) .
$$

It follows from the formulas $\widetilde{\mathcal{V}}_{2}^{*} \widetilde{\mathcal{V}}_{2}=1, \widetilde{\mathcal{V}}_{2} \widetilde{\mathcal{V}}_{2}^{*}=1, \mathscr{V}_{1}^{* \mathfrak{V}} \mathcal{V}_{1}=1$ and $\mathscr{V}_{1} \mathscr{V}_{1}^{*}=q_{\beta_{8_{1}} \alpha}$ that $\mathcal{V}^{*} \mathcal{V}=1$ and $\mathscr{V}^{*}=q_{\beta_{\delta_{1} \alpha}} \otimes_{\gamma_{2} \otimes \mathrm{id}_{S}} 1=q_{\beta_{\mathscr{E}} \alpha}$ (by definition of $\beta_{\mathscr{E}}$ ).

Let $n \in N$. On one hand, we have

$$
\widetilde{\mathcal{V}}_{2}\left(\beta_{\mathscr{E}}\left(n^{\mathrm{o}}\right) \otimes_{\delta_{B}} 1\right)=\left(\beta_{\mathscr{E}_{1}}\left(n^{\mathrm{o}}\right) \otimes_{\left(\gamma_{2} \otimes \mathrm{id}_{S}\right) \delta_{C}} 1\right)^{\mathscr{V}_{2}}
$$

(by definition of $\beta_{\mathscr{E}}$ and $\widetilde{\mathcal{V}}_{2}$ ). On the other, we have

$$
\left(\mathscr{V}_{1} \otimes_{\gamma_{2} \otimes \mathrm{id}_{S}} 1\right)\left(\beta_{\mathscr{E}_{1}}\left(n^{\mathrm{o}}\right) \otimes_{\left(\gamma_{2} \otimes \mathrm{id}_{S}\right) \delta_{C}} 1\right)=\left(\left(1_{\mathscr{E}_{1}} \otimes \beta\left(n^{\mathrm{o}}\right)\right) \otimes_{\gamma_{2} \otimes \mathrm{id}_{S}} 1\right)\left(\mathscr{V}_{1} \otimes_{\gamma_{2} \otimes \mathrm{id}_{S}} 1\right) .
$$


Hence, we have proved that $\mathcal{V}\left(\beta_{\mathscr{E}}\left(n^{\mathrm{o}}\right) \otimes_{\delta_{B}} 1\right)=\left(1 \otimes \beta\left(n^{\mathrm{o}}\right)\right) \mathcal{V}$ for all $n \in N$. Exactly as in the proof of $2.10[3]$, we have $\mathcal{V} T_{\xi_{1} \otimes_{\gamma_{2}} \xi_{2}}=\Delta\left(\xi_{1}, \xi_{2}\right)$ for all $\xi_{1} \in \mathscr{E}_{1}$ and $\xi_{2} \in \mathscr{E}_{2}$ (cf. 6.1.3 for the definition of $\left.T_{\xi}\right)$. In particular, $\mathcal{V} T_{\xi} \in \widetilde{\mathcal{M}}(\mathscr{E} \otimes S)$ for all $\xi \in \mathscr{E}$. It then follows from 6.1.5 b) that the pair $\left(\beta_{\mathscr{E}}, \delta_{\mathscr{E}}\right)$, where $\delta_{\mathscr{E}}: \mathscr{E} \rightarrow \widetilde{\mathcal{M}}(\mathscr{E} \otimes S)$ is defined for all $\xi \in \mathscr{E}$ by $\delta_{\mathscr{E}}(\xi):=\mathcal{V} T_{\tilde{\zeta}}$, satisfies the conditions 1,2 , and 3 of Definition 6.1.4. The coassociativity condition of $\delta_{\mathscr{E}}$ is derived from those of $\delta_{\mathscr{E}_{1}}$ and $\delta_{\mathscr{E}_{2}}$ exactly as in the proof of 2.10 [3].

7.1o Proposition. - We use all the notations and hypotheses of 7.9 If $A$ is a $\mathcal{G}$-C*-algebra and $\gamma_{1}: A \rightarrow \mathcal{L}\left(\mathscr{E}_{1}\right)$ is a $\mathcal{G}$-equivariant ${ }^{*}$-representation, then $\gamma: A \rightarrow \mathcal{L}\left(\mathscr{E}_{1} \otimes_{\gamma_{2}} \mathscr{E}_{2}\right)$ the *-representation defined by $\gamma(a):=\gamma_{1}(a) \otimes_{\gamma_{2}} 1$ for all $a \in A$ is $\mathcal{G}$-equivariant.

Proof. Through the identification 7.3 , for all $x \in A \otimes S$ the operator $\left(\gamma_{1} \otimes \mathrm{id}_{S}\right)(x) \otimes \tilde{\gamma}_{2} \otimes \mathrm{id}_{S} 1$ is identified to $\left(\gamma \otimes \mathrm{id}_{S}\right)(x)$. This identification also holds for $x \in \widetilde{\mathcal{M}}(A \otimes S)$ (by using the fact that any element of $S$ can be written as a product of two elements of $S)$. In particular, for all $a \in A$ the operator $\left(\gamma_{1} \otimes \operatorname{id}_{S}\right) \delta_{A}(a) \otimes_{\tilde{\gamma}_{2} \otimes \mathrm{id}_{S}} 1$ is identified to $\left(\gamma \otimes \operatorname{id}_{S}\right) \delta_{A}(a)$. Hence, $\delta_{\mathscr{E}}(\gamma(a) \xi)=\left(\gamma \otimes \mathrm{id}_{S}\right) \delta_{A}(a) \circ \delta_{\mathscr{E}}(\xi)$ for all $\xi \in \mathscr{E}$ and $a \in A$ by definition of $\delta_{\mathscr{E}}$. The relation $\beta_{\mathscr{E}}\left(n^{\mathrm{o}}\right) \circ \gamma(a)=\gamma\left(\beta_{A}\left(n^{\mathrm{o}}\right) a\right)$ for $n \in N$ and $a \in A$ is straightforward.

From now on, we assume the quantum groupoid $\mathcal{G}$ to be regular. We recall that any action of the quantum groupoid $\mathcal{G}$ on a Hilbert module is necessarily continuous (cf. 6.1.26).

7.11 Proposition-Definition. - Let $A, C$ and $B$ be $\mathcal{G}$-C*algebras. Let $\mathscr{E}_{1}\left(\right.$ resp. $\mathscr{E}_{2}$ ) be a $\mathcal{G}$-equivariant imprimitivity $A$-C-bimodule (resp. $C$-B-bimodule). Denote by $\mathscr{E}_{1} \otimes_{C} \mathscr{E}_{2}$ the internal tensor product $\mathscr{E}_{1} \otimes_{\gamma_{2}} \mathscr{E}_{2}$, where $\gamma_{2}: C \rightarrow \mathcal{L}\left(\mathscr{E}_{2}\right)$ is the $\mathcal{G}$-equivariant ${ }^{*}$-representation defined by the left action of $C$ on $\mathscr{E}_{2}$. The Hilbert $B$-module $\mathscr{E}_{1} \otimes_{C} \mathscr{E}_{2}$ endowed with the action of $\mathcal{G}$ defined in 7.9 is a $\mathcal{G}$-equivariant imprimitivity $A$-B-bimodule for the left action of $A$ and the A-valued inner product defined by the formulas:

- $a\left(\xi_{1} \otimes_{C} \xi_{2}\right):=a \xi_{1} \otimes_{C} \xi_{2}$, for all $a \in A, \xi_{1} \in \mathscr{E}_{1}$ and $\xi_{2} \in \mathscr{E}_{2}$;

- ${ }_{A}\left\langle\xi_{1} \otimes_{C} \xi_{2}, \eta_{1} \otimes_{C} \eta_{2}\right\rangle:={ }_{A}\left\langle\xi_{1}, \eta_{1} C\left\langle\xi_{2}, \eta_{2}\right\rangle\right\rangle$, for all $\xi_{1}, \eta_{1} \in \mathscr{E}_{1}$ and $\xi_{2}, \eta_{2} \in \mathscr{E}_{2}$.

Proof. It is known that $\mathscr{E}_{1} \otimes_{C} \mathscr{E}_{2}$ is an imprimitivity $A$-B-bimodule. The rest of the proof is contained in 7.9 and 7.10 .

7.12 Proposition. - Let $A$ and $B$ be $\mathcal{G}$-C $C^{*}$-algebras. Let $\mathscr{E}$ be a $\mathcal{G}$-equivariant imprimitivity $A$-B-bimodule. Then, the map $\mathscr{E}^{*} \otimes_{A} \mathscr{E} \rightarrow B ; \xi^{*} \otimes_{A} \eta \mapsto\langle\xi, \eta\rangle_{B}$ defines an isomorphism of $\mathcal{G}$-equivariant imprimitivity $B$-B-bimodules.

Proof. It is known that the map $\Phi: \mathscr{E}^{*} \otimes_{A} \mathscr{E} \rightarrow B ; \xi^{*} \otimes_{A} \eta \mapsto\langle\xi, \eta\rangle_{B}$ is an isomorphism of imprimitivity $B$-B-bimodules. The $\mathcal{G}$-equivariance of $\Phi$ is a restatement of the formula $\delta_{B}\left(\langle\xi, \eta\rangle_{B}\right)=\delta_{\mathscr{E}}(\xi)^{*} \circ \delta_{\mathscr{E}}(\eta)$ for $\xi, \eta \in \mathscr{E}$.

7.13 Definition. - Let $A$ and $B$ be $\mathcal{G}$ - $C^{*}$-algebras. We say that $A$ and $B$ are $\mathcal{G}$-equivariantly Morita equivalent if there exists a $\mathcal{G}$-equivariant imprimitivity $A$-B-bimodule. The $\mathcal{G}$ equivariant Morita equivalence is a reflexive $77.7(\mathrm{i}))$, symmetric $[7.7$ (iv)) and transitive 7.11 relation on the class of $\mathcal{G}$ - $\mathrm{C}^{*}$-algebras.

BIDUALITY AND EQUIVARIANT MORITA EQUIVALENCE. In this paragraph, the measured quantum groupoid $\mathcal{G}$ is assumed to be regular. Let us fix a $\mathcal{G}$-C*-algebras $A$. We show that there is a canonical $\mathcal{G}$-equivariant Morita equivalence between $A$ and the double crossed product $(A \rtimes \mathcal{G}) \rtimes \widehat{\mathcal{G}}$.

7.14 Notations. - Denote by $\mathcal{K}:=\mathcal{K}(\mathscr{H})$ for short. Consider the Hilbert $A$-modules $\mathcal{E}_{0}:=A \otimes \mathscr{H}$ and $\mathcal{E}_{A, R}:=q_{\beta_{A} \widehat{\alpha}}(A \otimes \mathscr{H})$. Let $\mathcal{V} \in \mathcal{L}(\mathscr{H} \otimes S)$ be the unique partial isometry such that $\left(\operatorname{id}_{\mathcal{K}} \otimes L\right)(\mathcal{V})=V$.

7.15 Proposition. - There exists a unique bounded linear map $\delta_{\mathcal{E}_{0}}: \mathcal{E}_{0} \rightarrow \mathcal{L}\left(A \otimes S, \mathcal{E}_{0} \otimes S\right)$ such that $\delta_{\mathcal{E}_{0}}(a \otimes \zeta)=\mathcal{V}_{23} \delta_{A}(a)_{13}\left(1_{A} \otimes \zeta \otimes 1_{S}\right)$, for all $a \in A$ and $\zeta \in \mathscr{H}$. 
Proof. If $B$ is a $C^{*}$-algebra and $\mathscr{K}$ a Hilbert space, we identify $\mathcal{M}(B) \otimes \mathscr{K}$ with a closed vector subspace of $\mathcal{L}(B, B \otimes \mathscr{K})$. We have $\left(\delta_{A} \otimes \operatorname{id}_{\mathscr{H}}\right)(\xi) \in \mathcal{L}(A \otimes S, A \otimes S \otimes \mathscr{H})$ and $\left(\delta_{A} \otimes \operatorname{id}_{\mathscr{H}}\right)(\xi)^{*}=\left(\delta_{A} \otimes \operatorname{id}_{\mathscr{H}^{*}}\right)\left(\xi^{*}\right)$ for $\xi \in \mathcal{E}_{0}$. Let $\sigma \in \mathcal{L}(S \otimes \mathscr{H}, \mathscr{H} \otimes S)$ be the flip map. Denote by $\delta_{\mathcal{E}_{0}}: \mathcal{E}_{0} \rightarrow \mathcal{L}\left(A \otimes S, \mathcal{E}_{0} \otimes S\right)$ the map defined by $\delta_{\mathcal{E}_{0}}(\xi):=\mathcal{V}_{23} \sigma_{23}\left(\delta_{A} \otimes \mathrm{id} \mathscr{H}\right)(\xi)$ for $\xi \in \mathcal{E}_{0}$. It is clear that $\delta_{\mathcal{E}_{0}}: \mathcal{E}_{0} \rightarrow \mathcal{L}\left(A \otimes S, \mathcal{E}_{0} \otimes S\right)$ is linear map satsifying the formula $\delta_{\mathcal{E}_{0}}(a \otimes \xi)=\mathcal{V}_{23} \delta_{A}(a)_{13}\left(1_{A} \otimes \xi \otimes 1_{S}\right)$ for all $a \in A$ and $\zeta \in \mathscr{H}$.

7.16 Proposition. - We have the following statements:

1. $\delta_{\mathcal{E}_{0}}(\xi)^{*} \delta_{\mathcal{E}_{0}}(\eta)=\delta_{A}\left(\left\langle q_{\beta_{A} \widehat{\alpha}} \xi, q_{\beta_{A} \widehat{\alpha}} \eta\right\rangle\right)$, for all $\xi, \eta \in \mathcal{E}_{0}$;

2. $\delta_{\mathcal{E}_{0}}(\xi a)=\delta_{\mathcal{E}_{0}}(\xi) \delta_{A}(a)$, for all $\xi \in \mathcal{E}_{0}$ and $a \in A$;

3. $\delta_{\mathcal{E}_{0}}\left(q_{\beta_{A} \widehat{\alpha}} \xi\right)=\delta_{\mathcal{E}_{0}}(\xi)$, for all $\xi \in \mathcal{E}_{0}$;

4. $\delta_{\mathcal{E}_{0}}\left(\mathcal{E}_{0}\right)(A \otimes S) \subset \mathcal{E}_{A, R} \otimes S$.

Proof. 1. Let $\xi, \eta \in \mathcal{E}_{0}$, we have $\delta_{\mathcal{E}_{0}}(\xi)^{*} \delta_{\mathcal{E}_{0}}(\eta)=\left(\delta_{A} \otimes \mathrm{id}\right)\left(\xi^{*}\right) \sigma_{23}^{*} \mathcal{V}_{23}^{*} \mathcal{V}_{23} \sigma_{23}\left(\delta_{A} \otimes \mathrm{id}\right)(\eta)$. We have $\sigma^{*} \mathcal{V}^{*} \mathcal{V} \sigma=q_{\beta \widehat{\alpha}}$. Let $n, n^{\prime} \in N$. For all $a \in A$ and $\zeta \in \mathscr{H}$, we have

$$
\begin{aligned}
\left(1_{A} \otimes \beta\left(n^{\mathrm{o}}\right) \otimes \widehat{\alpha}\left(n^{\prime}\right)\right)\left(\delta_{A} \otimes \mathrm{id}_{\mathscr{H}}\right)(a \otimes \zeta) & =\left(1_{A} \otimes \beta\left(n^{\mathrm{o}}\right)\right) \delta_{A}(a) \otimes \widehat{\alpha}\left(n^{\prime}\right) \zeta \\
& =\delta_{A}\left(\beta_{A}\left(n^{\mathrm{o}}\right) a\right) \otimes \widehat{\alpha}\left(n^{\prime}\right) \zeta \\
& =\left(\delta_{A} \otimes \operatorname{id}_{\mathscr{H}}\right)\left(\left(\beta_{A}\left(n^{\mathrm{o}}\right) \otimes \widehat{\alpha}\left(n^{\prime}\right)\right)(a \otimes \zeta)\right) .
\end{aligned}
$$

Hence, $\left(1_{A} \otimes \beta\left(n^{\mathrm{o}}\right) \otimes \widehat{\alpha}\left(n^{\prime}\right)\right)\left(\delta_{A} \otimes \operatorname{id}_{\mathscr{H}}\right)(\eta)=\left(\delta_{A} \otimes \mathrm{id}_{\mathscr{H}}\right)\left(\left(\beta_{A}\left(n^{\mathrm{o}}\right) \otimes \widehat{\alpha}\left(n^{\prime}\right)\right) \eta\right)$. It then follows that $\sigma_{23}^{*} \mathcal{V}_{23}^{*} \mathcal{V}_{23} \sigma_{23}\left(\delta_{A} \otimes \operatorname{id}_{\mathscr{H}}\right)(\eta)=\left(\delta_{A} \otimes \operatorname{id}_{\mathscr{H}}\right)\left(q_{\beta_{A} \widehat{\alpha}} \eta\right)$. We finally have

$$
\begin{aligned}
\delta_{\mathcal{E}_{0}}(\xi)^{*} \delta_{\mathcal{E}_{0}}(\eta) & =\left(\delta_{A} \otimes \operatorname{id}_{\mathscr{H}^{*}}\right)\left(\xi^{*}\right)\left(\delta_{A} \otimes \operatorname{id}_{\mathscr{H}}\right)\left(q_{\beta_{A} \widehat{\alpha}} \eta\right) \\
& =\delta_{A}\left(\left\langle\xi, q_{\beta_{A} \widehat{\alpha}} \eta\right\rangle\right) \\
& =\delta_{A}\left(\left\langle q_{\beta_{A} \widehat{\alpha}} \xi, q_{\beta_{A} \widehat{\alpha}} \eta\right\rangle\right),
\end{aligned}
$$

where the last equality follows from the fact that $q_{\beta_{A} \widehat{\alpha}} \in \mathcal{L}\left(\mathcal{E}_{0}\right)$ is a self-adjoint projection. 2. Let us fix $a, b \in A$ and $\zeta \in \mathscr{H}$. We have $\delta_{A}(a){ }_{13}\left(1_{A} \otimes \zeta \otimes 1_{S}\right)=\left(1_{A} \otimes \zeta \otimes 1_{S}\right) \delta_{A}(a)$ in $\mathcal{L}\left(A \otimes S, \mathcal{E}_{0} \otimes S\right)$. Hence, $\delta_{\mathcal{E}_{0}}((b \otimes \zeta) a)=\delta_{\mathcal{E}_{0}}(b \otimes \zeta) \delta_{A}(a)$.

3. Let $a \in A$ and $\zeta \in \mathscr{H}$. For all $n, n^{\prime} \in N$, we have

$$
\begin{aligned}
\delta_{\mathcal{E}_{0}}\left(\beta_{A}\left(n^{\mathrm{o}}\right) a \otimes \widehat{\alpha}\left(n^{\prime}\right) \zeta\right) & =\mathcal{V}_{23}\left(1_{A} \otimes \widehat{\alpha}\left(n^{\prime}\right) \otimes 1_{S}\right) \delta_{A}\left(\beta_{A}\left(n^{\mathrm{o}}\right) a\right)_{13}\left(1_{A} \otimes \zeta \otimes 1_{S}\right) \\
& =\mathcal{V}_{23}\left(1_{A} \otimes \widehat{\alpha}\left(n^{\prime}\right) \otimes \beta\left(n^{\mathrm{o}}\right)\right) \delta_{A}(a)_{13}\left(1_{A} \otimes \zeta \otimes 1_{S}\right) .
\end{aligned}
$$

Hence, $\delta_{\mathcal{E}_{0}}\left(q_{\beta_{A} \widehat{\alpha}}(a \otimes \zeta)\right)=\mathcal{V}_{23} q_{\widehat{\alpha} \beta, 23} \delta_{A}(a)_{13}\left(1_{A} \otimes \zeta \otimes 1_{S}\right)=\delta_{\mathcal{E}_{0}}(a \otimes \zeta)$.

4. It suffices to show that $q_{\beta_{A} \widehat{\alpha}, 12} \delta_{\mathcal{E}_{0}}(\xi)=\delta_{\mathcal{E}_{0}}(\xi)$ for all $\xi \in \mathcal{E}_{0}$. We recall (cf. 3.1.5 that $\left(\widehat{\alpha}(n) \otimes 1_{S}\right) \mathcal{V}=\mathcal{V}\left(1_{\mathcal{K}} \otimes \alpha(n)\right)$ for all $n \in N$. Hence, $q_{\beta_{A} \widehat{\alpha}, 12} \mathcal{V}_{23}=\mathcal{V}_{23} q_{\beta_{A} \alpha, 13}$. It then follows from $q_{\beta_{A} \alpha}=\delta_{A}\left(1_{A}\right)$ that $q_{\beta_{A} \widehat{\alpha}, 12} \mathcal{V}_{23} \delta_{A}(a)_{13}=\mathcal{V}_{23} \delta_{A}(a)_{13}$ for all $a \in A$. Hence, $q_{\beta_{A} \widehat{\alpha}, 12} \delta_{\mathcal{E}_{0}}(a \otimes \zeta)=\delta_{\mathcal{E}_{0}}(a \otimes \zeta)$ for all $a \in A$ and $\zeta \in \mathscr{H}$.

7.17 Notations. - According to the previous proposition, $\delta_{\mathcal{E}_{0}}$ restricts to a linear map

$$
\delta_{\mathcal{E}_{A, R}}: \mathcal{E}_{A, R} \rightarrow \mathcal{L}\left(A \otimes S, \mathcal{E}_{A, R} \otimes S\right),
$$

which satisfies the following statements:

- $\delta_{\mathcal{E}_{A, R}}(\xi)^{*} \delta_{\mathcal{E}_{A, R}}(\eta)=\delta_{A}(\langle\xi, \eta\rangle)$, for all $\xi, \eta \in \mathcal{E}_{A, R} ;$

- $\delta_{\mathcal{E}_{A, R}}(\xi a)=\delta_{\mathcal{E}_{A, R}}(\xi) \delta_{A}(a)$, for all $\xi \in \mathcal{E}_{A, R}$ and $a \in A$.

Since $\left[\widehat{\alpha}\left(n^{\prime}\right), \beta\left(n^{\mathrm{o}}\right)\right]=0$ for all $n, n^{\prime} \in N$, we have $\left[1_{A} \otimes \beta\left(n^{\mathrm{o}}\right), q_{\beta_{A} \widehat{\alpha}}\right]=0$ for all $n \in N$. We then have a non-degenerate ${ }^{*}$-homomorphism

$$
\beta_{\mathcal{E}_{A, R}}: N^{\mathrm{o}} \rightarrow \mathcal{L}\left(\mathcal{E}_{A, R}\right) ;\left.n \mapsto\left(1_{A} \otimes \beta\left(n^{\mathrm{o}}\right)\right)\right|_{\mathcal{E}_{A, R}} .
$$

Since $\beta$ and $\widehat{\alpha}$ commute pointwise and $\mathcal{V} \mathcal{V}^{*}=q_{\beta \alpha}$, we have $\left[\mathcal{V}_{23} \mathcal{V}_{23}^{*}, q_{\beta_{A} \widehat{\alpha}, 12}\right]=0$. Hence, $\left.q_{\beta_{\mathcal{E}_{A, R} \alpha}}=\mathcal{V}_{23} \mathcal{V}_{23}^{*}\right\rceil_{\mathcal{E}_{A, R} \otimes S} \in \mathcal{L}\left(\mathcal{E}_{A, R} \otimes S\right)$. 
7.18 Proposition. - We have the following statements:

1. $\delta_{\mathcal{E}_{A, R}}\left(\mathcal{E}_{A, R}\right) \subset \widetilde{\mathcal{M}}\left(\mathcal{E}_{A, R} \otimes S\right) ;$

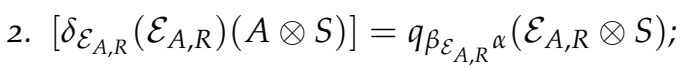

3. $\delta_{\mathcal{E}_{A, R}}\left(\beta_{\mathcal{E}_{A, R}}\left(n^{\mathrm{o}}\right) \xi\right)=\left(1_{\mathcal{E}_{A, R}} \otimes \beta\left(n^{\mathrm{o}}\right)\right) \delta_{\mathcal{E}_{A, R}}(\xi)$, for all $\xi \in \mathcal{E}_{A, R}$ and $n \in N$.

Proof. 1. Let us prove that $\delta_{\mathcal{E}_{A, R}}(\xi)\left(1_{A} \otimes s\right) \in \mathcal{E}_{A, R} \otimes S$ for all $\xi \in \mathcal{E}_{A, R}$ and $s \in S$. It amounts to proving that $\delta_{\mathcal{E}_{0}}(\xi)\left(1_{A} \otimes s\right) \in \mathcal{E}_{0} \otimes S$ for all $\xi \in \mathcal{E}_{0}$ and $s \in S$ (cf. 7.16 3, 4). Let $a \in A$ and $\zeta \in \mathscr{H}$. It follows from the relation $\delta_{A}(A)\left(1_{A} \otimes S\right) \subset A \otimes S$ that $\delta_{\mathcal{E}_{0}}(a \otimes \zeta)\left(1_{A} \otimes s\right)=\left(1_{A} \otimes \mathcal{V}\left(\zeta \otimes 1_{S}\right)\right) \delta_{A}(a)\left(1_{A} \otimes s\right)$ is the norm limit of finite sums of the form $\sum_{i} a_{i} \otimes \mathcal{V}\left(\zeta \otimes s_{i}\right)$, where $a_{i} \in A$ and $s_{i} \in S$. Hence, $\delta_{\mathcal{E}_{0}}(a \otimes \zeta)\left(1_{A} \otimes s\right) \in \mathcal{E}_{0} \otimes S$.

Now, let us prove that $\left(1_{\mathcal{E}_{A, R}} \otimes y\right) \delta_{\mathcal{E}_{A, R}}(\xi) \in \mathcal{E}_{A, R} \otimes S$ for all $\xi \in \mathcal{E}_{A, R}$ and $y \in S$. This also amounts to proving that $\left(1_{\mathcal{E}_{0}} \otimes y\right) \delta_{\mathcal{E}_{0}}(\xi) \in \mathcal{E}_{0} \otimes S$ for all $\xi \in \mathcal{E}_{0}$ and $y \in S$. Let $a \in A, \zeta \in \mathscr{H}$ and $y \in S$, we have $\left(1_{\mathcal{E}_{0}} \otimes y\right) \delta_{\mathcal{E}_{0}}(a \otimes \zeta)=\left(1_{A} \otimes\left(1_{\mathscr{H}} \otimes y\right) \mathcal{V}\left(\zeta \otimes 1_{S}\right)\right) \delta_{A}(a)$. Write $\zeta=\rho(x) \eta$ with $x \in \widehat{S}$ and $\eta \in \mathscr{H}$. We have $\left(1_{\mathcal{K}} \otimes y\right) \mathcal{V}\left(\zeta \otimes 1_{S}\right)=\left(\rho \otimes \mathrm{id}_{S}\right)\left(\left(1_{\widehat{S}} \otimes y\right) V\left(x \otimes 1_{S}\right)\right)\left(\eta \otimes 1_{S}\right)$. Since $\mathcal{G}$ is regular, we have $\left(1_{\widehat{S}} \otimes y\right) V\left(x \otimes 1_{S}\right) \in \widehat{S} \otimes S$ (cf. 4.10 2). Hence, $\left(1_{\mathcal{E}_{0}} \otimes y\right) \delta_{\mathcal{E}_{0}}(a \otimes \zeta)$ is the norm limit of finite sums of elements of the form $\left(1_{A} \otimes \rho\left(x^{\prime}\right) \eta \otimes y^{\prime}\right) \delta_{A}(a)$ with $x^{\prime} \in \widehat{S}$ and $y^{\prime} \in S$. Hence, $\left(1_{\mathcal{E}_{0}} \otimes y\right) \delta_{\mathcal{E}_{0}}(a \otimes \zeta) \in \mathcal{E}_{0} \otimes S$ since $\left(1_{A} \otimes S\right) \delta_{A}(A) \subset A \otimes S$.

2. Since $\mathcal{V} \mathcal{V}^{*} \mathcal{V}=\mathcal{V}$, we have $\mathcal{V}_{23} \mathcal{V}_{23}^{*} \delta_{\mathcal{E}_{0}}(\xi)=\delta_{\mathcal{E}_{0}}(\xi)$ for all $\xi \in \mathcal{E}_{0}$. It then follows that

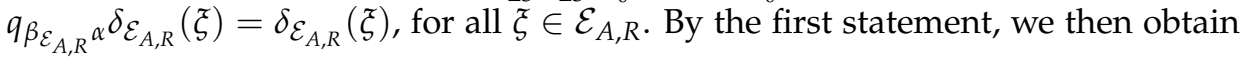

$$
\delta_{\mathcal{E}_{A, R}}\left(\mathcal{E}_{A, R}\right)(A \otimes S) \subset q_{\beta_{\mathcal{E}_{A, R} \alpha}\left(\mathcal{E}_{A, R} \otimes S\right)}
$$

Conversely, let $a \in A, \zeta \in \mathscr{H}$ and $s \in S$. Since $\mathcal{V}_{23} q_{\beta_{A} \alpha, 13}=q_{\beta_{A} \widehat{\alpha}, 12} \mathcal{V}_{23}$, we have

$$
q_{\beta_{\mathcal{E}_{A, R} \alpha}}\left(q_{\beta_{A} \widehat{\alpha}}(a \otimes \zeta) \otimes s\right)=\mathcal{V}_{23} \mathcal{V}_{23}^{*} q_{\beta_{A} \widehat{\alpha}, 12}(a \otimes \zeta \otimes s)=\mathcal{V}_{23} q_{\beta_{A} \alpha, 13}\left(a \otimes \mathcal{V}^{*}(\zeta \otimes s)\right)
$$

Hence, $q_{\beta_{\mathcal{E}_{A, R} \alpha}}\left(q_{\beta_{A} \widehat{\alpha}}(a \otimes \zeta) \otimes s\right)$ is the norm limit of finite sums of elements of the form:

$$
\mathcal{V}_{23} q_{\beta_{A} \alpha, 13}\left(a \otimes \zeta^{\prime} \otimes s^{\prime}\right)=\mathcal{V}_{23}\left(q_{\beta_{A} \alpha}\left(a \otimes s^{\prime}\right)\right)_{23}\left(1_{A} \otimes \zeta^{\prime} \otimes 1_{S}\right), \quad \text { where } \zeta^{\prime} \in \mathscr{H}, s^{\prime} \in S \text {. }
$$

By continuity of the action $\left(\delta_{A}, \beta_{A}\right), \mathcal{V}_{23} q_{\beta_{A} \alpha, 13}\left(a \otimes \zeta^{\prime} \otimes s^{\prime}\right)$ is the norm limit of finite sums of the form $\sum_{i} \mathcal{V}_{23} \delta_{A}\left(a_{i}\right)_{13}\left(1_{A} \otimes \zeta^{\prime} \otimes s_{i}\right)=\sum_{i} \delta_{\mathcal{E}_{A, R}}\left(q_{\beta_{A} \widehat{\alpha}}\left(a_{i} \otimes \zeta^{\prime}\right)\right)\left(1_{A} \otimes s_{i}\right)$, where $a_{i} \in A$ and $s_{i} \in S$. As a result, we have

$$
q_{\beta_{\mathcal{E}_{A, R} \alpha} \alpha}\left(q_{\beta_{A} \widehat{\alpha}}(a \otimes \zeta) \otimes s\right) \in\left[\delta_{\mathcal{E}_{A, R}}\left(\mathcal{E}_{A, R}\right)(A \otimes S)\right]
$$

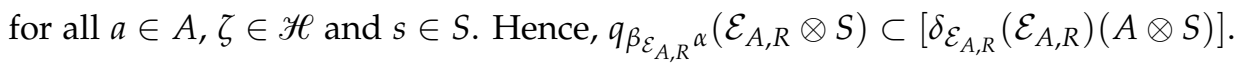

3. Let $\xi=q_{\beta_{A} \widehat{\alpha}}(a \otimes \zeta)$, with $a \in A$ and $\zeta \in \mathscr{H}$. We have

$$
\beta_{\mathcal{E}_{A, R}}\left(n^{\mathrm{o}}\right) \xi=\left(1_{A} \otimes \beta\left(n^{\mathrm{o}}\right)\right) q_{\beta_{A} \widehat{\alpha}}(a \otimes \zeta)=q_{\beta_{A} \widehat{\alpha}}\left(a \otimes \beta\left(n^{\mathrm{o}}\right) \zeta\right) .
$$

Moreover, we have $\mathcal{V}\left(\beta\left(n^{\mathrm{o}}\right) \otimes 1_{S}\right)=\left(1_{\mathcal{K}} \otimes \beta\left(n^{\mathrm{o}}\right)\right) \mathcal{V}$ for all $n \in N$ (cf. 3.1.5). It then follows that

$$
\delta_{\mathcal{E}_{A, R}}\left(\beta_{\mathcal{E}_{A, R}}\left(n^{\mathrm{o}}\right) \xi\right)=\delta_{\mathcal{E}_{0}}\left(a \otimes \beta\left(n^{\mathrm{o}}\right) \zeta\right)=\left(1_{\mathcal{E}_{0}} \otimes \beta\left(n^{\mathrm{o}}\right)\right) \delta_{\mathcal{E}_{0}}(a \otimes \zeta)=\left(1_{\mathcal{E}_{A, R}} \otimes \beta\left(n^{\mathrm{o}}\right)\right) \delta_{\mathcal{E}_{A, R}}(\xi) .
$$

Consequently, $\delta_{\mathcal{E}_{A, R}} \otimes \mathrm{id}_{S}$ and $\mathrm{id}_{\mathcal{E}_{A R}} \otimes \delta$ extend to linear maps from $\mathcal{L}\left(A \otimes S, \mathcal{E}_{A, R} \otimes S\right)$ to $\mathcal{L}\left(A \otimes S \otimes S, \mathcal{E}_{A, R} \otimes S \otimes S\right)$ (cf. 6.1.7) and we have:

$$
\begin{gathered}
\left(\delta_{\mathcal{E}_{A, R}} \otimes \mathrm{id}_{S}\right)(T)\left(\delta_{A} \otimes \mathrm{id}_{S}\right)(x)=\left(\delta_{\mathcal{E}_{A, R}} \otimes \mathrm{id}_{S}\right)(T x) ; \\
\left(\mathrm{id}_{\mathcal{E}_{A, R}} \otimes \delta\right)(T)\left(\mathrm{id}_{A} \otimes \delta\right)(x)=\left(\mathrm{id}_{\mathcal{E}_{A, R}} \otimes \delta\right)(T x) ;
\end{gathered}
$$

for all $x \in A \otimes S$ and $T \in \mathcal{L}\left(A \otimes S, \mathcal{E}_{A, R} \otimes S\right)$.

7.19 Proposition. - For all $\xi \in \mathcal{E}_{A, R},\left(\delta_{\mathcal{E}_{A, R}} \otimes \mathrm{id}_{S}\right) \delta_{\mathcal{E}_{A, R}}(\xi)=\left(\mathrm{id}_{\mathcal{E}_{A, R}} \otimes \delta\right) \delta_{\mathcal{E}_{A, R}}(\xi)$. 
Proof. Let $a \in A, \zeta \in \mathscr{H}$ and $x \in A \otimes S$. Let $\xi:=q_{\beta_{A} \widehat{\alpha}}(a \otimes \zeta)$. We have

$$
\left(\delta_{\mathcal{E}_{A, R}} \otimes \mathrm{id}_{S}\right) \delta_{\mathcal{E}_{A, R}}(\xi)\left(\delta_{A} \otimes \mathrm{id}_{S}\right)(x)=\left(\delta_{\mathcal{E}_{0}} \otimes \operatorname{id}_{S}\right)\left(\mathcal{V}_{23}\left(\delta_{A}(a) x\right)_{13}\left(1_{A} \otimes \zeta \otimes 1_{S}\right)\right) .
$$

For all $b \in A, \zeta^{\prime} \in \mathscr{H}$ and $s^{\prime} \in S$, we have

$$
\left(\delta_{\mathcal{E}_{0}} \otimes \mathrm{id}_{S}\right)\left(b \otimes \zeta^{\prime} \otimes s^{\prime}\right)=\mathcal{V}_{23} \delta_{A}(b)_{13}\left(1_{A} \otimes \zeta^{\prime} \otimes 1_{S} \otimes s^{\prime}\right) .
$$

Hence, $\left(\delta_{\mathcal{E}_{0}} \otimes \mathrm{id}_{S}\right)(b \otimes X)=\mathcal{V}_{23} \delta_{A}(b)_{13} X_{24} \in \mathcal{L}(A \otimes S \otimes S, A \otimes \mathscr{H} \otimes S \otimes S)$ for all $b \in A$ and $X \in \mathscr{H} \otimes S$. In particular, we have

$$
\begin{aligned}
\left(\delta_{\mathcal{E}_{0}} \otimes \mathrm{id}_{S}\right)\left(\mathcal{V}_{23}(b \otimes \zeta \otimes s)\right) & =\left(\delta_{\mathcal{E}_{0}} \otimes \mathrm{id}_{S}\right)(b \otimes \mathcal{V}(\zeta \otimes s)) \\
& =\mathcal{V}_{23} \delta_{A}(b)_{13} \mathcal{V}_{24}\left(1_{A} \otimes \zeta \otimes 1_{S} \otimes s\right) \\
& =\mathcal{V}_{23} \mathcal{V}_{24} \delta_{A}(b)_{13}\left(1_{A} \otimes \zeta \otimes 1_{S} \otimes s\right) .
\end{aligned}
$$

However, we have $\left(\operatorname{id}_{\mathcal{K}} \otimes \delta\right)(\mathcal{V})=\mathcal{V}_{12} \mathcal{V}_{13}$. Hence, $\mathcal{V}_{23} \mathcal{V}_{24}=\left(\mathrm{id}_{A \otimes \mathcal{K}} \otimes \delta\right)\left(\mathcal{V}_{23}\right)$. Moreover, we have $\delta_{A}(b)_{13}\left(1_{A} \otimes \zeta \otimes 1_{S} \otimes s\right)=\left(\delta_{A, 13} \otimes \mathrm{id}_{S}\right)(b \otimes s)\left(1_{A} \otimes \zeta \otimes 1_{S} \otimes 1_{S}\right)$, for all $b \in A$ and $s \in S$, where $\delta_{A, 13}: A \rightarrow \mathcal{M}(A \otimes \mathcal{K} \otimes S)$ is the strictly continuous ${ }^{*}$-homomorphism defined by $\delta_{A, 13}(a):=\delta_{A}(a)_{13}$ for all $a \in A$. As a result, for all $Y \in A \otimes S$ we have

$$
\left(\delta_{\mathcal{E}_{0}} \otimes \mathrm{id}_{S}\right)\left(\mathcal{V}_{23} Y_{13}\left(1_{A} \otimes \zeta \otimes 1_{S}\right)\right)=\left(\mathrm{id}_{A \otimes \mathcal{K}} \otimes \delta\right)\left(\mathcal{V}_{23}\right)\left(\delta_{A, 13} \otimes \mathrm{id}_{S}\right)(Y)\left(1_{A} \otimes \zeta \otimes 1_{S} \otimes 1_{S}\right) .
$$

In particular, we have

$$
\begin{array}{r}
\left(\delta_{\mathcal{E}_{0}} \otimes \mathrm{id}_{S}\right)\left(\delta_{\mathcal{E}_{0}}(a \otimes \zeta) x\right)=\left(\operatorname{id}_{A \otimes \mathcal{K}} \otimes \delta\right)\left(\mathcal{V}_{23}\right)\left(\delta_{A, 13} \otimes \mathrm{id}_{S}\right)\left(\delta_{A}(a) x\right)\left(1_{A} \otimes \zeta \otimes 1_{S} \otimes 1_{S}\right) \\
=\left(\operatorname{id}_{A \otimes \mathcal{K}} \otimes \delta\right)\left(\mathcal{V}_{23}\right)\left(\delta_{A, 13} \otimes \mathrm{id}_{S}\right) \delta_{A}(a)\left(1_{A} \otimes \zeta \otimes 1_{S} \otimes 1_{S}\right)\left(\delta_{A} \otimes \mathrm{id}_{S}\right)(x) .
\end{array}
$$

Moreover, we have $\left(\delta_{A, 13} \otimes \operatorname{id}_{S}\right) \delta_{A}=\left(\operatorname{id}_{A \otimes \mathcal{K}} \otimes \delta\right) \delta_{A, 13}$. Hence,

$$
\left(\delta_{\mathcal{E}_{A, R}} \otimes \mathrm{id}_{S}\right) \delta_{\mathcal{E}_{A, R}}(\xi) x=\left(\operatorname{id}_{A \otimes \mathcal{K}} \otimes \delta\right)\left(\mathcal{V}_{23} \delta_{A}(a)_{13}\right)\left(1_{A} \otimes \zeta \otimes 1_{S} \otimes 1_{S}\right) x,
$$

for all $x \in q_{\beta_{A} \alpha, 12}(A \otimes S \otimes S)$. In particular, if $x \in q_{\beta_{A} \alpha, 12} q_{\beta \alpha, 23}(A \otimes S \otimes S)$ we have

$$
\begin{aligned}
\left(\delta_{\mathcal{E}_{A, R}} \otimes \operatorname{id}_{S}\right) \delta_{\mathcal{E}_{A, R}}(\xi) x & =\left(\operatorname{id}_{A \otimes \mathcal{K}} \otimes \delta\right)\left(\mathcal{V}_{23} \delta_{A}(a)_{13}\right) q_{\beta \alpha, 34}\left(1_{A} \otimes \zeta \otimes 1_{S} \otimes 1_{S}\right) x \\
& =\left(\operatorname{id}_{A \otimes \mathcal{K}} \otimes \delta\right)\left(\mathcal{V}_{23} \delta_{A}(a)_{13}\right)\left(\operatorname{id}_{\mathcal{E}_{0}} \otimes \delta\right)\left(1_{A} \otimes \zeta \otimes 1_{S}\right) x \\
& =\left(\operatorname{id}_{\mathcal{E}_{0}} \otimes \delta\right) \delta_{\mathcal{E}_{0}}(a \otimes \zeta) x \\
& =\left(\operatorname{id}_{\mathcal{E}_{A, R}} \otimes \delta\right) \delta_{\mathcal{E}_{A, R}}(\xi) x .
\end{aligned}
$$

Hence, $\left(\delta_{\mathcal{E}_{A, R}} \otimes \operatorname{id}_{S}\right) \delta_{\mathcal{E}_{A, R}}(\xi)=\left(\operatorname{id}_{\mathcal{E}_{A, R}} \otimes \delta\right) \delta_{\mathcal{E}_{A, R}}(\xi)$.

Now, we can assemble the previous results (see also 6.1.26) in the statement below.

7.20 Proposition. - The triple $\left(\mathcal{E}_{A, R}, \beta_{\mathcal{E}_{A, R}}, \delta_{\mathcal{E}_{A, R}}\right)$ is a $\mathcal{G}$-equivariant Hilbert A-module.

Let $D$ be the bidual $\mathcal{G}$ - $\mathrm{C}^{*}$-algebra of $A$. We have a canonical $\mathcal{G}$-equivariant ${ }^{*}$-isomorphism $\phi:(A \rtimes \mathcal{G}) \rtimes \widehat{\mathcal{G}} \rightarrow D$ of $\mathcal{G}$-C*-algebras (cf. 5.1.22. Let $j_{D}: \mathcal{M}(D) \rightarrow \mathcal{L}\left(\mathcal{E}_{0}\right)$ be the unique faithful continuous ${ }^{*}$-homomorphism for the strict $/{ }^{*}$-strong topologies such that $j_{D}\left(1_{D}\right)=q_{\beta_{A} \widehat{\alpha}}$.

7.21 Proposition. - The * representation $(A \rtimes \mathcal{G}) \rtimes \widehat{\mathcal{G}} \rightarrow \mathcal{L}\left(\mathcal{E}_{A, R}\right) ; x \mapsto \phi(x) \uparrow_{\mathcal{E}_{A, R}}$ is $\mathcal{G}$ equivariant.

Proof. We have to prove that $\delta_{\mathcal{E}_{0}}(d \xi)=\left(j_{D} \otimes \operatorname{id}_{S}\right)\left(\delta_{D}(d)\right) \circ \delta_{\mathcal{E}_{0}}(\xi)$ for all $d \in D$ and $\xi \in \mathcal{E}_{0}$ (cf. 7.33 and 7.163 ). Let us prove it in three steps:

- Let $b \in A, x \in S$ and $\zeta \in \mathscr{H}$. We have

$$
\delta_{\mathcal{E}_{0}}(b \otimes \lambda(x) \zeta)=\left(1_{A} \otimes \mathcal{V}\left(\lambda(x) \otimes 1_{S}\right)\right) \delta_{A}(b)_{13}\left(1_{A} \otimes \zeta \otimes 1_{S}\right) .
$$

However, $\left[\mathcal{V}, \lambda(x) \otimes 1_{S}\right]=0\left(\right.$ as $\lambda(\widehat{S}) \subset \widehat{M}$ and $\left.V \in \widehat{M}^{\prime} \otimes M\right)$. Hence,

$$
\delta_{\mathcal{E}_{0}}(b \otimes \lambda(x) \zeta)=\left(1_{A} \otimes \lambda(x) \otimes 1_{S}\right) \delta_{\mathcal{E}_{0}}(b \otimes \zeta)
$$


and then $\delta_{\mathcal{E}_{0}}\left(\left(1_{A} \otimes \lambda(x)\right) \xi\right)=\left(1_{A} \otimes \lambda(x) \otimes 1_{S}\right) \delta_{\mathcal{E}_{0}}(\xi)$, for all $x \in \widehat{S}$ and $\xi \in \mathcal{E}_{0}$.

- Let $y \in S$. Since $L(y) \in M \subset \widehat{\alpha}(N)^{\prime}$, we have

$\mathcal{V}\left(L(y) \otimes 1_{S}\right)=\mathcal{V} q_{\widehat{\alpha} \beta}\left(L(y) \otimes 1_{S}\right)=\mathcal{V}\left(L(y) \otimes 1_{S}\right) q_{\widehat{\alpha} \beta}=\mathcal{V}\left(L(y) \otimes 1_{S}\right) \mathcal{V}^{*} \mathcal{V}=\left(L \otimes \mathrm{id}_{S}\right) \delta(y) \mathcal{V}$.

For all $b \in A$ and $\zeta \in \mathscr{H}$, we have

$$
\begin{aligned}
\delta_{\mathcal{E}_{0}}\left(\left(1_{A} \otimes L(y)\right)(b \otimes \zeta)\right) & =\left(1_{A} \otimes \mathcal{V}\left(L(y) \otimes 1_{S}\right)\right) \delta_{A}(b){ }_{13}\left(1_{A} \otimes \zeta \otimes 1_{S}\right) \\
& =\left(1_{A} \otimes\left(L \otimes \mathrm{id}_{S}\right) \delta(y)\right) \delta_{\mathcal{E}_{0}}(b \otimes \zeta) .
\end{aligned}
$$

Hence, $\delta_{\mathcal{E}_{0}}\left(\left(1_{A} \otimes L(y)\right) \xi\right)=\left(1_{A} \otimes\left(L \otimes \mathrm{id}_{S}\right) \delta(y)\right) \delta_{\mathcal{E}_{0}}(\xi)$ for all $y \in S$ and $\xi \in \mathcal{E}_{0}$.

In virtue of the first two steps, for all $\xi \in \mathcal{E}_{A, R}$ we have

$$
\delta_{\mathcal{E}_{A, R}}\left(\left(1_{A} \otimes \lambda(x) L(y)\right) \xi\right)=\left(1_{A} \otimes \lambda(x) \otimes 1_{S}\right)\left(1_{A} \otimes\left(L \otimes \operatorname{id}_{S}\right) \delta(y)\right) \delta_{\mathcal{E}_{A, R}}(\xi) .
$$

- Let $s \in S$. We have (cf. 3.1.3

$$
(R(s) \otimes 1) V=(U \otimes 1) \Sigma(1 \otimes L(s)) \Sigma\left(U^{*} \otimes 1\right) V=(U \otimes 1) \Sigma(1 \otimes L(s)) W \Sigma\left(U^{*} \otimes 1\right) .
$$

Besides, $(1 \otimes L(s)) W=(1 \otimes L(s)) W W^{*} W=W W^{*}(1 \otimes L(s)) W=W \delta(s)$ since we have $W W^{*}=q_{\alpha \widehat{\beta}}$ and $L(s) \in M \subset \widehat{\beta}\left(N^{\mathrm{o}}\right)^{\prime}$. Therefore, since $(U \otimes 1) \Sigma W=V(U \otimes 1) \Sigma$ we have $(R(s) \otimes 1) V=V \Sigma(1 \otimes U) \delta(s)\left(1 \otimes U^{*}\right) \Sigma$. Hence, $\left(R(s) \otimes 1_{S}\right) \mathcal{V}=\mathcal{V} \sigma\left(\operatorname{id}_{S} \otimes R\right)(\delta(s)) \sigma^{*}$ for all $s \in S$. We then have $\left(\left(\operatorname{id}_{A} \otimes R\right)(x) \otimes 1_{S}\right) \mathcal{V}_{23}=\mathcal{V}_{23} \sigma_{23}\left(\operatorname{id}_{A \otimes S} \otimes R\right)\left(\left(\operatorname{id}_{A} \otimes \delta\right)(x)\right) \sigma_{23}^{*}$ for all $x \in A \otimes S$. But, since $R$ and $\delta$ are strictly continuous this equality also holds for all $x \in \mathcal{M}(A \otimes S)$. In particular, we have $\pi_{R}(a)_{12} \mathcal{V}_{23}=\mathcal{V}_{23} \sigma_{23}\left(\operatorname{id}_{A \otimes S} \otimes R\right)\left(\delta_{A}^{2}(a)\right) \sigma_{23}^{*}$ for all $a \in A$. By coassociativity of $\delta_{A}$, we have

$$
\pi_{R}(a)_{12} \mathcal{V}_{23}=\mathcal{V}_{23} \sigma_{23}\left(\delta_{A} \otimes \operatorname{id}_{\mathcal{K}}\right)\left(\pi_{R}(a)\right) \sigma_{23}^{*}, \quad \text { for all } a \in A
$$

It then follows that

$$
\begin{aligned}
\pi_{R}(a)_{12} \delta_{\mathcal{E}_{0}}(\xi) & =\mathcal{V}_{23} \sigma_{23}\left(\delta_{A} \otimes \operatorname{id}_{\mathcal{K}}\right)\left(\pi_{R}(a)\right)\left(\delta_{A} \otimes \operatorname{id}_{\mathscr{H}}\right)(\xi) \\
& =\mathcal{V}_{23} \sigma_{23}\left(\delta_{A} \otimes \operatorname{id}_{\mathscr{H}}\right)\left(\pi_{R}(a) \xi\right) \\
& =\delta_{\mathcal{E}_{0}}\left(\pi_{R}(a) \xi\right),
\end{aligned}
$$

for all $a \in A$ and $\xi \in \mathcal{E}_{0}$. In particular, $\pi_{R}(a)_{12} \delta_{\mathcal{E}_{0}}\left(\xi_{0}\right)=\delta_{\mathcal{E}_{0}}\left(\pi_{R}(a) \xi_{0}\right)$ for all $a \in A$ and $\xi \in \mathcal{E}_{0}$.

We have proved that for all $a \in A, x \in \widehat{S}, y \in S$ and $\xi \in \mathcal{E}_{0}$, we have

$$
\delta_{\mathcal{E}_{0}}\left(\pi_{R}(a)\left(1_{A} \otimes \lambda(x) L(y)\right) \xi\right)=\pi_{R}(a)_{12}\left(1_{A} \otimes \lambda(x) \otimes 1_{S}\right)\left(1_{A} \otimes\left(L \otimes \operatorname{id}_{S}\right) \delta(y)\right) \delta_{\mathcal{E}_{0}}(\xi) .
$$

However, for all $a \in A, x \in \widehat{S}$ and $y \in S$ we have (cf. 3.37 d) [2])

$$
\left(j_{D} \otimes \operatorname{id}_{S}\right) \delta_{D}\left(\pi_{R}(a)\left(1_{A} \otimes \lambda(x) L(y)\right)\right)=\pi_{R}(a)_{12}\left(1_{A} \otimes \lambda(x) \otimes 1_{S}\right)\left(1_{A} \otimes\left(L \otimes \mathrm{id}_{S}\right) \delta(y)\right) .
$$

If $d=\pi_{R}(a)\left(1_{A} \otimes \lambda(x) L(y)\right) \in D$, where $a \in A, x \in \widehat{S}$ and $y \in S$, we have proved that $\delta_{\mathcal{E}_{0}}(d \xi)=\left(j_{D} \otimes \operatorname{id}_{S}\right)\left(\delta_{D}(d)\right) \circ \delta_{\mathcal{E}_{0}}(\xi)$ for all $\xi \in \mathcal{E}_{0}$. Thus, the statement is proved since $D=\left[\pi_{R}(a)\left(1_{A} \otimes \lambda(x) L(y)\right) ; a \in A, x \in \widehat{S}, y \in S\right]$.

7.22 Theorem. - The $\mathcal{G}$-C $C^{*}$-algebras $(A \rtimes \mathcal{G}) \rtimes \widehat{\mathcal{G}}$ and $A$ are Morita equivalent via the $\mathcal{G}$ equivariant imprimitivity $(A \rtimes \mathcal{G}) \rtimes \widehat{\mathcal{G}}$ - $A$-bimodule $\mathcal{E}_{A, R}$.

Proof. Let us prove that the Hilbert $A$-module $\mathcal{E}_{A, R}$ is full. Fix $x \in A$ and write $x=a^{*} b$ for $a, b \in A$. There exists $\omega \in \mathcal{B}(\mathscr{H})_{*}$ such that $\left(\operatorname{id}_{A} \otimes \omega\right)\left(q_{\beta_{A} \widehat{\alpha}}\right)=1_{A}$. Hence, $x$ is the norm limit of finite sums of elements of the form $a^{*}\left(\operatorname{id}_{A} \otimes \omega_{\xi, \eta}\right)\left(q_{\beta_{A} \hat{\alpha}}\right) b$, where $\xi, \eta \in \mathscr{H}$. However, for all $\xi, \eta \in \mathscr{H}$ we have

$$
a^{*}\left(\operatorname{id}_{A} \otimes \omega_{\zeta, \eta}\right)\left(q_{\beta_{A} \widehat{\alpha}}\right) b=(a \otimes \xi)^{*} q_{\beta_{A} \widehat{\alpha}}(b \otimes \eta)=\left\langle q_{\beta_{A^{\widehat{\alpha}}}}(a \otimes \xi), q_{\beta_{A} \widehat{\alpha}}(b \otimes \eta)\right\rangle .
$$

Hence, $A=\left[\langle\xi, \eta\rangle ; \xi, \eta \in \mathcal{E}_{A, R}\right]$. Now, we recall that $D=q_{\beta_{A} \widehat{\alpha}}(A \otimes \mathcal{K}) q_{\beta_{A} \widehat{\alpha}}$ (cf. 5.1.24). It is easily seen that the left action of $(A \rtimes \mathcal{G}) \rtimes \widehat{\mathcal{G}}$ (cf. 7.21) induces a $\mathcal{G}$-equivariant *-isomorphism $(A \rtimes \mathcal{G}) \rtimes \widehat{\mathcal{G}} \simeq \mathcal{K}\left(\mathcal{E}_{A, R}\right)$. 


\section{APPENDIX}

8.1 Normal linear forms, weights and operator-valued weights on von Neumann algebras [8]

Let $M$ be a von Neumann algebra. Denote by $M_{*}\left(\right.$ resp. $\left.M_{*}^{+}\right)$the Banach space (resp. positive cone) of the normal linear forms (resp. positive normal linear forms) on $M$. Let $\omega \in M_{*}$ and $a, b \in M$. Denote by $a \omega \in M_{*}$ and $\omega b \in M_{*}$ the normal linear functionals on $M$ given for all $x \in M$ by:

$$
(a \omega)(x):=\omega(x a) ; \quad(\omega b)(x):=\omega(b x) .
$$

We have $a^{\prime}(a \omega)=\left(a^{\prime} a\right) \omega$ and $(\omega b) b^{\prime}=\omega\left(b b^{\prime}\right)$, for all $a, a, b, b^{\prime} \in M$. We also denote

$$
a \omega b:=a(\omega b)=(a \omega) b ; \quad \omega_{a}:=a^{*} \omega a .
$$

If $\omega \in M_{*}^{+}$, then $\omega_{a} \in M_{*}^{+}$. Note that $\left(\omega_{a}\right)_{b}=\omega_{a b}$ for all $a, b \in M$. If $\omega \in M_{*}$ we define $\bar{\omega} \in M_{*}$ by setting

$$
\bar{\omega}(x):=\overline{\omega\left(x^{*}\right)}, \quad \text { for all } x \in M \text {. }
$$

Let $\mathscr{H}$ be a Hilbert space and let us fix $\xi, \eta \in \mathscr{H}$. Denote by $\omega_{\xi, \eta} \in \mathcal{B}(\mathscr{H})_{*}$ the normal linear form defined by

$$
\omega_{\xi, \eta}(x):=\langle\xi, x \eta\rangle, \text { for all } x \in \mathcal{B}(\mathscr{H}) .
$$

Note that we have $\bar{\omega}_{\xi, \eta}=\omega_{\eta, \xi}$, a $\omega_{\xi, \eta}=\omega_{\xi, a \eta}$ and $\omega_{\xi, \eta} a=\omega_{a^{*} \xi, \eta}$ for all $a \in \mathcal{B}(\mathscr{H})$.

8.1.1. Tensor product of normal linear forms. Let $M$ and $N$ be von Neumann algebras, $\phi \in M_{*}$ and $\psi \in N_{*}$. There exists a unique $\phi \otimes \psi \in(M \otimes N)_{*}$ such that $(\phi \otimes \psi)(x \otimes y)=\phi(x) \psi(y)$ for all $x \in M$ and $y \in N$. Moreover, $\|\phi \otimes \psi\| \leqslant\|\phi\| \cdot\|\psi\|$. Actually, it is known that we have an (completely) isometric identification $M_{*} \widehat{\otimes}_{\pi} N_{*}=(M \otimes N)_{*}$, where $\widehat{\otimes}_{\pi}$ denotes the projective tensor product of Banach spaces. In particular, any $\omega \in(M \otimes N)_{*}$ is the norm limit of finite sums of the form $\sum_{i} \phi_{i} \otimes \psi_{i}$, where $\phi_{i} \in M_{*}$ and $\psi_{i} \in N_{*}$.

8.1.2. Slicing with normal linear forms. We will also need to slice maps with normal linear forms. Let $M_{1}$ and $M_{2}$ be von Neumann algebras, $\omega_{1} \in\left(M_{1}\right)_{*}$ and $\omega_{2} \in\left(M_{2}\right)_{*}$. Therefore, the maps $\omega_{1} \odot$ id $: M_{1} \odot M_{2} \rightarrow M_{1}$ and id $\odot \omega_{2}: M_{1} \odot M_{2} \rightarrow M_{2}$ extend uniquely to norm continuous normal linear maps $\omega_{1} \otimes$ id $: M_{1} \otimes M_{2} \rightarrow M_{2}$ and id $\otimes \omega_{2}: M_{1} \otimes M_{2} \rightarrow M_{1}$. Let $\mathscr{H}$ and $\mathscr{K}$ be Hilbert spaces, for $\xi \in \mathscr{H}$ and $\eta \in \mathscr{K}$ we define $\theta_{\xi} \in \mathcal{B}(\mathscr{K}, \mathscr{H} \otimes \mathscr{K})$ and $\theta_{\eta}^{\prime} \in \mathcal{B}(\mathscr{H}, \mathscr{H} \otimes \mathscr{K})$ by setting:

$$
\theta_{\xi}(\zeta):=\xi \otimes \zeta, \quad \text { for all } \zeta \in \mathscr{K} ; \quad \theta_{\eta}^{\prime}(\zeta):=\zeta \otimes \eta, \quad \text { for all } \zeta \in \mathscr{H} .
$$

If $T \in \mathcal{B}(\mathscr{H} \otimes \mathscr{K}), \phi \in \mathcal{B}(\mathscr{K})_{*}$ and $\omega \in \mathcal{B}(\mathscr{H})_{*}$, then the operators $(\mathrm{id} \otimes \phi)(T) \in \mathcal{B}(\mathscr{H})$ and $(\omega \otimes \mathrm{id})(T) \in \mathcal{B}(\mathscr{K})$ are determined by the formulas:

$$
\begin{aligned}
& \left\langle\xi_{1},(\mathrm{id} \otimes \phi)(T) \xi_{2}\right\rangle=\phi\left(\theta_{\xi_{1}}^{*} T \theta_{\xi_{2}}\right), \quad \xi_{1}, \xi_{2} \in \mathscr{H} ; \\
& \left\langle\eta_{1},(\omega \otimes \mathrm{id})(T) \eta_{2}\right\rangle=\omega\left(\theta_{\eta_{1}}^{*} T \theta_{\eta_{2}}^{\prime}\right), \quad \eta_{1}, \eta_{2} \in \mathscr{K} .
\end{aligned}
$$

In particular, we have:

$$
\left(\mathrm{id} \otimes \omega_{\eta_{1}, \eta_{2}}\right)(T)=\theta_{\eta_{1}}^{* *} T \theta_{\eta_{2}}^{\prime}, \quad \eta_{1}, \eta_{2} \in \mathscr{K} ; \quad\left(\omega_{\xi_{1}, \xi_{2}} \otimes \mathrm{id}\right)(T)=\theta_{\xi_{1}}^{*} T \theta_{\xi_{2}}, \quad \xi_{1}, \xi_{2} \in \mathscr{H} .
$$

Let us recall some formulas that will be used several times. For all $\phi \in \mathcal{B}(\mathscr{K})_{*}, \omega \in \mathcal{B}(\mathscr{H})_{*}$ and $T \in \mathcal{B}(\mathscr{H} \otimes \mathscr{K})$, we have:

$$
\begin{aligned}
& x(\mathrm{id} \otimes \phi)(T) y=(\mathrm{id} \otimes \phi)((x \otimes 1) T(y \otimes 1)),(y \omega x \otimes \mathrm{id})(T)=(\omega \otimes \mathrm{id})((x \otimes 1) T(y \otimes 1)) \\
& \text { for all } x, y \in \mathcal{B}(\mathscr{H}) ; \\
& \quad a(\omega \otimes \mathrm{id})(T) b=(\omega \otimes \mathrm{id})((1 \otimes a) T(1 \otimes b)),(\mathrm{id} \otimes b \phi a)(T)=(\mathrm{id} \otimes \phi)((1 \otimes a) T(1 \otimes b))
\end{aligned}
$$

for all $a, b \in \mathcal{B}(\mathscr{K})$. We also have

$$
\begin{array}{cl}
(\mathrm{id} \otimes \phi)(T)^{*}=(\mathrm{id} \otimes \bar{\phi})\left(T^{*}\right), & (\omega \otimes \mathrm{id})(T)^{*}=(\bar{\omega} \otimes \mathrm{id})\left(T^{*}\right), \\
(\phi \otimes \mathrm{id})\left(\Sigma_{\mathscr{H} \otimes \mathscr{K}} T \Sigma_{\mathscr{K} \otimes \mathscr{H}}\right)=(\mathrm{id} \otimes \phi)(T), & (\mathrm{id} \otimes \omega)\left(\Sigma_{\mathscr{H} \otimes \mathscr{K}} T \Sigma_{\mathscr{K} \otimes \mathscr{H}}\right)=(\omega \otimes \mathrm{id})(T),
\end{array}
$$

for all $T \in \mathcal{B}(\mathscr{H} \otimes \mathscr{K}), \phi \in \mathcal{B}(\mathscr{K})_{*}$ and $\omega \in \mathcal{B}(\mathscr{H})_{*}$. 
8.1.3 Definition. - A weight $\varphi$ on $M$ is a map $\varphi: M_{+} \rightarrow[0, \infty]$ such that:

- for all $x, y \in M_{+}, \varphi(x+y)=\varphi(x)+\varphi(y)$;

- for all $x \in M_{+}$and $\lambda \in \mathbb{R}_{+}, \varphi(\lambda x)=\lambda \varphi(x)$.

We denote by $\mathfrak{N}_{\varphi}:=\left\{x \in M ; \varphi\left(x^{*} x\right)<\infty\right\}$ the left ideal of square $\varphi$-integrable elements of $M, \mathfrak{M}_{\varphi}^{+}:=\left\{x \in M_{+} ; \varphi(x)<\infty\right\}$ the cone of positive $\varphi$-integrable elements of $M$ and $\mathfrak{M}_{\varphi}:=\left\langle\mathfrak{M}_{\varphi}^{+}\right\rangle$the space of $\varphi$-integrable elements of $M$.

8.1.4 Definition. - Let $\varphi$ be a weight on $M$. The opposite weight of $\varphi$ is the weight $\varphi^{\mathrm{o}}$ on $M^{\mathrm{o}}$ given by $\varphi^{\mathrm{o}}\left(x^{\mathrm{o}}\right):=\varphi(x)$ for all $x \in M_{+}$. Then, we have $\mathfrak{N}_{\varphi^{\mathrm{o}}}=\left(\mathfrak{N}_{\varphi^{*}}^{*}\right)^{\mathrm{o}}, \mathfrak{M}_{\varphi^{\mathrm{o}}}^{+}=\left(\mathfrak{M}_{\varphi}^{+}\right)^{\mathrm{o}}$ and $\mathfrak{M}_{\varphi^{\circ}}=\left(\mathfrak{M}_{\varphi}\right)^{\mathrm{o}}$.

8.1.5 Definition. - A weight $\varphi$ on $M$ is called:

- semi-finite, if $\mathfrak{N}_{\varphi}$ is $\sigma$-weakly dense in $M$;

- faithful, if for $x \in M_{+}$the condition $\varphi(x)=0$ implies $x=0$;

- normal, if $\varphi\left(\sup _{i \in \mathcal{I}} x_{i}\right)=\sup _{i \in \mathcal{I}} \varphi\left(x_{i}\right)$ for all increasing bounded net $\left(x_{i}\right)_{i \in \mathcal{I}}$ of $M_{+}$.

From now on, we will mainly use normal semi-finite faithful (n.s.f.) weights. Fix a n.s.f. weight $\varphi$ on $M$.

8.1.6 Definition. - We define an inner product on $\mathfrak{N}_{\varphi}$ by setting

$$
\langle x, y\rangle_{\varphi}:=\varphi\left(x^{*} y\right), \quad \text { for all } x, y \in \mathfrak{N}_{\varphi} .
$$

We denote by $\left(\mathscr{H}_{\varphi}, \Lambda_{\varphi}\right)$ the Hilbert space completion of $\mathfrak{N}_{\varphi}$ with respect to this inner product, where $\Lambda_{\varphi}: \mathfrak{N}_{\varphi} \rightarrow \mathscr{H}_{\varphi}$ is the canonical map. There exists a unique unital normal *-representation $\pi_{\varphi}: M \rightarrow \mathcal{B}\left(\mathscr{H}_{\varphi}\right)$ such that

$$
\pi_{\varphi}(x) \Lambda_{\varphi}(y)=\Lambda_{\varphi}(x y), \quad \text { for all } x \in M \text { and } y \in \mathfrak{N}_{\varphi} .
$$

The triple $\left(\mathscr{H}_{\varphi}, \pi_{\varphi}, \Lambda_{\varphi}\right)$ is called the G.N.S. construction for $(M, \varphi)$.

8.1.7 Remarks. - The linear map $\Lambda_{\varphi}$ is called the G.N.S. map. We have that $\Lambda_{\varphi}\left(\mathfrak{N}_{\varphi}\right)$ is dense in $\mathscr{H}_{\varphi}$ and $\left\langle\Lambda_{\varphi}(x), \Lambda_{\varphi}(y)\right\rangle_{\varphi}=\varphi\left(x^{*} y\right)$ for all $x, y \in \mathfrak{N}_{\varphi}$. In particular, $\Lambda_{\varphi}$ is injective. Moreover, we also call $\pi_{\varphi}$ the G.N.S. representation.

We recall below the main objects of the Tomita-Takesaki modular theory.

8.1.8 Proposition-Definition. - Let $M$ be a von Neumann algebra and $\varphi$ a n.s.f. weight on $M$. The anti-linear map $\Lambda_{\varphi}\left(\mathfrak{N}_{\varphi}^{*} \cap \mathfrak{N}_{\varphi}\right) \rightarrow \Lambda_{\varphi}\left(\mathfrak{N}_{\varphi}^{*} \cap \mathfrak{N}_{\varphi}\right) ; \Lambda_{\varphi}(x) \mapsto \Lambda_{\varphi}\left(x^{*}\right)$ is closable and its closure is a possibly unbounded anti-linear map $\mathcal{T}_{\varphi}: D\left(\mathcal{T}_{\varphi}\right) \subset \mathscr{H}_{\varphi} \rightarrow \mathscr{H}_{\varphi}$ such that $D\left(\mathcal{T}_{\varphi}\right)=\operatorname{im} \mathcal{T}_{\varphi}$ and $\mathcal{T}_{\varphi} \circ \mathcal{T}_{\varphi}(x)=x$ for all $x \in D\left(\mathcal{T}_{\varphi}\right)$.

Let $\mathcal{T}_{\varphi}=J_{\varphi} \nabla_{\varphi}^{1 / 2}$ be the polar decomposition of $\mathcal{T}_{\varphi}$. The anti-unitary $J_{\varphi}: \mathscr{H}_{\varphi} \rightarrow \mathscr{H}_{\varphi}$ is called the modular conjugation for $\varphi$ and the injective positive self-adjoint operator $\nabla_{\varphi}$ is called the modular operator for $\varphi$.

8.1.9 Proposition-Definition. - There exists a unique one-parameter group $\left(\sigma_{t}^{\varphi}\right)_{t \in \mathbb{R}}$ of automorphisms on $M$, called the modular automorphism group of $\varphi$, such that

$$
\pi_{\varphi}\left(\sigma_{t}^{\varphi}(x)\right)=\nabla_{\varphi}^{\mathrm{i} t} \pi_{\varphi}(x) \nabla_{\varphi}^{-\mathrm{i} t}, \quad \text { for all } t \in \mathbb{R} \text { and } x \in M .
$$

Then, for all $t \in \mathbb{R}$ and $x \in M$ we have $\sigma_{t}^{\varphi}(x) \in \mathfrak{N}_{\varphi}$ and $\Lambda_{\varphi}\left(\sigma_{t}^{\varphi}(x)\right)=\nabla_{\varphi}^{\mathrm{it}} \Lambda_{\varphi}(x)$.

8.1.1o Proposition-Definition. - The map $C_{M}: M \rightarrow M^{\prime} ; x \mapsto J_{\varphi} \pi_{\varphi}(x)^{*} J_{\varphi}$ is a normal unital *-antihomomorphism.

8.1.11 Definition. - Let $N$ be a von Neumann algebra. The extended positive cone of $N$ is the set $N_{+}^{\text {ext }}$ consisting of the maps $m: N_{*}^{+} \rightarrow[0, \infty]$, which satisfy the following conditions: 
- for all $\omega_{1}, \omega_{2} \in N_{*}^{+}, m\left(\omega_{1}+\omega_{2}\right)=m\left(\omega_{1}\right)+m\left(\omega_{2}\right)$;

- for all $\omega \in N_{*}^{+}$and $\lambda \in \mathbb{R}_{+}, m(\lambda \omega)=\lambda m(\omega)$;

- $m$ is lower semicontinuous with respect to the norm topology on $N_{*}$.

8.1.12 Notations. - Let $N$ be a von Neumann algebra.

1. From now on, we will identify $N_{+}$with its part inside $N_{+}^{\text {ext }}$. Accordingly, if $m \in N_{+}^{\text {ext }}$ and $\omega \in N_{*}^{+}$we will denote by $\omega(m)$ the evaluation of $m$ at $\omega$.

2. Let $a \in N$ and $m \in N_{+}^{\text {ext }}$, we define $a^{*} m a \in N_{+}^{\text {ext }}$ by setting $\omega\left(a^{*} m a\right):=a \omega a^{*}(m)$ for all $\omega \in N_{*}^{+}$. If $m, n \in N_{+}^{\text {ext }}$ and $\lambda \in \mathbb{R}_{+}$, we also define $m+n \in N_{+}^{\text {ext }}$ and $\lambda m \in N_{+}^{\text {ext }}$ by setting $\omega(m+n):=\omega(m)+\omega(n)$ and $\omega(\lambda m):=\lambda \omega(m)$ for all $\omega \in N_{*}^{+}$.

8.1.13 Definition. - Let $N \subset M$ be a unital normal inclusion of von Neumann algebras. An operator-valued weight from $M$ to $N$ is a map $T: M_{+} \rightarrow N_{+}^{\text {ext }}$ such that:

- for all $x, y \in M_{+}, T(x+y)=T(x)+T(y)$;

- for all $x \in M_{+}, \forall \lambda \in \mathbb{R}_{+}, T(\lambda x)=\lambda T(x)$;

- for all $x \in M_{+}$and $a \in N, T\left(a^{*} x a\right)=a^{*} T(x) a$.

Let $\mathfrak{N}_{T}:=\left\{x \in M ; T\left(x^{*} x\right) \in N_{+}\right\}, \mathfrak{M}_{T}^{+}:=\left\{x \in M_{+} ; T(x) \in N_{+}\right\}$and $\mathfrak{M}_{T}:=\left\langle\mathfrak{M}_{T}^{+}\right\rangle$.

8.1.14 Definition. - Let $N \subset M$ be a unital normal inclusion of von Neumann algebras. An operator-valued weight $T$ from $M$ to $N$ is said to be:

- semi-finite, if $\mathfrak{N}_{T}$ is $\sigma$-weakly dense in $M$;

- faithful, if for $x \in M_{+}$the condition $T(x)=0$ implies $x=0$;

- normal, if for every increasing bounded net $\left(x_{i}\right)_{i \in \mathcal{I}}$ of elements of $M_{+}$and $\omega \in N_{*}^{+}$, we have $\omega\left(T\left(\sup _{i \in \mathcal{I}} x_{i}\right)\right)=\lim _{i \in \mathcal{I}} \omega\left(T\left(x_{i}\right)\right)$.

Note that if $T: M_{+} \rightarrow N_{+}^{\text {ext }}$ is an operator-valued weight, it extends uniquely to a semilinear map $\bar{T}: M_{+}^{\text {ext }} \rightarrow N_{+}^{\text {ext }}$. This will allow us to compose n.s.f. operator-valued weights. Indeed, let $P \subset N \subset M$ be unital normal inclusions of von Neumann algebras. Let $S$ (resp. T) be an operator-valued weight from $N$ (resp. $M)$ to $P$ (resp. $N)$. We define an operator-valued weight from $M$ to $P$ by setting $(S \circ T)(x):=\bar{S}(T(x))$ for all $x \in N_{+}$.

\subsection{Relative tensor product of Hilbert spaces and fiber product of von Neumann algebras}

In this paragraph, we will recall the definitions, notations and important results concerning the relative tensor product and the fiber product which are the main technical tools of the theory of measured quantum groupoids. For more information, we refer the reader to [7].

In the whole section, $N$ is a von Neumann algebra endowed with a n.s.f. weight $\varphi$. Let $\pi$ (resp. $\gamma$ ) be a normal unital ${ }^{*}$-representation of $N\left(\right.$ resp. $N^{\circ}$ ) on a Hilbert space $\mathcal{H}$ (resp. $\mathcal{K}$ ).

Relative tensor product. The Hilbert space $\mathcal{H}$ (resp. $\mathcal{K}$ ) may be considered as a left (resp. right) $N$-module. Moreover, $\mathscr{H}_{\varphi}$ is an $N$-bimodule whose actions are given by

$$
x \xi:=\pi_{\varphi}(x) \xi \quad \text { and } \quad \xi y:=J_{\varphi} \pi_{\varphi}\left(y^{*}\right) J_{\varphi} \xi, \quad \text { for all } \xi \in \mathscr{H}_{\varphi} \text { and } x, y \in N .
$$

8.2.1 Definition. - We define the set of right (resp. left) bounded vectors with respect to $\varphi$ and $\pi$ (resp. $\gamma$ ) to be:

$$
\begin{gathered}
\varphi(\pi, \mathcal{H}):=\left\{\xi \in \mathcal{H} ; \exists C \in \mathbb{R}_{+}, \forall x \in \mathfrak{N}_{\varphi},\|\pi(x) \xi\| \leqslant C\left\|\Lambda_{\varphi}(x)\right\|\right\}, \\
\text { (resp. } \left.(\mathcal{K}, \gamma)_{\varphi}:=\left\{\xi \in \mathcal{K} ; \exists C \in \mathbb{R}_{+}, \forall x \in \mathfrak{N}_{\varphi}^{*},\left\|\gamma\left(x^{\mathrm{o}}\right) \xi\right\| \leqslant C\left\|\Lambda_{\varphi^{\circ}}\left(x^{\mathrm{o}}\right)\right\|\right\}\right) .
\end{gathered}
$$


If $\xi \in{ }_{\varphi}(\pi, \mathcal{H})$, we denote by $R_{\tilde{\xi}}^{\pi, \varphi} \in \mathcal{B}\left(\mathscr{H}_{\varphi}, \mathcal{H}\right)$ (or simply $R_{\tilde{\zeta}}^{\pi}$ if $\varphi$ is understood) the unique bounded operator such that

$$
R_{\xi}^{\pi, \varphi} \Lambda_{\varphi}(x)=\pi(x) \xi, \quad \text { for all } x \in \mathfrak{N}_{\varphi} .
$$

Similarly, if $\xi \in(\mathcal{K}, \gamma)_{\varphi}$ we denote $L_{\xi}^{\gamma, \varphi} \in \mathcal{B}\left(\mathscr{H}_{\varphi}, \mathcal{K}\right)$ (or simply $L_{\xi}^{\gamma}$ if $\varphi$ is understood) the unique bounded operator such that

$$
L_{\xi}^{\gamma, \varphi} J_{\varphi} \Lambda_{\varphi}\left(x^{*}\right)=\gamma\left(x^{\mathrm{o}}\right) \xi, \quad \text { for all } x \in \mathfrak{N}_{\varphi}^{*},
$$

where we have used the identification $\mathscr{H}_{\varphi^{\mathrm{o}}} \rightarrow \mathscr{H}_{\varphi} ; \Lambda_{\varphi^{\mathrm{o}}}\left(x^{\mathrm{o}}\right) \mapsto J_{\varphi} \Lambda_{\varphi}\left(x^{*}\right)$.

Note that $\xi \in \mathcal{K}$ is left bounded with respect to $\varphi$ and $\gamma$ if, and only if, it is right bounded with respect to the n.s.f. weight $\varphi^{\mathrm{c}}:=\varphi \circ C_{N}^{-1}$ on $N^{\prime}$ and the normal unital ${ }^{*}$-representation $\gamma^{\mathrm{c}}:=\gamma \circ C_{N}^{-1}$ of $N^{\prime}$. It is important to note that $(\mathcal{K}, \gamma)_{\varphi}(\operatorname{resp} . \varphi(\pi, \mathcal{H}))$ is dense in $\mathcal{K}$ (resp. $\mathcal{H})$ (cf. Lemma 2 of $[7]$ ).

If $\xi \in{ }_{\varphi}(\pi, \mathcal{H})$ (resp. $\xi \in(\mathcal{K}, \gamma)_{\varphi}$ ), we have that $R_{\xi}^{\pi, \varphi}$ (resp. $\left.L_{\xi}^{\gamma, \varphi}\right)$ is left (resp. right) $N$-linear. Therefore, for all $\xi, \eta \in \in_{\varphi}(\pi, \mathcal{H})$ (resp. $\left.(\mathcal{K}, \gamma)_{\varphi}\right)$ we have

$$
\begin{gathered}
\left(R_{\mathcal{\xi}}^{\pi, \varphi}\right)^{*} R_{\eta}^{\pi, \varphi} \in \pi_{\varphi}(N)^{\prime}=C_{N}(N) \text { and } R_{\mathcal{\zeta}}^{\pi, \varphi}\left(R_{\eta}^{\pi, \varphi}\right)^{*} \in \pi(N)^{\prime} \\
\text { (resp. } \left.\left(L_{\xi}^{\gamma, \varphi}\right)^{*} L_{\eta}^{\gamma, \varphi} \in \pi_{\varphi}(N) \text { and } L_{\xi}^{\gamma, \varphi}\left(L_{\eta}^{\gamma, \varphi}\right)^{*} \in \gamma\left(N^{\mathrm{o}}\right)^{\prime}\right) .
\end{gathered}
$$

8.2.2 Notations. - (cf. 2.1 [16]) Let

$$
\left.\mathcal{K}_{\pi, \varphi}:=\left[R_{\xi}^{\pi, \varphi}\left(R_{\eta}^{\pi, \varphi}\right)^{*} ; \xi, \eta \in \in_{\varphi}(\pi, \mathcal{H})\right] \quad \text { (resp. } \mathcal{K}_{\gamma, \varphi}:=\left[L_{\xi}^{\gamma, \varphi}\left(L_{\eta}^{\gamma, \varphi}\right)^{*} ; \xi, \eta \in(\mathcal{H}, \gamma)_{\varphi}\right]\right) .
$$

Note that $\mathcal{K}_{\pi, \varphi}$ (resp. $\mathcal{K}_{\gamma, \varphi}$ ) is a weakly dense ideal of $\pi(N)^{\prime}$ (resp. $\left.\gamma\left(N^{\circ}\right)^{\prime}\right)$ (cf. Proposition 3 of [7]). If $\varphi$ is understood, we denote $\mathcal{K}_{\pi}$ (resp. $\mathcal{K}_{\gamma}$ ) instead of $\mathcal{K}_{\pi, \varphi}$ (resp. $\mathcal{K}_{\gamma, \varphi}$ ).

8.2.3 Notations. - Let $\xi, \eta \in{ }_{\varphi}(\pi, \mathcal{H})\left(\operatorname{resp} .(\mathcal{K}, \gamma)_{\varphi}\right)$, we denote

$$
\langle\xi, \eta\rangle_{N^{\circ}}:=C_{N}^{-1}\left(\left(R_{\xi}^{\pi, \varphi}\right)^{*} R_{\eta}^{\pi, \varphi}\right)^{\mathrm{o}} \in N^{\mathrm{o}} \quad\left(\operatorname{resp} .\langle\xi, \eta\rangle_{N}:=\pi_{\varphi}^{-1}\left(\left(L_{\xi}^{\gamma, \varphi}\right)^{*} L_{\eta}^{\gamma, \varphi}\right) \in N\right) .
$$

8.2.4 Proposition. - For all $\xi, \eta \in{ }_{\varphi}(\pi, \mathcal{H})\left(\right.$ resp. $\left.\xi, \eta \in(\mathcal{K}, \gamma)_{\varphi}\right)$ and $y \in N$ analytic for $\left(\sigma_{t}^{\varphi}\right)_{t \in \mathbb{R}}$, we have:

1. $\langle\xi, \eta\rangle_{N^{\circ}}^{*}=\langle\eta, \xi\rangle_{N^{\circ}}\left(\operatorname{resp} .\langle\xi, \eta\rangle_{N}^{*}=\langle\eta, \xi\rangle_{N}\right) ;$

2. $\left\langle\xi, \eta y^{\mathrm{o}}\right\rangle_{N^{\mathrm{o}}}=\langle\xi, \eta\rangle_{N^{\mathrm{o}}} \sigma_{\mathrm{i} / 2}^{\varphi}(y)^{\mathrm{o}}\left(\right.$ resp. $\left.\langle\xi, \eta y\rangle_{N}=\langle\xi, \eta\rangle_{N} \sigma_{-\mathrm{i} / 2}^{\varphi}(y)\right)$.

8.2.5 Lemma. - For all $\xi_{1}, \xi_{2} \in{ }_{\varphi}(\pi, \mathcal{H})$ and $\eta_{1}, \eta_{2} \in(\mathcal{K}, \gamma)_{\varphi}$, we have

$$
\left\langle\eta_{1}, \gamma\left(\left\langle\xi_{1}, \xi_{2}\right\rangle_{N^{\circ}}\right) \eta_{2}\right\rangle_{\mathcal{K}}=\left\langle\xi_{1}, \pi\left(\left\langle\eta_{1}, \eta_{2}\right\rangle_{N}\right) \xi_{2}\right\rangle_{\mathcal{H}} .
$$

8.2.6 Definition. - The relative tensor product

$$
\mathcal{K}_{\gamma} \otimes_{\varphi} \mathcal{H} \quad \text { (or simply denoted by } \mathcal{K}_{\gamma} \otimes_{\pi} \mathcal{H} \text { ) }
$$

is the Hausdorff completion of the pre-Hilbert space $(\mathcal{K}, \gamma)_{\varphi} \odot{ }_{\varphi}(\pi, \mathcal{H})$, whose inner product is given by

$$
\left\langle\eta_{1} \otimes \xi_{1}, \eta_{2} \otimes \xi_{2}\right\rangle:=\left\langle\eta_{1}, \gamma\left(\left\langle\xi_{1}, \xi_{2}\right\rangle_{N^{\circ}}\right) \eta_{2}\right\rangle_{\mathcal{K}}=\left\langle\xi_{1}, \pi\left(\left\langle\eta_{1}, \eta_{2}\right\rangle_{N}\right) \xi_{2}\right\rangle_{\mathcal{H}},
$$

for all $\eta_{1}, \eta_{2} \in(\mathcal{K}, \gamma)_{\varphi}$ and $\xi_{1}, \xi_{2} \in{ }_{\varphi}(\pi, \mathcal{H})$. If $\eta \in(\mathcal{K}, \gamma)_{\varphi}$ and $\xi \in{ }_{\varphi}(\pi, \mathcal{H})$, we will denote by

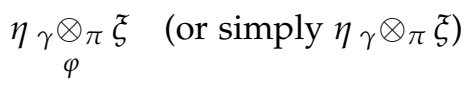

the image of $\eta \otimes \xi$ by the canonical map $(\mathcal{K}, \gamma)_{\varphi} \odot \varphi_{\varphi}(\pi, \mathcal{H}) \rightarrow \mathcal{K}_{\gamma} \otimes_{\pi} \mathcal{H}$ (isometric dense range). 
8.2.7 Remarks. - $\quad$ 1. By applying this construction to $\left(N^{\circ}, \varphi^{\circ}\right)$ instead of $(N, \varphi)$ we obtain the relative tensor product $\mathcal{H}_{\pi} \otimes_{\gamma} \mathcal{K}$.

2. The relative tensor product $\mathcal{K}_{\gamma} \otimes_{\pi} \mathcal{H}$ is also the Hausdorff completion of the preHilbert space $(\mathcal{K}, \gamma)_{\varphi} \odot \mathcal{H}\left(\right.$ resp. $\left.\mathcal{K} \odot \varphi_{\varphi}(\pi, \mathcal{H})\right)$, whose inner product is given by:

$$
\begin{gathered}
\left\langle\eta_{1} \otimes \xi_{1}, \eta_{2} \otimes \xi_{2}\right\rangle:=\left\langle\xi_{1}, \pi\left(\left\langle\eta_{1}, \eta_{2}\right\rangle_{N}\right) \xi_{2}\right\rangle_{\mathcal{H}} \\
\text { (resp. } \left.\left\langle\eta_{1} \otimes \xi_{1}, \eta_{2} \otimes \xi_{2}\right\rangle:=\left\langle\eta_{1}, \gamma\left(\left\langle\xi_{1}, \xi_{2}\right\rangle_{N^{\circ}}\right) \eta_{2}\right\rangle_{\mathcal{K}}\right) .
\end{gathered}
$$

3. Moreover, for all $\eta \in \mathcal{K}, \xi \in{ }_{\varphi}(\pi, \mathcal{H})$ and $y \in N$ analytic for $\left(\sigma_{t}^{\varphi}\right)_{t \in \mathbb{R}}$ we have

$$
\gamma\left(y^{\mathrm{o}}\right) \eta_{\gamma} \otimes_{\pi} \xi=\eta_{\gamma} \otimes_{\pi} \pi\left(\sigma_{-\mathrm{i} / 2}^{\varphi}(y)\right) \xi .
$$

8.2.8. The relative flip map is the isomorphism $\sigma_{\varphi}^{\gamma \pi}$ from $\mathcal{K}_{\gamma} \otimes_{\varphi} \underset{\mathcal{H}}{ }$ onto $\mathcal{H}_{\pi} \otimes_{\varphi^{\circ}} \mathcal{K}$ given by:

$$
\sigma_{\varphi}^{\gamma \pi}\left(\eta_{\gamma} \otimes_{\varphi} \xi\right):=\xi_{\varphi^{\circ}}^{\otimes_{\gamma}} \eta, \quad \text { for all } \xi \in(\mathcal{K}, \gamma)_{\varphi} \text { and } \eta \in \varphi_{\varphi}(\pi, \mathcal{H}) \quad \text { (or simply } \sigma_{\gamma \pi} \text { ). }
$$

Note that $\sigma_{\varphi}^{\gamma \pi}$ is unitary and $\left(\sigma_{\varphi}^{\gamma \pi}\right)^{*}=\sigma_{\varphi^{0}}^{\pi \gamma}$. Then, we can define a relative flip *homomorphism

$$
\left.\varsigma_{\varphi}^{\gamma \pi}: \mathcal{B}\left(\mathcal{K}_{\gamma} \otimes_{\varphi} \mathcal{H}\right) \rightarrow \mathcal{B}\left(\mathcal{H}_{\pi} \otimes_{\varphi^{\circ}} \mathcal{K}\right) \quad \text { (or simply denoted by } \varsigma_{\gamma \pi}\right)
$$

by setting $\varsigma_{\varphi}^{\gamma \pi}(X):=\sigma_{\varphi}^{\gamma \pi} X\left(\sigma_{\varphi}^{\gamma \pi}\right)^{*}$ for all $X \in \mathcal{B}\left(\mathcal{K}_{\gamma} \otimes_{\varphi} \mathcal{H}\right)$.

FIBER PRODUCT OF VON NEUMANN ALGEBRAS. We continue to use the notations of the previous paragraph.

8.2.9 Proposition-Definition. - Let $\mathcal{K}_{i}$ and $\mathcal{H}_{i}$ be Hilbert spaces, and $\gamma_{i}: N^{0} \rightarrow \mathcal{B}\left(\mathcal{K}_{i}\right)$ and $\pi_{i}: N \rightarrow \mathcal{B}\left(\mathcal{H}_{i}\right)$ be unital normal ${ }^{*}$-homomorphisms for $i=1$,2. Let $T \in \mathcal{B}\left(\mathcal{K}_{1}, \mathcal{K}_{2}\right)$ and $S \in \mathcal{B}\left(\mathcal{H}_{1}, \mathcal{H}_{2}\right)$ such that $T \circ \gamma_{1}\left(n^{\circ}\right)=\gamma_{2}\left(n^{\circ}\right) \circ T$ and $S \circ \pi_{1}(n)=\pi_{2}(n) \circ S$ for all $n \in N$. Then, the linear map

$$
\left(\mathcal{K}_{1}, \gamma_{1}\right)_{\varphi} \odot{ }_{\varphi}\left(\pi_{1}, \mathcal{H}_{1}\right) \rightarrow \mathcal{K}_{2 \gamma_{2}} \otimes_{\pi_{2}} \mathcal{H}_{2} ; \xi \odot \eta \mapsto T \xi_{\gamma_{2}} \otimes_{\pi_{2}} S \eta
$$

extends uniquely to a bounded operator $\gamma_{2} T_{\gamma_{1}} \otimes_{\pi_{2}} S_{\pi_{1}} \in \mathcal{B}\left(\mathcal{K}_{1 \gamma_{1}} \otimes_{\pi_{1}} \mathcal{H}_{1}, \mathcal{K}_{2 \gamma_{2}} \otimes_{\pi_{2}} \mathcal{H}_{2}\right)$ (or simply denoted by $T \gamma_{1} \otimes_{\pi_{2}} S$ ), whose adjoint operator is $\gamma_{1} T^{*} \gamma_{2} \otimes_{\pi_{1}} S^{*} \pi_{2}$ (or simply $T^{*} \gamma_{2} \otimes_{\pi_{1}} S^{*}$ ). In particular, if $x \in \gamma\left(N^{\circ}\right)^{\prime}$ and $y \in \pi(N)^{\prime}$, then the linear map

$$
(\mathcal{K}, \gamma)_{\varphi} \odot{ }_{\varphi}(\pi, \mathcal{H}) \rightarrow \mathcal{K}_{\gamma} \otimes_{\pi} \mathcal{H} ; \xi \odot \eta \mapsto x \xi_{\gamma} \otimes_{\pi} y \eta
$$

extends uniquely to a bounded operator on $\mathcal{K}_{\gamma} \otimes_{\pi} \mathcal{H}$ denoted by $x_{\gamma} \otimes_{\pi} y \in \mathcal{B}\left(\mathcal{K}_{\gamma} \otimes_{\pi} \mathcal{H}\right)$.

8.2.10 RemARK. - With the notations of 8.2.9 let $T: \mathcal{K}_{1} \rightarrow \mathcal{H}_{2}$ and $S: \mathcal{H}_{1} \rightarrow \mathcal{K}_{2}$ be bounded antilinear maps such that $T \circ \gamma_{1}\left(n^{\circ}\right)^{*}=\pi_{2}(n) \circ T$ and $S \circ \pi_{1}(n)=\gamma_{2}\left(n^{\circ}\right)^{*} \circ S$ for all $n \in N$. In a similar way, we define $\pi_{2} T_{\gamma_{1}} \otimes_{\gamma_{2}} S_{\pi_{1}} \in \mathcal{B}\left(\mathcal{K}_{1 \gamma_{1}} \otimes_{\pi_{1}} \mathcal{H}_{1}, \mathcal{H}_{2} \pi_{2} \otimes_{\gamma_{2}} \mathcal{K}_{2}\right)$ (or simply $T \gamma_{\gamma_{1}} \otimes_{\gamma_{2}} S$ ). Note that these notations are different from those used in [17, 20].

Let $M \subset \mathcal{B}(\mathcal{K})$ and $P \subset \mathcal{B}(\mathcal{H})$ be two von Neumann algebras. Let us assume that $\pi(N) \subset P$ and $\gamma\left(N^{\mathrm{o}}\right) \subset M$.

8.2.11 Definition. - The fiber product $M_{\gamma^{\star} \star_{\pi}} P$ of $M$ and $P$ over $N$ is the commutant of $\left\{x_{\gamma} \otimes_{\pi} y ; x \in M^{\prime}, y \in P^{\prime}\right\} \subset \mathcal{B}\left(\mathcal{K}_{\gamma} \otimes_{\pi} \mathcal{H}\right)$. Then, $M_{\gamma^{\star} \pi} P$ is a von Neumann algebra.

Note that we have $\varsigma_{\gamma \pi}\left(M_{\gamma^{\star} \pi} P\right)=P_{\pi^{\star} \gamma} M$. We still denote by $\varsigma_{\gamma \pi}: M_{\gamma^{\star} \pi} P \rightarrow P_{\pi^{\star} \gamma} M$ the restriction of $\varsigma_{\gamma \pi}$ to $M_{\gamma \star}{ }^{\star} P$. 
8.2.12. Slicing with normal linear forms. Now, let us recall how to slice with normal linear forms. For $\xi \in(\mathcal{K}, \gamma)_{\varphi}$ and $\eta \in \in_{\varphi}(\pi, \mathcal{H})$, we consider the following bounded linear maps:

$$
\lambda_{\tilde{\zeta}}^{\gamma \pi}: \mathcal{H} \rightarrow \mathcal{K}_{\gamma} \otimes_{\pi} \mathcal{H}, \zeta \mapsto \xi_{\gamma} \otimes_{\pi} \zeta ; \quad \rho_{\eta}^{\gamma \pi}: \mathcal{K} \rightarrow \mathcal{K}_{\gamma} \otimes_{\pi} \mathcal{H}, \zeta \mapsto \zeta_{\gamma} \otimes_{\pi} \eta .
$$

Let $T \in \mathcal{B}\left(\mathcal{K}_{\gamma} \otimes_{\pi} \mathcal{H}\right)$ and $\omega \in \mathcal{B}(\mathcal{H})_{*}\left(\right.$ resp. $\left.\omega \in \mathcal{B}(\mathcal{K})_{*}\right)$. By using the fact that $(\mathcal{K}, \gamma)_{\varphi}$ (resp. $\varphi(\pi, \mathcal{H})$ ) is dense in $\mathcal{H}$ (resp. $\mathcal{K})$, there exists a unique $\left(\right.$ id $\left._{\gamma^{\star} \pi} \omega\right)(T) \in \mathcal{B}(\mathcal{K})$ (resp. $\left.\left(\omega_{\gamma \star} \pi \mathrm{id}\right)(T) \in \mathcal{B}(\mathcal{H})\right)$ such that

$$
\begin{array}{rlrl}
\left\langle\xi_{1},\left(\operatorname{id}_{\gamma^{\star} \pi} \omega\right)(T) \xi_{2}\right\rangle & =\omega\left(\left(\lambda_{\xi_{1}}^{\gamma \pi}\right)^{*} T \lambda_{\xi_{2}}^{\gamma \pi}\right), & \text { for all } \xi_{1}, \xi_{2} \in(\mathcal{K}, \gamma)_{\varphi} \\
\text { (resp. }\left\langle\eta_{1},\left(\omega_{\gamma^{\star} \pi} \mathrm{id}\right)(T) \eta_{2}\right\rangle & =\omega\left(\left(\rho_{\eta_{1}}^{\gamma \pi}\right)^{*} T \rho_{\eta_{2}}^{\gamma \pi}\right), & & \text { for all } \left.\eta_{1}, \eta_{2} \in \varphi(\pi, \mathcal{H})\right) .
\end{array}
$$

In particular, we have:

$$
\begin{aligned}
& \left(\operatorname{id}_{\gamma^{\star} \pi} \omega_{\eta_{1}, \eta_{2}}\right)(T)=\left(\rho_{\eta_{1}}^{\gamma \pi}\right)^{*} T \rho_{\eta_{2}}^{\gamma \pi} \in \mathcal{B}(\mathcal{K}), \quad \text { for all } \eta_{1}, \eta_{2} \in \in_{\varphi}(\pi, \mathcal{H}) ; \\
& \left(\omega_{\tilde{\xi}_{1}, \xi_{2}} \gamma^{\star} \pi \operatorname{id}\right)(T)=\left(\lambda_{\tilde{\xi}_{1}}^{\gamma \pi}\right)^{*} T \lambda_{\tilde{\xi}_{2}}^{\gamma \pi} \in \mathcal{B}(\mathcal{H}), \quad \text { for all } \xi_{1}, \xi_{2} \in(\mathcal{K}, \gamma)_{\varphi} .
\end{aligned}
$$

If $x \in M_{\gamma^{\star} \pi} P$, then for all $\omega \in \mathcal{B}(\mathcal{H})_{*}\left(\right.$ resp. $\left.\omega \in \mathcal{B}(\mathcal{K})_{*}\right)$ we have $\left(\operatorname{id}_{\gamma \star \pi} \omega\right)(x) \in M$ (resp. $\left(\omega_{\gamma{ }^{\star} \pi}\right.$ id $)(x) \in P$ ). We refrain from writing the details but we can easily define the slice maps if $T$ takes its values in a different relative tensor product. Note that we can extend the notion of slice maps for normal linear forms to normal semi-finite weights.

fiber product over a finite-dimensional von neumann algebra. Now, let us assume that

$$
N:=\bigoplus_{1 \leqslant l \leqslant k} \mathrm{M}_{n_{l}}(\mathbb{C}) \quad \text { and } \quad \varphi:=\bigoplus_{1 \leqslant l \leqslant k} \operatorname{Tr}_{l}\left(F_{l}-\right),
$$

where $F_{l}$ is a positive invertible matrix of $\mathrm{M}_{n_{l}}(\mathbb{C})$ and $\operatorname{Tr}_{l}$ is the non-normalized trace on $\mathrm{M}_{n_{l}}(\mathbb{C})$. Denote by $\left(F_{l, i}\right)_{1 \leqslant i \leqslant n_{l}}$ the eigenvalues of $F_{l}$.

8.2.13 Proposition-Definition. - (\$7 [11]) The bounded linear map

$$
v_{\varphi}^{\gamma \pi}: \mathcal{K} \otimes \mathcal{H} \rightarrow \mathcal{K}_{\gamma} \otimes_{\varphi} \mathcal{H} ; \xi \otimes \eta \mapsto \xi_{\gamma} \otimes_{\varphi} \eta \quad \text { (or simply denoted by } v_{\gamma \pi} \text { ) }
$$

is a coisometry if, and only if, we have $\sum_{1 \leqslant i \leqslant n_{l}} F_{l, i}^{-1}=1$ for all $1 \leqslant l \leqslant k$.

In the following, we assume the above condition to be satisfied.

8.2.14 Proposition-Definition. - (\$7 [11]) Let us denote

$$
q_{\varphi}^{\gamma \pi}:=\left(v_{\varphi}^{\gamma \pi}\right)^{*} v_{\varphi}^{\gamma \pi} \quad\left(\text { or simply } q_{\gamma \pi}\right) .
$$

Then, $q_{\varphi}^{\gamma \pi}$ is a self-adjoint projection of $\mathcal{B}(\mathcal{K} \otimes \mathcal{H})$ such that

$$
q_{\varphi}^{\gamma \pi}=\sum_{1 \leqslant l \leqslant k} \sum_{1 \leqslant i, j \leqslant n_{l}} F_{l, i}^{-1 / 2} F_{l, j}^{-1 / 2} \gamma\left(e_{i j}^{(l) o}\right) \otimes \pi\left(e_{j i}^{(l)}\right),
$$

where, for all $1 \leqslant l \leqslant k,\left(e_{i j}^{(l)}\right)_{1 \leqslant i, j \leqslant n_{l}}$ is a system of matrix units (s.m.u.) diagonalizing $F_{l}$, i.e. $F_{l}=\sum_{1 \leqslant i \leqslant n_{l}} F_{l, i} e_{i i}^{(l)}$. Moreover, $M_{\gamma \star \pi} P \rightarrow q_{\varphi}^{\gamma \pi}(M \otimes P) q_{\varphi}^{\gamma \pi} ; x \mapsto\left(v_{\varphi}^{\gamma \pi}\right)^{*} x v_{\varphi}^{\gamma \pi}$ is a unital normal ${ }^{*}$-isomorphism.

Since $N$ is finite-dimensional, the inner product given by $\langle x, y\rangle:=\varphi\left(x^{*} y\right)$ for all $x, y \in N$ defines a structure of finite-dimensional Hilbert space on $N$. We have a (bounded) linear map $\mu_{\varphi}: N \otimes N \rightarrow N$ defined for all $x, y \in N$ by $\mu_{\varphi}(x \otimes y)=x y$, where $N \otimes N$ is endowed with its canonical structure of finite-dimensional Hilbert space.

8.2.15 Proposition-Definition. - For $i=1,2$, let $\pi_{i}: N \rightarrow \mathcal{B}\left(\mathcal{H}_{i}\right)$ be a unital normal *-representation of $N$ on a Hilbert space $\mathcal{H}_{i}$. Let us denote

$$
q_{\varphi}^{\pi_{1} \pi_{2}}:=\left(\pi_{1} \otimes \pi_{2}\right)\left(\mu_{\varphi}^{*}\left(1_{N}\right)\right) \in \mathcal{B}\left(\mathcal{H}_{1} \otimes \mathcal{H}_{2}\right) \quad\left(\text { or simply } q_{\pi_{1} \pi_{2}}\right) .
$$


We denote $q_{\varphi}^{\pi_{1}}:=q_{\varphi}^{\pi_{1} \pi_{1}}$ (or simply $q_{\pi_{1}}$ ) for short. Then, we have

$$
q_{\varphi}^{\pi_{1} \pi_{2}}=\sum_{1 \leqslant l \leqslant k} \sum_{1 \leqslant i, j \leqslant n_{l}} F_{l, j}^{-1} \pi_{1}\left(e_{i j}^{(l)}\right) \otimes \pi_{2}\left(e_{j i}^{(l)}\right),
$$

where, for all $1 \leqslant l \leqslant k,\left(e_{i j}^{(l)}\right)_{1 \leqslant i, j \leqslant n_{l}}$ is a s.m.u. diagonalizing $F_{l}$.

Proof. For $1 \leqslant l \leqslant k$, fix a s.u.m. $\left(e_{i j}^{(l)}\right)_{1 \leqslant i, j \leqslant n_{l}}$ of $\mathrm{M}_{n_{l}}(\mathbb{C})$ diagonalizing $F_{l}$. It suffices to prove that

$$
\mu_{\varphi}^{*}\left(1_{N}\right)=\sum_{1 \leqslant l \leqslant k} \sum_{1 \leqslant i, j \leqslant n_{l}} F_{l, j}^{-1} e_{i j}^{(l)} \otimes e_{j i}^{(l)}
$$

Since $1_{N}=\sum_{1 \leqslant l \leqslant k} \sum_{1 \leqslant i \leqslant n_{l}} e_{i i}^{(l)}$, it is enough to prove that

$$
\mu_{\varphi}^{*}\left(e_{r s}^{(l)}\right)=\sum_{1 \leqslant j \leqslant n_{l}} F_{l, j}^{-1} e_{r j}^{(l)} \otimes e_{j s}^{(l)}, \quad \text { for all } 1 \leqslant r, s \leqslant n_{l} .
$$

Let $\left(f_{i j}^{(l)}\right)$ be the family of $N$ given by $f_{i j}^{(l)}:=F_{l, j}^{-1 / 2} e_{i j}^{(l)}$ for all $1 \leqslant l \leqslant k$ and $1 \leqslant i, j \leqslant n_{l}$. It is clear that $\left(f_{i j}^{(l)}\right)$ is an orthonormal basis of $N$. We have

$$
\varphi\left(e_{s q}^{(l)}\right)=\operatorname{Tr}_{l}\left(F_{l} e_{s q}^{(l)}\right)=\sum_{i=1}^{n_{l}} F_{l, i} \operatorname{Tr}_{l}\left(e_{i i}^{(l)} e_{s q}^{(l)}\right)=F_{l, s} \operatorname{Tr}_{l}\left(e_{s q}^{(l)}\right)=F_{l, s} \delta_{q}^{s}
$$

We have

$$
\begin{aligned}
\mu_{\varphi}^{*}\left(e_{r s}^{(l)}\right) & =\sum_{l^{\prime}, l^{\prime \prime}=1}^{k} \sum_{i, j=1}^{n_{l^{\prime}}} \sum_{p, q=1}^{n_{l^{\prime \prime}}}\left\langle\mu_{\varphi}^{*}\left(e_{r s}^{(l)}\right), f_{i j}^{\left(l^{\prime}\right)} \otimes f_{p q}^{\left(l^{\prime \prime}\right)}\right\rangle f_{i j}^{\left(l^{\prime}\right)} \otimes f_{p q}^{\left(l^{\prime \prime}\right)} \\
& =\sum_{l^{\prime}, l^{\prime \prime}=1}^{k} \sum_{i, j=1}^{n_{l^{\prime}}} \sum_{p, q=1}^{n_{l^{\prime \prime}}} \delta_{l^{\prime \prime}}^{l^{\prime}} \delta_{p}^{j} F_{l^{\prime}, j}^{-1} F_{l^{\prime \prime}, q}^{-1}\left\langle e_{r s}^{(l)}, e_{i q}^{\left(l^{\prime}\right)}\right\rangle e_{i j}^{\left(l^{\prime}\right)} \otimes e_{p q}^{\left(l^{\prime \prime}\right)} \\
& =\sum_{l^{\prime}=1}^{k} \sum_{i, j, q=1}^{n_{l^{\prime}}} \delta_{l^{\prime}}^{l} \delta_{i}^{r} F_{l^{\prime}, j}^{-1} F_{l^{\prime}, q}^{-1} \varphi\left(e_{s q}^{(l)}\right) e_{i j}^{\left(l^{\prime}\right)} \otimes e_{j q}^{\left(l^{\prime}\right)} \\
& =\sum_{j, q=1}^{n_{l}} F_{l, j}^{-1} F_{l, q}^{-1} F_{l, s} \delta_{q}^{s} e_{r j}^{(l)} \otimes e_{j q}^{(l)} \\
& =\sum_{j=1}^{n_{l}} F_{l, j}^{-1} e_{r j}^{(l)} \otimes e_{j s}^{(l)} .
\end{aligned}
$$

8.2.16 Remarks. - $\quad$ 1. For $i=1,2$, let $\gamma_{i}: N^{\mathrm{o}} \rightarrow \mathcal{B}\left(\mathcal{K}_{i}\right)$ be a unital normal *-representation of $N^{\mathrm{o}}$ on a Hilbert space $\mathcal{K}_{i}$. In a similar way, we define $q_{\varphi^{\mathrm{o}}}^{\gamma_{1} \gamma_{2}} \in \mathcal{B}\left(\mathcal{K}_{1} \otimes \mathcal{K}_{2}\right)$ (or simply $\left.q_{\gamma_{1} \gamma_{2}}\right)$ such that

$$
q_{\varphi^{\mathrm{o}}}^{\gamma_{1} \gamma_{2}}=\sum_{1 \leqslant l \leqslant k} \sum_{1 \leqslant i, j \leqslant n_{l}} F_{l, j}^{-1} \gamma_{1}\left(e_{i j}^{(l) \mathrm{o}}\right) \otimes \gamma_{2}\left(e_{j i}^{(l) \mathrm{o}}\right),
$$

where, for all $1 \leqslant l \leqslant k,\left(e_{i j}^{(l)}\right)_{1 \leqslant i, j \leqslant n_{l}}$ is a s.m.u. diagonalizing $F_{l}$.

2. It should be noted that $q_{\varphi}^{\pi_{1} \pi_{2}}$ and $q_{\varphi^{\mathrm{O}}}^{\gamma_{1} \gamma_{2}}$ are self-adjoint but not idempotent in general. If $N$ is commutative (i.e. $N=\mathbb{C}^{k}$ ), then $q_{\varphi}^{\pi_{1} \pi_{2}}$ and $q_{\varphi^{\circ}}^{\gamma_{1} \gamma_{2}}$ are projections.

CASE OF THE NON-NORMALIZED MARKOV TRACE. In this paragraph, we take for $\varphi$ the non-normalized Markov trace on $N=\oplus_{1 \leqslant l \leqslant k} \mathrm{M}_{n_{l}}(\mathbb{C})$, i.e. $\epsilon=\oplus_{1 \leqslant l \leqslant k} n_{l} \cdot \operatorname{Tr}_{l}$. From now on, the operators $q_{\epsilon}^{\gamma \pi}, q_{\epsilon^{\mathrm{o}}}^{\pi \gamma} q_{\epsilon}^{\pi_{1} \pi_{2}}$ and $q_{\epsilon^{\mathrm{o}}}^{\gamma_{1} \gamma_{2}}$ will be simply denoted by $q_{\gamma \pi}, q_{\pi \gamma}, q_{\pi_{1} \pi_{2}}$ and $q_{\gamma_{1} \gamma_{2}}$. As a corollary of 8.2.14, we have:

8.2.17 Proposition. - For all s.u.m. $\left(e_{i j}^{(l)}\right)_{1 \leqslant l \leqslant k, 1 \leqslant i, j \leqslant n_{l}}$ of $N$, we have

$$
q_{\gamma \pi}=\sum_{1 \leqslant l \leqslant k} n_{l}^{-1} \sum_{1 \leqslant i, j \leqslant n_{l}} \gamma\left(e_{i j}^{(l) \mathrm{o}}\right) \otimes \pi\left(e_{j i}^{(l)}\right) \text { and } q_{\pi \gamma}=\sum_{1 \leqslant l \leqslant k} n_{l}^{-1} \sum_{1 \leqslant i, j \leqslant n_{l}} \pi\left(e_{i j}^{(l)}\right) \otimes \gamma\left(e_{j i}^{(l) \mathrm{o}}\right) \text {. }
$$


As a corollary of 8.2.15, we have:

8.2.18 Proposition. - For all s.u.m. $\left(e_{i j}^{(l)}\right)_{1 \leqslant l \leqslant k, 1 \leqslant i, j \leqslant n_{l}}$ of $N$, we have

$$
\begin{aligned}
q_{\pi_{1} \pi_{2}} & =\sum_{1 \leqslant l \leqslant k} n_{l}^{-1} \sum_{1 \leqslant i, j \leqslant n_{l}} \pi_{1}\left(e_{i j}^{(l)}\right) \otimes \pi_{2}\left(e_{j i}^{(l)}\right) \text { and } \\
q_{\gamma_{1} \gamma_{2}} & =\sum_{1 \leqslant l \leqslant k} n_{l}^{-1} \sum_{1 \leqslant i, j \leqslant n_{l}} \gamma_{1}\left(e_{i j}^{(l) \mathrm{o}}\right) \otimes \gamma_{2}\left(e_{j i}^{(l) \mathrm{o}}\right) .
\end{aligned}
$$

The following result is a slight generalization of 8.2 .17 to the setting of $\mathrm{C}^{*}$-algebras.

8.2.19 Proposition-Definition. - (2.6 [2]) Let $A, B$ be two $C^{*}$-algebras. We consider two non-degenerate ${ }^{*}$-homomorphisms $\gamma_{A}: N^{\mathrm{o}} \rightarrow \mathcal{M}(A)$ and $\pi_{B}: N \rightarrow \mathcal{M}(B)$. There exists a unique self-adjoint projection $q_{\gamma_{A} \pi_{B}} \in \mathcal{M}(A \otimes B)\left(\right.$ resp. $\left.q_{\pi_{B} \gamma_{A}} \in \mathcal{M}(B \otimes A)\right)$ such that

$$
\begin{aligned}
q_{\gamma_{A} \pi_{B}} & =\sum_{1 \leqslant l \leqslant k} n_{l}^{-1} \sum_{1 \leqslant i, j \leqslant n_{l}} \gamma_{A}\left(e_{i j}^{(l) \mathrm{o}}\right) \otimes \pi_{B}\left(e_{j i}^{(l)}\right) \\
\text { (resp. } q_{\pi_{B} \gamma_{A}} & \left.=\sum_{1 \leqslant l \leqslant k} n_{l}^{-1} \sum_{1 \leqslant i, j \leqslant n_{l}} \pi_{B}\left(e_{i j}^{(l)}\right) \otimes \gamma_{A}\left(e_{j i}^{(l) \mathrm{o}}\right)\right),
\end{aligned}
$$

for all s.u.m. $\left(e_{i j}^{(l)}\right)_{1 \leqslant l \leqslant k, 1 \leqslant i, j \leqslant n_{l}}$ of $N$.

Proof. The uniqueness of such a self-adjoint projection is straightforward. In virtue of the Gelfand-Naimark theorem, we can consider faithful non-degenerate ${ }^{*}$-homomorphisms $\theta_{A}: A \rightarrow \mathcal{B}(\mathcal{K})$ and $\theta_{B}: B \rightarrow \mathcal{B}(\mathcal{H})$. Let us denote $\gamma:=\theta_{A} \circ \gamma_{A}$ and $\pi:=\theta_{B} \circ \pi_{B}$. Then, $\gamma: N^{\mathrm{O}} \rightarrow \mathcal{B}(\mathcal{K})$ and $\pi: N \rightarrow \mathcal{B}(\mathcal{H})$ are normal unital *-representations. Let us fix an arbitrary s.u.m. $\left(e_{i j}^{(l)}\right)_{1 \leqslant i, j \leqslant n_{l}}$ for $\mathrm{M}_{n_{l}}(\mathbb{C})$ for each $1 \leqslant l \leqslant k$. We define a self-adjoint projection $q_{\gamma_{A} \pi_{B}} \in \mathcal{M}(A \otimes B)$ by setting:

$$
q_{\gamma_{A} \pi_{B}}:=\sum_{1 \leqslant l \leqslant k} n_{l}^{-1} \sum_{1 \leqslant i, j \leqslant n_{l}} \gamma_{A}\left(e_{i j}^{(l) o}\right) \otimes \pi_{B}\left(e_{j i}^{(l)}\right) .
$$

By 8.2.17, we have $q_{\gamma \pi}=\left(\theta_{A} \otimes \theta_{B}\right)\left(q_{\gamma_{A} \pi_{B}}\right)$. By using again 8.2.17 and the fact that $\theta_{A} \otimes \theta_{B}$ is faithful, we obtain that $q_{\gamma_{A} \pi_{B}}$ is independent of the chosen systems of matrix units. Moreover, the definition of $q_{\gamma_{A} \pi_{B}}$ shows that $q_{\gamma_{A} \pi_{B}}$ is also independent of the chosen faithful non-degenerate ${ }^{*}$-homomorphisms $\theta_{A}$ and $\theta_{B}$.

In a similar way, we have the following generalization of 8.2 .18 to the setting of $\mathrm{C}^{*}$-algebras.

8.2.20 Proposition. - For $i=1,2$, let $B_{i}$ (resp. $\left.A_{i}\right)$ be a $C^{*}$-algebra and $\pi_{i}: N \rightarrow \mathcal{M}\left(B_{i}\right)$ (resp. $\gamma_{i}: N^{\mathrm{o}} \rightarrow \mathcal{M}\left(A_{i}\right)$ ) a non-degenerate ${ }^{*}$-homomorphism. Then, there exists a unique $q_{\pi_{1} \pi_{2}} \in \mathcal{M}\left(B_{1} \otimes B_{2}\right)$ (resp. $q_{\gamma_{1} \gamma_{2}} \in \mathcal{M}\left(A_{1} \otimes A_{2}\right)$ ) such that

$$
\begin{aligned}
q_{\pi_{1} \pi_{2}} & =\sum_{1 \leqslant l \leqslant k} n_{l}^{-1} \sum_{1 \leqslant i, j \leqslant n_{l}} \pi_{1}\left(e_{i j}^{(l)}\right) \otimes \pi_{2}\left(e_{j i}^{(l)}\right) \\
\text { (resp. } q_{\gamma_{1} \gamma_{2}} & \left.=\sum_{1 \leqslant l \leqslant k} n_{l}^{-1} \sum_{1 \leqslant i, j \leqslant n_{l}} \gamma_{1}\left(e_{i j}^{(l) \mathrm{o}}\right) \otimes \gamma_{2}\left(e_{j i}^{(l) \mathrm{o}}\right)\right),
\end{aligned}
$$

for all s.u.m. $\left(e_{i j}^{(l)}\right)_{1 \leqslant l \leqslant k, 1 \leqslant i, j \leqslant n_{l}}$ of $N$.

In the following, we adopt a multi-index notation to simplify formulas and computations.

8.2.21 Notations. - $\quad$ 1. Consider the index sets $\mathscr{I}:=\left\{(l, i, j) ; 1 \leqslant l \leqslant k, 1 \leqslant i, j \leqslant n_{l}\right\}$ and $\mathscr{I}_{0}:=\mathscr{I} \sqcup\{\varnothing\}$.

2. For $I=(l, i, j) \in \mathscr{I}$, we denote $\bar{I}:=(l, j, i) \in \mathscr{I}$. Denote also $\bar{\varnothing}:=\varnothing$. The map $\mathscr{I}_{0} \rightarrow \mathscr{I}_{0} ; I \mapsto \bar{I}$ is involutive. 
3. A pair of indices $(I, J) \in \mathscr{I} \times \mathscr{I}$ is said to be composable if we have $I=(l, i, m)$ and $J=(l, m, j)$ for some indices $1 \leqslant l \leqslant k$ and $1 \leqslant i, m, j \leqslant n_{l}$. In this case, we denote $I J:=(l, i, j) \in \mathscr{I}$. We also denote $I J=\varnothing$ if $I$ and $J$ are not composable, $I=\varnothing$ or $J=\varnothing$. This defines a map $\mathscr{I}_{0} \times \mathscr{I}_{0} \rightarrow \mathscr{I}_{0} ;(I, J) \mapsto I J$. It is clear that $\overline{I J}=\bar{J} \bar{I}$ for all $I, J \in \mathscr{I}_{0}$.

Let us fix a s.u.m. $\left(e_{i j}^{(l)}\right)_{1 \leqslant l \leqslant k, 1 \leqslant i, j \leqslant n_{l}}$ of $N$.

8.2.22 Notations. - $\quad$ 1. Denote by $\varepsilon_{I}:=e_{i j}^{(l)}$ for $I=(l, i, j) \in \mathscr{I}$ and $\varepsilon_{\varnothing}:=0$. Denote by $e_{I}:=\pi\left(\varepsilon_{I}\right)$ and $f_{I}:=\gamma\left(\varepsilon_{I}^{\mathrm{o}}\right)$ for $I \in \mathscr{I}_{0}$. Denote by $n_{I}:=n_{l}$ for $I=(l, i, j) \in \mathscr{I}$ and $n_{\varnothing}:=1$. Notice that we have $n_{\bar{I}}=n_{I}$ for all $I \in \mathscr{I}_{0}$.

2. Since $\left(\varepsilon_{I}\right)_{I \in \mathscr{I}}$ is a basis of $N$, for $x \in N$ we denote $x=\sum_{I \in \mathscr{I}} x_{I} \cdot \varepsilon_{I}$, with $x_{I} \in \mathbb{C}$ for $I \in \mathscr{I}$. Note that $x^{*}=\sum_{I \in \mathscr{I}} \overline{x_{\bar{I}}} \cdot \varepsilon_{I}$.

8.2.23 Remarks. - $\quad$ 1. For all $I, J \in \mathscr{I}_{0}$, we have $\varepsilon_{I}^{*}=\varepsilon_{\bar{I}}$ and $\varepsilon_{I} \varepsilon_{J}=\varepsilon_{I J}$. For all $I, J \in \mathscr{I}_{0}$, we have:

$$
e_{I}^{*}=e_{\bar{I}}, \quad e_{I} e_{J}=e_{I J} ; \quad f_{I}^{*}=f_{\bar{I}}, \quad f_{I} f_{J}=f_{J I} .
$$

2. We have $q_{\gamma \pi}=\sum_{I \in \mathscr{I}} n_{I}^{-1} f_{I} \otimes e_{\bar{I}}, q_{\pi \gamma}=\sum_{I \in \mathscr{I}} n_{I}^{-1} e_{I} \otimes f_{\bar{I}}, q_{\pi}=\sum_{I \in \mathscr{I}} e_{I} \otimes e_{\bar{I}}$ and $q_{\gamma}=\sum_{I \in \mathscr{I}} f_{I} \otimes f_{\bar{I}}$.

\subsection{Unitary equivalence of Hilbert $C^{*}$-modules}

In the following, we recall the notion of morphism between Hilbert modules over possibly different $C^{*}$-algebras.

8.3.1 Definition. - Let $A$ and $B$ be two $C^{*}$-algebras and $\phi: A \rightarrow B$ a ${ }^{*}$-homomorphism. Let $\mathscr{E}$ and $\mathscr{F}$ be two Hilbert $C^{*}$-modules over $A$ and $B$ respectively. A $\phi$-compatible operator from $\mathscr{E}$ to $\mathscr{F}$ is a linear map $\Phi: \mathscr{E} \rightarrow \mathscr{F}$ such that:

(i) for all $\xi \in \mathscr{E}$ and $a \in A, \Phi(\xi a)=\Phi(\xi) \phi(a)$;

(ii) for all $\xi, \eta \in \mathscr{E},\langle\Phi \xi, \Phi \eta\rangle=\phi(\langle\xi, \eta\rangle)$.

Furthermore, if $\phi$ is a *-isomorphism and $\Phi$ is surjective, we say that $\Phi$ is $\phi$-compatible unitary operator (or a unitary equivalence over $\phi$ ) from $\mathscr{E}$ onto $\mathscr{F}$.

8.3.2 REMARKs. - $\quad$ 1. It follows from (ii) that $\Phi: \mathscr{E} \rightarrow \mathscr{F}$ is bounded and even isometric if $\phi$ is faithful. Indeed, we have $\|\langle\Phi \xi, \Phi \eta\rangle\|=\|\phi(\langle\xi, \eta\rangle)\|=\|\langle\xi, \eta\rangle\|$ for all $\xi, \eta \in \mathscr{E}$. Then, for all $\xi \in \mathscr{E}$ we have $\|\Phi \xi\|^{2}=\|\langle\Phi \xi, \Phi \xi\rangle\|=\|\langle\xi, \xi\rangle\|=\|\xi\|^{2}$. In particular, if $\phi$ is a *-isomorphism and $\Phi$ is a $\phi$-compatible unitary operator, then $\Phi$ is bijective and the inverse map $\Phi^{-1}: \mathscr{F} \rightarrow \mathscr{E}$ is a $\phi^{-1}$-compatible unitary operator.

2. It is clear that $\mathrm{id}_{\mathscr{E}}$ is a $\mathrm{id}_{A}$-compatible unitary operator. Let $A, B$ and $C$ be $C^{*}$-algebras and $\mathscr{E}, \mathscr{F}$ and $\mathscr{G}$ be Hilbert modules over $A, B$ and $C$ respectively. Let $\phi: A \rightarrow B$ and $\psi: B \rightarrow C$ be ${ }^{*}$-homomorphisms (resp. ${ }^{*}$-isomorphisms). If $\Phi: \mathscr{E} \rightarrow \mathscr{F}$ is a $\phi$-compatible operator (resp. unitary operator) and $\Psi: \mathscr{F} \rightarrow \mathscr{G}$ a $\psi$-compatible operator (resp. unitary operator), then $\Psi \circ \Phi: \mathscr{E} \rightarrow \mathscr{G}$ is a $\psi \circ \phi$-compatible operator (resp. unitary operator).

3. Let $\Phi: \mathscr{E} \rightarrow \mathscr{F}$ be a unitary equivalence over a given ${ }^{*}$-isomorphism $\phi$. If $T \in \mathcal{L}(\mathscr{E})$, then the map $\Phi \circ T \circ \Phi^{-1}: \mathscr{F} \rightarrow \mathscr{F}$ is an adjointable operator whose adjoint operator is $\Phi^{-1} \circ T^{*} \circ \Phi$. We define a *-isomorphism $\mathcal{L}(\mathscr{E}) \rightarrow \mathcal{L}(\mathscr{F}) ; T \mapsto \Phi \circ T \circ \Phi^{-1}$. Note that $\Phi \circ \theta_{\xi, \eta} \circ \Phi^{-1}=\theta_{\Phi \xi, \Phi \eta}$ for all $\xi, \eta \in \mathscr{E}$. In particular, for all $k \in \mathcal{K}(\mathscr{E})$ we have $\Phi \circ k \circ \Phi^{-1} \in \mathcal{K}(\mathscr{F})$. More precisely, the map $\mathcal{K}(\mathscr{E}) \rightarrow \mathcal{K}(\mathscr{F}) ; k \mapsto \Phi \circ k \circ \Phi^{-1}$ is a *-isomorphism.

The notion of unitary equivalence defines an equivalence relation on the class consisting of all Hilbert $C^{*}$-modules (cf. 8.3.2 1, 2). Actually, this notion of morphism between Hilbert modules over possibly different $C^{*}$-algebra can be understood in terms of unitary adjointable operator between two Hilbert modules over the same $C^{*}$-algebra. 
8.3.3 Proposition. - Let $A$ and $B$ be two $C^{*}$-algebras and $\phi: A \rightarrow B a^{*}$-isomorphism. Let $\mathscr{E}$ and $\mathscr{F}$ be two Hilbert $C^{*}$-modules over $A$ and $B$ respectively.

1. If $\Phi: \mathscr{E} \rightarrow \mathscr{F}$ is a surjective $\phi$-compatible unitary operator, then there exists a unique unitary adjointable operator $U \in \mathcal{L}\left(\mathscr{E} \otimes_{\phi} B, \mathscr{F}\right)$ such that $U\left(\xi \otimes_{\phi} b\right)=\Phi(\xi) b$, for all $\xi \in \mathscr{E}$ and $b \in B$.

2. Conversely, if $U \in \mathcal{L}\left(\mathscr{E} \otimes_{\phi} B, \mathscr{F}\right)$ is a unitary, then there exists a unique $\phi$-compatible unitary operator $\Phi: \mathscr{E} \rightarrow \mathscr{F}$ such that $\Phi(\xi) b=U\left(\xi \otimes_{\phi} b\right)$ for all $\xi \in \mathscr{E}$ and $b \in B$.

As an application of the above proposition, we can the state the following result.

8.3.4 Proposition-Definition. - Let $A_{1}, B_{1}, A_{2}$ and $B_{2}$ be $C^{*}$-algebras, $\phi_{1}: A_{1} \rightarrow B_{1}$ and $\phi_{2}: A_{2} \rightarrow B_{2}{ }^{*}$-isomorphisms. Let $\mathscr{E}_{1}, \mathscr{F}_{1}, \mathscr{E}_{2}$ and $\mathscr{F}_{2}$ be Hilbert $C^{*}$-modules over $A_{1}, B_{1}, A_{2}$ and $B_{2}$ respectively. Let $\Phi_{1}: \mathscr{E}_{1} \rightarrow \mathscr{F}_{1}$ and $\Phi_{2}: \mathscr{E}_{2} \rightarrow \mathscr{F}_{2}$ be unitary equivalences over $\phi_{1}$ and $\phi_{2}$ respectively. Then, the linear map $\mathscr{E}_{1} \odot \mathscr{E}_{2} \rightarrow \mathscr{F}_{1} \otimes \mathscr{F}_{2} ; \xi_{1} \otimes \xi_{2} \mapsto \Phi_{1}\left(\xi_{1}\right) \otimes \Phi_{2}\left(\xi_{2}\right)$ extends to a bounded linear map $\Phi_{1} \otimes \Phi_{2}: \mathscr{E}_{1} \otimes \mathscr{E}_{2} \rightarrow \mathscr{F}_{1} \otimes \mathscr{F}_{2}$. Moreover, $\Phi_{1} \otimes \Phi_{2}$ is a $\phi_{1} \otimes \phi_{2}$-compatible unitary operator.

The notion of unitary equivalence can also be understood in terms of isomorphism between the associated linking $\mathrm{C}^{*}$-algebras.

8.3.5 Proposition. - Let $A$ and $B$ be two $C^{*}$-algebras and $\phi: A \rightarrow B a^{*}$-isomorphism. Let \& and $\mathscr{F}$ be two Hilbert $C^{*}$-modules over $A$ and $B$ respectively.

1. If $\Phi: \mathscr{E} \rightarrow \mathscr{F}$ is a $\phi$-compatible unitary operator, then there exists a unique *homomorphism $f: \mathcal{K}(\mathscr{E} \oplus A) \rightarrow \mathcal{K}(\mathscr{F} \oplus B)$ such that $f \circ \iota_{\mathscr{E}}=\iota_{\mathscr{F}} \circ \Phi$ and $f \circ \iota_{A}=\iota_{B} \circ \phi$. Moreover, $f$ is $a^{*}$-isomorphism.

2. Conversely, let $f: \mathcal{K}(\mathscr{E} \oplus A) \rightarrow \mathcal{K}(\mathscr{F} \oplus B)$ be a ${ }^{*}$-isomorphism such that $f \circ \iota_{A}=\iota_{B} \circ \phi$. Then, there exists a unique map $\Phi: \mathscr{E} \rightarrow \mathscr{F}$ such that $f \circ \iota_{\mathscr{E}}=\iota_{\mathscr{F}} \circ \Phi$. Moreover, $\Phi$ is a $\phi$-compatible unitary operator.

Proof. 1. The ${ }^{*}$-homomorphism $f: \mathcal{K}(\mathscr{E} \oplus A) \rightarrow \mathcal{K}(\mathscr{F} \oplus B)$ is defined by (cf. 8.3.2 3$)$ :

$$
f\left(\begin{array}{cc}
k & \xi \\
\eta^{*} & a
\end{array}\right):=\left(\begin{array}{cc}
\Phi \circ k \circ \Phi^{-1} & \Phi \xi \\
(\Phi \eta)^{*} & \phi(a)
\end{array}\right), \quad \text { for all } k \in \mathcal{K}(\mathscr{E}), \xi, \eta \in \mathscr{E} \text { and } a \in A \text {. }
$$

2. This is a straightforward consequence of 2.3 .41 .

8.3.6 Notation. - Let $A, B$ be $C^{*}$-algebras and $\mathscr{E}$ and $\mathscr{F}$ be two Hilbert $C^{*}$-modules over $A$ and $B$ respectively. Let $\phi: A \rightarrow B$ be a ${ }^{*}$-isomorphism and $\Phi: \mathscr{E} \rightarrow \mathscr{F}$ a $\phi$-compatible unitary operator. If $T \in \mathcal{L}(A, \mathscr{E})$, we define the map $\widetilde{\Phi}(T):=\Phi \circ T \circ \phi^{-1}: B \rightarrow \mathscr{F}$. By a straightforward computation, we show that $\widetilde{\Phi}(T) \in \mathcal{L}(B, \mathscr{F})$ whose adjoint operator is $\widetilde{\Phi}(T)^{*}=\phi \circ T^{*} \circ \Phi^{-1}$. We have a bounded linear map $\widetilde{\Phi}: \mathcal{L}(A, \mathscr{E}) \rightarrow \mathcal{L}(B, \mathscr{F})$, which is an extension of $\Phi$ up to the canonical injections $\mathscr{E} \rightarrow \mathcal{L}(A, \mathscr{E})$ and $\mathscr{F} \rightarrow \mathcal{L}(B, \mathscr{F})$.

\section{REFERENCES}

[1] S. BAAJ, Repésentation régulière du groupe quantique des déplacements de Woronowicz, Astérisque 232 (1995), 11-49.

[2] S. BAAj et J. Crespo, Équivalence monoïdale de groupes quantiques et $K$-théorie bivariante, Bull. Soc. Math. France 145 (4) (2017), 711-802.

[3] S. BaAj et G. SKandalis, $C^{*}$-algèbres de Hopf et théorie de Kasparov équivariante, K-theory 2 (1989), 683-721.

[4] S. BaAJ et G. SKandalis, Unitaires multiplicatifs et dualité pour les produits croisés de C*-algèbres, Ann. Sci. Éc. Norm. Supér. 4 e série, 26 (4) (1993), 425-488.

[5] S. BaAJ, G. Skandalis and S. Vaes, Non-semi-regular quantum groups coming from number theory, Comm. Math. Phys. 235 (1) (2003), 139-167. 
[6] J. Bichon, A. De Rijdt and S. Vaes, Ergodic coactions with large multiplicity and monoidal equivalence of quantum groups, Comm. Math. Phys. 262 (2006), 703-728.

[7] A. Connes, On the spatial theory of von Neumann algebras, J. Funct. Anal. 35 (1980), 153-164.

[8] A. Connes, Noncommutative Geometry, Academic Press, San Diego, CA, 1994.

[9] J. CRespo, Monoidal equivalence of locally compact quantum groups and application to bivariant K-theory, Ph.D. thesis, Université Blaise Pascal (2015).

[10] J. CRespo, Measured quantum groupoid on a finite basis and equivariant Kasparov theory, preprint: arXiv:1706.08516 [math.OA].

[11] K. De Commer, Monoidal equivalence for locally compact quantum groups, preprint: arXiv:math.OA/0804.2405v2.

[12] K. De Commer, Galois coactions for algebraic and locally compact quantum groups, Ph.D. thesis, Leuven, Katholieke Universiteit Leuven (2009).

[13] K. De Commer, Galois coactions and cocycle twisting for locally compact quantum groups, J. Operator theory 66 (1) (2011), 59-106.

[14] K. De Commer, A. Freslon and M. Yamashita, CCAP for Universal Discrete Quantum Groups (with an appendix by S. Vaes), Comm. Math. Phys. 331 (2) (2014), 677-701.

[15] A. De Rijdt and N. VAnder Vennet, Actions of monoidally equivalent compact quantum groups and applications to probabilistic boundaries, Ann. Inst. Fourier 60 (1) (2010), 169-216.

[16] M. Enock, Quantum groupoids of compact type, J. Inst. Math. Jussieu 4 (2005), $29-133$.

[17] M. Enock, Measured Quantum Groupoids in Action, Mém. Soc. Math. Fr. 114 (2008), 1-150.

[18] G. G. Kasparov, Hilbert $C^{*}$-modules: theorems of Stinespring and Voiculescu, J. Operator Theory 4 (1) (1980), 133-150.

[19] J. Kustermans and S. VAes, Locally compact quantum groups in the von Neumann algebraic setting, Math. Scand. 92 (1) (2003), 68-92.

[20] F. Lesieur, Measured Quantum Groupoids, Mém. Soc. Math. Fr. 109 (2007), 1-117.

[21] S. Neshveyev and L. Tuset, Deformation of $C^{*}$-algebras by cocycles on locally compact quantum groups, Adv. Math. 254 (2014), 454-496.

[22] M. A. RIEFFEL, Induced representations of $C^{*}$-algebras, Adv. Math. 13 (2) (1974), 176257.

[23] T. Timmermann, Pseudo-multiplicative unitaries and pseudo-Kac systems on C*modules, 05/2005, Dissertation, Preprint des SFB 478 Münster (394).

[24] T. Timmermann, Coactions of Hopf C*-bimodules, J. Operator Theory 68 (1) (2012), 19-66.

[25] S. VAES, The unitary implementation of a locally compact quantum group action, $J$. Funct. Anal. 180 (2001), 426-480.

[26] S. Vaes and N. VAnder Vennet, Identification of the Poisson and Martin boundaries of orthogonal discrete quantum groups, J. Inst. Math. Jussieu 7 (2008), 391-412.

[27] J.-M. VAllin, Unitaire pseudo-multiplicatif associé à un groupoïde. Applications à la moyennabilité, J. Operator Theory 44 (2) (2000), 347-368.

[28] R. Vergnioux and C. Voigt, The K-theory of free quantum groups, Math. Ann. 357 (1) (2013), 355-400. 
[29] C. Voigt, The Baum-Connes conjecture for free orthogonal quantum groups, Adv. Math. 227 (5) (2011), 1873-1913.

VRIJE Universiteit Brussel, Vakgroep Wiskunde, Pleinlaan 2, B-1050 Brussels (Belgium).

E-mail addresses: jonathan.crespo@wanadoo.fr, jonathan.crespo@vub.ac.be

Supported by the FWO grant G.0251.15N 
$\operatorname{Alg}_{\mathcal{G}}$, category of $\mathcal{G}$-C ${ }^{*}$-algebras, 22

$A \rtimes \mathcal{G}$, crossed product, 23

$B \rtimes \widehat{\mathcal{G}}$, crossed product, 23

$\mathcal{C}(-), 6$

$C_{M}, 63$

$\Delta_{i j}^{k}, 15$

$\delta_{i j}^{k}, 16$

$\delta_{A}^{2}$, iterated coaction map, 21

$D$, bidual $\mathcal{G}$-C $\mathrm{C}^{*}$-algebra, 24

$\delta_{A_{j}, 25}^{k}$

$\delta_{A_{1}, 26}^{(2)}$

$\delta_{\mathscr{E}_{j}, 42}^{k}$

$\delta_{\mathscr{E}_{1}}^{(2)}, 44$

$\left(\varepsilon_{1}, \varepsilon_{2}\right)$, standard basis of $\mathbb{C}^{2}, 14$

$\mathcal{E}_{A, L}, 22$

$\mathcal{E}_{B, \lambda}, 23$

$\mathcal{E}_{A, R}, 24$

$\varepsilon_{I}, 70$

$e_{I}, f_{I}, 70$

$\mathcal{G}_{\mathrm{G}_{1}, \mathrm{G}_{2}}$, colinking measured quantum groupoid, 16

$\mathscr{H}_{i j}, 15$

$\iota_{A}, l_{\mathscr{E}}, \iota_{\mathscr{E}}, \iota_{\mathcal{K}(\mathscr{E})}$, canonical morphisms, 7 $\iota_{i j}^{k}, 16$

$\operatorname{Ind}_{\mathrm{G}_{1}}^{\mathrm{G}_{2}}\left(A_{1}\right)$, induced $\mathrm{C}^{*}$-algebra, 26

$\operatorname{Ind}_{\mathrm{G}_{1}}^{\mathrm{G}_{2}}\left(\mathscr{E}_{1}\right)$, induced Hilbert module, 44

$\mathscr{I}$, multi-index set, 69

$j_{D}, 24$

$\mathcal{K}, \mathrm{C}^{*}$-algebra of compact operators on the G.N.S. space $\mathrm{L}^{2}(\mathcal{G}), 17$

$\mathcal{K}_{\pi}, \mathcal{K}_{\gamma}, 6_{5}$

$L, R, \rho, \lambda$, canonical representations of $S$ and $\widehat{S}, 13$

$\mathrm{M}_{n_{l}}(\mathbb{C})$, square matrices of order $n_{l}$ with entries in $\mathbb{C}, 2$

$\widetilde{\mathcal{M}}(\mathscr{E} \otimes B)$, relative multiplier module, 8 $M_{i j}, 15$

$\mathfrak{N}_{\varphi}, \mathfrak{M}_{\varphi}^{+}, 63$

$N_{+}^{\text {ext }}$, extended positive cone, 63
$\mathfrak{N}_{T}, \mathfrak{M}_{T}^{+}, \mathfrak{M}_{T}, 64$

$\bar{\omega}, 62$

$\omega_{\tilde{\zeta}, \eta}, 62$

$p_{i j}, 14$

$\varphi_{i j}, \psi_{i j}, 15$

$\pi_{L, 22}$

$\pi, \widehat{\theta}, 22$

$\hat{\pi}_{\lambda}, 23$

$\widehat{\pi}, \theta, 23$

$\pi_{R, 24}$

$\pi_{j}^{k}, \pi_{A, j}^{k}, 25$

$\pi_{j}, 26$

$\tilde{\pi}_{j, 27}$

$\Pi_{j}^{k}, 40,53$

$\Pi_{j}, 51$

$\widetilde{\Pi}_{j}, 53$

$\varphi^{\mathrm{O}}$, opposite weight, 63

$\varphi^{\mathrm{c}}$, commutant weight, 65

$q_{j}, q_{A, j}, 25$

$q_{I}, 28$

$q_{\mathscr{8}, j}, 40$

$q_{\gamma \pi}, q_{\pi \gamma}, 67$

$q_{\pi_{1} \pi_{2}}, q_{\pi_{1}}, 67$

$R_{\mathcal{G}}$, unitary coinverse, 10

$R_{\xi}^{\pi}, L_{\eta}^{\gamma}, 65$

$S, \widehat{S}$, weak Hopf $C^{*}$-algebras, 13

$S_{i j}, 16$

$\sigma_{\gamma \pi} / \varsigma_{\gamma \pi}$, relative flip

map $/{ }^{*}$-homomorphism, 66

$\operatorname{Tr}_{l}$, non-normalized Markov trace on

$$
\mathrm{M}_{n_{l}}(\mathbb{C}), 11
$$

$T_{\tilde{\zeta}}, 31$

$U, 10$

$\widehat{\mathcal{V}}, \mathcal{V}, \widetilde{\mathcal{V}}, 11$

$V, W, \widetilde{V}$, multiplicative partial isometries,

$V_{j l}^{i}, W_{i k}^{j}, \stackrel{12}{\widetilde{V}_{k i}^{j}}, 15$

$\mathcal{V}, 24$

$\mathcal{V}_{j}^{k}, 41,52$

$v_{\gamma \pi}$, canonical coisometry, 67

$W_{\mathcal{G}}$, pseudo-multiplicative unitary, 10

$\mathcal{Z}(-)$, center, 10 